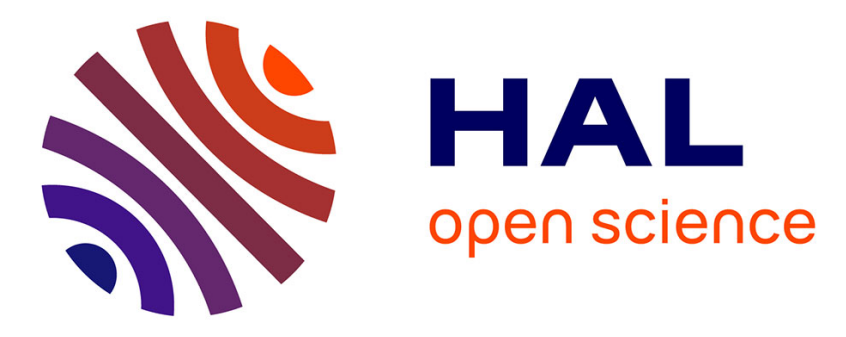

\title{
Divergent synthesis of $5^{6}, 7^{6}$-difluorinated dihydroxanthene-hemicyanine fused near-infrared fluorophores
}

Shasha Zheng, Gu Lingyue, Michelle Jui Hsien Ong, Denis Jacquemin, Anthony Romieu, Jean-Alexandre Richard, Rajavel Srinivasan

\section{To cite this version:}

Shasha Zheng, Gu Lingyue, Michelle Jui Hsien Ong, Denis Jacquemin, Anthony Romieu, et al.. Divergent synthesis of 5,7 '-difluorinated dihydroxanthene-hemicyanine fused near-infrared fluorophores. Organic \& Biomolecular Chemistry, 2019, 17 (17), pp.4291-4300. 10.1039/c9ob00568d . hal-02140853

\section{HAL Id: hal-02140853 \\ https://hal.science/hal-02140853}

Submitted on 30 Dec 2021

HAL is a multi-disciplinary open access archive for the deposit and dissemination of scientific research documents, whether they are published or not. The documents may come from teaching and research institutions in France or abroad, or from public or private research centers.
L'archive ouverte pluridisciplinaire HAL, est destinée au dépôt et à la diffusion de documents scientifiques de niveau recherche, publiés ou non, émanant des établissements d'enseignement et de recherche français ou étrangers, des laboratoires publics ou privés. 


\section{Divergent Synthesis of 5',7'-Difluorinated Dihydroxanthene-Hemicyanine Fused Near- Infrared Fluorophores}

Shasha Zheng, ${ }^{a}$ Gu Lingyue, ${ }^{a}$ Michelle Jui Hsien Ong, ${ }^{b}$ Denis Jacquemin, ${ }^{* c}$ Anthony Romieu, ${ }^{* d, e}$ Jean-Alexandre Richard ${ }^{* b}$ and Rajavel Srinivasan*a

We describe an expedient access to a $5^{\prime}, 6^{\prime}, 7^{\prime}$-trifluoro dihydroxanthene-hemicyanine fused scaffold in 2 steps and $54 \%$ overall yield from the corresponding salicylic aldehyde. A 6'-regioselective nucleophilic aromatic substitution ( $\mathrm{S}_{N} \mathrm{Ar}$ ) reaction with a wide range of nitrogen, sulfur or selenium nucleophiles then gives access to 16 near-infrared (NIR) fluorophores emitting in the $710-750 \mathrm{~nm}$ range. We also report the experimental and theoretical photophysical investigations of these unique optical agents that include the first series of $6^{\prime}$-heavy atom substituted dihydroxanthenes, extending the pool of polyfluorinated markers for biomedical and material applications.

\section{Introduction}

In their quest to discover useful small molecules and organic materials, chemists resort to a variety of strategies to optimize their structure and function. One of them is the replacement of $\mathrm{C}-\mathrm{H}$ bonds by $\mathrm{C}-\mathrm{F}$ linkages which often provide specific and valuable properties (e.g., small size of fluorine, high polarization and strength of the $\mathrm{C}-\mathrm{F}$ bond, etc.... $)^{1}$ beneficial for the development of pharmaceuticals, ${ }^{2}$ agrochemicals, ${ }^{3}$ materials, ${ }^{4}$ and optical devices. ${ }^{5}$ The fluorine atom finds use in many applications like polytetrafluoroethylene polymers (i.e., Teflon) ${ }^{6}$ and is currently estimated to be present in $25 \%$ of pharmaceuticals ${ }^{7}$ and agrochemicals. ${ }^{8}$ Fluorine is also useful for diagnostics with ${ }^{18} \mathrm{~F}$ positron emission tomography $(\mathrm{PET})^{9}$ and magnetic resonance imaging (MRI), ${ }^{10}{ }^{19} \mathrm{~F}$ nuclear magnetic resonance (NMR) analysis ${ }^{11}$ or the synthesis and purification of organic molecules. ${ }^{12}$ Because of its high sensitivity and attractive safety profile, fluorescence optical imaging is an indispensable technique in medical diagnosis and the

\footnotetext{
S. Zheng, G. Lingyue, Prof. R. Srinivasan

School of Pharmaceutical Science and Technology (SPST), Tianjin University,

Building 24, 92 Weijin Road, Nankai District, Tianjin, 300072 P. R. China

E-mail: rajavels@tju.edu.cn

b.Dr. J.-A. Richard

Organic Chemistry

Institute of Chemical and Engineering Sciences (ICES), Agency for Science,

Technology and Research (A*STAR)

8 Biomedical Grove, Neuros, \#07-01, Singapore 138665

E-mail: jean alexandre@ices.a-star.edu.sg

Prof. D. Jacquemin

Chimie Et Interdisciplinarité, Synthèse, Analyse, Modélisation (CEISAM), UMR CNRS no. 6230, BP 92208, Université de Nantes, 2, Rue de la Houssinière, 4432 Nantes Cedex 3, France

E-mail: Denis.Jacquemin@univ-nantes.fr

d.Prof. A. Romieu

ICMUB, UMR 6302, CNRS, Univ. Bourgogne Franche-Comte

9, Avenue Alain Savary, 21078 Dijon cedex, France

E-mail: anthony.romieu@u-bourgogne.fr

e. Prof. A. Romieu

Institut Universitaire de France

1, Rue Descartes, Bâtiment MONGE, 75231 Paris, France
}

A) Coumarin, rhodamine and BODIPY polyfluorinated fluorophores

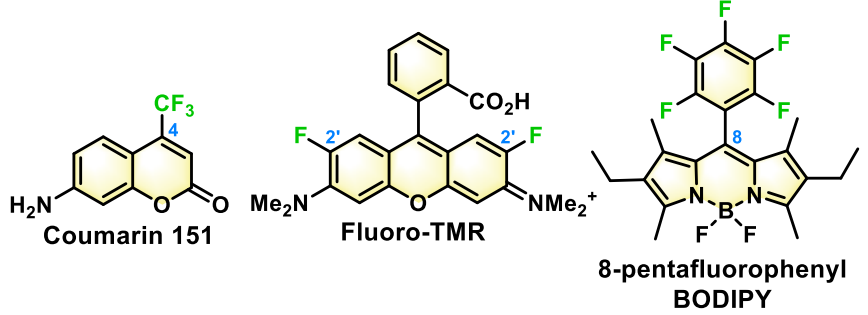

B) $S_{N} A r$ strategy for the synthesis of functionalized polyfluorinated BODIPY
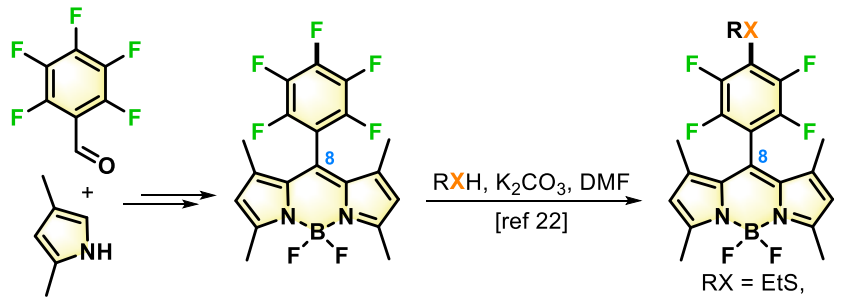

$\mathrm{BuNH}$ or $\mathrm{Et}_{2} \mathrm{~N}$

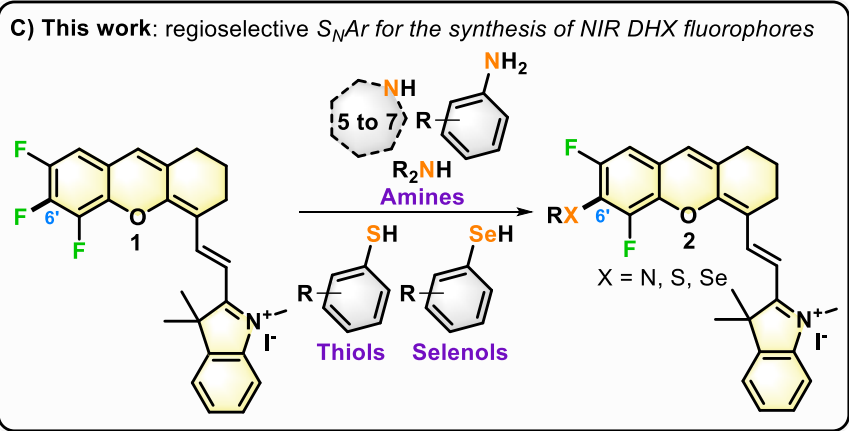

Scheme 1 A) Representative polyfluorinated fluorophores; B) Nucleophilic aromatic substitution ( $\mathrm{S}_{\mathrm{N}} \mathrm{Ar}$ ) of a pentafluorinated BODIPY fluorophore; $\mathrm{C}$ ) Proposed regioselective, late-stage $\mathrm{S}_{N} \mathrm{Ar}$ for the synthesis of $5^{\prime}, 7^{\prime}$-difluoro $\mathrm{DHX}$ hemicyanine fused dyes 2. 


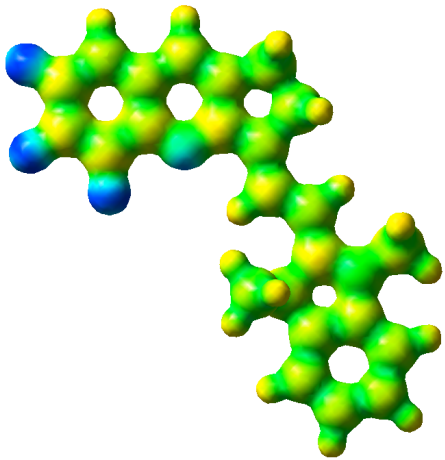

Fig. 1 Representation of the DFT electrostatic potential of the model compound 1. Blue/yellow regions correspond the most negatively/positively charged regions, see computational details.

introduction of fluorine atoms within the core structure of organic-based fluorophores is an appealing concept for improving the performances of these small-molecule markers. ${ }^{13}$ For instance, the attachment of polyfluorinated tags on rhodamines, ${ }^{14}$ oxazine and squaraine dyes ${ }^{15}$ has been performed and polyfluorinated cyanines have been reported to form J-aggregates in fluorous solvents. ${ }^{16}$ Coumarins, xanthene, ${ }^{17}$ BODIPY ${ }^{18}$ and cyanine dyes ${ }^{19}, 20$ bearing fluorine atoms have also been prepared and feature improved optical properties compared to their non-fluorinated counterparts (Scheme 1A). [ $\left.{ }^{18} \mathrm{~F}\right]$-radiolabelled far-red and NIR-emitting bimodal cyanine dyes have also been described. ${ }^{21}$ In this context, the efficiency of the nucleophilic aromatic substitution $\left(\mathrm{S}_{N} \mathrm{Ar}\right)$ reactions reported by the $\mathrm{McClenaghan}^{22}$ and Wiehe ${ }^{23}$ groups where a nucleophile regioselectively attacks the mesopentafluorinated ring of a BODIPY fluorophore attracted our attention (Scheme 1B). In this case, the spectral signatures of the penta- and tetrafluorinated fluorophores were highly similar and we wondered if we could perform a similar sitespecific transformation on a different fluorophore where the replacement of fluorine atom by a heteroatom would positively influence its photophysical properties. In particular, we aimed at developing polyfluorinated fluorophores on a scaffold emitting in the near-infrared (NIR) because of the advantages long wavelengths provide when used in biological systems. ${ }^{24}$ To the best of our knowledge, such fluorinated NIR fluorophores have seldom been described ${ }^{25}$ and we decided to explore the reactivity of the dihydroxanthene (DHX) skeleton ${ }^{26}$ since its structural modularity and attractive emission profile makes it an interesting alternative to other more conventional and popular cyanine dyes. ${ }^{27}$ Leveraging on a methodology we previously developed to synthesize $\mathrm{DHX}$ cores, $^{28}$ we aimed at preparing a polyfluorinated precursor to perform a late-stage, regioselective diversification giving access to a wide range of polyfluorinated analogues. We therefore prepared the trifluorinated dihydroxanthene precursor 1 and demonstrated that a wide range of nitrogen, sulfur and selenium nucleophiles could regioselectively displace the $6^{\prime}-\mathrm{F}$ position to lead to unique 6-heterosubstituted NIR 5',7'-difluorinated DHXhemicyanine fused dyes 2 (Scheme $1 \mathrm{C}$ ). We evaluated the spectral properties of these long-wavelength fluorinated xanthene-like fluorophores either in simulated physiological conditions or in organic media and performed Time-Dependent Density Functional Theory (TD-DFT) computations to get insights into their unusual electronic absorption properties.

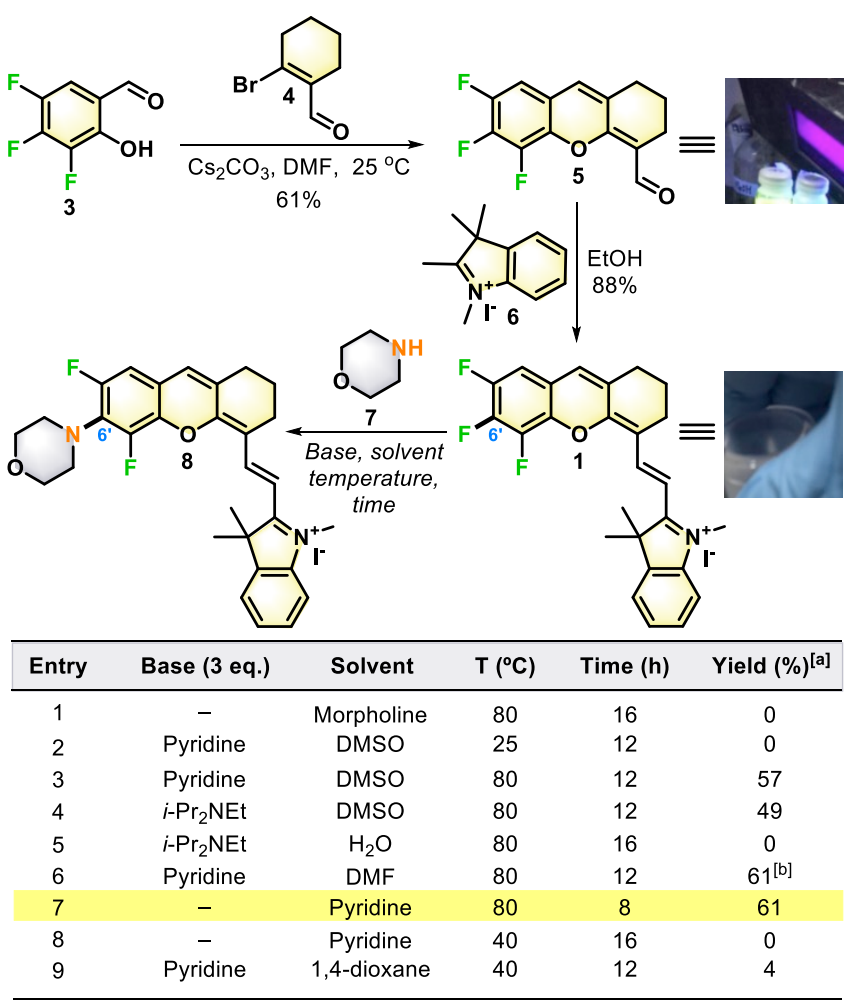

[a] Yield recorded after purification by silica-gel chromatography. [b] Incomplete conversion.

Scheme 2 Synthesis of the $5^{\prime}, 6^{\prime}, 7^{\prime}$-trifluoro DHX-hemicyanine fused dye precursor 1 and optimization of the late-stage $S_{N} A r$ reaction.

\section{Results and discussion}

Late-stage $S_{N} A r$ for the synthesis of a library of $5^{\prime}, 7^{\prime}$-difluoro dihydroxanthene-hemicyanine fused fluorophores

Before addressing the synthetic aspect of the study, we wondered if computation could provide us any intelligence regarding the feasibility of such a $6^{\prime}$-regioselective $S_{N} A r$ reaction. We first investigated the electrostatic potential of the molecule but it was inconclusive because all C-F functions showed very similar potential. We therefore turned to conceptual DFT to determine which one of the $5^{\prime}-, 6^{\prime}$ - or $7^{\prime}$ carbon atoms was the most electrophilic by comparing the natural charges determined on the DHX molecule $\mathbf{1}$ in its neutral and charged counterparts. The change of charge is twice larger on the $6^{\prime}$ carbon $(-0.039 e)$ as compared to $7^{\prime}$ and $5^{\prime}$ which undergo smaller variations of $-0.017 e$ and $-0.010 e$, respectively, confirming that the $6^{\prime}$ position is the most reactive toward nucleophiles (Fig. 1). Consequently, we performed the synthesis of the bright yellow trifluorinated precursor $\mathbf{5}$ which we prepared in one step $\left(\mathrm{Cs}_{2} \mathrm{CO}_{3}, \mathrm{DMF}\right)$ and $61 \%$ yield from the reaction between 3,4,5-trifluoro-2-hydroxybenzaldehyde $\mathbf{3}$ (prepared from the Duff formylation of 2,3,4-trifluorophenol, see $\mathrm{ESI}^{\dagger}$ ) and 6-bromocyclohex-1-ene-1-carbaldehyde 4. The formation of the deep blue-colored dye 1 was then achieved in $88 \%$ yield in the presence of the Fisher's base 1,2,3,3tetramethyl-3H-indol-1-ium iodide $\mathbf{6}$ in EtOH. Having 


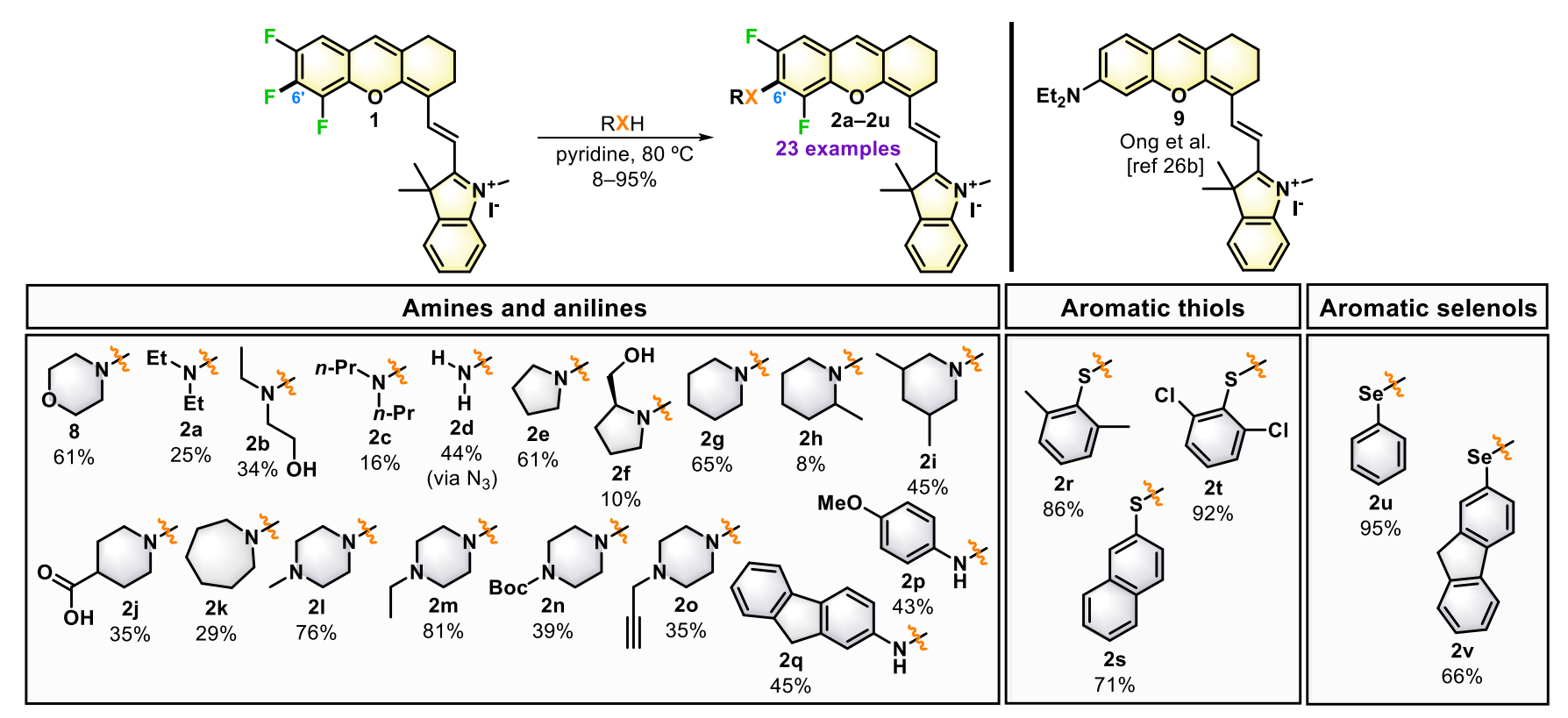

Scheme 3 Scope of the late-stage $S_{N} A r$ for the synthesis of 5',7'-difluoro DHX-hemicyanine fused dyes $2 \mathbf{a}-2 \mathbf{v}$ and $\mathbf{8}$.

successfully synthesised the advanced intermediate $\mathbf{1}$ in gram scale, we assessed our hypothesis regarding the late-stage, 6'regioselective $S_{N} A r$ reaction. Using morpholine (5 equiv.) as model nitrogen nucleophile, we detected no product when we carried out the reaction in this cyclic secondary amine as solvent or in DMSO at room temperature in the presence of pyridine (Scheme 2, entries 1-2). However, we were delighted to observe the product formation in $57 \%$ yield when we performed the same reaction at $80^{\circ} \mathrm{C}$ for $12 \mathrm{~h}$ (Scheme 2, entry 3). The reaction not only cleanly led to mono-substituted product and was selective for the anticipated 6 '-position. Encouraged by this result, we screened a panel of solvents, bases and temperatures to optimize the transformation. Replacing pyridine by Hünig's base resulted in a slight drop of product formation in DMSO (49\% yield) and no product in water (Scheme 2, entries 4-5). Using pyridine in DMF provided a good $61 \%$ yield but under these conditions, the isolation of the pure product was challenging because of incomplete conversion (Scheme 2, entry 6). We also obtained the best yield of $61 \%$ when we carried out the reaction in a sealed tube with neat pyridine at $80^{\circ} \mathrm{C}$ for $8 \mathrm{~h}$ (Scheme 2, entry 7). In contrast, no product formed at $40{ }^{\circ} \mathrm{C}$, a trend confirmed using 1,4-dioxane as solvent which showed the importance of temperature for the efficiency of the reaction (Scheme 2, entry 8-9). With this set of optimised reaction conditions in hand, we then tested an array of nucleophiles including aliphatic and aromatic amines, morpholine, piperazines, 5-, 6-, and 7-membered nitrogen containing saturated heterocycles, aryl thiols and selenols (Scheme 3 ). While the reaction with bis-alkylated amines provided fluorophores $\mathbf{2 a - 2 c}$ in moderate yields (16-34\% yield), the use of $\mathrm{NaN}_{3}$ as a nucleophile provided the resulting azido product which undergoes formal reduction during purification to furnish the aniline derivative $\mathbf{2} \mathbf{d}$ ( $44 \%$ yield). The $\mathrm{S}_{N} \mathrm{Ar}$ reaction was also successful with pyrrolidine $\mathbf{2 e}$ and piperidine $\mathbf{2 g}$ (61\%, and $65 \%$, respectively) but the yield dropped for substituted pyrrolidine $\mathbf{2 f}$ (10\%), piperidines $\mathbf{2} \mathbf{h}-\mathbf{2} \mathbf{j}$ ( $8 \%, 45 \%$ and $35 \%$, respectively) as well as for the 7-membered ring azepane $\mathbf{2 k}$ (29\% yield), showing a sensitivity of the reaction for the steric hindrance of the amine nucleophile. $\mathrm{N}$-Alkylated piperazines $\mathbf{2 l - 2 0}$ were also tolerated (35-81\% yield) as well as less nucleophilic aromatic amines 4-methoxyaniline $\mathbf{2 p}$ and 2 -aminofluorene $\mathbf{2 q}(\mathbf{4} 3 \%$ and $45 \%$, respectively). The influence of the nucleophilicity was investigated with the use of thiophenols and aromatic selenols which showed increased yields $(95 \%$ and $66 \%$ yield for $\mathbf{2 u}$ and 2v) even for sterically hindered o-disubstituted 2,6dimethylthiophenol $2 r$ ( $86 \%$ yield) and 2,6-dichlorothiophenol 2t $(92 \%$ yield). We underline that some of the substrates obtained are bifunctional (i.e., $\mathbf{2 b}, \mathbf{2 f}, \mathbf{2} \mathbf{j}, \mathbf{2 n}$ and $\mathbf{2 0}$ ) and could be post-synthetically derivatised for further functionalization (e.g., introduction of polar groups for water solubility) or conjugation to (bio)molecular partners.

\section{Photophysical properties of the $5^{\prime}, 7^{\prime}$-difluoro dihydroxanthene- hemicyanine fused fluorophores}

We evaluated the photophysical properties of our library of 23 difluoro-DHX-hemicyanine fused dyes $\mathbf{2 a - 2 v}$ and $\mathbf{8}$ in different media including phosphate buffered saline (PBS) with $5 \%(\mathrm{w} / \mathrm{v})$ bovine serum albumin (BSA) as simulated body fluid (only for the less hydrophobic derivatives), ${ }^{29} \mathrm{EtOH}$, and $\mathrm{CHCl}_{3}$ (Table 1, Fig. 2 for Abs/Ex/Em spectra of $\mathbf{2 a}$ and $\mathbf{2 e}$ in $\mathrm{CHCl}_{3}$ and in PBS + $5 \%$ BSA, see ESIt for Abs/Ex/Em spectra of $\mathbf{2 d}, \mathbf{2 g}, \mathbf{2 k}$ and $\mathbf{8}$ ). All 6 '-substituted DHX derivatives display an intense absorption band in the far-red and/or NIR region. However, compared to non-fluorinated parent compounds previously studied by us, ${ }^{28 b}$ this main band, assigned to the $\mathrm{S}_{0}-\mathrm{S}_{1}$ electronic transition, is much broader and structureless with no clear vibronic structure observable. For instance, $N, N$-diethyl derivatives $2 a$ and $\mathbf{9}$ exhibit full-width half maximum (FWHM), $\Delta \lambda_{1 / 2} \max$ in the range 139-167 $\mathrm{nm}$ and 36-51 $\mathrm{nm}$ respectively, depending on the solvent used for spectral measurements (Fig. 2). As confirmed 
Table 1 Photophysical properties of 5',7'-difluoro DHX-hemicyanine fused dyes $1,2 \mathrm{a}-\mathbf{v}$ and 8 at $25^{\circ} \mathrm{C}$. For structures, see Scheme 3 .

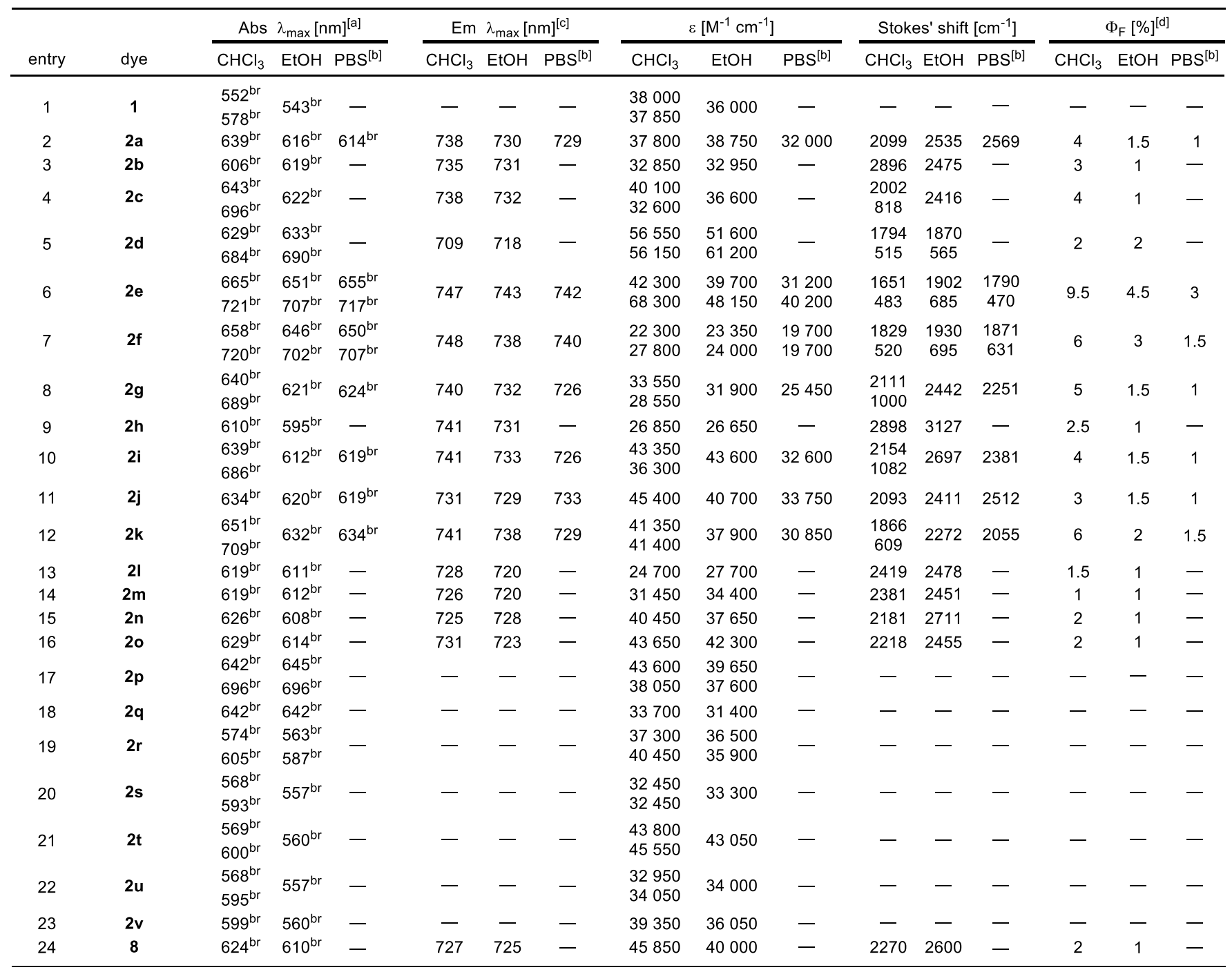

${ }^{a}$ Assigned to $S_{0}-S_{1}$ transition (see Fig. 2 and the Supporting Information for selected examples of Abs spectra). ${ }^{b}$ PBS buffer containing $5 \%$ BSA. ${ }^{c}$ Excitation at $615 \mathrm{~nm} .{ }^{d}$ Determined at $25^{\circ} \mathrm{C}$ by using sulfoindocyanine dye Cy $5.0\left(\Phi_{\mathrm{F}}=20 \%\right.$ in PBS, $\left.\lambda_{\mathrm{ex}}=615 \mathrm{~nm}\right)$ as standard. ${ }^{40}$

by TD-DFT calculations (vide infra), the presence of two orthofluorine atoms within $\mathbf{2 a}$ could prevent the presence in solution of a single absorbing species in which the electron-donating $\mathrm{NEt}_{2}$ group and DHX chromophore are co-planar and fullconjugated. The sole difluoro-DHX-hemicyanine fused dye for which the behavior of the absorption spectrum is relatively similar to that of the corresponding non-fluorinated analog is the $\mathbf{N}$-pyrrolidinyl derivative $\mathbf{2 e}$. Thus, the size and geometry of its azolidine ring are probably optimal to avoid adverse steric interferences with fluorine atoms which are at the origin of multiple conformers (rotation around the $\mathrm{C} 6{ }^{\prime}-\mathrm{N}$ bond) in solution, and hence broader spectra. As anticipated, all 6'-N,Ndialkylamino DHX-hemicyanine hybrids $\mathbf{2 a - 2 0}$ and 8 show an emission peak in the NIR region, with the maximum in the 709$747 \mathrm{~nm}$ range depending on the solvent used and the nature of nitrogen substituents. The good match between the absorption and excitation spectra of $\mathbf{2 e}$ confirms the presence of a single absorbing/emissive species in all tested media. As expected, this trend is not observed for the fluorinated fluorophores existing as a mixture of multiple conformers, such as $\mathbf{2 a}$. The lower values of fluorescence quantum yield than those obtained with non-fluorinated fluorophores (e.g., $\Phi_{\mathrm{F}}$ of $\mathbf{9}$ is $30 \%$ and $10 \%$ in $\mathrm{CHCl}_{3}$ and in PBS $+5 \%$ BSA respectively) ${ }^{28 b}$ could be due to the intramolecular charge transfer from electron-rich heteroatom to DHX core and are in line with the prominence of non-radiative decay processes intertwined with the conformational equilibrium previously put forward. Complete extinction of the NIR emission is obtained through the introduction of an $\mathrm{N}$-aryl substituent or aryl thiol/selenol moieties (compounds $\mathbf{2 p - 2 v}$, see ESIt for selected examples of Abs spectra). For these latter hetero-functionalizations, the lack of a substituent with a good electron-donating ability and/or the spin-orbit (heavy-atom) effects promoting triplet state formation relative to fluorescence, are reasonable assumptions to explain this quenching. ${ }^{30}$ These readily accessible longwavelength chromophores would therefore be interesting candidates to be used as dark quencher molecules for the 

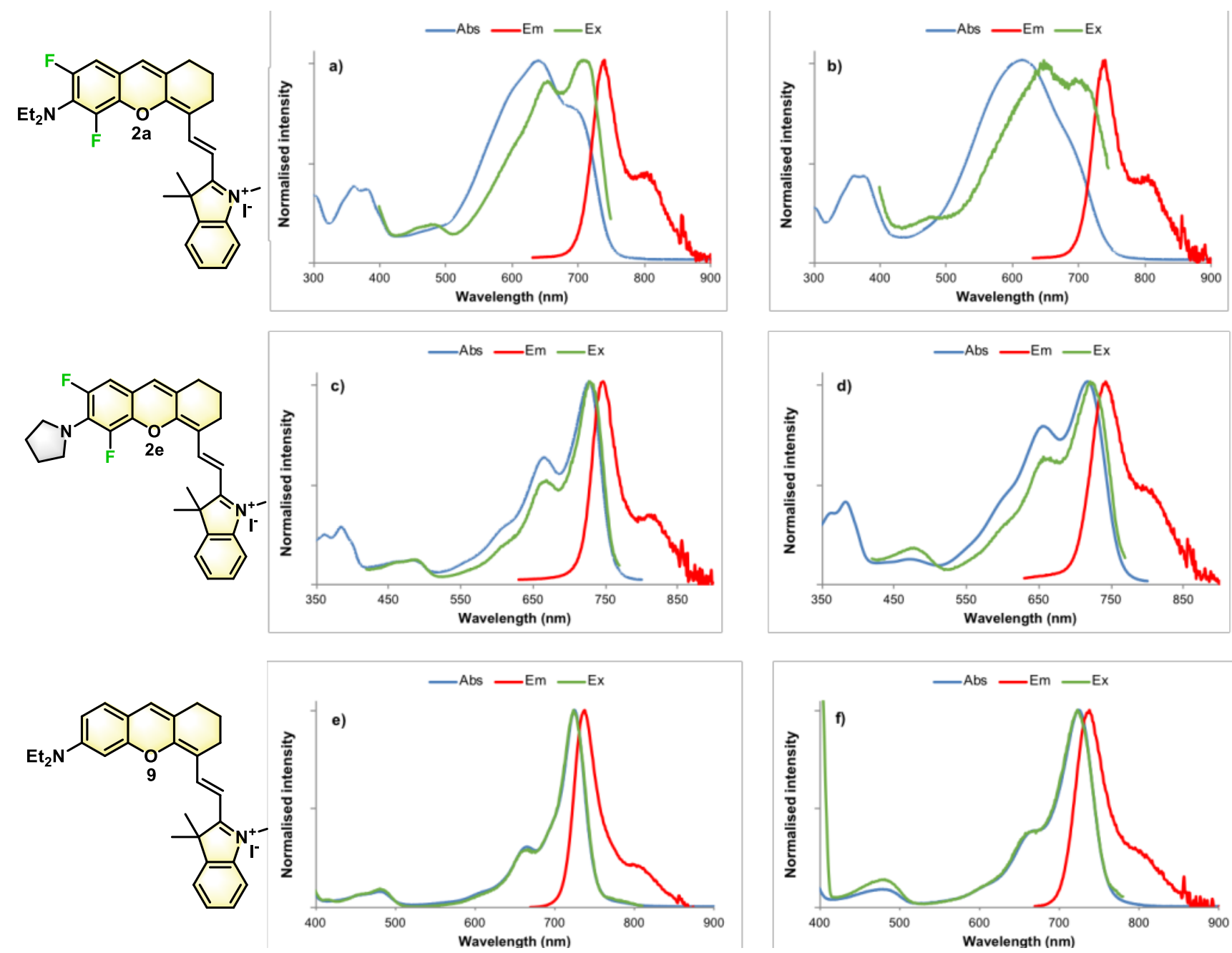

Fig. 2 Normalised absorption, emission (excitation at $615 \mathrm{~nm}$ for a-d and $650 \mathrm{~nm}$ for e-f) and excitation (emission at $760 \mathrm{~nm}$ for a-d, $830 \mathrm{~nm}$ for e or $800 \mathrm{~nm}$ for f) spectra of fluorinated $\mathrm{N}, \mathrm{N}$-dialkylamino-DHX NIR fluorophores $2 \mathrm{a}\left(\mathrm{a}\right.$ in $\mathrm{CHCl}_{3}$ and b in PBS $+5 \% \mathrm{BSA}$ ), $2 \mathrm{e}(\mathrm{c}$ in $\mathrm{CHCl} 3$ and d in PBS $+5 \% \mathrm{BSA}$ ), and nonfluorinated parent NIR fluorophore 9 (e in $\mathrm{CHCl}_{3}$ and $\mathrm{f}$ in PBS $\left.+5 \% \mathrm{BSA}\right)$. For the excitation spectrum of 9 in PBS $+5 \%$ BSA, a peak at $400 \mathrm{~nm}\left(\lambda_{\mathrm{ex}} / 2\right)$ assigned to Rayleigh scattering is observed. See Experimental section for details about these measurements.

construction of NIR fluorescent probes based on Förster resonance energy transfer (FRET) mechanism. ${ }^{31}$

\section{TD-DFT computation for selected 5',7'-difluoro dihydroxanthene-hemicyanine fused fluorophores}

The density difference plots for two representative examples, 8 and $\mathbf{2 e}$, are displayed in Fig. 3. Consistently with our previous work, ${ }^{32}$ we observe a clear $\pi-\pi^{*}$ character for the electronic transition with changes of opposite sign on the vicinal carbon atoms of the hemicyanine moiety. As expected, the pushing $\mathrm{N}, \mathrm{N}$-dialkylamino group plays the role of a donor (nitrogen in blue in Fig. 3). We have then used theory to investigate the impact of the electron-donating group on the photophysical properties, and in particular, the broadness of the observed electronic absorption spectra (Table 2, vide supra). For 8, we could locate two non-equivalent true minima, differing by the relative twist of the donor group with respect to the plane of the core of the dye, on both the ground ( $\left.S_{0}\right)$ and excited (S1) state potential energy surfaces. For the $S_{0}$ state, the dihedral angle between the two subsystems is vastly different $\left(24^{\circ}\right.$ and $\left.67^{\circ}\right)$, but the computed free energy difference between the two structures is negligible $(<1 \mathrm{kcal} / \mathrm{mol})$, implying a fast conversion between the two (i.e., a series of intermediate states). As expected, the computed absorption energies vastly differ for these two compounds (by ca. $0.3 \mathrm{eV}$ at the CC2 level). In contrast, when going to the $S_{1}$ state, the most planar molecule (now with a $32^{\circ}$ dihedral) becomes significantly more stable (by $3.2 \mathrm{kcal} / \mathrm{mol}$ ). This theoretical result is in line with the experimental trends that show a broad absorption, but a standard emission for $\mathbf{8}$. The computed 0-0 energies for the two species are 1.667 and $1.898 \mathrm{eV}$, logically bracketing the experimental value. For $\mathbf{2 d}$, we found only one conformation for the $\mathrm{NH}_{2}$ group with a twist of the $\mathrm{NH}$ bond, with respect to the dye's plane, of $20^{\circ}$ and $8^{\circ}$ in $S_{0}$ and $S_{1}$, respectively. There is therefore limited flexibility and the absorption spectrum is tight. The theoretical 0-0 energy is $1.803 \mathrm{eV}$, very close to the experimental value of $1.783 \mathrm{eV}$. For $\mathbf{2 e}$, we started with the pyrrolidinyl group either perfectly in the plane of the dye or twisted, but both optimizations led to the in-plane structure in 

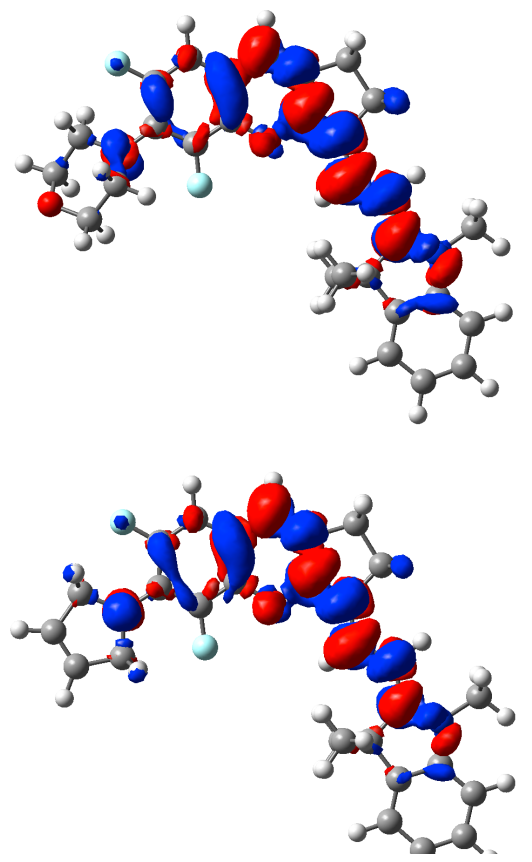

Fig. 3 Density difference plot $\left[\Delta \rho=\rho\left(S_{1}\right)-\rho\left(S_{0}\right)\right]$ obtained for 8 (top) and $\mathbf{2 e}$ (bottom). The blue and red regions indicate loss and gain of electronic density upon absorption, respectively. Contour threshold: $8 \times 10^{-4}$ au.

both the $\mathrm{S}_{0}$ and $\mathrm{S}_{1}$ states. The computed 0-0 energy (1.655 eV) is strongly red-shifted compared $\mathbf{2 d}$, which is perfectly consistent with the experimental trend (0-0 of $1.690 \mathrm{eV})$. For $\mathbf{2 g}$, starting with a planar and perpendicular conformation of the side group, we obtained two slightly different minima, both showing similar twisting angles of $25^{\circ}\left(\mathrm{S}_{0}\right)$ and ca. $35^{\circ}\left(\mathrm{S}_{1}\right)$. For both conformers, the relative free energies are almost equal (differences $<1 \mathrm{kcal} / \mathrm{mol}$ ) and the same holds for the $0-0$ energies: 1.622 and $1.645 \mathrm{eV}$. These values do not agree so well with the experimental value of $1.722 \mathrm{eV}$. For 2a, we could find several orientations of the $\mathrm{NEt}_{2}$ donor groups all inside a 1 $\mathrm{kcal} / \mathrm{mol}$ window (relative $\mathrm{S}_{0}$ free energies), with however significantly different absorption maxima, explaining the broad spectra (see Table 2). In the excited-state, different twisting angles are still obtained depending on the conformers but they present more alike fluorescence energies. The 0-0 energies obtained are very similar to $\mathbf{2 g}$, which is consistent with experiment, though the 0-0 energies are undershot, as in the previous case. Finally, for $\mathbf{2 k}$ we could also find two different conformers but as for $\mathbf{2} \mathbf{g}$ they do present very similar spectroscopic signatures, which is consistent with the relatively small width at half maximum observed experimentally for $\mathbf{2 k}$.

\section{Conclusions}

In summary, we have developed a divergent access to 6'heterosubstituted $5^{\prime}, 7^{\prime}$-difluoro dihydroxanthene-hemicyanine fused dyes through a 6 -selective and effective $S_{N} A r$ reaction. Conceptual DFT allowed us to predict the regioselectivity of the displacement of an aryl fluoride moiety which exclusively led to the $6^{\prime}$-substitution. The transformation was successful using a wide range of amino, thio, and seleno nucleophiles and provided either fluorophores emitting in the $710-750 \mathrm{~nm}$ range
Table 2. Comparison of the experimental and theoretical optical properties of the 5',7'-difluoro DHX-hemicyanine fused dyes. In several cases, different conformers (conf.) are obtained. The theoretical results have been obtained at the CC2//CAM-B3LYP level of theory (see Experimental Section for details).

\begin{tabular}{|c|c|c|c|c|c|c|c|}
\hline dye & conf. & $\begin{array}{c}\lambda_{\text {abs }}[\text { the }] \\
{[\mathrm{nm}]}\end{array}$ & $\begin{array}{c}\lambda_{\text {abs }}[\exp ] \\
{[\mathrm{nm}]}\end{array}$ & $\begin{array}{c}\lambda_{\mathrm{fl}}[\text { the }] \\
{[\mathrm{nm}]}\end{array}$ & $\begin{array}{c}\lambda_{\mathrm{fl}}[\exp ] \\
{[\mathrm{nm}]}\end{array}$ & $\begin{array}{c}0-0[\text { the }] \\
{[\mathrm{eV}]}\end{array}$ & $\begin{array}{c}0-0[\exp ] \\
{[\mathrm{eV}]}\end{array}$ \\
\hline \multirow[t]{3}{*}{$2 a$} & a & 673 & 639 (br) & 775 & 738 & 1.640 & 1.727 \\
\hline & b & 643 & & 783 & & 1.629 & \\
\hline & c & 671 & & 777 & & 1.632 & \\
\hline $2 d$ & - & 600 & 684 & 713 & 709 & 1.803 & 1.783 \\
\hline $2 f$ & - & 671 & 721 & 770 & 747 & 1.655 & 1.671 \\
\hline \multirow[t]{2}{*}{$2 g$} & $a$ & 653 & 689 & 784 & 740 & 1.622 & 1.722 \\
\hline & b & 649 & & 782 & & 1.645 & \\
\hline \multirow[t]{2}{*}{$2 k$} & a & 679 & 709 & 789 & 741 & 1.613 & 1.701 \\
\hline & b & 679 & & 782 & & 1.633 & 1.633 \\
\hline 8 & Planar & 630 & $62 \Delta(\mathrm{hr})$ & $77 n$ & 727 & 1 אศ7 & 1771 \\
\hline
\end{tabular}

in aqueous and organic media or non-emissive NIR chromophores potentially usable as NIR fluorescence quenchers. The various broadness of the absorption and emission spectra have been correlated to the presence (or absence) of several conformers of similar energies. As a general rule, bis-fluorination of DHX scaffold led to a drop of fluorescence efficiency compared to the parent non-fluorinated fluorophores, even if a "hit" photoactive compound bearing a pyrrolidinyl as $N, N$-dialkylamino electron-donating group and exhibiting a good fluorescence quantum yield of $10 \%$ in $\mathrm{CHCl}_{3}$, was identified. We hope that the attractive features of this $\mathrm{S}_{N} \mathrm{Ar}$ based functionalization procedure (i.e., simplicity and ease of implementation) will encourage its further use in the conversion of DHX-hemicyanine hybrid scaffolds to reactionbased small-molecule fluorescent probes and promote their use in other biomedical applications. ${ }^{27,} 33$

\section{Experimental}

See ESIt for the details about sections "General", "Instruments and methods", and all experimental and spectral data associated with synthesised compounds.

\section{Synthesis}

$5^{\prime}, 6^{\prime}, 7^{\prime}$-Trifluoro-2,3-dihydro-1H-xanthene-4-carbaldehyde (5) To 2-hydroxyl-3,4,5-trifluoro-benzaldehyde 3 (528 mg, $3 \mathrm{mmol}, 1$ equiv) and $\mathrm{Cs}_{2} \mathrm{CO}_{3}$ (978 mg, $3 \mathrm{mmol}, 1$ equiv) in DMF (15 mL) was added 6bromocyclohex-1-ene-1-carbaldehyde (4) (2.26 g, $12 \mathrm{mmol}, 4$ equiv) and the mixture was stirred at $25^{\circ} \mathrm{C}$ for $56 \mathrm{~h}$. The reaction mixture was filtered and concentrated in vacuo. Purification by flash-column chromatography on silica gel ( $10 \%$ EtOAc in petroleum ether) afforded $5^{\prime}, 6^{\prime}, 7^{\prime}$-trifluoro-2,3-dihydro-1H-xanthene-4-carbaldehyde

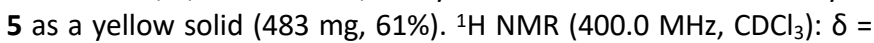
$10.35(\mathrm{~s}, 1 \mathrm{H}), 6.78(\mathrm{~m}, 1 \mathrm{H}), 6.53(\mathrm{~s}, 1 \mathrm{H}), 2.59(\mathrm{t}, J=5.8 \mathrm{~Hz}, 2 \mathrm{H}), 2.45$ $(\mathrm{t}, J=6.1 \mathrm{~Hz}, 2 \mathrm{H}), 1.73(\mathrm{~m}, 2 \mathrm{H}) \mathrm{ppm} ;{ }^{19} \mathrm{~F} \mathrm{NMR}\left(376.5 \mathrm{MHz}, \mathrm{CDCl}_{3}\right): \delta=$ $-140.7(\mathrm{~m}, 1 \mathrm{~F}),-155.2(\mathrm{~m}, 1 \mathrm{~F}),-155.8(\mathrm{~m}, 1 \mathrm{~F}) \mathrm{ppm} ;{ }^{13} \mathrm{C}$ NMR $(100.0$ 
$\left.\mathrm{MHz}, \mathrm{CDCl}_{3}\right): \delta=188.1,157.9,146.9\left(\mathrm{ddd}, J_{\mathrm{C}-\mathrm{F}}=2.1 \mathrm{~Hz}, J_{\mathrm{C}-\mathrm{F}}=11.1 \mathrm{~Hz}\right.$, $\left.{ }^{1} J_{\mathrm{C}-\mathrm{F}}=246.6 \mathrm{~Hz}\right), 140.6\left(\mathrm{ddd}, J_{\mathrm{C}-\mathrm{F}}=12.1 \mathrm{~Hz}, J_{\mathrm{C}-\mathrm{F}}=16.9 \mathrm{~Hz},{ }^{1} J_{\mathrm{C}-\mathrm{F}}=254.2\right.$ $\mathrm{Hz}), 139.8\left(\mathrm{ddd}, J_{\mathrm{C}-\mathrm{F}}=4.0 \mathrm{~Hz}, J_{\mathrm{C}-\mathrm{F}}=12.5 \mathrm{~Hz},{ }^{1} J_{\mathrm{C}-\mathrm{F}}=253.8 \mathrm{~Hz}\right), 137.4(\mathrm{~m})$, $131.5\left(\mathrm{~d}, J_{\mathrm{C}-\mathrm{F}}=2.9 \mathrm{~Hz}\right), 123.6\left(\mathrm{dd}, J_{\mathrm{C}-\mathrm{F}}=3.1 \mathrm{~Hz}, J_{\mathrm{C}-F}=5.7 \mathrm{~Hz}\right), 117.1(\mathrm{~m})$, $115.2\left(d, J_{C-F}=0.9 \mathrm{~Hz}\right), 107.8\left(\mathrm{dd}, J_{\mathrm{C}-\mathrm{F}}=3.7 \mathrm{~Hz}, J_{\mathrm{C}-\mathrm{F}}=19.6 \mathrm{~Hz}\right), 30.1$, 21.5, 20.0 ppm; HRMS (ESI+): $\mathrm{m} / \mathrm{z}$ calcd for $\mathrm{C}_{14} \mathrm{H}_{9} \mathrm{~F}_{3} \mathrm{O}_{2}[\mathrm{M}+\mathrm{Na}]^{+}$ 289.0447 , found 289.0450 .

$5^{\prime}, 6^{\prime}, 7^{\prime}$-Trifluoro DHX-hemicyanine fused dye precursor (1) To 5,6,7trifluoro-2,3-dihydro- $1 \mathrm{H}$-xanthene-4-carbaldehyde 5 (265 mg, 0.99 mmol, 1 equiv) in EtOH ( $2 \mathrm{~mL}$ ) was added 1,2,3,3-tetramethyl-3Hindol-1-ium iodide 6 ( $300 \mathrm{mg}, 0.99 \mathrm{mmol}, 1$ equiv) and the solution was refluxed at $80^{\circ} \mathrm{C}$ for $4 \mathrm{~h}$. The reaction mixture was concentrated and the crude product was purified by flash-column chromatography on silica gel ( $1 \% \mathrm{MeOH}$ in $\mathrm{CH}_{2} \mathrm{Cl}_{2}$ ) to afford compound 1 as a dark blue solid (483 mg, 88\%). ${ }^{1} \mathrm{H}$ NMR (400.0 MHz, DMSO- $\left.d_{6}\right): \delta=8.50$ (d, $J=$ $15.6 \mathrm{~Hz}, 1 \mathrm{H}), 7.82(\mathrm{~m}, 2 \mathrm{H}), 7.56(\mathrm{~m}, 3 \mathrm{H}), 7.18(\mathrm{~s}, 1 \mathrm{H}), 6.83(\mathrm{~d}, J=15.6$ $\mathrm{Hz}, 1 \mathrm{H}), 4.01(\mathrm{~s}, 3 \mathrm{H}), 2.70(\mathrm{~m}, 4 \mathrm{H}), 1.82(\mathrm{~m}, 2 \mathrm{H}), 1.74(\mathrm{~s}, 6 \mathrm{H}) \mathrm{ppm} ;{ }^{19} \mathrm{~F}$ NMR (376.5 MHz, DMSO- $\left.d_{6}\right): \delta=-140.2(m, 1 F),-156.1(m, 1 F),-157.6$ $\left(d, J_{F-F}=19.4 \mathrm{~Hz}, 1 \mathrm{~F}\right) \mathrm{ppm} ;{ }^{13} \mathrm{C}$ NMR $\left(100.0 \mathrm{MHz}, \mathrm{DMSO}-d_{6}\right): \delta=180.0$, $156.0,146.8\left(\mathrm{dd}, J_{\mathrm{C}-\mathrm{F}}=10.2 \mathrm{~Hz},{ }^{1} J_{\mathrm{C}-\mathrm{F}}=244.2 \mathrm{~Hz}\right.$ ), 145.3, 143.1, 142.5, $140.6(\mathrm{~m}), 140.4$ (ddd, $J_{\mathrm{C}-\mathrm{F}}=11.9 \mathrm{~Hz}, J_{\mathrm{C}-\mathrm{F}}=17.1 \mathrm{~Hz},{ }^{1} J_{\mathrm{C}-\mathrm{F}}=253.0 \mathrm{~Hz}$ ), $138.0(\mathrm{~m}), 131.9,129.4,128.8,127.2,123.3,118.5$ (dd, $J_{\mathrm{C}-\mathrm{F}}=3.1 \mathrm{~Hz}$, $\left.J_{C-F}=9.2 \mathrm{~Hz}\right), 115.5,114.8,109.6\left(\mathrm{~d}, J_{\mathrm{C}-}=2.8 \mathrm{~Hz}\right), 109.4,51.6,34.1$, 29.2, 27.1, 23.9, 20.1 ppm; HRMS (ESI+): $\mathrm{m} / \mathrm{z}$ calcd for $\mathrm{C}_{26} \mathrm{H}_{23} \mathrm{~F}_{3} \mathrm{NO}^{+}$ $[\mathrm{M}]^{{ }^{\circ}} 422.1763$, found 422.1707 .

General procedure for the synthesis of NIR $5^{\prime}, 7^{\prime}$-difluoro DHXhemicyanine fused dyes $2 \mathrm{a}-2 \mathrm{v}$ and 8 : To intermediate $\mathbf{1}(220 \mathrm{mg}, 0.4$ mmol, 1 equiv) in pyridine $(1 \mathrm{~mL})$ was added the respective nucleophile ( $2 \mathrm{mmol}, 5$ equiv) and the mixture was heated at $80^{\circ} \mathrm{C}$ for $12 \mathrm{~h}$ in a sealed tube. After cooling down to r.t., the reaction mixture was concentrated in vacuo and directly purified by flashcolumn chromatography on silica gel $\left(1 \% \mathrm{MeOH}\right.$ in $\left.\mathrm{CH}_{2} \mathrm{Cl}_{2}\right)$ to afford the corresponding blue products $2 \mathbf{a}-\mathbf{2} \mathbf{v}$ and $\mathbf{8}$.

\section{Computational details}

We have followed a computational protocol developed especially for the nor-DHX-hemicyanine fused dyes and detailed in a previous published publication. ${ }^{32}$ We refer the interested reader to that earlier work for details and justification of this protocol that combines TD-DFT and second-order CC (CC2) methods. All DFT and TD-DFT calculations were performed with the Gaussian16 program, ${ }^{34}$ applying both a tightened selfconsistent field convergence criterion (10-9-10-10 a.u.) and an improved optimization threshold $\left(10^{-5} \mathrm{a}\right.$.u. on average residual forces). The ground $\left(S_{0}\right)$ and excited $\left(S_{1}\right)$ state structures of all dyes have been optimised at the CAM-B3LYP/6-311G(2d,p) level, 35 and the minima nature of all structures was confirmed using analytical calculations of the Hessian. These structural and vibrational parameters have been obtained in solution $\left(\mathrm{CHCl}_{3}\right)$, and the medium effects were evaluated with the well-known Polarizable Continuum Model (PCM) ${ }^{36}$ applying the LinearResponse (LR) ${ }^{37}$ formalism in its the equilibrium limit. The zeropoint energies of both states was computed at this same PCMCAM-B3LYP/6-311G(2d,p) level. We next determined the total and transition energies at the PCM-(TD-)CAM-B3LYP/6$311++\mathrm{G}(2 \mathrm{df}, 2 \mathrm{pd})$ level in both gas-phase and in solution, using the same LR-PCM model for the latter, considering both the non-equilibrium and equilibrium limits. CC2/aug-cc-pVTZ total and transition energies have been computed in the gas-phase on the DFT and TD-DFT structures with the Turbomole package. 38 We applied the Resolution-of-Identity (RI-V) approximation during all calculations and selected default Turbomole convergence thresholds.

To obtain the $0-0$ energies, we applied the following equation: ${ }^{39}$

$$
\begin{aligned}
& E_{\text {gas }}^{\text {adia }}[\mathrm{CC} 2]+\Delta E_{\mathrm{LR}(\mathrm{eq})}^{\mathrm{ZPVE}}[\mathrm{TDDFT}] \\
& E^{0-0}=+E_{\mathrm{LR}(\mathrm{eq})}^{\mathrm{adia}}[\mathrm{TDDFT}]-E_{\text {gas }}^{\text {adia }}[\mathrm{TDDFT}] \\
& +E_{\mathrm{LR}(\text { neq })}^{\mathrm{abso}}[\mathrm{TDDFT}]-E_{\mathrm{LR}(\mathrm{eq})}^{\mathrm{abso}}[\mathrm{TDDFT}]
\end{aligned}
$$

in which we use the so-called adiabatic energies and correct them for zero-point vibrational effects as well as for non-equilibrium solvation effects. This protocol was shown to deliver accurate results for norDHX dyes. ${ }^{32}$

\section{Photophysical properties}

UV-visible spectra were obtained either on a Varian Cary 50 scan (single-beam) or an Agilent Technologies 60 (single-beam) spectrophotometer by using a rectangular quartz cell (Hellma, 100QS, $45 \times 12.5 \times 12.5 \mathrm{~mm}$, pathlength: $10 \mathrm{~mm}$, chamber volume: 3.5 $\mathrm{mL}$ ), at $25^{\circ} \mathrm{C}$ (using a temperature control system combined with water circulation). Fluorescence spectra (emission/excitation spectra) were recorded with an HORIBA Jobin Yvon Fluorolog spectrophotometer (software FluorEssence) at $25{ }^{\circ} \mathrm{C}$ (using a temperature control system combined with water circulation), using a standard fluorometer cell (Labbox, LB Q, light path: $10 \mathrm{~mm}$, width: $10 \mathrm{~mm}$, chamber volume: $3.5 \mathrm{~mL}$ ). Emission spectra were recorded in the range $630-900 \mathrm{~nm}$ after excitation at $615 \mathrm{~nm}$ (shutter: Auto Open, excitation slit $=5 \mathrm{~nm}$ and emission slit $=5 \mathrm{~nm}$ ). Excitation spectra were recorded in the range $400-750 \mathrm{~nm}$ or $400-770 \mathrm{~nm}$ after emission at $760 \mathrm{~nm}$ or $800 \mathrm{~nm}$ (shutter: Auto Open, excitation slit = $12 \mathrm{~nm}$ and emission slit $=5 \mathrm{~nm}$ ). All fluorescence spectra were corrected until $850 \mathrm{~nm}$. Fluorescence quantum yields were measured at $25{ }^{\circ} \mathrm{C}$ by a relative method using sulfoindocyanine dye Cy $5.0\left(\phi_{\mathrm{F}}=20 \%\right.$ in PBS) as a standard (dilution by a factor of 3 between absorption and fluorescence measurements). ${ }^{40}$ The following equation was used to determine the relative fluorescence quantum yield:

$$
\phi_{F}(x)=\left(A_{S} / A_{X}\right)\left(F_{X} / F_{S}\right)\left(n_{X} / n_{S}\right)^{2} \phi_{F}(s)
$$

where $A$ is the absorbance (in the range of 0.01-0.1 A.U.), $F$ is the area under the emission curve, $\mathrm{n}$ is the refractive index of the solvents (at $25^{\circ} \mathrm{C}$ ) used in measurements, and the subscripts $s$ and $x$ represent standard and unknown, respectively. The following refractive index values were used: 1.479 for DMSO, $1.446 \mathrm{CHCl}_{3}$, $1.361 \mathrm{EtOH}, 1.337$ for PBS (100 mM phosphate $+150 \mathrm{mM} \mathrm{NaCl}, \mathrm{pH}$ 7.4) and PBS $+5 \%$ BSA. Stock solutions $(1.0 \mathrm{mg} / \mathrm{mL})$ of $5^{\prime}, 7^{\prime}$-difluoro DHX-hemicyanine fused NIR dyes were prepared in DMSO (RPE, for analysis, Carlo Erba) and subsequently diluted with $\mathrm{CHCl}_{3}, \mathrm{EtOH}$ or PBS + 5\% BSA for UV-vis absorption and fluorescence measurements.

\section{Conflicts of interest}

The authors declare no conflict of interest.

\section{Acknowledgements}

This work is supported by SPST, Tianjin University (P.R. China), ICES, A*STAR (Singapore), and the CNRS, Université de Bourgogne and 
Conseil Régional de Bourgogne through the "Plan d'Actions Régional pour I'Innovation (PARI) and the "Fonds Européen de Développement Régional (FEDER)" programs. Financial support from Institut Universitaire de France (IUF, 2013-2018), Institut Français de Singapour (PHC Merlion grant, 2015, 5.04.15) and GDR CNRS "Agents d'Imagerie Moléculaire" (AIM) 2037 are also greatly acknowledged. The authors thank Ms Doris Tan (ICES, Singapore) and Dr. Quentin Bonnin (IR CNRS, PACSMUB, Dijon, France) for the recording of HRMS spectra. A. R. thanks the "Plateforme d'Analyse Chimique et de Synthèse Moléculaire de I'Université de Bourgogne" (PACSMUB, http://www.wpcm.fr) for access to spectroscopy instrumentation. This work used the computational ressources of the CCIPL (Centre de Calcul Intensif des Pays de la Loire) installed in Nantes.

\section{Notes and references}

1 D. O'Hagan, Chem. Soc. Rev., 2008, 37, 308.

2 (a) H.-J. Böhm, D. Banner, S. Bendels, M. Kansy, B. Kuhn, K. Müller, U. Obst-Sander and M. Stahl, ChemBioChem, 2004, 5, 637; (b) K. Müller, C. Faeh and F. Diederich, Science, 2007, 317, 1881; (c) W. K. Hagmann, J. Med. Chem., 2008, 51, 4359; (d) S. Purser, P. R. Moore, S. Swallow and V. Gouverneur, Chem. Soc. Rev., 2008, 37, 320; (e) D. O'Hagan, J. Fluorine Chem., 2010, 131, 1071; (f) E. P. Gillis, K. J. Eastman, M. D. Hill, D. J. Donnelly and N. A. Meanwell, J. Med. Chem., 2015, 58, 8315; (g) N. A. Meanwell, J. Med. Chem., 2018, 61, 5822.

3 (a) P. Jeschke, ChemBioChem, 2004, 5, 570; (b) P. Jeschke, Pest Manage. Sci., 2010, 66, 10; (c) S. Jeanmart, A. J. F. Edmunds, C. Lamberth and M. Pouliot, Bioorg. Med. Chem., 2016, 24, 317.

4 R. Berger, G. Resnati, P. Metrangolo, E. Weber and J. Hulliger, Chem. Soc. Rev., 2011, 40, 3496.

5 F. Babudri, G. M. Farinola, F. Naso and R. Ragni, Chem. Commun., 2007, 1003.

6 D. Anton, Adv. Mater., 1998, 10, 1197.

7 (a) J. Wang, M. Sánchez-Roselló, J. L. Aceña, C. del Pozo, A. E. Sorochinsky, S. Fustero, V. A. Soloshonok and H. Liu, Chem. Rev., 2014, 114, 2432; (b) Y. Zhou, J. Wang, Z. Gu, S. Wang, W. Zhu, J. L. Aceña, V. A. Soloshonok, K. Izawa and H. Liu, Chem. Rev., 2016, 116, 422.

8 T. Fujiwara and D. O'Hagan, J. Fluorine Chem., 2014, 167, 16.

9 (a) L. Cai, S. Lu and V. W. Pike, Eur. J. Org. Chem., 2008, 2853; (b) D. van der Born, A. Pees, A. J. Poot, R. V. A. Orru, A. D. Windhorst and D. J. Vugts, Chem. Soc. Rev., 2017, 46, 4709.

10 I. Tirotta, V. Dichiarante, C. Pigliacelli, G. Cavallo, G. Terraneo, F. B. Bombelli, P. Metrangolo and G. Resnati, Chem. Rev., 2015, 115, 1106.

11 (a) H. Chen, S. Viel, F. Ziarelli and L. Peng, Chem. Soc. Rev., 2013, 42, 7971; (b) E. N. G. Marsh and Y. Suzuki, ACS Chem. Biol., 2014, 9, 1242 .

12 (a) D. P. Curran, Angew. Chem. Int. Ed., 1998, 37, 1174; (b) D. P. Curran and Z. Luo, J. Am. Chem. Soc., 1999, 121, 9069; (c) D. P. Curran, Synlett, 2001, 1488; (d) W. Zhang, Tetrahedron, 2003, 59, 4475.

13 M. Matsui, in Functional Dyes, Elsevier Science, Amsterdam, 2006, pp. 257.

14 D. K. Kölmel, B. Rudat, D. M. Braun, C. Bednarek, U. Schepers and S. Bräse, Org. Biomol. Chem., 2013, 11, 3954.

15 E. M. Sletten and T. M. Swager, J. Am. Chem. Soc., 2014, 136, 13574.

16 W. Cao and E. M. Sletten, J. Am. Chem. Soc., 2018, 140, 2727.
17 (a) V. N. Belov, M. L. Bossi, J. Fölling, V. P. Boyarskiy and S. W. Hell, Chem. Eur. J., 2009, 15, 10762; (b) G. Y. Mitronova, V. N. Belov, M. L. Bossi, C. A. Wurm, L. Meyer, R. Medda, G. Moneron, S. Bretschneider, C. Eggeling, S. Jakobs and S. W. Hell, Chem. Eur. J., 2010, 16, 4477; (c) K. Kolmakov, V. N. Belov, J. Bierwagen, C. Ringemann, V. Müller, C. Eggeling and S. W. Hell, Chem. Eur. J., 2010, 16, 158; (d) Z. R. Woydziak, L. Fu and B. R. Peterson, J. Org. Chem., 2012, 77, 473; (e) H. Schill, S. Nizamov, F. Bottanelli, J. Bierwagen, V. N. Belov and S. W. Hell, Chem. Eur. J., 2013, 19, 16556.

18 (a) B. J. Littler, M. A. Miller, C.-H. Hung, R. W. Wagner, D. F. O'Shea, P. D. Boyle and J. S. Lindsey, J. Org. Chem., 1999, 64, 1391; (b) M. A. H. Alamiry, A. C. Benniston, J. Hagon, T. P. L. Winstanley, H. Lemmetyinen and N. V. Tkachenko, RSC Adv., 2012, 2, 4944; (c) M. Hecht, T. Fischer, P. Dietrich, W. Kraus, A. B. Descalzo, W. E. S. Unger and K. Rurack, ChemistryOpen, 2013, 2, 25.

19 (a) J. Lenaerts, G. Verlinden, L. Van Vaeck, R. Gijbels, I. Geuens and P. Callant, Langmuir, 2001, 17, 7332; (b) B. R. Renikuntla, H. C. Rose, J. Eldo, A. S. Waggoner and B. A. Armitage, Org. Lett., 2004, 6, 909; (c) G. L. Silva, V. Ediz, D. Yaron and B. A. Armitage, J. Am. Chem. Soc., 2007, 129, 5710.

20 Cooper, M. E.; Gardner, N. J.; Laughton, P. G. (Amersham, GB). US Patent 7,767,829 B2, 2010.

21 (a) R. Ting, T. A. Aguilera, J. L. Crisp, D. J. Hall, W. C. Eckelman, D. R. Vera and R. Y. Tsien, Bioconjugate Chem., 2010, 21, 1811; (b) T. Priem, C. Bouteiller, D. Camporese, X. Brune, J. Hardouin, A. Romieu and P.-Y. Renard, Org. Biomol. Chem., 2013, 11, 469; (c) E. A. Rodriguez, Y. Wang, J. L. Crisp, D. R. Vera, R. Y. Tsien and R. Ting, Bioconjugate Chem., 2016, 27, 1390; (d) F.-F. An, H. Kommidi, N. Chen and R. Ting, Int. J. Mol. Sci., 2017, 18, 1214.

22 G. Vives, C. Giansante, R. Bofinger, G. Raffy, A. D. Guerzo, B. Kauffmann, P. Batat, G. Jonusauskas and N. D. McClenaghan, Chem. Commun., 2011, 47, 10425.

23 H. R. A. Golf, H.-U. Reissig and A. Wiehe, Org. Lett., 2015, 17, 982.

24 (a) R. Weissleder and U. Mahmood, Radiology, 2001, 219, 316; (b) J. V. Frangioni, Curr. Opin. Chem. Biol., 2003, 7, 626; (c) I. Martinić, S. V. Eliseeva and S. Petoud, J. Lumin., 2017, 189, 19.

25 O. Galangau, C. Dumas-Verdes, R. Méallet-Renault and G. Clavier, Org. Biomol. Chem., 2010, 8, 4546.

26 For mono-fluorinated phenol-based DHX fluorophores with a lowered pKa value promoting a strong NIR emissive phenolate form at physiological pH, see: (a) Q. Yang, C. Jia, Q. Chen, W. Du, Y. Wang and Q. Zhang, J. Mater. Chem. B, 2017, 5, 2002; (b) X. Zhu, L. Yuan, X. Hu, L. Zhang, Y. Liang, S. He, X.-B. Zhang and W. Tan, Sens Actuators B Chem., 2018, 259, 219.

27 H. Chen, B. Dong, Y. Tang and W. Lin, Acc. Chem. Res., 2017, 50, 1410.

28 (a) J.-A. Richard, Org. Biomol. Chem., 2015, 13, 8169; (b) M. J. H. Ong, R. Srinivasan, A. Romieu and J.-A. Richard, Org. Lett., 2016, 18, 5122; (c) A. Romieu and J.-A. Richard, Tetrahedron Lett., 2016, 57, 317; (d) M. J. H. Ong, S. Debieu, M. Moreau, A. Romieu and J.-A. Richard, Chem. Asian J., 2017, 12, 936.

29 J. Pauli, M. Grabolle, R. Brehm, M. Spieles, F. M. Hamann, M. Wenzel, I. Hilger and U. Resch-Genger, Bioconjugate Chem., 2011, 22, 1298.

30 M. R. Detty, P. N. Prasad, D. J. Donnelly, T. Ohulchanskyy, S. L. Gibson and R. Hilf, Bioorg. Med. Chem., 2004, 12, 2537.

31 T. Myochin, K. Hanaoka, S. Iwaki, T. Ueno, T. Komatsu, T. Terai, T. Nagano and Y. Urano, J. Am. Chem. Soc., 2015, 137, 4759.

32 C. Azarias, M. Ponce-Vargas, I. Navizet, P. Fleurat-Lessard, A. Romieu, B. Le Guennic, J.-A. Richard and D. Jacquemin, Phys. Chem. Chem. Phys., 2018, 20, 12120. 
33 For selected examples of small molecule theranostics based on DHX-hemicyanine hybrids or related NIR fluorophores, see: (a) X. Wu, M. Yu, B. Lin, H. Xing, J. Han and S. Han, Chem. Sci., 2015, 6, 798; (b) F. Kong, Z. Liang, D. Luan, X. Liu, K. Xu and B. Tang, Anal. Chem., 2016, 88, 6450; (c) B. Zhou, Y. Li, G. Niu, M. Lan, Q. Jia and Q. Liang, ACS Appl. Mater. Interfaces, 2016, 8, 29899.

34 M. J. Frisch, G. W. Trucks, H. B. Schlegel, G. E. Scuseria, M. A. Robb, J. R. Cheeseman, G. Scalmani, V. Barone, G. A. Petersson, H. Nakatsuji, X. Li, M. Caricato, A. V. Marenich, J. Bloino, B. G. Janesko, R. Gomperts, B. Mennucci, H. P. Hratchian, J. V. Ortiz, A. F. Izmaylov, J. L. Sonnenberg, D. Williams-Young, F. Ding, F. Lipparini, F. Egidi, J. Goings, B. Peng, A. Petrone, T. Henderson, D. Ranasinghe, V. G. Zakrzewski, J. Gao, N. Rega, G. Zheng, W. Liang, M. Hada, M. Ehara, K. Toyota, R. Fukuda, J. Hasegawa, M. Ishida, T. Nakajima, Y. Honda, O. Kitao, H. Nakai, T. Vreven, K. Throssell, J. A. Montgomery Jr., J. E. Peralta, F. Ogliaro, M. J. Bearpark, J. J. Heyd, E. N. Brothers, K. N. Kudin, V. N. Staroverov, T. A. Keith, R. Kobayashi, J. Normand, K. Raghavachari, A. P. Rendell, J. C. Burant, S. S. Iyengar, J. Tomasi, M. Cossi, J. M. Millam, M. Klene, C. Adamo, R. Cammi, J. W. Ochterski, R. L. Martin, K. Morokuma, O. Farkas, J. B. Foresman and D. J. Fox, Gaussian 16 Revision A.03, Gaussian Inc., Wallingford CT, 2016.

35 T. Yanai, D. P. Tew and N. C. Handy, Chem. Phys. Lett., 2004, 393, 51.

36 J. Tomasi, B. Mennucci and R. Cammi, Chem. Rev., 2005, 105, 2999.

37 R. Cammi and B. Mennucci, J. Chem. Phys., 1999, 110, 9877.

38 TURBOMOLE V6.6 2014, a development of University of Karlsruhe and Forschungszentrum Karlsruhe $\mathrm{GmbH}$, 1989-2007, TURBOMOLE GmbH, since 2007, available from http://www.turbomole.com, accessed 13 June 2016.

39 Note that $\mathrm{Eq}(3)$ in Ref. 32 was incorrect, but the calculations in that earlier work have been actually performed with this (correct) formalism.

40 R. B. Mujumdar, L. A. Ernst, S. R. Mujumdar, C. J. Lewis and A. S. Waggoner, Bioconjugate Chem., 1993, 4, 105. 


\section{Table of contents}

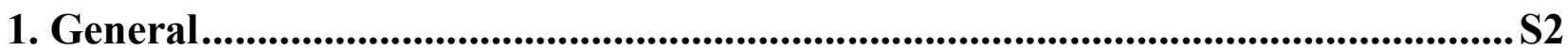

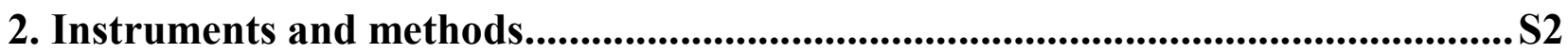

3. Experimental Section ..................................................................................................... S2

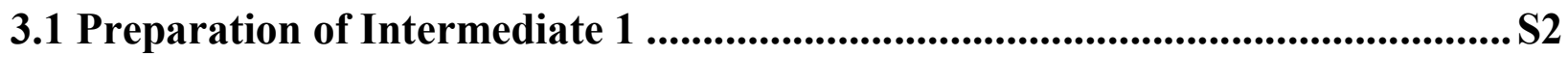

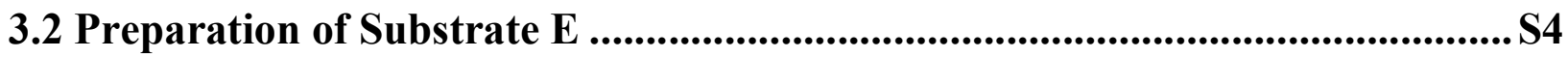

3.3 General procedure for the $S_{N} A r$ reaction.................................................................S5

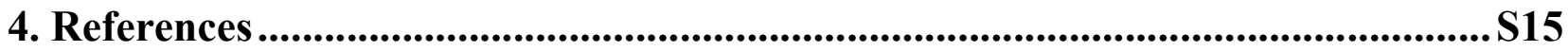

5. ${ }^{1} \mathrm{H},{ }^{19} \mathrm{~F}$ and ${ }^{13} \mathrm{C}$ NMR spectra of synthesized compounds .....................................S16

6. High-performance liquid chromatography (HPLC) separations ....................... S57

7. UV-visible absorption spectra of compound 1 and non-fluorescent 5',7'-difluoro-

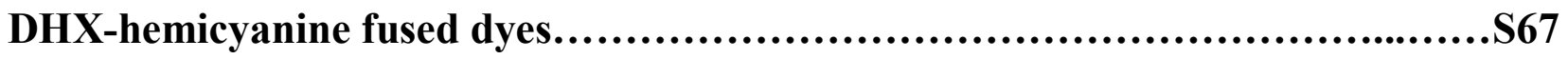

8. Absorption, excitation and emission spectra of selected 5',7'-difluoro-6'-aminoDHX-hemicyanine fused dyes..................................................568 


\section{Supporting Information}

\section{General}

Chemicals, reagents and solvents were purchased from commercial vendors and used without further purification. Trifluoroacetic acid (TFA), dichloromethane (DCM), dimethylformamide (DMF), ethyl acetate (EtOAc), petroleum ether (b.p. $40-60^{\circ} \mathrm{C}$ ), acetonitrile (MeCN), dimethyl sulfoxide (DMSO), methanol (MeOH), ethanol ( $\left.\mathrm{EtOH}\right)$, and pyridine used in this work were reagent grade. Bovine serum albumin (BSA, heat shock fraction, $\mathrm{pH} 7, \geq 98 \%$ ) was provided by SigmaAldrich. The HPLC-gradient grade $\mathrm{CH}_{3} \mathrm{CN}$ used for HPLC-MS analyses was obtained from Carlo Erba. Formic acid (FA, puriss p.a., ACS reagent, reag. Ph. Eur., $\geq 98 \%$ ) was provided by Sigma-Aldrich. All aq. buffers used in this work (PBS and aq. mobile-phases for HPLC were prepared using water purified with a PURELAB Ultra system from ELGA (purified to $18.2 \mathrm{M} \Omega . \mathrm{cm}$ ). Thin-layer chromatography (TLC) was carried on pre-coated glass plates with $0.2 \mathrm{~mm}$ silica gel and visualized under UV $(254 \mathrm{~nm})$ and/or potassium permanganate $\left(\mathrm{KMnO}_{4}\right)$ stain. Flash-column chromatography was performed using Silica gel 60 (200-400 mesh) with specified eluents.

\section{Instruments and methods}

${ }^{1} \mathrm{H},{ }^{13} \mathrm{C}$ and ${ }^{19} \mathrm{~F}$ NMR spectra were recorded on Brüker Avance $400 \mathrm{MHz}$ or $600 \mathrm{MHz}$ spectrometers. Chemical shifts $(\delta)$ are reported in parts per million (ppm) and residual non-deuterated solvent peaks were used as internal reference (proton $\delta 7.26$ and carbon $\delta 77.16$ for $\mathrm{CDCl}_{3}$ and proton $\delta 2.50$ and carbon $\delta 39.5$ for DMSO- $d_{6}$ ). ${ }^{1} \mathrm{H}$ NMR coupling constants $(J)$ are reported in Hertz $(\mathrm{Hz})$. The following abbreviations were used in reporting multiplicities: s (singlet), d (doublet), $\mathrm{t}$ (triplet), $\mathrm{m}$ (multiplet), dd (doublet of doublets), ddd (doublet of doublet of doublets) and br (broad). High-resolution mass spectra (HRMS) were recorded on either a Q-ToF micro (Bruker Compass Data Analysis 4.0) spectrometer or a Thermo LTQ Orbitrap XL apparatus, both equipped with an ESI analytical source. HPLC-MS analyses were performed on a Thermo-Dionex Ultimate 3000 instrument (pump + autosampler at $20^{\circ} \mathrm{C}+$ column oven at $25^{\circ} \mathrm{C}$ ) equipped with a diode array detector (Thermo-Dionex DAD 3000-RS) and a MSQ Plus single quadrupole mass spectrometer (lowresolution mass (LRMS) analyses through electrospray ionization (ESI) source).

\section{Experimental Section}

\subsection{Preparation of Intermediate 1}

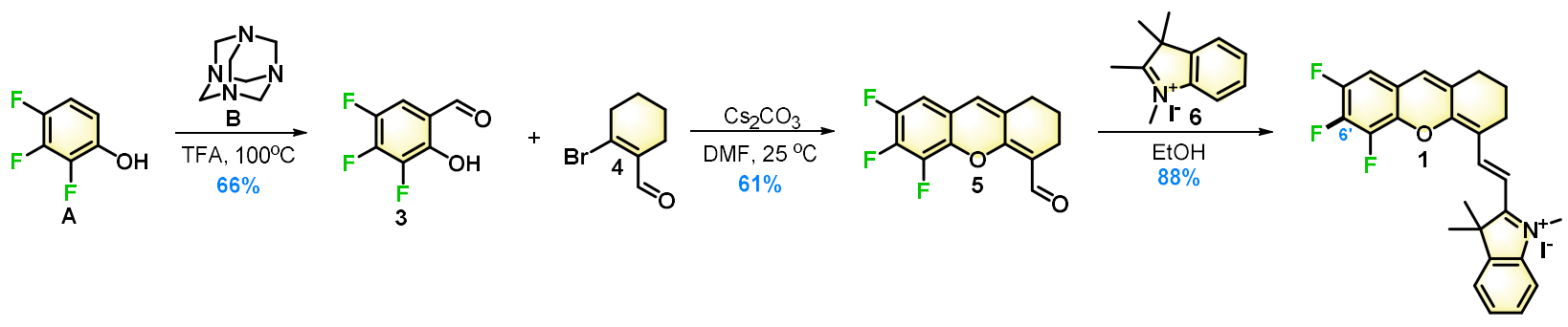

Scheme S1 Synthetic route towards Intermediate 1 


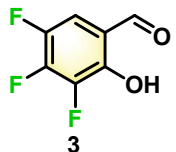

2-Hydroxyl-3,4,5-trifluoro-benzaldehyde 3. To 2,3,4-trifluorophenol A (148 mg, $1 \mathrm{mmol}, 1$ equiv.) in TFA (2 mL) was added hexamethylenetetramine $\mathbf{B}$ (350 mg, $2.5 \mathrm{mmol}, 2.5$ equiv.). The mixture was stirred at room temperature (r.t.) for $\sim 10$ min until observation of a clear solution. The resulting solution was refluxed at $100{ }^{\circ} \mathrm{C}$ for $13 \mathrm{~h}$ and cooled down to r.t. Deionized water $(2 \mathrm{~mL})$ and conc. $\mathrm{H}_{2} \mathrm{SO}_{4}(370 \mu \mathrm{L})$ were added and the mixture was further stirred at r.t. for $18 \mathrm{~h}$. The reaction mixture was extracted with DCM $(3 \times 20 \mathrm{~mL})$ and the combined organic layers were dried over anhydrous $\mathrm{Na}_{2} \mathrm{SO}_{4}$, filtered and concentrated in vacuo. Purification by flash-column chromatography on silica gel (100\% Petroleum ether) afforded 2-hydroxyl-3,4,5-trifluoro-benzaldehyde 3 as a white solid (117 mg, 66\%). ${ }^{1} \mathrm{H} \mathrm{NMR}\left(400.0 \mathrm{MHz}^{\mathrm{CDCl}}\right)_{3}$ : $\delta=11.05(\mathrm{~s}, 1 \mathrm{H}), 9.83(\mathrm{~s}, 1 \mathrm{H}), 7.27(\mathrm{~m}, 1 \mathrm{H}) \mathrm{ppm} ;{ }^{19} \mathrm{~F} \mathrm{NMR}\left(376.5 \mathrm{MHz}, \mathrm{CDCl}_{3}\right): \delta=-144.4(\mathrm{dd}, J=9.0,21.1 \mathrm{~Hz}, 1 \mathrm{~F})$, $-145.2(\mathrm{~m}, 1 \mathrm{~F}),-155.3(\mathrm{~d}, J=18.3 \mathrm{~Hz}, 1 \mathrm{~F}) \mathrm{ppm} ;{ }^{13} \mathrm{C} \mathrm{NMR}\left(100.0 \mathrm{MHz}, \mathrm{CDCl}_{3}\right): \delta=194.7,148.2(\mathrm{~m}), 145.7\left(\mathrm{ddd}, J_{\mathrm{C}-\mathrm{FC}}\right.$ $\left.=12.3 \mathrm{~Hz}, J_{\mathrm{C}-\mathrm{F}}=16.2 \mathrm{~Hz},{ }^{1} J_{\mathrm{C}-\mathrm{F}}=262.0 \mathrm{~Hz}\right), 144.4\left(\mathrm{ddd}, J_{\mathrm{C}-\mathrm{F}}=1.2 \mathrm{~Hz}, J_{\mathrm{C}-\mathrm{F}}=10.8 \mathrm{~Hz},{ }^{1} J_{\mathrm{C}-\mathrm{F}}=245.4 \mathrm{~Hz}\right), 140.4\left(\mathrm{ddd}, J_{\mathrm{C}-}\right.$ $\left.\mathrm{F}=2.6 \mathrm{~Hz}, J_{\mathrm{C}-\mathrm{F}}=11.8 \mathrm{~Hz},{ }^{1} J_{\mathrm{C}-\mathrm{F}}=254.5 \mathrm{~Hz}\right), 115.7(\mathrm{~m}), 114.0\left(\mathrm{ddd}, J_{\mathrm{C}-\mathrm{F}}=1.9 \mathrm{~Hz}, J_{\mathrm{C}-\mathrm{F}}=3.7 \mathrm{~Hz}, J_{\mathrm{C}-\mathrm{F}}=18.1 \mathrm{~Hz}\right) \mathrm{ppm}$; MS (EI+): $\mathrm{m} / \mathrm{z}$ calcd for $\mathrm{C}_{7} \mathrm{H}_{3} \mathrm{~F}_{3} \mathrm{O}_{2}[\mathrm{M}]+176.0085$, found 176.0000 .

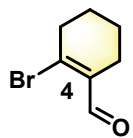

6-Bromocyclohex-1-ene-1-carbaldehyde 4. To a mixture of DMF (23.5 mL, $306 \mathrm{mmol}, 3$ equiv.) and $\mathrm{CHCl}_{3}(100 \mathrm{~mL})$ at $0{ }^{\circ} \mathrm{C}$ was added $\mathrm{PBr}_{3}\left(24.2 \mathrm{~mL}, 255 \mathrm{mmol}, 2.5\right.$ equiv.) portionwise under an atmosphere of $\mathrm{N}_{2}$. After $1.5 \mathrm{~h}$, cyclohexanone $(10.5 \mathrm{~mL}, 102 \mathrm{mmol}, 1$ equiv.) was added and the mixture was stirred at r.t. overnight. The resulting solution was poured onto ice and then solid $\mathrm{NaHCO}_{3}$ was slowly added until $\mathrm{pH} \sim 7$. The aqueous layer was extracted with $\mathrm{CH}_{2} \mathrm{Cl}_{2}$ and the organic layer was washed with water. The combined organic layers were dried over anhydrous $\mathrm{Na}_{2} \mathrm{SO}_{4}$ and concentrated. The yellow mixture was purified by flash-column chromatography on silica gel (100\% Petroleum ether) and 6-Bromocyclohex-1-ene-1-carbaldehyde 4 was obtained as a pale yellow liquid (13.6 g, $72 \%)$. ${ }^{1} \mathrm{H}$ $\operatorname{NMR}\left(400.0 \mathrm{MHz}, \mathrm{CDCl}_{3}\right): \delta=10.01(\mathrm{~s}, 1 \mathrm{H}), 2.74(\mathrm{~m}, 2 \mathrm{H}), 2.26(\mathrm{~m}, 2 \mathrm{H}), 1.75(\mathrm{~m}, 2 \mathrm{H}), 1.68(\mathrm{~m}, 2 \mathrm{H})$. The spectral data matched with the reference. ${ }^{1}$

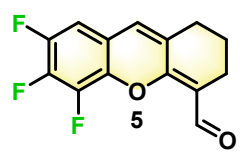

5',6',7'-Trifluoro-2,3-dihydro-1H-xanthene-4-carbaldehyde 5. To 2-hydroxyl-3,4,5-trifluoro-benzaldehyde 3 (528 mg, 3 mmol, 1 equiv.) and $\mathrm{Cs}_{2} \mathrm{CO}_{3}$ (978 mg, $3 \mathrm{mmol}, 1$ equiv.) in DMF (15 mL) was added 6-bromocyclohex-1-ene-1carbaldehyde 4 (2.26 g, $12 \mathrm{mmol}, 4$ equiv.) and the mixture was stirred at $25{ }^{\circ} \mathrm{C}$ for $56 \mathrm{~h}$. The reaction mixture was filtered and concentrated in vacuo. Purification by flash-column chromatography on silica gel (10\% EtOAc in petroleum ether) afforded 5,6,7-Trifluoro-2,3-dihydro-1H-xanthene-4-carbaldehyde 5 as a yellow solid (483 mg, $61 \%) .{ }^{1} \mathrm{H} \mathrm{NMR}$ $\left(400.0 \mathrm{MHz}, \mathrm{CDCl}_{3}\right): \delta=10.35(\mathrm{~s}, 1 \mathrm{H}), 6.78(\mathrm{~m}, 1 \mathrm{H}), 6.53(\mathrm{~s}, 1 \mathrm{H}), 2.59(\mathrm{t}, J=5.8 \mathrm{~Hz}, 2 \mathrm{H}), 2.45(\mathrm{t}, J=6.1 \mathrm{~Hz}, 2 \mathrm{H}), 1.73$ $(\mathrm{m}, 2 \mathrm{H}) \mathrm{ppm} ;{ }^{19} \mathrm{~F}$ NMR $\left(376.5 \mathrm{MHz}, \mathrm{CDCl}_{3}\right): \delta=-140.7(\mathrm{~m}, 1 \mathrm{~F}),-155.2(\mathrm{~m}, 1 \mathrm{~F}),-155.8(\mathrm{~m}, 1 \mathrm{~F}) \mathrm{ppm} ;{ }^{13} \mathrm{C} \mathrm{NMR}(100.0$ $\left.\mathrm{MHz}_{\mathrm{CDCl}}\right): \delta=188.1,157.9,146.9\left(\mathrm{ddd}, J_{\mathrm{C}-\mathrm{F}}=2.1 \mathrm{~Hz}, J_{\mathrm{C}-\mathrm{F}}=11.1 \mathrm{~Hz},{ }^{1} J_{\mathrm{C}-\mathrm{F}}=246.6 \mathrm{~Hz}\right), 140.6\left(\mathrm{ddd}, J_{\mathrm{C}-\mathrm{F}}=12.1 \mathrm{~Hz}\right.$, $\left.J_{\mathrm{C}-\mathrm{F}}=16.9 \mathrm{~Hz},{ }^{1} J_{\mathrm{C}-\mathrm{F}}=254.2 \mathrm{~Hz}\right), 139.8\left(\mathrm{ddd}, J_{\mathrm{C}-\mathrm{F}}=4.0 \mathrm{~Hz}, J_{\mathrm{C}-\mathrm{F}}=12.5 \mathrm{~Hz},{ }^{1} J_{\mathrm{C}-\mathrm{F}}=253.8 \mathrm{~Hz}\right), 137.4(\mathrm{~m}), 131.5\left(\mathrm{~d}, J_{\mathrm{C}-\mathrm{F}}\right.$ $=2.9 \mathrm{~Hz}), 123.6\left(\mathrm{dd}, J_{\mathrm{C}-\mathrm{F}}=3.1 \mathrm{~Hz}, J_{\mathrm{C}-\mathrm{F}}=5.7 \mathrm{~Hz}\right), 117.1(\mathrm{~m}), 115.2\left(\mathrm{~d}, J_{\mathrm{C}-\mathrm{F}}=0.9 \mathrm{~Hz}\right), 107.8\left(\mathrm{dd}, J_{\mathrm{C}-\mathrm{F}}=3.7 \mathrm{~Hz}, J_{\mathrm{C}-\mathrm{F}}=\right.$ 19.6 Hz), 30.1, 21.5, 20.0 ppm; HRMS (ESI+): m/z calcd for $\mathrm{C}_{14} \mathrm{H}_{9} \mathrm{~F}_{3} \mathrm{O}_{2}[\mathrm{M}+\mathrm{Na}]^{+} 289.0447$, found 289.0450 . 


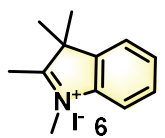

1,2,3,3-tetramethyl-3H-indol-1-ium iodide 6. To 2,3,3-trimethyl-3H-idole (32 mL, $200 \mathrm{mmol}, 1$ equiv.) in $\mathrm{CH}_{3} \mathrm{CN}(200$ $\mathrm{mL})$ was added iodomethane (14 mL, $228 \mathrm{mmol}, 2.28$ equiv.) portion-wise and the solution was refluxed overnight. The precipitate was filtered and washed with $\mathrm{Et}_{2} \mathrm{O}$ and dried in vacuo to afford 1,2,3,3-tetramethyl-3H-indol-1-ium iodide 6 as a light pink solid (56.5 g, 94\%). ${ }^{1} \mathrm{H}$ NMR (400.0 MHz, DMSO- $\left.d_{6}\right): \delta=7.91(\mathrm{~m}, 1 \mathrm{H}), 7.82(\mathrm{~m}, 1 \mathrm{H}), 7.62(\mathrm{~m}, 2 \mathrm{H}), 3.97$ (s, 3H), 2.76 (s, 3H), 1.53 (s, 3H) ppm; ${ }^{13} \mathrm{C}$ NMR (100.0 MHz, DMSO- $\left.d_{6}\right): \delta=196.5,142.6,142.1,129.8,129.3,123.8$, $115.6,54.4,35.1,22.2,14.5 \mathrm{ppm}$. The spectral data matched with the reference. ${ }^{2}$

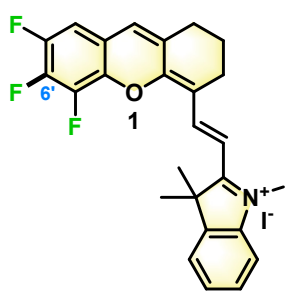

5',6',7'-trifluoro DHX-hemicyanine fused dye precursor 1. To 5',6',7'-Trifluoro-2,3-dihydro-1H-xanthene-4carbaldehyde 5 (265 mg, 0.99 mmol, 1 equiv.) in EtOH (2 mL) was added 1,2,3,3-tetramethyl-3H-indol-1-ium iodide 6 (300 mg, $0.99 \mathrm{mmol}, 1$ equiv.) and the solution was refluxed at $80^{\circ} \mathrm{C}$ for $4 \mathrm{~h}$. The reaction mixture was concentrated and the crude product was purified by flash-column chromatography on silica gel $\left(1 \% \mathrm{MeOH}\right.$ in $\left.\mathrm{CH}_{2} \mathrm{Cl}_{2}\right)$ to afford compound 1 as a dark blue solid (483 mg, 88\%). ${ }^{1} \mathrm{H}$ NMR (400.0 MHz, DMSO- $\left.d_{6}\right): \delta=8.50(\mathrm{~d}, J=15.6 \mathrm{~Hz}, 1 \mathrm{H}), 7.82(\mathrm{~m}, 2 \mathrm{H})$, $7.56(\mathrm{~m}, 3 \mathrm{H}), 7.18(\mathrm{~s}, 1 \mathrm{H}), 6.83(\mathrm{~d}, J=15.6 \mathrm{~Hz}, 1 \mathrm{H}), 4.01(\mathrm{~s}, 3 \mathrm{H}), 2.70(\mathrm{~m}, 4 \mathrm{H}), 1.82(\mathrm{~m}, 2 \mathrm{H}), 1.74(\mathrm{~s}, 6 \mathrm{H}) \mathrm{ppm} ;{ }^{19} \mathrm{~F}$ NMR (376.5 MHz, DMSO-d $): \delta=-140.2(\mathrm{~m}, 1 \mathrm{~F}),-156.1(\mathrm{~m}, 1 \mathrm{~F}),-157.6\left(\mathrm{~d}, J_{\mathrm{F}-\mathrm{F}}=19.4 \mathrm{~Hz}, 1 \mathrm{~F}\right) \mathrm{ppm} ;{ }^{13} \mathrm{C} \mathrm{NMR}(100.0$ MHz, DMSO- $\left.d_{6}\right): \delta=180.0,156.0,146.8\left(\mathrm{dd}, J_{\mathrm{C}-\mathrm{F}}=10.2 \mathrm{~Hz},{ }^{1} J_{\mathrm{C}-\mathrm{F}}=244.2 \mathrm{~Hz}\right), 145.3,143.1,142.5,140.6(\mathrm{~m}), 140.4$ $\left(\mathrm{ddd}, J_{\mathrm{C}-\mathrm{F}}=11.9 \mathrm{~Hz}, J_{\mathrm{C}-\mathrm{F}}=17.1 \mathrm{~Hz},{ }^{1} J_{\mathrm{C}-\mathrm{F}}=253.0 \mathrm{~Hz}\right), 138.0(\mathrm{~m}), 131.9,129.4,128.8,127.2,123.3,118.5\left(\mathrm{dd}, J_{\mathrm{C}-\mathrm{F}}=\right.$ $\left.3.1 \mathrm{~Hz}, J_{\mathrm{C}-\mathrm{F}}=9.2 \mathrm{~Hz}\right), 115.5,114.8,109.6\left(\mathrm{~d}, J_{\mathrm{C}-\mathrm{F}}=2.8 \mathrm{~Hz}\right), 109.4,51.6,34.1,29.2,27.1,23.9,20.1 \mathrm{ppm}$; HRMS (ESI+): $\mathrm{m} / \mathrm{z}$ calcd for $\mathrm{C}_{26} \mathrm{H}_{23} \mathrm{~F}_{3} \mathrm{NO}^{+}[\mathrm{M}]^{+\circ} 422.1763$, found 422.1707; HPLC (system A): $t_{\mathrm{R}}=4.8$ min (purity $>99 \%$ at $600 \mathrm{~nm}$ ); LRMS (ESI+, recorded during RP-HPLC analysis): $m / z 422.2[\mathrm{M}]^{+{ }^{\circ}}$ (100), calcd for $\mathrm{C}_{26} \mathrm{H}_{23} \mathrm{~F}_{3} \mathrm{NO}^{+} 422.2$; $\mathrm{UV}_{-\mathrm{vis}}$ (recorded during the HPLC analysis): $\lambda_{\max }=541 \mathrm{~nm}$ (broad peak).

\subsection{Preparation of Substrate $E$}
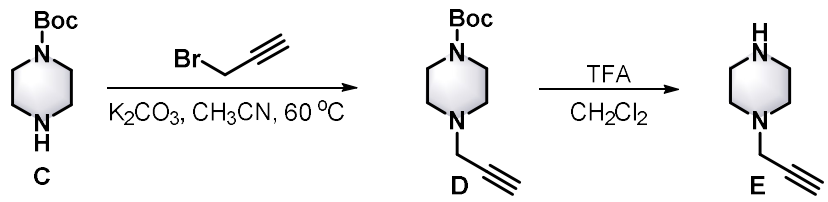

Scheme S2 Synthesis of substrate $\mathbf{E}$

To 1-(tert-Butyloxycarbonyl)piperazine $\mathbf{C}$ (3.7 g, $20 \mathrm{mmol}, 1$ equiv.) in $\mathrm{CH}_{3} \mathrm{CN}$ (80 mL) were added 3-bromopropyne ( $1.9 \mathrm{~mL}, 22 \mathrm{mmol}, 1.1$ equiv.) and $\mathrm{K}_{2} \mathrm{CO}_{3}$ (4.1 g, $30 \mathrm{mmol}, 1.5$ equiv.) and the mixture was stirred at $60^{\circ} \mathrm{C}$ for $2 \mathrm{~h}$. After cooling down to r.t., the solvent was removed under reduced pressure. The residue was washed with water and extracted 
in DCM. Purification by flash-column chromatography on silica gel (20\% EtOAc in petroleum ether) afforded tert-butyl 4-(prop-2-yn-1-yl)piperazine-1-carboxylate $\mathbf{D}$. The latter was dissolved in DCM $(25 \mathrm{~mL})$, TFA $(25 \mathrm{~mL})$ was added and the mixture was stirred at r.t. for $3 \mathrm{~h}$. The reaction mixture was washed with sat. aqueous $\mathrm{NaHCO}_{3}$ solution and extracted with EtOAc. After concentration in vacuo, 1-(prop-2-yn-1-yl)piperazine $\mathbf{E}$ was obtained as a yellow solid (1.2 g, 48\%). ${ }^{1} \mathrm{H}$ NMR (400.0 MHz, DMSO- $d_{6}$ ): $\delta=3.20$ (d, $J=2.2 \mathrm{~Hz}, 2 \mathrm{H}$ ), 3.09 (t, $\left.J=2.4 \mathrm{~Hz}, 1 \mathrm{H}\right), 2.71$ (brs, 4H), 2.36 (brs, 4H) $\mathrm{ppm}$. The spectrum matched with the corresponding reference. ${ }^{3}$

\subsection{General procedure for the $S_{N} A r$ reaction}
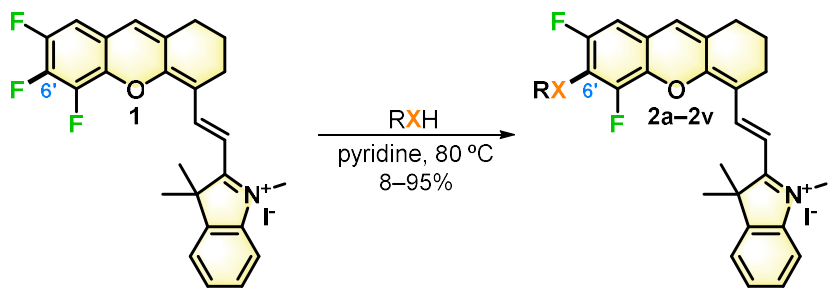

General procedure for the synthesis of NIR DHX-hemicyanine fused dyes 8 and $\mathbf{2 a}-\mathbf{2 u}$. To intermediate $\mathbf{1}(220 \mathrm{mg}$, $0.4 \mathrm{mmol}, 1$ equiv.) in pyridine $(1 \mathrm{~mL})$ was added the respective nucleophile $(2 \mathrm{mmol}, 5$ equiv.) and the mixture was heated at $80{ }^{\circ} \mathrm{C}$ for $12 \mathrm{~h}$ in a sealed tube. After cooling down to r.t., the reaction mixture was concentrated in vacuo and directly purified by flash-column chromatography on silica gel $\left(1 \% \mathrm{MeOH}\right.$ in $\left.\mathrm{CH}_{2} \mathrm{Cl}_{2}\right)$ to afford the corresponding blue products $\mathbf{2 a}-\mathbf{2 u}$.

Table 1 The product and corresponding nucleophiles

\begin{tabular}{|c|c|c|}
\hline Nucleophiles (RXH) & Product & Yields \\
\hline & 8 & $61 \%$ \\
\hline & $2 a$ & $25 \%$ \\
\hline & $2 \mathbf{b}$ & $34 \%$ \\
\hline & $2 c$ & $16 \%$ \\
\hline $\mathrm{NaN}_{3}$ & $2 d$ & $44 \%$ \\
\hline & $2 e$ & $61 \%$ \\
\hline & $2 f$ & $65 \%$ \\
\hline & $2 \mathrm{~g}$ & $8 \%$ \\
\hline & $2 \mathrm{~h}$ & $45 \%$ \\
\hline
\end{tabular}




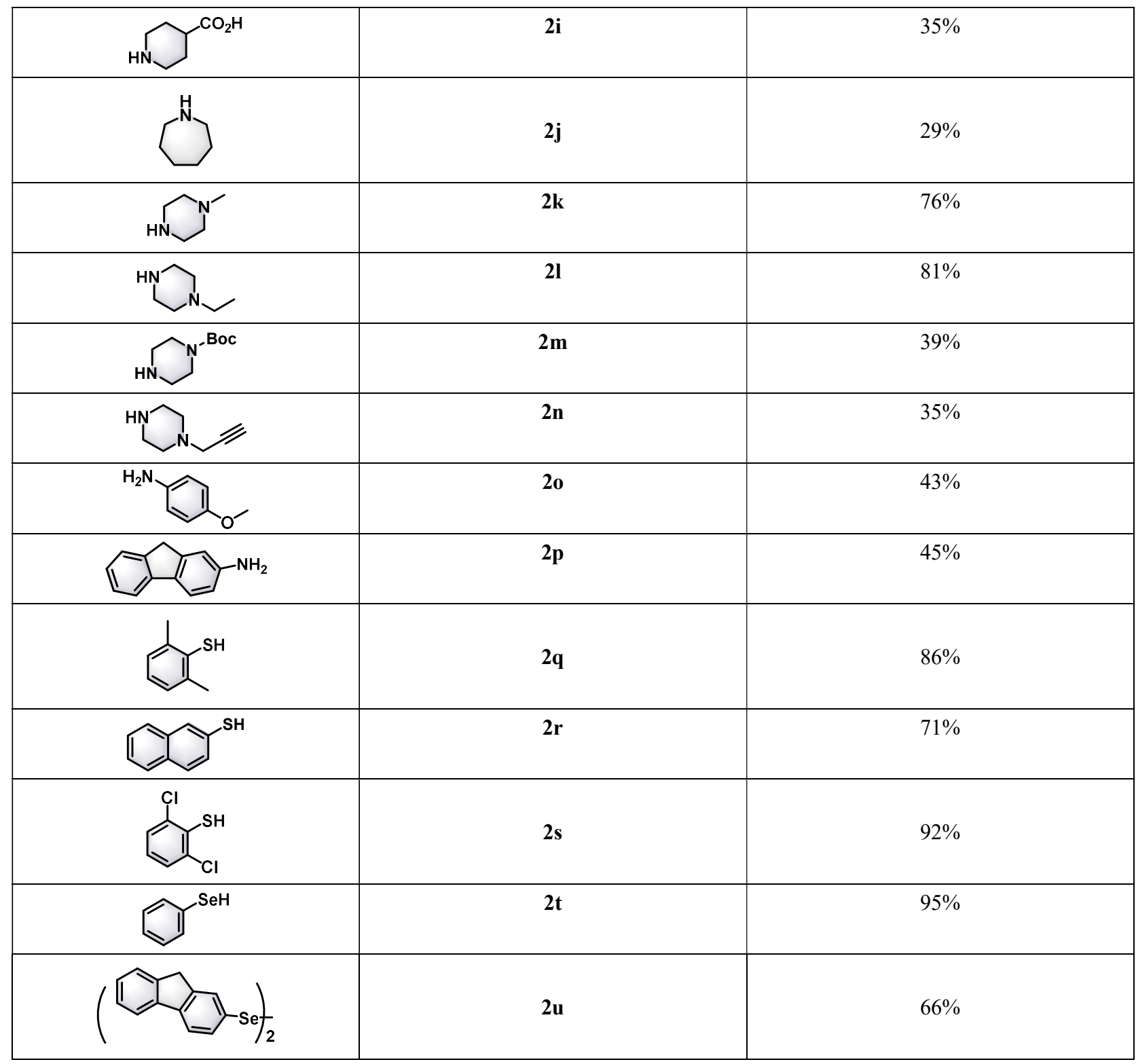

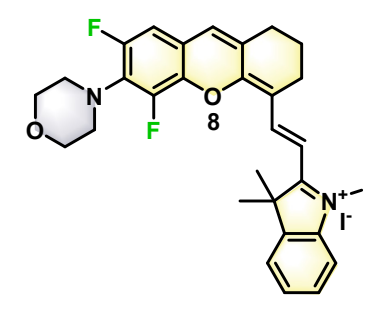

8: $61 \%$ yield; Dark green solid; ${ }^{1} \mathrm{H}$ NMR $\left(400.0 \mathrm{MHz}, \mathrm{DMSO}-d_{6}\right): \delta=8.54(\mathrm{~d}, J=15.4 \mathrm{~Hz}, 1 \mathrm{H}), 7.83(\mathrm{~d}, J=7.3 \mathrm{~Hz}$, 1H), $7.74(\mathrm{~d}, J=7.9 \mathrm{~Hz}, 1 \mathrm{H}), 7.57$ (dd, $J=7.3,7.5 \mathrm{~Hz}, 1 \mathrm{H}), 7.50$ (t, $J=7.4 \mathrm{~Hz}, 1 \mathrm{H}), 7.26(\mathrm{~m}, 2 \mathrm{H}), 6.71$ (d, $J=15.4 \mathrm{~Hz}$, 1H), 3.95 (s, 3H), 3.73 (t, $J=4.2 \mathrm{~Hz}, 4 \mathrm{H}), 3.26$ (brs, 4H), $2.70(\mathrm{~m}, 4 \mathrm{H}), 1.82(\mathrm{~m}, 2 \mathrm{H}), 1.74(\mathrm{~s}, 6 \mathrm{H}) \mathrm{ppm} ;{ }^{19} \mathrm{~F}$ NMR $(376.5$ MHz, DMSO- $\left.d_{6}\right): \delta=-124.3(\mathrm{~m}, 1 \mathrm{~F}),-146.5(\mathrm{~s}, 1 \mathrm{~F}) \mathrm{ppm} ;{ }^{13} \mathrm{C}$ NMR $\left(100.0 \mathrm{MHz}, \mathrm{DMSO}-d_{6}\right): \delta=179.2,157.9,153.3$ $\left(\mathrm{dd}, J_{\mathrm{C}-\mathrm{F}}=5.9 \mathrm{~Hz},{ }^{1} J_{\mathrm{C}-\mathrm{F}}=243.8 \mathrm{~Hz}\right), 144.3\left(\mathrm{dd}, J_{\mathrm{C}-\mathrm{F}}=7,6 \mathrm{~Hz},{ }^{1} J_{\mathrm{C}-\mathrm{F}}=247.9 \mathrm{~Hz}\right), 145.2,142.8,142.6,138.6\left(\mathrm{dd}, J_{\mathrm{C}-\mathrm{F}}=\right.$ $\left.1.6 \mathrm{~Hz}, J_{\mathrm{C}-\mathrm{F}}=10.1 \mathrm{~Hz}\right), 130.3\left(\mathrm{dd}, J_{\mathrm{C}-\mathrm{F}}=9.0 \mathrm{~Hz}, J_{\mathrm{C}-\mathrm{F}}=14,5 \mathrm{~Hz}\right), 130.0,129.7,129.4,128.3,123.3,117.0\left(\mathrm{~d}, J_{\mathrm{C}-\mathrm{F}}=11.0\right.$ 
$\mathrm{Hz}), 115.0,114.3,109.1\left(\mathrm{dd}, J_{\mathrm{C}-\mathrm{F}}=2.2 \mathrm{~Hz}, J_{\mathrm{C}-\mathrm{F}}=24.3 \mathrm{~Hz}\right), 107.6,67.1,51.4,51.2,33.7,29.1,27.4,23.9,20.2 \mathrm{ppm}$; HRMS (ESI+): $\mathrm{m} / \mathrm{z}$ calcd for $\mathrm{C}_{30} \mathrm{H}_{31} \mathrm{~F}_{2} \mathrm{~N}_{2} \mathrm{O}_{2}{ }^{+}[\mathrm{M}]^{+0}$ 489.2348, found 489.2343; HPLC (system A): $t_{\mathrm{R}}=4.8$ min (purity $100 \%$ at $600 \mathrm{~nm}$ ); LRMS (ESI+, recorded during RP-HPLC analysis): $m / z 489.2[\mathrm{M}]^{+{ }^{\circ}}$ (100), calcd for $\mathrm{C}_{30} \mathrm{H}_{31} \mathrm{~F}_{2} \mathrm{~N}_{2} \mathrm{O}_{2}{ }^{+}$ 489.2; UV-vis (recorded during the HPLC analysis): $\lambda_{\max }=602 \mathrm{~nm}$ (broad peak).

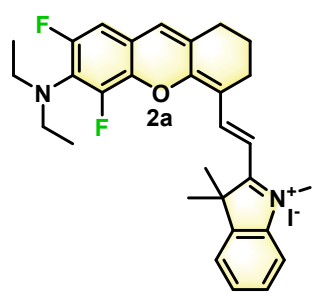

2a: $25 \%$ yield; Dark green solid; ${ }^{1} \mathrm{H}$ NMR $\left(400.0 \mathrm{MHz}, \mathrm{DMSO}-d_{6}\right): \delta=8.58(\mathrm{~d}, J=15.3 \mathrm{~Hz}, 1 \mathrm{H}), 7.82(\mathrm{~d}, J=7.3 \mathrm{~Hz}$, 1H), 7.73 (d, $J=7.9 \mathrm{~Hz}, 1 \mathrm{H}), 7.57$ (dd, $J=7.5,7.7 \mathrm{~Hz}, 1 \mathrm{H}), 7.50$ (t, $J=7.4 \mathrm{~Hz}, 1 \mathrm{H}), 7.26(\mathrm{~m}, 2 \mathrm{H}), 6.70$ (d, $J=15.3 \mathrm{~Hz}$, $1 \mathrm{H}), 3.94(\mathrm{~s}, 3 \mathrm{H}), 3.26(\mathrm{q}, J=7.0 \mathrm{~Hz}, 4 \mathrm{H}), 2.70(\mathrm{~m}, 4 \mathrm{H}), 1.82(\mathrm{~m}, 2 \mathrm{H}), 1.74(\mathrm{~s}, 6 \mathrm{H}), 1.05(\mathrm{t}, J=7.0 \mathrm{~Hz}, 6 \mathrm{H}) \mathrm{ppm} ;{ }^{19} \mathrm{~F}$ NMR (376.5 MHz, DMSO- $\left.d_{6}\right): \delta=-123.2(\mathrm{~m}, 1 \mathrm{~F}),-145.0(\mathrm{~s}, 1 \mathrm{~F}) \mathrm{ppm} ;{ }^{13} \mathrm{C}$ NMR $\left(100.0 \mathrm{MHz}, \mathrm{DMSO}-d_{6}\right): \delta=178.7$, $157.5,154.4\left(\mathrm{dd}, J_{\mathrm{C}-\mathrm{F}}=5.8 \mathrm{~Hz},{ }^{1} J_{\mathrm{C}-\mathrm{F}}=244.3 \mathrm{~Hz}\right), 145.4\left(\mathrm{dd}, J_{\mathrm{C}-\mathrm{F}}=9.2 \mathrm{~Hz},{ }^{1} J_{\mathrm{C}-\mathrm{F}}=248.1 \mathrm{~Hz}\right), 144.8,142.3,142.1,138.0$ $\left(\mathrm{dd}, J_{\mathrm{C}-\mathrm{F}}=1.3 \mathrm{~Hz}, J_{\mathrm{C}-\mathrm{F}}=10.5 \mathrm{~Hz}\right), 129.6,129.4,128.9-128.6(\mathrm{~m}), 128.8,127.7,122.7,116.8\left(\mathrm{~d}, J_{\mathrm{C}-\mathrm{F}}=11.6 \mathrm{~Hz}\right), 114.4$, $113.7,108.3\left(\mathrm{dd}, J_{\mathrm{C}-\mathrm{F}}=2.0 \mathrm{~Hz}, J_{\mathrm{C}-\mathrm{F}}=24.5 \mathrm{~Hz}\right), 106.9,50.7,46.4,33.1,28.6,26.9,23.4,19.8,13.2 \mathrm{ppm} ; \mathrm{HRMS}(\mathrm{ESI}+$ ): $\mathrm{m} / \mathrm{z}$ calcd for $\mathrm{C}_{30} \mathrm{H}_{33} \mathrm{~F}_{2} \mathrm{~N}_{2} \mathrm{O}^{+}[\mathrm{M}]^{+\circ} 475.2555$, found 475.2552; HPLC (system A): $t_{\mathrm{R}}=5.4 \min$ (purity $99 \%$ at $600 \mathrm{~nm}$ ); LRMS (ESI+, recorded during RP-HPLC analysis): $m / z 474.9[\mathrm{M}]^{+^{\circ}}$ (100), calcd for $\mathrm{C}_{30} \mathrm{H}_{33} \mathrm{~F}_{2} \mathrm{~N}_{2} \mathrm{O}^{+} 475.3$; UV-vis (recorded during the HPLC analysis): $\lambda_{\max }=598 \mathrm{~nm}$ (broad peak).

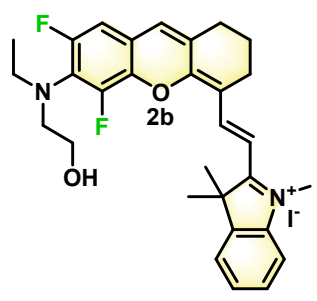

2b: $34 \%$ yield; Dark green solid; ${ }^{1} \mathrm{H}$ NMR $\left(400.0 \mathrm{MHz}, \mathrm{CD}_{3} \mathrm{OD}\right): \delta=8.80(\mathrm{~d}, J=15.2 \mathrm{~Hz}, 1 \mathrm{H}), 7.69(\mathrm{~d}, J=7.3 \mathrm{~Hz}, 1 \mathrm{H})$, $7.62(\mathrm{~d}, J=7.9 \mathrm{~Hz}, 1 \mathrm{H}), 7.56(\mathrm{dd}, J=7.1,7.6 \mathrm{~Hz}, 1 \mathrm{H}), 7.50(\mathrm{t}, J=7.3 \mathrm{~Hz}, 1 \mathrm{H}), 7.21(\mathrm{~s}, 1 \mathrm{H}), 7.06\left(\mathrm{dd}, J_{\mathrm{H}-\mathrm{F}}=1.1 \mathrm{~Hz}, J_{\mathrm{H}-}\right.$ $\mathrm{F}=11.6 \mathrm{~Hz}, 1 \mathrm{H}), 6.66(\mathrm{~d}, J=15.2 \mathrm{~Hz}, 1 \mathrm{H}), 3.93(\mathrm{~s}, 3 \mathrm{H}), 3.68(\mathrm{t}, J=6.0 \mathrm{~Hz}, 2 \mathrm{H}), 3.42(\mathrm{~m}, 4 \mathrm{H}), 2.76(\mathrm{~m}, 4 \mathrm{H}), 1.94(\mathrm{~m}$, $2 \mathrm{H}), 1.83(\mathrm{~s}, 6 \mathrm{H}), 1.14(\mathrm{t}, J=7.1 \mathrm{~Hz}, 3 \mathrm{H}) \mathrm{ppm} ;{ }^{19} \mathrm{~F} \mathrm{NMR}\left(376.5 \mathrm{MHz}, \mathrm{CD}_{3} \mathrm{OD}\right): \delta=-123.7(\mathrm{~m}, 1 \mathrm{~F}),-145.3(\mathrm{~s}, 1 \mathrm{~F}) \mathrm{ppm}$; ${ }^{13} \mathrm{C} \mathrm{NMR}\left(100.0 \mathrm{MHz}, \mathrm{CD}_{3} \mathrm{OD}\right): \delta=180.8,160.5,156.7\left(\mathrm{dd}, J_{\mathrm{C}-\mathrm{F}}=5.9 \mathrm{~Hz},{ }^{1} J_{\mathrm{C}-\mathrm{F}}=245.3 \mathrm{~Hz}\right), 147.6,147.4\left(\mathrm{dd}, J_{\mathrm{C}-\mathrm{F}}=\right.$ $\left.8.0 \mathrm{~Hz},{ }^{1} J_{\mathrm{C}-\mathrm{F}}=248.2 \mathrm{~Hz}\right), 143.8,143.7,140.1\left(\mathrm{dd}, J_{\mathrm{C}-\mathrm{F}}=2.1 \mathrm{~Hz}, J_{\mathrm{C}-\mathrm{F}}=10.9 \mathrm{~Hz}\right), 131.7\left(\mathrm{t}, J_{\mathrm{C}-\mathrm{F}}=2.9 \mathrm{~Hz}\right), 131.5\left(\mathrm{dd}, J_{\mathrm{C}-\mathrm{F}}\right.$ $\left.=10.1 \mathrm{~Hz}, J_{\mathrm{C}-\mathrm{F}}=15.6 \mathrm{~Hz}\right), 131.3,130.4,129.2,123.9,118.6\left(\mathrm{~d}, J_{\mathrm{C}-\mathrm{F}}=11.3 \mathrm{~Hz}\right), 116.4,114.4,109.4\left(\mathrm{dd}, J_{\mathrm{C}-\mathrm{F}}=3.1 \mathrm{~Hz}\right.$, $\left.J_{\mathrm{C}-\mathrm{F}}=24.9 \mathrm{~Hz}\right), 107.2,61.4,55.9\left(\mathrm{t}, J_{\mathrm{C}-\mathrm{F}}=3.3 \mathrm{~Hz}\right), 52.5,33.4,30.4,28.1,25.1,21.7,14.0 \mathrm{ppm} ; \mathrm{HRMS}$ (ESI+): $\mathrm{m} / \mathrm{z}$ calcd for $\mathrm{C}_{30} \mathrm{H}_{33} \mathrm{~F}_{2} \mathrm{~N}_{2} \mathrm{O}_{2}{ }^{+}[\mathrm{M}]^{+\circ} 491.2505$, found 491.2488; HPLC (system A): $t_{\mathrm{R}}=4.7$ min (purity $>99 \%$ at $600 \mathrm{~nm}$ ); LRMS (ESI+, recorded during RP-HPLC analysis): $m / z 491.0[\mathrm{M}]^{+{ }^{\circ}}$ (100), calcd for $\mathrm{C}_{30} \mathrm{H}_{33} \mathrm{~F}_{2} \mathrm{~N}_{2} \mathrm{O}_{2}{ }^{+}$491.2; UV-vis (recorded during the HPLC analysis): $\lambda_{\max }=598 \mathrm{~nm}$ (broad peak). 


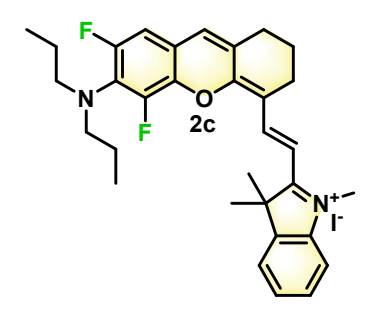

2c: $16 \%$ yield; Dark green solid; ${ }^{1} \mathrm{H}$ NMR $\left(400.0 \mathrm{MHz}, \mathrm{CD}_{3} \mathrm{OD}\right): \delta=8.78(\mathrm{~d}, J=15.1 \mathrm{~Hz}, 1 \mathrm{H}), 7.69(\mathrm{~d}, J=7.3 \mathrm{~Hz}, 1 \mathrm{H})$, $7.62(\mathrm{~d}, J=7.9 \mathrm{~Hz}, 1 \mathrm{H}), 7.56(\mathrm{dd}, J=7.4,7.8 \mathrm{~Hz}, 1 \mathrm{H}), 7.49(\mathrm{t}, J=7.4 \mathrm{~Hz}, 1 \mathrm{H}), 7.22(\mathrm{~s}, 1 \mathrm{H}), 7.06\left(\mathrm{dd}, J_{\mathrm{H}-\mathrm{F}}=1.0 \mathrm{~Hz}, J_{\mathrm{H}-}\right.$ F=11.6 Hz, 1H), $6.66(\mathrm{~d}, J=15.2 \mathrm{~Hz}, 1 \mathrm{H}), 3.94(\mathrm{~s}, 3 \mathrm{H}), 3.27(\mathrm{t}, J=7.0 \mathrm{~Hz}, 4 \mathrm{H}), 2.75(\mathrm{~m}, 4 \mathrm{H}), 1.94(\mathrm{brs}, 2 \mathrm{H}), 1.83(\mathrm{~s}$, $6 \mathrm{H}), 1.56(\mathrm{~m}, 4 \mathrm{H}), 0.91$ (t, $\left.J=7.3 \mathrm{~Hz}, 6 \mathrm{H}) \mathrm{ppm} ;{ }^{19} \mathrm{~F} \mathrm{NMR} \mathrm{(376.5} \mathrm{MHz,} \mathrm{CD}_{3} \mathrm{OD}\right): \delta=-123.8(\mathrm{~m}, 1 \mathrm{~F}),-145.9$ (s, $\left.1 \mathrm{~F}\right) \mathrm{ppm}$; ${ }^{13} \mathrm{C} \mathrm{NMR}\left(100.0 \mathrm{MHz}, \mathrm{CD}_{3} \mathrm{OD}\right): \delta=180.7,160.5,156.5\left(\mathrm{dd}, J_{\mathrm{C}-\mathrm{F}}=6.0 \mathrm{~Hz},{ }^{1} J_{\mathrm{C}-\mathrm{F}}=245.2 \mathrm{~Hz}\right), 147.5,147.1\left(\mathrm{dd}, J_{\mathrm{C}-\mathrm{F}}=\right.$ $\left.7.9 \mathrm{~Hz},{ }^{1} J_{\mathrm{C}-\mathrm{F}}=247.8 \mathrm{~Hz}\right), 143.8,143.7,140.1\left(\mathrm{dd}, J_{\mathrm{C}-\mathrm{F}}=1.7 \mathrm{~Hz}, J_{\mathrm{C}-\mathrm{F}}=10.6 \mathrm{~Hz}\right), 131.8(\mathrm{~m}), 131.8,130.4,129.1,123.9$, $118.2\left(\mathrm{~d}, J_{\mathrm{C}-\mathrm{F}}=1.3 \mathrm{~Hz}\right), 116.4,114.5,109.5\left(\mathrm{~d}, J_{\mathrm{C}-\mathrm{F}}=24.6 \mathrm{~Hz}\right), 107.2,56.1\left(\mathrm{t}, J_{\mathrm{C}-\mathrm{F}}=3.3 \mathrm{~Hz}\right), 52.4,33.7,30.4,28.1,25.1$, 22.7, 21.6, 11.8 ppm; HRMS (ESI+): $\mathrm{m} / \mathrm{z}$ calcd for $\mathrm{C}_{32} \mathrm{H}_{37} \mathrm{~F}_{2} \mathrm{~N}_{2} \mathrm{O}^{+}[\mathrm{M}]^{+}$503.2868, found 503.2868; HPLC (system A): $t_{\mathrm{R}}=5.7 \mathrm{~min}$ (purity $>99 \%$ at $600 \mathrm{~nm}$ ); LRMS (ESI+, recorded during RP-HPLC analysis): $\mathrm{m} / z 503.1[\mathrm{M}]^{+^{\circ}}(100)$, calcd for $\mathrm{C}_{32} \mathrm{H}_{37} \mathrm{~F}_{2} \mathrm{~N}_{2} \mathrm{O}^{+} 503.3$; UV-vis (recorded during the HPLC analysis): $\lambda_{\max }=615 \mathrm{~nm}$ (broad peak).

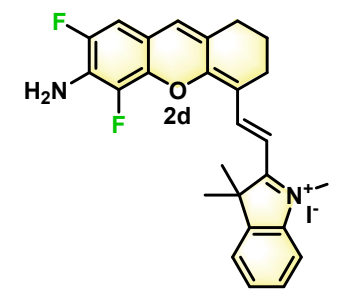

2d: $44 \%$ yield; Dark green solid; ${ }^{1} \mathrm{H}$ NMR $\left(600.0 \mathrm{MHz}, \mathrm{DMSO}-d_{6}\right): \delta=8.58(\mathrm{~d}, J=14.9 \mathrm{~Hz}, 1 \mathrm{H}), 7.76(\mathrm{~d}, J=7.4 \mathrm{~Hz}$, $1 \mathrm{H}), 7.64(\mathrm{~d}, J=8.0 \mathrm{~Hz}, 1 \mathrm{H}), 7.53(\mathrm{dd}, J=7.5,7.9 \mathrm{~Hz}, 1 \mathrm{H}), 7.43(\mathrm{t}, J=7.5 \mathrm{~Hz}, 1 \mathrm{H}), 7.41(\mathrm{~s}, 1 \mathrm{H}), 7.26\left(\mathrm{~d}, J_{\mathrm{H}-\mathrm{F}}=10.5\right.$ $\mathrm{Hz}, 1 \mathrm{H}), 6.60$ (brs, 2H), 6.54 (d, $J=15.0 \mathrm{~Hz}, 1 \mathrm{H}), 3.84$ (s, 3H), 2.71 (t, $J=5.8 \mathrm{~Hz}, 2 \mathrm{H}), 2.67$ (t, $J=6.0 \mathrm{~Hz}, 2 \mathrm{H}), 1.82$ $(\mathrm{m}, 2 \mathrm{H}), 1.72(\mathrm{~s}, 6 \mathrm{H}) \mathrm{ppm} ;{ }^{19} \mathrm{~F}$ NMR $\left(376.5 \mathrm{MHz}, \mathrm{DMSO}-d_{6}\right): \delta=-133.2(\mathrm{~m}, 1 \mathrm{~F}),-157.4\left(\mathrm{~d}, J_{\mathrm{F}-\mathrm{F}}=11.4 \mathrm{~Hz}, 1 \mathrm{~F}\right) \mathrm{ppm}$; ${ }^{13} \mathrm{C}$ NMR $\left(100.0 \mathrm{MHz}, \mathrm{DMSO}-d_{6}\right): \delta=177.0,159.4,147.6\left(\mathrm{dd}, J_{\mathrm{C}-\mathrm{F}}=7.4 \mathrm{~Hz},{ }^{1} J_{\mathrm{C}-\mathrm{F}}=239.5 \mathrm{~Hz}\right), 144.0,142.3,141.7$, $138.6\left(\mathrm{~d}, J_{\mathrm{C}-\mathrm{F}}=8.0 \mathrm{~Hz}\right), 137.1\left(\mathrm{dd}, J_{\mathrm{C}-\mathrm{F}}=8.7 \mathrm{~Hz},{ }^{1} J_{\mathrm{C}-\mathrm{F}}=240.4 \mathrm{~Hz}\right), 133.0,130.4\left(\mathrm{dd}, J_{\mathrm{C}-\mathrm{F}}=12.7 \mathrm{~Hz}, J_{\mathrm{C}-\mathrm{F}}=18.5 \mathrm{~Hz}\right)$, 128.7, 126.7, 125.4, 122.6, 113.9, 112.9, 109.6 (d, $\left.J_{\mathrm{C}-\mathrm{F}}=10.2 \mathrm{~Hz}\right), 107.8\left(\mathrm{~d}, J_{\mathrm{C}-\mathrm{F}}=20.9 \mathrm{~Hz}\right), 104.3,50.0,32.4,28.2,27.2$, 23.4, 19.9 ppm; HRMS (ESI+): m/z calcd for $\mathrm{C}_{26} \mathrm{H}_{25} \mathrm{~F}_{2} \mathrm{~N}_{2} \mathrm{O}^{+}[\mathrm{M}]^{+0} 419.1929$, found 419.1916; HPLC (system A): $t_{\mathrm{R}}=$ $4.6 \mathrm{~min}$ (purity $99 \%$ at $600 \mathrm{~nm}$ ); LRMS (ESI+, recorded during RP-HPLC analysis): $\mathrm{m} / z 419.0[\mathrm{M}]^{+^{\circ}}$ (100), calcd for $\mathrm{C}_{26} \mathrm{H}_{25} \mathrm{~F}_{2} \mathrm{~N}_{2} \mathrm{O}^{+} 419.2$; UV-vis (recorded during the HPLC analysis): $\lambda_{\max }=620$ and $671 \mathrm{~nm}$ (broad peak).

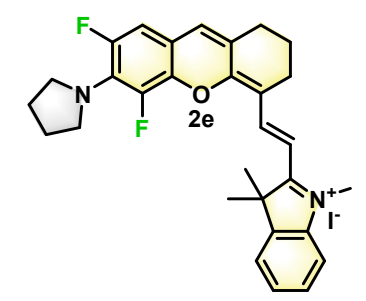

2e: $61 \%$ yield; Dark green solid; ${ }^{1} \mathrm{H}$ NMR $\left(400.0 \mathrm{MHz}, \mathrm{CD}_{3} \mathrm{OD}\right): \delta=8.72(\mathrm{~d}, J=14.9 \mathrm{~Hz}, 1 \mathrm{H}), 7.64(\mathrm{~d}, J=7.4 \mathrm{~Hz}, 1 \mathrm{H})$, $7.52(\mathrm{~m}, 2 \mathrm{H}), 7.43(\mathrm{~m}, 1 \mathrm{H}), 7.27(\mathrm{~s}, 1 \mathrm{H}), 7.02\left(\mathrm{~d}, J_{\mathrm{H}-\mathrm{F}}=13.6 \mathrm{~Hz}, 1 \mathrm{H}\right), 6.50(\mathrm{~d}, J=14.9 \mathrm{~Hz}, 1 \mathrm{H}), 3.84(\mathrm{~s}, 3 \mathrm{H}), 3.74$ (brs, 4H), $2.74(\mathrm{~m}, 4 \mathrm{H}), 1.95(\mathrm{~m}, 6 \mathrm{H}), 1.79(\mathrm{~s}, 6 \mathrm{H}) \mathrm{ppm} ;{ }^{19} \mathrm{~F}$ NMR (376.5 MHz, CD $\left.{ }_{3} \mathrm{OD}\right): \delta=-126.0(\mathrm{~m}, 1 \mathrm{~F}),-153.1(\mathrm{~s}, 1 \mathrm{~F})$ ppm; ${ }^{13} \mathrm{C}$ NMR $\left(100.0 \mathrm{MHz}, \mathrm{CD}_{3} \mathrm{OD}\right): \delta=179.3,162.0,151.6\left(\mathrm{dd}, J_{\mathrm{C}-\mathrm{F}}=8.1 \mathrm{~Hz},{ }^{1} J_{\mathrm{C}-\mathrm{F}}=243.5 \mathrm{~Hz}\right), 146.6,143.9,143.4$, $141.4\left(\mathrm{~d}, J_{\mathrm{C}-\mathrm{F}}=11.0 \mathrm{~Hz}\right), 141.3\left(\mathrm{dd}, J_{\mathrm{C}-\mathrm{F}}=9.4 \mathrm{~Hz},{ }^{1} J_{\mathrm{C}-\mathrm{F}}=243.2 \mathrm{~Hz}\right), 134.2,131.9\left(\mathrm{dd}, J_{\mathrm{C}-\mathrm{F}}=8.3 \mathrm{~Hz}, J_{\mathrm{C}-\mathrm{F}}=14.4 \mathrm{~Hz}\right)$, 
$130.3,128.4,128.1,123.8,116.2,113.7,113.4\left(\mathrm{~d}, J_{\mathrm{C}-\mathrm{F}}=11.3 \mathrm{~Hz}\right), 110.0\left(\mathrm{~d}, J_{\mathrm{C}-\mathrm{F}}=25.3 \mathrm{~Hz}\right), 105.1,53.1\left(\mathrm{t}, J_{\mathrm{C}-\mathrm{F}}=6.4 \mathrm{~Hz}\right)$, 51.9, 33.0, 30.9, 30.1, 28.4, 26.9, 21.8 ppm; HRMS (ESI+): $\mathrm{m} / \mathrm{z}$ calcd for $\mathrm{C}_{30} \mathrm{H}_{31} \mathrm{~F}_{2} \mathrm{~N}_{2} \mathrm{O}^{+}[\mathrm{M}]^{+0}$ 473.2399, found 473.2386; HPLC (system A): $t_{\mathrm{R}}=5.5 \mathrm{~min}$ (purity $98 \%$ at $600 \mathrm{~nm}$ ); LRMS (ESI+, recorded during RP-HPLC analysis): $\mathrm{m} / z 473.2$ $[\mathrm{M}]^{+\circ}(100)$, calcd for $\mathrm{C}_{30} \mathrm{H}_{31} \mathrm{~F}_{2} \mathrm{~N}_{2} \mathrm{O}^{+} 473.2$; UV-vis (recorded during the HPLC analysis): $\lambda_{\max }=647$ and $701 \mathrm{~nm}$ (broad peak).

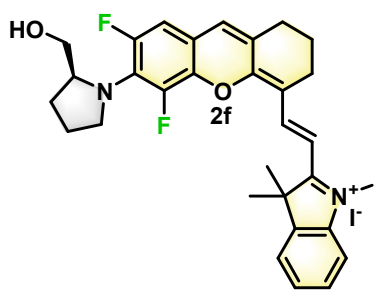

2f: $10 \%$ yield; Dark blue solid; Purification using semi-preparative RP-HPLC; ${ }^{1} \mathrm{H}$ NMR (400.0 MHz, $\left.\mathrm{CD}_{3} \mathrm{OD}\right): \delta=8.79$ $(\mathrm{d}, J=15.0 \mathrm{~Hz}, 1 \mathrm{H}), 7.65(\mathrm{~d}, J=7.4 \mathrm{~Hz}, 1 \mathrm{H}), 7.59-7.48(\mathrm{~m}, 2 \mathrm{H}), 7.48-7.43(\mathrm{~m}, 1 \mathrm{H}), 7.26(\mathrm{~d}, J=1.6 \mathrm{~Hz}, 1 \mathrm{H}), 7.05(\mathrm{dd}$, $J=13.0,2.0 \mathrm{~Hz}, 1 \mathrm{H}), 6.55(\mathrm{~d}, J=15.0 \mathrm{~Hz}, 1 \mathrm{H}), 4.38(\mathrm{~m}, J=7.5 \mathrm{~Hz}, 1 \mathrm{H}), 4.00-3.90(\mathrm{~m}, 1 \mathrm{H}), 3.86(\mathrm{~s}, 3 \mathrm{H}), 3.65-3.42$ (m, 3H), 2.75 (dt, $J=20.5,6.4 \mathrm{~Hz}, 4 \mathrm{H}), 2.23-2.17(\mathrm{~m}, J=7.3,3.9 \mathrm{~Hz}, 1 \mathrm{H}), 2.10-2.02(\mathrm{~m}, 1 \mathrm{H}), 1.98-1.88(\mathrm{~m}, J=12.5$, $4.9 \mathrm{~Hz}, 4 \mathrm{H}), 1.81(\mathrm{~d}, J=3.2 \mathrm{~Hz}, 6 \mathrm{H}) ;{ }^{13} \mathrm{C} \mathrm{NMR}\left(100.0 \mathrm{MHz}, \mathrm{CD}_{3} \mathrm{OD}\right): \delta=179.8,161.5,147.0,143.7,143.4,133.3$, 130.2, 129.0, 128.5, 123.6, 116.0, 115.1, 115.0, 113.8, 109.6, 109.4, 105.5, 64.7, 63.0, 54.2, 52.0, 32.8, 30.1, 29.5, 28.1, 26.0, 24.9, 21.6 pm; IR (film) $v_{\max }=3386,2925,2861,1686,1636,1600,1565,1536,1503,1459,1402,1366,1307$, 1292, 1270, 1237, 1202, 1171, 1114, 1065, 1043, 1020, 929, $837 \mathrm{~cm}^{-1}$; HRMS (ESI + ): $m / z$ calcd for $\mathrm{C}_{31} \mathrm{H}_{33} \mathrm{~F}_{2} \mathrm{~N}_{2} \mathrm{O}_{2}{ }^{+}[\mathrm{M}]^{+\circ}$ 503.2505, found 503.2500; HPLC (system A): $t_{\mathrm{R}}=4.8 \mathrm{~min}$ (purity $98 \%$ at $600 \mathrm{~nm}$ ); LRMS (ESI+, recorded during RPHPLC analysis): $m / z 503.3[\mathrm{M}]^{+\circ}$ (100), calcd for $\mathrm{C}_{31} \mathrm{H}_{33} \mathrm{~F}_{2} \mathrm{~N}_{2} \mathrm{O}_{2}{ }^{+}$503.3; UV-vis (recorded during the HPLC analysis): $\lambda_{\max }=637$ and $691 \mathrm{~nm}$ (broad peak).

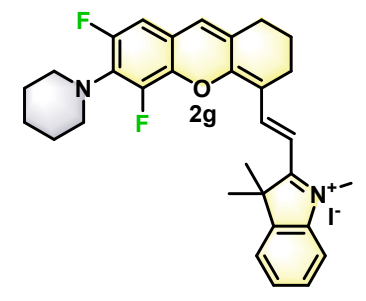

2g: $65 \%$ yield; Dark green solid; ${ }^{1} \mathrm{H}$ NMR $\left(400.0 \mathrm{MHz}, \mathrm{CDCl}_{3}\right): \delta=8.69(\mathrm{~d}, J=15.1 \mathrm{~Hz}, 1 \mathrm{H}), 7.46(\mathrm{~m}, 4 \mathrm{H}) 6.97(\mathrm{~s}, 1 \mathrm{H})$, $6.82(\mathrm{~m}, 2 \mathrm{H}), 4.16$ (s, 3H), 3.31 (brs, 4H), 2.90 (t, $J=6.0 \mathrm{~Hz}, 2 \mathrm{H}), 2.72(\mathrm{t}, J=5.4 \mathrm{~Hz}, 2 \mathrm{H}), 1.96(\mathrm{~m}, 2 \mathrm{H}), 1.81(\mathrm{~s}, 6 \mathrm{H})$, $1.72(\mathrm{~m}, 4 \mathrm{H}), 1.66(\mathrm{~m}, 2 \mathrm{H}) \mathrm{ppm} ;{ }^{19} \mathrm{~F}$ NMR $\left(376.5 \mathrm{MHz}, \mathrm{CD}_{3} \mathrm{OD}\right): \delta=-125.1\left(\mathrm{~d}, J_{\mathrm{H}-\mathrm{F}}=6.4 \mathrm{~Hz}, 1 \mathrm{~F}\right),-147.1(\mathrm{~s}, 1 \mathrm{~F}) \mathrm{ppm}$; ${ }^{13} \mathrm{C}$ NMR $\left(100.0 \mathrm{MHz}, \mathrm{CD}_{3} \mathrm{OD}\right): \delta=180.7,160.7,155.3\left(\mathrm{dd}, J_{\mathrm{C}-\mathrm{F}}=6.8 \mathrm{~Hz},{ }^{1} J_{\mathrm{C}-\mathrm{F}}=245.0 \mathrm{~Hz}\right), 147.5,145.8\left(\mathrm{dd}, J_{\mathrm{C}-\mathrm{F}}=\right.$ $\left.7.0 \mathrm{~Hz},{ }^{1} J_{\mathrm{C}-\mathrm{F}}=247.2 \mathrm{~Hz}\right), 143.8,143.7,140.4(\mathrm{~m}), 133.6\left(\mathrm{dd}, J_{\mathrm{C}-\mathrm{F}}=9.1 \mathrm{~Hz}, J_{\mathrm{C}-\mathrm{F}}=14.7 \mathrm{~Hz}\right), 132.0,130.8,130.4,129.1$, $123.9,117.6\left(\mathrm{~d}, J_{\mathrm{C}-\mathrm{F}}=11.0 \mathrm{~Hz}\right), 116.4,114.4,109.6\left(\mathrm{dd}, J_{\mathrm{C}-\mathrm{F}}=2.8 \mathrm{~Hz}, J_{\mathrm{C}-\mathrm{F}}=24.4 \mathrm{~Hz}\right), 106.9,53.7\left(\mathrm{t}, J_{\mathrm{C}-\mathrm{F}}=3.8 \mathrm{~Hz}\right), 52.4$, 33.4, 30.3, 28.1, 27.9, 25.4, 25.1, 21.7 ppm; HRMS (ESI+): $\mathrm{m} / \mathrm{z}$ calcd for $\mathrm{C}_{31} \mathrm{H}_{33} \mathrm{~F}_{2} \mathrm{~N}_{2} \mathrm{O}^{+}[\mathrm{M}]^{+}$487.2555, found 487.2524; HPLC (system A): $t_{\mathrm{R}}=5.5 \mathrm{~min}$ (purity $100 \%$ at $600 \mathrm{~nm}$ ); LRMS (ESI+, recorded during RP-HPLC analysis): $\mathrm{m} / z 487.2$ $[\mathrm{M}]^{+\circ}(100)$, calcd for $\mathrm{C}_{31} \mathrm{H}_{33} \mathrm{~F}_{2} \mathrm{~N}_{2} \mathrm{O}^{+} 487.3$; UV-vis (recorded during the HPLC analysis): $\lambda_{\max }=613 \mathrm{~nm}$ (broad peak). 


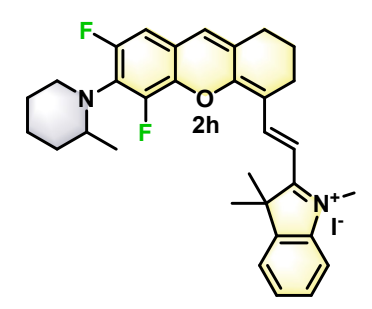

2h: 8\% yield; Dark green solid; ${ }^{1} \mathrm{H}$ NMR (400.0 MHz, $\left.\mathrm{CD}_{3} \mathrm{OD}\right): \delta=8.80(\mathrm{~d}, J=15.2 \mathrm{~Hz}, 1 \mathrm{H}), 7.70(\mathrm{~d}, J=7.3 \mathrm{~Hz}, 1 \mathrm{H})$, $7.64(\mathrm{~d}, J=7.9 \mathrm{~Hz}, 1 \mathrm{H}), 7.57(\mathrm{dd}, J=7.3,7.9 \mathrm{~Hz}, 1 \mathrm{H}), 7.51(\mathrm{t}, J=7.5 \mathrm{~Hz}, 1 \mathrm{H}), 7.19(\mathrm{~s}, 1 \mathrm{H}), 7.06\left(\mathrm{~d}, J_{\mathrm{H}-\mathrm{F}}=11.0 \mathrm{~Hz}, 1 \mathrm{H}\right)$, $6.69(\mathrm{~d}, J=15.3 \mathrm{~Hz}, 1 \mathrm{H}), 3.95(\mathrm{~s}, 3 \mathrm{H}), 3.42(\mathrm{~m}, 1 \mathrm{H}), 3.26(\mathrm{~m}, 1 \mathrm{H}), 3.08(\mathrm{~m}, 1 \mathrm{H}), 2.76(\mathrm{~m}, 4 \mathrm{H}), 1.94(\mathrm{~m}, 2 \mathrm{H}), 1.84(\mathrm{~s}$, $7 \mathrm{H}), 1.70(\mathrm{~m}, 3 \mathrm{H}), 1.56(\mathrm{~m}, 2 \mathrm{H}), 1.04(\mathrm{~d}, J=6.3 \mathrm{~Hz}, 3 \mathrm{H}) \mathrm{ppm} ;{ }^{19} \mathrm{~F}$ NMR $\left(376.5 \mathrm{MHz}, \mathrm{CD}_{3} \mathrm{OD}\right): \delta=-123.7(\mathrm{~m}, 1 \mathrm{~F}),-$ 145.3 (s, 1F) ppm; ${ }^{13} \mathrm{C}$ NMR (100.0 MHz, $\left.\mathrm{CD}_{3} \mathrm{OD}\right): \delta=181.1,160.0,157.9\left(\mathrm{~d},{ }^{1} J_{\mathrm{C}-\mathrm{F}}=245.2 \mathrm{~Hz}\right), 148.7\left(\mathrm{~d},{ }^{1} J_{\mathrm{C}-\mathrm{F}}=251.5\right.$ $\mathrm{Hz}), 147.7,143.9,143.7,139.8\left(\mathrm{~d}, J_{\mathrm{C}-\mathrm{F}}=10.5 \mathrm{~Hz}\right), 132.3\left(\mathrm{dd}, J_{\mathrm{C}-\mathrm{F}}=16.6 \mathrm{~Hz}, J_{\mathrm{C}-\mathrm{F}}=27.1 \mathrm{~Hz}\right), 132.1,131.0,130.4,129.3$, $120.1\left(\mathrm{~d}, J_{\mathrm{C}-\mathrm{F}}=10.2 \mathrm{~Hz}\right) 116.6,114.7,109.4\left(\mathrm{~d}, J_{\mathrm{C}-\mathrm{F}}=25.3 \mathrm{~Hz}\right), 107.9,56.0,52.6,35.2,30.9,30.4,28.1,27.9,25.1,24.0$, 21.6, 19.5 ppm; HRMS (ESI+): $\mathrm{m} / \mathrm{z}$ calcd for $\mathrm{C}_{32} \mathrm{H}_{35} \mathrm{~F}_{2} \mathrm{~N}_{2} \mathrm{O}^{+}[\mathrm{M}]^{+}$501.2712, found 501.2707; HPLC (system A): $t_{\mathrm{R}}=$ $5.6 \mathrm{~min}$ (purity $>99 \%$ at $600 \mathrm{~nm}$ ); LRMS (ESI+, recorded during RP-HPLC analysis): $m / z 501.1[\mathrm{M}]^{+\circ}(100)$, calcd for $\mathrm{C}_{32} \mathrm{H}_{35} \mathrm{~F}_{2} \mathrm{~N}_{2} \mathrm{O}^{+} 501.3$; UV-vis (recorded during the HPLC analysis): $\lambda_{\max }=589 \mathrm{~nm}$ (broad peak).

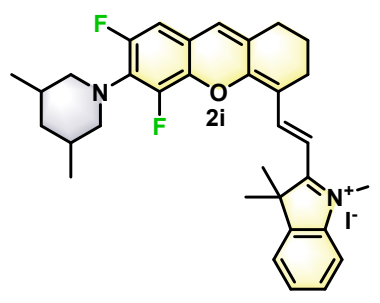

2i: $45 \%$ yield; Dark green solid; ${ }^{1} \mathrm{H}$ NMR (400.0 MHz, DMSO- $\left.d_{6}\right): \delta=8.52(\mathrm{~d}, J=15.3 \mathrm{~Hz}, 1 \mathrm{H}), 7.82(\mathrm{~d}, J=7.3 \mathrm{~Hz}$, $1 \mathrm{H}), 7.74(\mathrm{~d}, J=7.9 \mathrm{~Hz}, 1 \mathrm{H}), 7.56(\mathrm{dd}, J=7.1,7.5 \mathrm{~Hz}, 1 \mathrm{H}), 7.48(\mathrm{t}, J=7.5 \mathrm{~Hz}, 1 \mathrm{H}), 7.27(\mathrm{~s}, 1 \mathrm{H}), 7.22(\mathrm{~m}, 1 \mathrm{H}), 6.68(\mathrm{~d}$, $J=15.3 \mathrm{~Hz}, 1 \mathrm{H}), 3.94(\mathrm{~s}, 3 \mathrm{H}), 3.26(\mathrm{~d}, J=10.8 \mathrm{~Hz}, 2 \mathrm{H}), 2.64(\mathrm{~m}, 6 \mathrm{H}), 1.78(\mathrm{~m}, 12 \mathrm{H}), 0.86(\mathrm{~d}, J=6.5 \mathrm{~Hz}, 6 \mathrm{H}) \mathrm{ppm} ;{ }^{19} \mathrm{~F}$ NMR (376.5 MHz, DMSO- $\left.d_{6}\right): \delta=-124.2(\mathrm{~m}, 1 \mathrm{~F}),-146.9$ (s, $\left.1 \mathrm{~F}\right) \mathrm{ppm} ;{ }^{13} \mathrm{C}$ NMR $\left(100.0 \mathrm{MHz}, \mathrm{DMSO}-d_{6}\right): \delta=178.4$, $157.6,152.7\left(\mathrm{dd}, J_{\mathrm{C}-\mathrm{F}}=6.1 \mathrm{~Hz},{ }^{1} J_{\mathrm{C}-\mathrm{F}}=243.4 \mathrm{~Hz}\right), 144.5,143.6\left(\mathrm{dd}, J_{\mathrm{C}-\mathrm{F}}=7,5 \mathrm{~Hz},{ }^{1} J_{\mathrm{C}-\mathrm{F}}=247.1 \mathrm{~Hz}\right), 142.2,142.1,138.1$ $\left(\mathrm{dd}, J_{\mathrm{C}-\mathrm{F}}=1.5 \mathrm{~Hz}, J_{\mathrm{C}-\mathrm{F}}=10.2 \mathrm{~Hz}\right), 130.7\left(\mathrm{dd}, J_{\mathrm{C}-\mathrm{F}}=9.4 \mathrm{~Hz}, J_{\mathrm{C}-\mathrm{F}}=14.7 \mathrm{~Hz}\right), 129.6,129.0,128.8,127.6,122.7,115.7(\mathrm{~d}$, $\left.J_{\mathrm{C}-\mathrm{F}}=11.3 \mathrm{~Hz}\right), 114.4,113.7,108.4\left(\mathrm{dd}, J_{\mathrm{C}-\mathrm{F}}=1.7 \mathrm{~Hz}, J_{\mathrm{C}-\mathrm{F}}=24.3 \mathrm{~Hz}\right), 106.7,58.0,50.6,41.3,33.1,31.4,28.5,26.9$, 23.5, 19.7, 18.9 ppm; HRMS (ESI+): $\mathrm{m} / \mathrm{z}$ calcd for $\mathrm{C}_{33} \mathrm{H}_{37} \mathrm{~F}_{2} \mathrm{~N}_{2} \mathrm{O}^{+}[\mathrm{M}]^{+}$515.2868, found 515.2853; HPLC (system A): $t_{\mathrm{R}}=5.9 \mathrm{~min}$ (purity $98 \%$ at $600 \mathrm{~nm}$ ); LRMS (ESI+, recorded during RP-HPLC analysis): $m / z 514.9[\mathrm{M}]^{+^{\circ}}(100)$, calcd for $\mathrm{C}_{33} \mathrm{H}_{37} \mathrm{~F}_{2} \mathrm{~N}_{2} \mathrm{O}^{+} 515.3$; UV-vis (recorded during the HPLC analysis): $\lambda_{\max }=515 \mathrm{~nm}$ (broad peak).

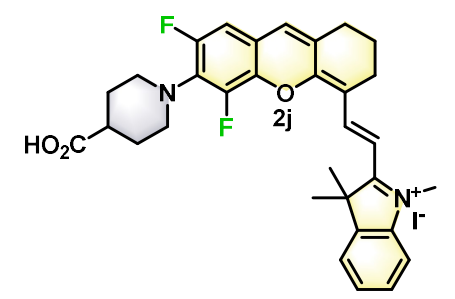

2j: 35\% yield; Dark green solid; ${ }^{1} \mathrm{H}$ NMR (400.0 MHz, $\left.\mathrm{CD}_{3} \mathrm{OD}\right): \delta=8.80(\mathrm{~d}, J=15.3 \mathrm{~Hz}, 1 \mathrm{H}), 7.69(\mathrm{~d}, J=7.3 \mathrm{~Hz}, 1 \mathrm{H})$, $7.62(\mathrm{~d}, J=7.8 \mathrm{~Hz}, 1 \mathrm{H}), 7.56(\mathrm{dd}, J=7.2,8.0 \mathrm{~Hz}, 1 \mathrm{H}), 7.50(\mathrm{t}, J=7.4 \mathrm{~Hz}, 1 \mathrm{H}), 7.20(\mathrm{~s}, 1 \mathrm{H}), 7.06\left(\mathrm{~d}, J_{\mathrm{H}-\mathrm{F}}=11.6 \mathrm{~Hz}, 1 \mathrm{H}\right)$, $6.65(\mathrm{~d}, J=15.2 \mathrm{~Hz}, 1 \mathrm{H}), 3.92(\mathrm{~s}, 3 \mathrm{H}), 3.51(\mathrm{~d}, J=12.6 \mathrm{~Hz}, 2 \mathrm{H}), 3.25(\mathrm{t}, J=11.7 \mathrm{~Hz}, 2 \mathrm{H}), 2.76(\mathrm{~m}, 4 \mathrm{H}), 2.54(\mathrm{~m}, 1 \mathrm{H})$, $2.03(\mathrm{~m}, 2 \mathrm{H}), 1.94(\mathrm{~m}, 2 \mathrm{H}), 1.85(\mathrm{~m}, 8 \mathrm{H}) \mathrm{ppm} ;{ }^{19} \mathrm{~F}$ NMR (376.5 MHz, $\left.\mathrm{CD}_{3} \mathrm{OD}\right): \delta=-125.4(\mathrm{~m}, 1 \mathrm{~F}),-147.6(\mathrm{~s}, 1 \mathrm{~F}) \mathrm{ppm}$; ${ }^{13} \mathrm{C} \mathrm{NMR}\left(100.0 \mathrm{MHz}, \mathrm{CD}_{3} \mathrm{OD}\right): \delta=180.8,178.7,160.6,155.3\left(\mathrm{dd}, J_{\mathrm{C}-\mathrm{F}}=6.1 \mathrm{~Hz},{ }^{1} J_{\mathrm{C}-\mathrm{F}}=244.7 \mathrm{~Hz}\right), 147.6,146.0(\mathrm{dd}$, 
$\left.J_{\mathrm{C}-\mathrm{F}}=7.6 \mathrm{~Hz},{ }^{1} J_{\mathrm{C}-\mathrm{F}}=247.4 \mathrm{~Hz}\right), 143.8,143.7,140.2\left(\mathrm{dd}, J_{\mathrm{C}-\mathrm{F}}=2.2 \mathrm{~Hz}, J_{\mathrm{C}-\mathrm{F}}=10.7 \mathrm{~Hz}\right), 133.0\left(\mathrm{dd}, J_{\mathrm{C}-\mathrm{F}}=9.5 \mathrm{~Hz}, J_{\mathrm{C}-\mathrm{F}}=\right.$ $14.9 \mathrm{~Hz}), 131.8,131.1,130.4,129.2,123.9,118.0\left(\mathrm{~d}, J_{\mathrm{C}-\mathrm{F}}=11.4 \mathrm{~Hz}\right), 116.4,114.4,109.6\left(\mathrm{dd}, J_{\mathrm{C}-\mathrm{F}}=2.0 \mathrm{~Hz}, J_{\mathrm{C}-\mathrm{F}}=24.5\right.$ $\mathrm{Hz}), 107.1,52.5,52.1\left(\mathrm{t}, J_{\mathrm{C}-\mathrm{F}}=3.6 \mathrm{~Hz}\right), 42.0,33.4,30.4,30.3,28.1,25.1,21.7 \mathrm{ppm}$; HRMS (ESI+): m/z calcd for $\mathrm{C}_{32} \mathrm{H}_{33} \mathrm{~F}_{2} \mathrm{~N}_{2} \mathrm{O}_{3}{ }^{+}[\mathrm{M}]^{+\circ}$ 531.2454, found 531.2461; HPLC (system A): $t_{\mathrm{R}}=4.8 \mathrm{~min}$ (purity $>99 \%$ at $600 \mathrm{~nm}$ ); LRMS (ESI + , recorded during RP-HPLC analysis): $m / z 530.8[\mathrm{M}]^{+{ }^{\circ}}(100)$, calcd for $\mathrm{C}_{32} \mathrm{H}_{33} \mathrm{~F}_{2} \mathrm{~N}_{2} \mathrm{O}_{3}{ }^{+} 531.3$; UV-vis (recorded during the HPLC analysis): $\lambda_{\max }=608 \mathrm{~nm}$ (broad peak).

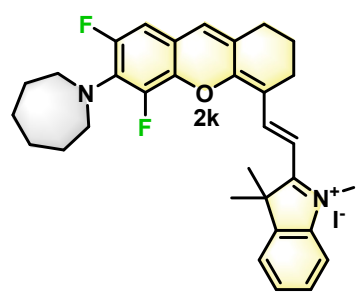

2k: 29\% yield; Dark green solid; ${ }^{1} \mathrm{H}$ NMR $\left(400.0 \mathrm{MHz}, \mathrm{CD}_{3} \mathrm{OD}\right): \delta=8.79(\mathrm{~d}, J=15.1 \mathrm{~Hz}, 1 \mathrm{H}), 7.68(\mathrm{~d}, J=7.2 \mathrm{~Hz}, 1 \mathrm{H})$, $7.60(\mathrm{~d}, J=7.5 \mathrm{~Hz}, 1 \mathrm{H}), 7.55(\mathrm{ddd}, J=1.2,7.3,7.9 \mathrm{~Hz}, 1 \mathrm{H}), 7.48(\mathrm{dt}, J=1.2,7.2 \mathrm{~Hz}, 1 \mathrm{H}), 7.23(\mathrm{~s}, 1 \mathrm{H}), 7.05\left(\mathrm{dd}, J_{\mathrm{H}-\mathrm{F}}=\right.$ $\left.1.7 \mathrm{~Hz}, J_{\mathrm{H}-\mathrm{F}}=12.3 \mathrm{~Hz}, 1 \mathrm{H}\right), 6.62(\mathrm{~d}, J=15.1 \mathrm{~Hz}, 1 \mathrm{H}), 3.91(\mathrm{~s}, 3 \mathrm{H}), 3.51(\mathrm{t}, J=5.6 \mathrm{~Hz}, 4 \mathrm{H}), 2.76(\mathrm{~m}, 4 \mathrm{H}), 1.94(\mathrm{~m}, 2 \mathrm{H})$, $1.83(\mathrm{~m}, 10 \mathrm{H}), 1.74(\mathrm{~m}, 4 \mathrm{H}) \mathrm{ppm} ;{ }^{19} \mathrm{~F} \mathrm{NMR}\left(376.5 \mathrm{MHz}, \mathrm{CD}_{3} \mathrm{OD}\right): \delta=-125.3\left(\mathrm{t}, J_{\mathrm{H}-\mathrm{F}}=9.0 \mathrm{~Hz}, 1 \mathrm{~F}\right),-146.5(\mathrm{~s}, 1 \mathrm{~F}) \mathrm{ppm}$; ${ }^{13} \mathrm{C}$ NMR (100.0 MHz, $\left.\mathrm{CD}_{3} \mathrm{OD}\right): \delta=180.4,161.0,154.9\left(\mathrm{~d},{ }^{1} J_{\mathrm{C}-\mathrm{F}}=242.7 \mathrm{~Hz}\right), 147.4,145.3\left(\mathrm{~d},{ }^{1} J_{\mathrm{C}-\mathrm{F}}=246.5 \mathrm{~Hz}\right), 143.8$, $143.7,140.6(\mathrm{~m}), 134.4\left(\mathrm{dd}, J_{\mathrm{C}-\mathrm{F}}=9.9 \mathrm{~Hz}, J_{\mathrm{C}-\mathrm{F}}=15.0 \mathrm{~Hz}\right), 132.4,130.4,130.2,128.9,123.9,116.6\left(\mathrm{~d}, J_{\mathrm{C}-\mathrm{F}}=11.1 \mathrm{~Hz}\right)$, $116.3,114.2,109.7\left(\mathrm{~d}, J_{\mathrm{C}-\mathrm{F}}=25.3 \mathrm{~Hz}\right), 106.6,55.1\left(\mathrm{t}, J_{\mathrm{C}-\mathrm{F}}=4.1 \mathrm{~Hz}\right), 52.3,33.4,31.0,30.3,28.8,28.2,25.1,21.7 \mathrm{ppm}$; HRMS (ESI+): $\mathrm{m} / \mathrm{z}$ calcd for $\mathrm{C}_{32} \mathrm{H}_{35} \mathrm{~F}_{2} \mathrm{~N}_{2} \mathrm{O}^{+}[\mathrm{M}]^{+\circ}$ 501.2712, found 501.2713; HPLC (system A): $t_{\mathrm{R}}=5.7$ min (purity $98 \%$ at $600 \mathrm{~nm}$ ); LRMS (ESI+, recorded during RP-HPLC analysis): $m / z 501.1[\mathrm{M}]^{+^{\circ}}$ (100), calcd for $\mathrm{C}_{32} \mathrm{H}_{35} \mathrm{~F}_{2} \mathrm{~N}_{2} \mathrm{O}^{+}$ 501.3; UV-vis (recorded during the HPLC analysis): $\lambda_{\max }=598 \mathrm{~nm}$ (broad peak).

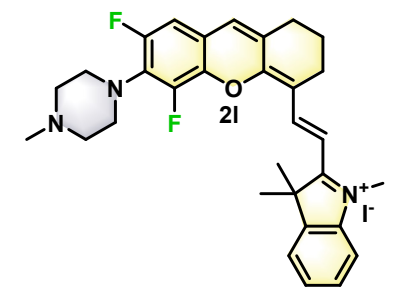

2I: $76 \%$ yield; Dark green solid; ${ }^{1} \mathrm{H}$ NMR $\left(400.0 \mathrm{MHz}, \mathrm{CD}_{3} \mathrm{OD}\right): \delta=8.77(\mathrm{~d}, J=15.4 \mathrm{~Hz}, 1 \mathrm{H}), 7.70(\mathrm{~d}, J=7.2 \mathrm{~Hz}, 1 \mathrm{H})$, $7.64(\mathrm{~d}, J=7.8 \mathrm{~Hz}, 1 \mathrm{H}), 7.57(\mathrm{dd}, J=7.2,7.6 \mathrm{~Hz}, 1 \mathrm{H}), 7.51(\mathrm{dd}, J=7.3,7.2 \mathrm{~Hz}, 1 \mathrm{H}), 7.17(\mathrm{~s}, 1 \mathrm{H}), 7.12\left(\mathrm{~d}, J_{\mathrm{H}-\mathrm{F}}=10.6\right.$ $\mathrm{Hz}, 1 \mathrm{H}), 6.71(\mathrm{~d}, J=15.3 \mathrm{~Hz}, 1 \mathrm{H}), 3.95$ (s, 3H), 3.63 (brs, 4H), 3.42 (brs, 4H), 2.96 (s, 3H), 2.76 (m, 4H), 1.94 (m, 2H), 1.82 (s, 6H) ppm; ${ }^{19} \mathrm{~F}$ NMR (376.5 MHz, CD 3 OD): $\delta=-125.9$ (m, 1F), -146.5 (s, 1F) ppm; ${ }^{13} \mathrm{C}$ NMR (100.0 MHz, $\left.\mathrm{CD}_{3} \mathrm{OD}\right): \delta=179.7,158.2,153.8\left(\mathrm{dd}, J_{\mathrm{C}-\mathrm{F}}=5.2 \mathrm{~Hz},{ }^{1} J_{\mathrm{C}-\mathrm{F}}=245.3 \mathrm{~Hz}\right), 146.2,144.9(\mathrm{~m}), 142.4,142.2,138.4\left(\mathrm{dd}, J_{\mathrm{C}-\mathrm{F}}=\right.$ $\left.2.3 \mathrm{~Hz}, J_{\mathrm{C}-\mathrm{F}}=10.7 \mathrm{~Hz}\right), 130.7,129.1,128.9,128.6\left(\mathrm{dd}, J_{\mathrm{C}-\mathrm{F}}=10.5 \mathrm{~Hz}, J_{\mathrm{C}-\mathrm{F}}=15.5 \mathrm{~Hz}\right), 127.9,122.4,118.2\left(\mathrm{~d}, J_{\mathrm{C}-\mathrm{F}}=10.9\right.$ $\mathrm{Hz}), 115.1,113.2,108.3\left(\mathrm{dd}, J_{\mathrm{C}-\mathrm{F}}=3.0 \mathrm{~Hz}, J_{\mathrm{C}-\mathrm{F}}=24.3 \mathrm{~Hz}\right.$ ), 106.5, 54.0, 51.1, 48.1, 42.8, 32.2, 28.9, 26.5, 23.5, $20.0 \mathrm{ppm}$; MS (ESI+): $\mathrm{m} / \mathrm{z}$ calcd for $\mathrm{C}_{31} \mathrm{H}_{34} \mathrm{~F}_{2} \mathrm{~N}_{3} \mathrm{O}^{+}[\mathrm{M}]^{+0} 502.2670$, found 502.2658; HPLC (system A): $t_{\mathrm{R}}=3.6$ min (purity $>99 \%$ at $600 \mathrm{~nm}$ ); LRMS (ESI+, recorded during RP-HPLC analysis): $m / z 272.3\left[2 \mathrm{M}+\mathrm{H}+\mathrm{CH}_{3} \mathrm{CN}\right]^{2+}(100)$ and $502.1[\mathrm{M}]^{+^{\circ}}$ (45), calcd for $\mathrm{C}_{31} \mathrm{H}_{34} \mathrm{~F}_{2} \mathrm{~N}_{3} \mathrm{O}^{+} 502.3$; UV-vis (recorded during the HPLC analysis): $\lambda_{\max }=591 \mathrm{~nm}$ (broad peak). 


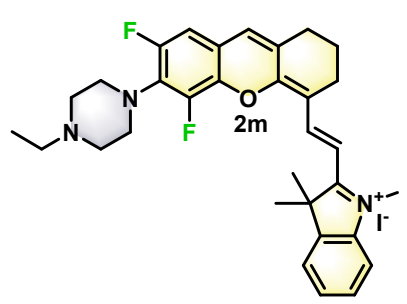

2m: $81 \%$ yield; Dark green solid; ${ }^{1} \mathrm{H}$ NMR $\left(400.0 \mathrm{MHz}, \mathrm{CD}_{3} \mathrm{OD}\right): \delta=8.78(\mathrm{~d}, J=15.3 \mathrm{~Hz}, 1 \mathrm{H}), 7.69(\mathrm{~d}, J=7.4 \mathrm{~Hz}$, $1 \mathrm{H}), 7.63(\mathrm{~d}, J=7.8 \mathrm{~Hz}, 1 \mathrm{H}), 7.56(\mathrm{ddd}, J=0.9,7.4,8.0 \mathrm{~Hz}, 1 \mathrm{H}), 7.50(\mathrm{dt}, J=0.7,7.4 \mathrm{~Hz}, 1 \mathrm{H}), 7.17(\mathrm{~s}, 1 \mathrm{H}), 7.10(\mathrm{dd}$, $\left.J_{\mathrm{H}-\mathrm{F}}=1,8 \mathrm{~Hz}, J_{\mathrm{H}-\mathrm{F}}=11.6 \mathrm{~Hz}, 1 \mathrm{H}\right), 6.68(\mathrm{~d}, J=15.3 \mathrm{~Hz}, 1 \mathrm{H}), 3.94(\mathrm{~s}, 3 \mathrm{H}), 3.55(\mathrm{t}, J=4.4 \mathrm{~Hz}, 4 \mathrm{H}), 3.12(\mathrm{brs}, 4 \mathrm{H}), 2.98$ $(\mathrm{m}, 2 \mathrm{H}), 2.76(\mathrm{~m}, 4 \mathrm{H}), 1.94(\mathrm{~m}, 2 \mathrm{H}), 1.82(\mathrm{~s}, 6 \mathrm{H}), 1.31(\mathrm{t}, J=7.3 \mathrm{~Hz}, 3 \mathrm{H}) \mathrm{ppm} ;{ }^{19} \mathrm{~F}$ NMR $\left(376.5 \mathrm{MHz}, \mathrm{CD}_{3} \mathrm{OD}\right): \delta=-$ $125.7(\mathrm{~m}, 1 \mathrm{~F}),-146.9$ (s, $1 \mathrm{~F}) \mathrm{ppm} ;{ }^{13} \mathrm{C} \mathrm{NMR}\left(100.0 \mathrm{MHz}, \mathrm{DMSO}-d_{6}\right): \delta=179.2,157.8,153.3\left(\mathrm{dd}, J_{\mathrm{C}-\mathrm{F}}=4.6 \mathrm{~Hz},{ }^{1} J_{\mathrm{C}-\mathrm{F}}=\right.$ $243.7 \mathrm{~Hz}), 144.4\left(\mathrm{dd}, J_{\mathrm{C}-\mathrm{F}}=7,6 \mathrm{~Hz},{ }^{1} J_{\mathrm{C}-\mathrm{F}}=247.7 \mathrm{~Hz}\right), 145.2,142.8,142.6,138.6(\mathrm{~m}), 130.1,129.9(\mathrm{~m}), 129.6,129.4$, $128.3,123.3,117.1\left(\mathrm{~d}, J_{\mathrm{C}-\mathrm{F}}=12.1 \mathrm{~Hz}\right), 115.0,114.3,109.1\left(\mathrm{~d}, J_{\mathrm{C}-\mathrm{F}}=24.6 \mathrm{~Hz}\right), 107.7,52.6,51.9,51.2,49.9,33.7,29.1$, 27.4, 23.9, 20.2, $11.2 \mathrm{ppm}$; MS (ESI + ): $\mathrm{m} / \mathrm{z}$ calcd for $\mathrm{C}_{32} \mathrm{H}_{36} \mathrm{~F}_{2} \mathrm{~N}_{3} \mathrm{O}^{+}[\mathrm{M}]^{+{ }^{\circ}}$ 516.2826, found 516.2890; HPLC (system A): $t_{\mathrm{R}}=3.7 \mathrm{~min}$ (purity $>99 \%$ at $600 \mathrm{~nm}$ ); LRMS (ESI+, recorded during RP-HPLC analysis): $\mathrm{m} / z 279.3[2 \mathrm{M}+\mathrm{H}+$ $\left.\mathrm{CH}_{3} \mathrm{CN}\right]^{2+}(100)$ and $516.1[\mathrm{M}]^{+{ }^{\circ}}(30)$, calcd for $\mathrm{C}_{32} \mathrm{H}_{36} \mathrm{~F}_{2} \mathrm{~N}_{3} \mathrm{O}^{+}$516.3; UV-vis (recorded during the HPLC analysis): $\lambda_{\max }$ $=592 \mathrm{~nm}$ (broad peak).

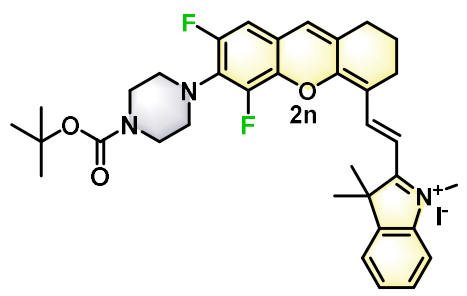

2n: 39\% yield; Dark green solid; ${ }^{1} \mathrm{H}$ NMR $\left(400.0 \mathrm{MHz}\right.$, DMSO- $\left.d_{6}\right): \delta=8.56(\mathrm{~d}, J=15.2 \mathrm{~Hz}, 1 \mathrm{H}), 7.80(\mathrm{~d}, J=7.2 \mathrm{~Hz}$, $1 \mathrm{H}), 7.74(\mathrm{~d}, J=7.9 \mathrm{~Hz}, 1 \mathrm{H}), 7.57$ (dd, $J=7.5,7.6 \mathrm{~Hz}, 1 \mathrm{H}), 7.50$ (dd, $J=7.3,7.4 \mathrm{~Hz}, 1 \mathrm{H}), 7.26(\mathrm{~m}, 2 \mathrm{H}), 6.71(\mathrm{~d}, J=$ $15.4 \mathrm{~Hz}, 1 \mathrm{H}), 3.94$ (s, 3H), 3.47 (brs, 4H), 3.21 (brs, 4H), 2.70 (m, 4H), $1.82(\mathrm{~m}, 2 \mathrm{H}), 1.75(\mathrm{~s}, 6 \mathrm{H}), 1.43(\mathrm{~s}, 9 \mathrm{H}) \mathrm{ppm} ;{ }^{19} \mathrm{~F}$ NMR (376.5 MHz, DMSO- $\left.d_{6}\right): \delta=-124.1(\mathrm{~m}, 1 \mathrm{~F}),-146.0(\mathrm{~s}, 1 \mathrm{~F}) \mathrm{ppm} ;{ }^{13} \mathrm{C}$ NMR $\left(100.0 \mathrm{MHz}, \mathrm{DMSO}-d_{6}\right): \delta=178.7$, $157.3,153.7,152.7\left(\mathrm{~d},{ }^{1} J_{\mathrm{C}-\mathrm{F}}=244.3 \mathrm{~Hz}\right), 144.8,143.9\left(\mathrm{~d},{ }^{1} J_{\mathrm{C}-\mathrm{F}}=247.8 \mathrm{~Hz}\right), 142.3,142.0,138.0\left(\mathrm{~d}, J_{\mathrm{C}-\mathrm{F}}=10.0 \mathrm{~Hz}\right), 129.9$ - $129.8(\mathrm{~m}), 129.6,129.0,128.8,127.7,122.6,116.6\left(\mathrm{~d}, J_{\mathrm{C}-\mathrm{F}}=11.2 \mathrm{~Hz}\right), 114.5,113.7,108.5\left(\mathrm{~d}, J_{\mathrm{C}-\mathrm{F}}=23.7 \mathrm{~Hz}\right), 107.1$, 79.0, 50.6, 50.4, 33.1, 28.9, 28.5, 28.0, 26.9, 23.4, $19.7 \mathrm{ppm}$; HRMS (ESI + ): $\mathrm{m} / \mathrm{z}$ calcd for $\mathrm{C}_{35} \mathrm{H}_{40} \mathrm{~F}_{2} \mathrm{~N}_{3} \mathrm{O}_{3}{ }^{+}[\mathrm{M}]^{+{ }^{\circ}} 588.3032$, found 588.3021; HPLC (system A): $t_{\mathrm{R}}=5.4 \mathrm{~min}$ (purity 97\% at $600 \mathrm{~nm}$ ); LRMS (ESI+, recorded during RP-HPLC analysis): $m / z 588.3[\mathrm{M}]^{+}(100)$, calcd for $\mathrm{C}_{35} \mathrm{H}_{40} \mathrm{~F}_{2} \mathrm{~N}_{3} \mathrm{O}_{3}{ }^{+} 588.3$; UV-vis (recorded during the HPLC analysis): $\lambda_{\max }=$ $602 \mathrm{~nm}$ (broad peak).

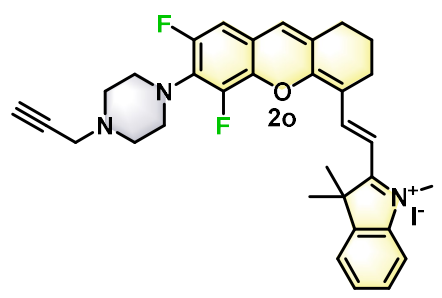

2o: $35 \%$ yield; Dark green solid; ${ }^{1} \mathrm{H}$ NMR $\left(600.0 \mathrm{MHz}\right.$, DMSO- $\left.d_{6}\right): \delta=8.56(\mathrm{~d}, J=14.9 \mathrm{~Hz}, 1 \mathrm{H}), 7.82(\mathrm{~d}, J=7.4 \mathrm{~Hz}$, $1 \mathrm{H}), 7.74(\mathrm{~d}, J=8.0 \mathrm{~Hz}, 1 \mathrm{H}), 7.57(\mathrm{dd}, J=7.6,7.8 \mathrm{~Hz}, 1 \mathrm{H}), 7.50(\mathrm{t}, J=7.4 \mathrm{~Hz}, 1 \mathrm{H}), 7.25(\mathrm{~m}, 2 \mathrm{H}), 6.70(\mathrm{~d}, J=15.3 \mathrm{~Hz}$, $1 \mathrm{H}), 3.94$ (s, 3H), 3.38 (brs, 2H), 3.30 (brs, 4H), 3.21 (brs, 1H), 2.71 (brs, 2H), 2.68 (brs, 2H), 2.61 (brs, 4H), 1.82 (m, 2H), 1.75 (s, 6H) ppm; ${ }^{19} \mathrm{~F}$ NMR (376.5 MHz, DMSO- $\left.d_{6}\right): \delta=-124.2(\mathrm{~s}, 1 \mathrm{~F}),-146.3(\mathrm{~s}, 1 \mathrm{~F}) \mathrm{ppm} ;{ }^{13} \mathrm{C}$ NMR $(100.0 \mathrm{MHz}$, 
DMSO- $\left.d_{6}\right): \delta=178.7,157.5,152.7\left(\mathrm{~d},{ }^{1} J_{\mathrm{C}-\mathrm{F}}=243.7 \mathrm{~Hz}\right), 144.7,143.7\left(\mathrm{dd}, J_{\mathrm{C}-\mathrm{F}}=7.8 \mathrm{~Hz},{ }^{1} J_{\mathrm{C}-\mathrm{F}}=247.0 \mathrm{~Hz}\right), 142.2,142.0$, $138.0\left(\mathrm{~d}, J_{\mathrm{C}-\mathrm{F}}=9.2 \mathrm{~Hz}\right), 130.0\left(\mathrm{dd}, J_{\mathrm{C}-\mathrm{F}}=8.4 \mathrm{~Hz}, J_{\mathrm{C}-\mathrm{F}}=11.3 \mathrm{~Hz}\right), 129.3(\mathrm{br}), 128.8,127.6,122.6,116.2\left(\mathrm{~d}, J_{\mathrm{C}-\mathrm{F}}=11.0\right.$ $\mathrm{Hz}), 114.4,113.6,108.5$ (d, $\left.J_{\mathrm{C}-\mathrm{F}}=24.5 \mathrm{~Hz}\right), 106.9,78.9,75.9,51.5,50.6,50.3,46.1,33.0,28.5,26.9,23.4,19.7 \mathrm{ppm}$; HRMS (ESI+): $\mathrm{m} / \mathrm{z}$ calcd for $\mathrm{C}_{33} \mathrm{H}_{34} \mathrm{~F}_{2} \mathrm{~N}_{3} \mathrm{O}^{+}[\mathrm{M}]^{+}$526.2664, found 526.2644; HPLC (system A): $t_{\mathrm{R}}=3.9$ min (purity $99 \%$ at $600 \mathrm{~nm}$ ); LRMS (ESI+, recorded during RP-HPLC analysis): $m / z 284.3\left[2 \mathrm{M}+\mathrm{H}+\mathrm{CH}_{3} \mathrm{CN}\right]^{+\circ}(100)$ and 526.0 $[\mathrm{M}]^{+\circ}(55)$, calcd for $\mathrm{C}_{33} \mathrm{H}_{34} \mathrm{~F}_{2} \mathrm{~N}_{3} \mathrm{O}^{+} 526.3$; UV-vis (recorded during the HPLC analysis): $\lambda_{\max }=591 \mathrm{~nm}$ (broad peak).

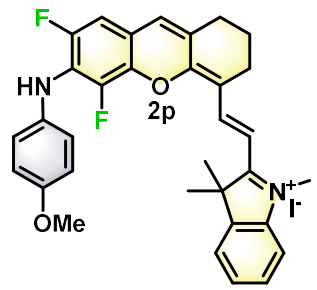

2p: 43\% yield; Dark green solid; ${ }^{1} \mathrm{H}$ NMR $\left(600.0 \mathrm{MHz}, \mathrm{CDCl}_{3}\right): \delta=8.67(\mathrm{~d}, J=15.3 \mathrm{~Hz}, 1 \mathrm{H}), 7.52(\mathrm{~m}, 1 \mathrm{H}), 7.44(\mathrm{~m}$, $3 \mathrm{H}), 7.00(\mathrm{~m}, 3 \mathrm{H}), 6.92\left(\mathrm{~d}, J_{\mathrm{H}-\mathrm{F}}=10.0 \mathrm{~Hz}, 1 \mathrm{H}\right), 6.89(\mathrm{~m}, 2 \mathrm{H}), 6.84(\mathrm{~d}, J=14.9 \mathrm{~Hz}, 1 \mathrm{H}), 5.89$ (brs, 1H), 4.15 (s, 3H), 3.83 (s, 3H), $2.91(\mathrm{t}, J=6.1 \mathrm{~Hz}, 2 \mathrm{H}), 2.74(\mathrm{t}, J=5.7 \mathrm{~Hz}, 2 \mathrm{H}), 1.96(\mathrm{~m}, 2 \mathrm{H}), 1.74(\mathrm{~s}, 6 \mathrm{H}) \mathrm{ppm} ;{ }^{19} \mathrm{~F}$ NMR $\left(376.5 \mathrm{MHz}, \mathrm{CD}_{3} \mathrm{OD}\right)$ : $\delta=-128.0(\mathrm{~m}, 1 \mathrm{~F}),-147.3(\mathrm{~s}, 1 \mathrm{~F}) \mathrm{ppm} ;{ }^{13} \mathrm{C} \mathrm{NMR}\left(100.0 \mathrm{MHz}, \mathrm{CD}_{3} \mathrm{OD}\right): \delta=180.1,161.1,157.2,152.4\left(\mathrm{dd}, J_{\mathrm{C}-\mathrm{F}}=6.5\right.$ $\left.\mathrm{Hz},{ }^{1} J_{\mathrm{C}-\mathrm{F}}=244.1 \mathrm{~Hz}\right), 147.1,143.6,143.5,140.5\left(\mathrm{dd}, J_{\mathrm{C}-\mathrm{F}}=2.0 \mathrm{~Hz}, J_{\mathrm{C}-\mathrm{F}}=9.2 \mathrm{~Hz}\right), 136.4,132.9,130.2,129.6,128.7$, $126.9\left(\mathrm{dd}, J_{\mathrm{C}-\mathrm{F}}=10.1 \mathrm{~Hz}, J_{\mathrm{C}-\mathrm{F}}=16.5 \mathrm{~Hz}\right), 123.7,121.9,116.1,115.4\left(\mathrm{~d}, J_{\mathrm{C}-\mathrm{F}}=10.3 \mathrm{~Hz}\right), 115.1,113.9,109.2\left(\mathrm{dd}, J_{\mathrm{C}-\mathrm{F}}=\right.$ $\left.2.5 \mathrm{~Hz}, J_{\mathrm{C}-\mathrm{F}}=22.7 \mathrm{~Hz}\right), 106.0,56.0,52.1,32.9,30.1,27.9,25.0,21.6 \mathrm{ppm}$; HRMS (ESI+): $\mathrm{m} / \mathrm{z}$ calcd for $\mathrm{C}_{33} \mathrm{H}_{31} \mathrm{~F}_{2} \mathrm{~N}_{2} \mathrm{O}_{2}{ }^{+}$ $[\mathrm{M}]^{+^{\circ}} 525.2348$, found 525.2348; HPLC (system A): $t_{\mathrm{R}}=5.1 \mathrm{~min}$ (purity $99 \%$ at $600 \mathrm{~nm}$ ); LRMS (ESI+, recorded during RP-HPLC analysis): $m / z 525.0[\mathrm{M}]^{+{ }^{\circ}}$ (100), calcd for $\mathrm{C}_{33} \mathrm{H}_{31} \mathrm{~F}_{2} \mathrm{~N}_{2} \mathrm{O}_{2}{ }^{+} 525.2$; UV-vis (recorded during the HPLC analysis): $\lambda_{\max }=626 \mathrm{~nm}$ (broad peak).

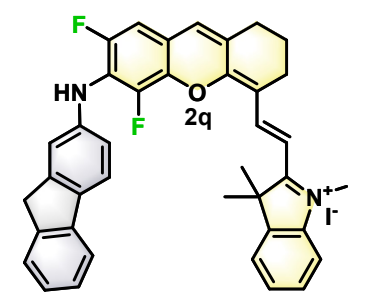

2q: 45\% yield; Dark green solid; ${ }^{1} \mathrm{H}$ NMR $\left(400.0 \mathrm{MHz}, \mathrm{CD}_{3} \mathrm{OD}\right): \delta=8.74(\mathrm{~d}, J=15.2 \mathrm{~Hz}, 1 \mathrm{H}), 7.68$ (dd, $J=8.1,8.5$ $\mathrm{Hz}, 2 \mathrm{H}), 7.56(\mathrm{~d}, J=7.9 \mathrm{~Hz}, 2 \mathrm{H}), 7.50(\mathrm{~m}, 2 \mathrm{H}), 7.40(\mathrm{~m}, 1 \mathrm{H}), 7.32(\mathrm{t}, J=7.4 \mathrm{~Hz}, 1 \mathrm{H}), 7.19(\mathrm{~m}, 4 \mathrm{H}), 6.98\left(\mathrm{~d}, J_{\mathrm{H}-\mathrm{F}}=8.0\right.$ $\mathrm{Hz}, 1 \mathrm{H}), 6.60(\mathrm{~d}, J=15.1 \mathrm{~Hz}, 1 \mathrm{H}), 3.88(\mathrm{~s}, 3 \mathrm{H}), 3.82(\mathrm{~s}, 2 \mathrm{H}), 2.77(\mathrm{t}, J=5.8 \mathrm{~Hz}, 2 \mathrm{H}), 2.71(\mathrm{t}, J=5.9 \mathrm{~Hz}, 2 \mathrm{H}), 1.94(\mathrm{~m}$, $2 \mathrm{H}), 1.73(\mathrm{~s}, 6 \mathrm{H}) \mathrm{ppm} ;{ }^{19} \mathrm{~F}$ NMR $\left(376.5 \mathrm{MHz}, \mathrm{CD}_{3} \mathrm{OD}\right): \delta=-126.7$ (m, $\left.1 \mathrm{~F}\right),-144.9(\mathrm{~s}, 1 \mathrm{~F}) \mathrm{ppm} ;{ }^{13} \mathrm{C} \mathrm{NMR}(100.0 \mathrm{MHz}$, $\left.\mathrm{CD}_{3} \mathrm{OD}\right): \delta=180.5,160.8,153.4\left(\mathrm{dd}, J_{\mathrm{C}-\mathrm{F}}=5.7 \mathrm{~Hz},{ }^{1} J_{\mathrm{C}-\mathrm{F}}=244.5 \mathrm{~Hz}\right), 147.4,145.7,144.8\left(\mathrm{~d}, J_{\mathrm{C}-\mathrm{F}}=6.2 \mathrm{~Hz}\right), 144.2$, $143.7,143.1,143.0,140.4\left(\mathrm{~d}, J_{\mathrm{C}-\mathrm{F}}=9.9 \mathrm{~Hz}\right), 137.3,130.5,130.3,129.0,128.0,127.0,126.0,125.3\left(\mathrm{dd}, J_{\mathrm{C}-\mathrm{F}}=11.0 \mathrm{~Hz}\right.$, $\left.J_{\mathrm{C}-\mathrm{F}}=16.9 \mathrm{~Hz}\right), 123.9,121.1,120.0,118.1,117.1\left(\mathrm{~d}, J_{\mathrm{C}-\mathrm{F}}=10.3 \mathrm{~Hz}\right), 116.3,115.7,114.2,109.5\left(\mathrm{~d}, J_{\mathrm{C}-\mathrm{F}}=22.8 \mathrm{~Hz}\right), 106.7$, 52.3, 37.8, 33.2, 30.3, 28.0, 25.0, 21.7 ppm; HRMS (ESI+): $\mathrm{m} / \mathrm{z}$ calcd for $\mathrm{C}_{39} \mathrm{H}_{33} \mathrm{~F}_{2} \mathrm{~N}_{2} \mathrm{O}^{+}[\mathrm{M}]^{+\circ} 583.2555$ found 583.2541.

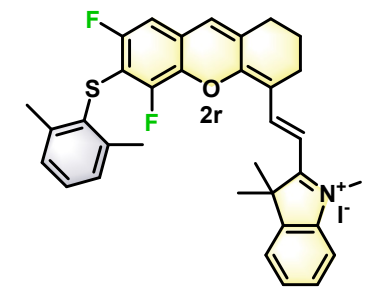


2r: $86 \%$ yield; Dark green solid; ${ }^{1} \mathrm{H}$ NMR (600.0 MHz, $\left.\mathrm{CD}_{3} \mathrm{OD}\right): \delta=8.72(\mathrm{~d}, J=15.5 \mathrm{~Hz}, 1 \mathrm{H}), 7.72(\mathrm{~d}, J=7.2 \mathrm{~Hz}, 1 \mathrm{H})$, $7.67(\mathrm{~d}, J=8.0 \mathrm{~Hz}, 1 \mathrm{H}), 7.59(\mathrm{dt}, J=1.0,7.6 \mathrm{~Hz}, 1 \mathrm{H}), 7.54(\mathrm{dt}, J=0.7,7.5 \mathrm{~Hz}, 1 \mathrm{H}), 7.20(\mathrm{~m}, 1 \mathrm{H}), 7.15(\mathrm{~s}, 1 \mathrm{H}), 7.13(\mathrm{~d}$, $J=11.7 \mathrm{~Hz}, 2 \mathrm{H}), 7.05\left(\mathrm{dd}, J_{\mathrm{H}-\mathrm{F}}=1.4,9.1 \mathrm{~Hz}, 1 \mathrm{H}\right), 6.76(\mathrm{~d}, J=15.4 \mathrm{~Hz}, 1 \mathrm{H}), 3.96(\mathrm{~s}, 3 \mathrm{H}), 2.77(\mathrm{t}, J=5.7 \mathrm{~Hz}, 2 \mathrm{H}), 2.73$ (t, $J=6.0 \mathrm{~Hz}, 2 \mathrm{H}), 2.47(\mathrm{~s}, 6 \mathrm{H}), 1.94(\mathrm{~m}, 2 \mathrm{H}), 1.79(\mathrm{~s}, 6 \mathrm{H}) \mathrm{ppm} ;{ }^{19} \mathrm{~F}$ NMR $\left(376.5 \mathrm{MHz}\right.$, DMSO-$\left.d_{6}\right): \delta=-112.7\left(\mathrm{~d}, J_{\mathrm{H}-\mathrm{F}}\right.$ $=9.1 \mathrm{~Hz}, 1 \mathrm{~F}),-131.3(\mathrm{~s}, 1 \mathrm{~F}) \mathrm{ppm} ;{ }^{13} \mathrm{C}$ NMR $\left(100.0 \mathrm{MHz}, \mathrm{DMSO}-d_{6}\right): \delta=179.4,156.8\left(\mathrm{dd}, J_{\mathrm{C}-\mathrm{F}}=3.6 \mathrm{~Hz},{ }^{1} J_{\mathrm{C}-\mathrm{F}}=244.0\right.$ $\mathrm{Hz}), 156.1,148.1\left(\mathrm{dd}, J_{\mathrm{C}-\mathrm{F}}=6.5 \mathrm{~Hz},{ }^{1} J_{\mathrm{C}-\mathrm{F}}=248.0 \mathrm{~Hz}\right), 145.0,142.6,142.1,142.0,137.0\left(\mathrm{dd}, J_{\mathrm{C}-\mathrm{F}}=2.5 \mathrm{~Hz}, J_{\mathrm{C}-\mathrm{F}}=11.9\right.$ $\mathrm{Hz}), 132.5,129.6,129.3,129.0,128.6,128.2,127.5,122.8,122.6\left(\mathrm{dd}, J_{\mathrm{C}-\mathrm{F}}=1.6 \mathrm{~Hz}, J_{\mathrm{C}-\mathrm{F}}=10.8 \mathrm{~Hz}\right), 115.0,114.2,113.7$ $\left(\mathrm{dd}, J_{\mathrm{C}-\mathrm{F}}=17.4 \mathrm{~Hz}, J_{\mathrm{C}-\mathrm{F}}=23.3 \mathrm{~Hz}\right), 108.6,108.5(\mathrm{~m}), 51.0,33.5,28.8,26.7,23.4,21.3,19.7 \mathrm{ppm} ; \mathrm{HRMS}(\mathrm{ESI}+): \mathrm{m} / \mathrm{z}$ calcd for $\mathrm{C}_{34} \mathrm{H}_{32} \mathrm{~F}_{2} \mathrm{NOS}^{+}[\mathrm{M}]^{+\circ} 540.2167$, found 540.2158 .

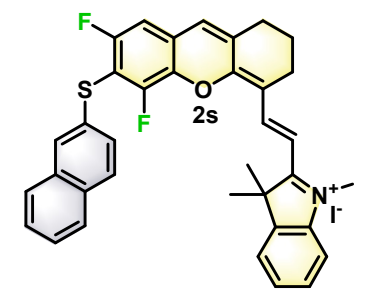

2s: $71 \%$ yield; Dark green solid; ${ }^{1} \mathrm{H}$ NMR $\left(400.0 \mathrm{MHz}\right.$, DMSO- $\left.d_{6}\right): \delta=8.51(\mathrm{~d}, J=15.5 \mathrm{~Hz}, 1 \mathrm{H}), 7.88(\mathrm{~d}, J=8.5 \mathrm{~Hz}$, 2H), $7.82(\mathrm{~m}, 2 \mathrm{H}), 7.77(\mathrm{dd}, J=8.1,8.5 \mathrm{~Hz}, 2 \mathrm{H}), 7.58(\mathrm{t}, J=7.3 \mathrm{~Hz}, 1 \mathrm{H}), 7.46(\mathrm{~m}, 3 \mathrm{H}), 7.42(\mathrm{~d}, J=8.5 \mathrm{~Hz}, 1 \mathrm{H}), 7.36$ $\left(\mathrm{dd}, J_{\mathrm{H}-\mathrm{F}}=1.8 \mathrm{~Hz}, J_{\mathrm{H}-\mathrm{F}}=8.7 \mathrm{~Hz}, 1 \mathrm{H}\right), 7.25(\mathrm{~s}, 1 \mathrm{H}), 6.82(\mathrm{~d}, J=15.6 \mathrm{~Hz}, 1 \mathrm{H}), 3.99(\mathrm{~s}, 3 \mathrm{H}), 2.70(\mathrm{~m}, 4 \mathrm{H}), 1.83(\mathrm{~m}, 2 \mathrm{H})$, 1.69 (s, 6H) ppm; ${ }^{19} \mathrm{~F}$ NMR (376.5 MHz, DMSO- $\left.d_{6}\right): \delta=-110.6$ (m, 1F), -128.4 (s, 1F) ppm; ${ }^{13} \mathrm{C}$ NMR $(100.0 \mathrm{MHz}$, DMSO- $\left.d_{6}\right): \delta=179.5,157.6\left(\mathrm{dd}, J_{\mathrm{C}-\mathrm{F}}=2.4 \mathrm{~Hz},{ }^{1} J_{\mathrm{C}-\mathrm{F}}=244.4 \mathrm{~Hz}\right), 155.7,149.4\left(\mathrm{dd}, J_{\mathrm{C}-\mathrm{F}}=4.7 \mathrm{~Hz},{ }^{1} J_{\mathrm{C}-\mathrm{F}}=248.5 \mathrm{~Hz}\right)$, $144.9,142.6,142.0,137.2\left(\mathrm{dd}, J_{\mathrm{C}-\mathrm{F}}=2.6 \mathrm{~Hz}, J_{\mathrm{C}-\mathrm{F}}=12.1 \mathrm{~Hz}\right), 133.4,133.2,131.6,131.3,129.2,128.9,128.3,127.7$, $127.1,127.0,126.4,126.2,125.7,124.8\left(\mathrm{dd}, J_{\mathrm{C}-\mathrm{F}}=2.2 \mathrm{~Hz}, J_{\mathrm{C}-\mathrm{F}}=11.1 \mathrm{~Hz}\right), 122.7,115.2,114.3,110.1\left(\mathrm{dd}, J_{\mathrm{C}-\mathrm{F}}=18.6 \mathrm{~Hz}\right.$, $\left.J_{\mathrm{C}-\mathrm{F}}=24.8 \mathrm{~Hz}\right), 108.9,108.6(\mathrm{~m}), 51.0,33.6,28.8,26.6,23.3,19.6 \mathrm{ppm} ; \mathrm{HRMS}(\mathrm{ESI}+): \mathrm{m} / \mathrm{z}$ calcd for $\mathrm{C}_{36} \mathrm{H}_{30} \mathrm{~F}_{2} \mathrm{NOS}^{+}$ $[\mathrm{M}]^{+\circ} 562.2011$, found 562.1994 .

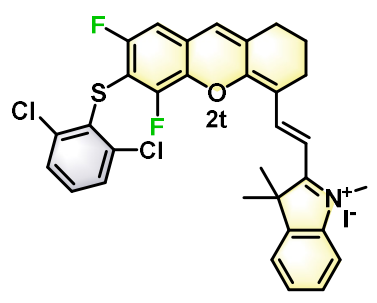

2t: $92 \%$ yield; Dark green solid; ${ }^{1} \mathrm{H}$ NMR (400.0 MHz, DMSO- $\left.d_{6}\right): \delta=8.50(\mathrm{~d}, J=15.6 \mathrm{~Hz}, 1 \mathrm{H}), 7.84(\mathrm{~d}, J=7.1 \mathrm{~Hz}$, $1 \mathrm{H}), 7.79(\mathrm{~d}, J=7.8 \mathrm{~Hz}, 1 \mathrm{H}), 7.60(\mathrm{~m}, 3 \mathrm{H}), 7.54(\mathrm{dd}, J=7.3,7.4 \mathrm{~Hz}, 1 \mathrm{H}), 7.47(\mathrm{dd}, J=7.7,8.5 \mathrm{~Hz}, 1 \mathrm{H}), 7.30\left(\mathrm{~d}, J_{\mathrm{H}-\mathrm{F}}=\right.$ $9.0 \mathrm{~Hz}, 1 \mathrm{H}), 7.19(\mathrm{~s}, 1 \mathrm{H}), 6.82(\mathrm{~d}, J=15.6 \mathrm{~Hz}, 1 \mathrm{H}), 3.98(\mathrm{~s}, 3 \mathrm{H}), 2.71(\mathrm{~m}, 4 \mathrm{H}), 1.82(\mathrm{~m}, 2 \mathrm{H}), 1.73(\mathrm{~s}, 6 \mathrm{H}) \mathrm{ppm} ;{ }^{19} \mathrm{~F} \mathrm{NMR}$ (376.5 MHz, DMSO- $\left.d_{6}\right): \delta=-112.4\left(\mathrm{~d}, J_{\mathrm{H}-\mathrm{F}}=9.3 \mathrm{~Hz}, 1 \mathrm{~F}\right),-130.2(\mathrm{~s}, 1 \mathrm{~F}) \mathrm{ppm} ;{ }^{13} \mathrm{C}$ NMR $\left(100.0 \mathrm{MHz}, \mathrm{DMSO}-d_{6}\right): \delta=$ $179.5,156.5\left(\mathrm{dd}, J_{\mathrm{C}-\mathrm{F}}=2.5 \mathrm{~Hz},{ }^{1} J_{\mathrm{C}-\mathrm{F}}=244.9 \mathrm{~Hz}\right), 155.9,148.2\left(\mathrm{dd}, J_{\mathrm{C}-\mathrm{F}}=5.3 \mathrm{~Hz},{ }^{1} J_{\mathrm{C}-\mathrm{F}}=249.2 \mathrm{~Hz}\right), 145.0,142.6,142.1$, $138.9,137.0\left(\mathrm{dd}, J_{\mathrm{C}-\mathrm{F}}=2.0 \mathrm{~Hz}, J_{\mathrm{C}-\mathrm{F}}=10.8 \mathrm{~Hz}\right), 133.0,131.9,129.4,128.9,128.8,128.3,127.1,123.4\left(\mathrm{dd}, J_{\mathrm{C}-\mathrm{F}}=2.2 \mathrm{~Hz}\right.$, $\left.J_{\mathrm{C}-\mathrm{F}}=11.0 \mathrm{~Hz}\right), 122.8,115.2,114.2,111.8\left(\mathrm{dd}, J_{\mathrm{C}-\mathrm{F}}=16.9 \mathrm{~Hz}, J_{\mathrm{C}-\mathrm{F}}=22.9 \mathrm{~Hz}\right), 108.8,108.6\left(\mathrm{dd}, J_{\mathrm{C}-\mathrm{F}}=2.7 \mathrm{~Hz}, J_{\mathrm{C}-\mathrm{F}}=\right.$ $24.7 \mathrm{~Hz}$ ), 51.1, 33.5, 28.8, 26.6, 23.4, $19.6 \mathrm{ppm}$; HRMS (ESI+): $\mathrm{m} / \mathrm{z}$ calcd for $\mathrm{C}_{32} \mathrm{H}_{26} \mathrm{Cl}_{2} \mathrm{~F}_{2} \mathrm{NOS}^{+}[\mathrm{M}]^{+^{\circ}}$ 580.1075, found 580.1072. 


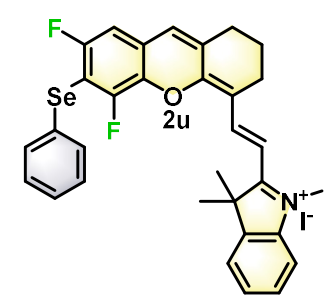

2u: 95\% yield; Dark green solid; ${ }^{1} \mathrm{H}$ NMR (400.0 MHz, DMSO- $\left.d_{6}\right): \delta=8.52(\mathrm{~d}, J=15.5 \mathrm{~Hz}, 1 \mathrm{H}), 7.81(\mathrm{dd}, J=6.1,6.8$ $\mathrm{Hz}, 2 \mathrm{H}), 7.58$ (dd, $J=7.3,7.8 \mathrm{~Hz}, 1 \mathrm{H}), 7.53$ (dd, $J=7.2,7.4 \mathrm{~Hz}, 1 \mathrm{H}), 7.40(\mathrm{~m}, 3 \mathrm{H}), 7.30(\mathrm{~m}, 3 \mathrm{H}), 7.25(\mathrm{~s}, 1 \mathrm{H}), 6.83$ (d, $J=15.6 \mathrm{~Hz}, 1 \mathrm{H}), 4.00(\mathrm{~s}, 3 \mathrm{H}), 2.70(\mathrm{~m}, 4 \mathrm{H}), 1.82(\mathrm{brs}, 2 \mathrm{H}), 1.72(\mathrm{~s}, 6 \mathrm{H}) \mathrm{ppm} ;{ }^{19} \mathrm{~F}$ NMR $\left(376.5 \mathrm{MHz}, \mathrm{DMSO}-d_{6}\right): \delta=-$ $105.1\left(\mathrm{~d}, J_{\mathrm{H}-\mathrm{F}}=7.8 \mathrm{~Hz}, 1 \mathrm{~F}\right),-122.6(\mathrm{~s}, 1 \mathrm{~F}) \mathrm{ppm} ;{ }^{13} \mathrm{C} \mathrm{NMR}\left(100.0 \mathrm{MHz}, \mathrm{DMSO}-d_{6}\right): \delta=179.5,157.4\left(\mathrm{dd}, J_{\mathrm{C}-\mathrm{F}}=4.3 \mathrm{~Hz}\right.$, $\left.{ }^{1} J_{\mathrm{C}-\mathrm{F}}=241.5 \mathrm{~Hz}\right), 155.9,149.2\left(\mathrm{dd}, J_{\mathrm{C}-\mathrm{F}}=6.6 \mathrm{~Hz},{ }^{1} J_{\mathrm{C}-\mathrm{F}}=245.5 \mathrm{~Hz}\right), 144.9,142.6,142.0,136.8\left(\mathrm{dd}, J_{\mathrm{C}-\mathrm{F}}=2.9 \mathrm{~Hz}, J_{\mathrm{C}-\mathrm{F}}=\right.$ $13.1 \mathrm{~Hz}), 133.1,130.7,129.7,129.6,128.9,128.3,127.5,127.3,124.8\left(\mathrm{dd}, J_{\mathrm{C}-\mathrm{F}}=2.2 \mathrm{~Hz}, J_{\mathrm{C}-\mathrm{F}}=10.9 \mathrm{~Hz}\right), 122.8,115.1$, $114.3,108.8,108.4\left(\mathrm{dd}, J_{\mathrm{C}-\mathrm{F}}=2.9 \mathrm{~Hz}, J_{\mathrm{C}-\mathrm{F}}=27.3 \mathrm{~Hz}\right), 106.8\left(\mathrm{dd}, J_{\mathrm{C}-\mathrm{F}}=23.01 \mathrm{~Hz}, J_{\mathrm{C}-\mathrm{F}}=29.5 \mathrm{~Hz}\right), 51.0,33.5,28.8,26.7$, 23.3, 19.6 ppm; HRMS (ESI+): m/z calcd for $\mathrm{C}_{32} \mathrm{H}_{28} \mathrm{~F}_{2} \mathrm{NOSe}^{+}[\mathrm{M}]^{+\circ}$ 560.1301, found 560.1290; HPLC (system A): $t_{\mathrm{R}}=$ $5.3 \mathrm{~min}$ (purity $97 \%$ at $600 \mathrm{~nm}$ ); LRMS (ESI+, recorded during RP-HPLC analysis): $\mathrm{m} / z 558.1$ (60) and 560.1 (100) $[\mathrm{M}]^{+\circ}$ (two major Se isotopes), calcd for $\mathrm{C}_{32} \mathrm{H}_{28} \mathrm{~F}_{2} \mathrm{NOSe}^{+} 560.1$; UV-vis (recorded during the HPLC analysis): $\lambda_{\max }=553$ $\mathrm{nm}$ (broad peak).

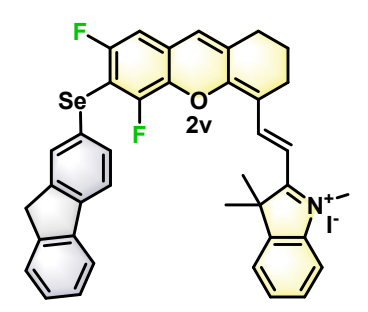

2v: 66\% yield; Dark green solid; ${ }^{1} \mathrm{H}$ NMR (400.0 MHz, $\left.\mathrm{CDCl}_{3}\right): \delta=8.35(\mathrm{~d}, J=15.5 \mathrm{~Hz}, 1 \mathrm{H}), 7.76(\mathrm{~d}, J=7.5 \mathrm{~Hz}, 1 \mathrm{H})$, $7.73(\mathrm{~s}, 1 \mathrm{H}), 7.70(\mathrm{~d}, J=8.0 \mathrm{~Hz}, 1 \mathrm{H}), 7.58(\mathrm{~d}, J=7.9 \mathrm{~Hz}, 1 \mathrm{H}), 7.52(\mathrm{~m}, 2 \mathrm{H}), 7.46(\mathrm{~m}, 2 \mathrm{H}), 7.39(\mathrm{~m}, 2 \mathrm{H}), 7.32(\mathrm{dt}, J=$ 1.0, $7.5 \mathrm{~Hz}, 1 \mathrm{H}), 7.04(\mathrm{~d}, J=15.4 \mathrm{~Hz}, 1 \mathrm{H}), 6.87(\mathrm{~m}, 2 \mathrm{H}), 4.25(\mathrm{~s}, 3 \mathrm{H}), 3.88(\mathrm{~s}, 2 \mathrm{H}), 2.95(\mathrm{t}, J=6.0 \mathrm{~Hz}, 2 \mathrm{H}), 2.72(\mathrm{t}, J=$ $5.7 \mathrm{~Hz}, 2 \mathrm{H}), 1.94(\mathrm{~m}, 2 \mathrm{H}), 1.76(\mathrm{~s}, 6 \mathrm{H}) \mathrm{ppm} ;{ }^{19} \mathrm{~F}$ NMR $\left(376.5 \mathrm{MHz}, \mathrm{DMSO}-d_{6}\right): \delta=-105.1(\mathrm{~s}, 1 \mathrm{~F}),-122.7$ (s, $\left.1 \mathrm{~F}\right) \mathrm{ppm}$; ${ }^{13} \mathrm{C}$ NMR $\left(100.0 \mathrm{MHz}\right.$, DMSO- $\left.d_{6}\right): \delta=179.3,157.3\left(\mathrm{dd}, J_{\mathrm{C}-\mathrm{F}}=3.8 \mathrm{~Hz},{ }^{1} J_{\mathrm{C}-\mathrm{F}}=241.8 \mathrm{~Hz}\right), 155.8,149.0\left(\mathrm{dd}, J_{\mathrm{C}-\mathrm{F}}=6.3\right.$ $\left.\mathrm{Hz},{ }^{1} J_{\mathrm{C}-\mathrm{F}}=245.7 \mathrm{~Hz}\right), 144.8,144.4,142.8,142.5,142.0,140.8,140.0,136.7\left(\mathrm{dd}, J_{\mathrm{C}-\mathrm{F}}=2.5 \mathrm{~Hz}, J_{\mathrm{C}-\mathrm{F}}=12.9 \mathrm{~Hz}\right), 132.8$, $130.0,128.9,128.2,128.1,127.3,127.2,126.8,125.0,124.4$ (d, $\left.J_{\mathrm{C}-\mathrm{F}}=10.8 \mathrm{~Hz}\right), 122.7,120.9,120.1,115.0,114.2,108.7$, $108.3\left(\mathrm{~d}, J_{\mathrm{C}-\mathrm{F}}=27.5 \mathrm{~Hz}\right), 107.3\left(\mathrm{dd}, J_{\mathrm{C}-\mathrm{F}}=22.9 \mathrm{~Hz}, J_{\mathrm{C}-\mathrm{F}}=29.5 \mathrm{~Hz}\right), 50.9,36.3,33.6,28.7,26.7,23.3,19.5 \mathrm{ppm}$; HRMS (ESI+): $\mathrm{m} / \mathrm{z}$ calcd for $\mathrm{C}_{39} \mathrm{H}_{32} \mathrm{~F}_{2} \mathrm{NOSe}^{+}[\mathrm{M}]^{+\circ}$ 648.1615, found 648.1643 .

\section{References}

1. X. Zou, L. Yang, X. Liu, H. Sun, H. Lu, Adv. Synth. Catal. 2015, 357, 3040 - 3046.

2. M. J. H. Ong, R. Srinivasan, A. Romieu, J. A. Richard, Org. Lett. 2016, 18, 5122-5125.

3. N. B. Yapici, S. Mandalapu, L. Bi, Bioorg. Med. Chem. Lett. 2015, 25, 3476-3480. 


\section{5. ${ }^{1} \mathrm{H},{ }^{19} \mathrm{~F}$ and ${ }^{13} \mathrm{C}$ NMR spectra of synthesized compounds}
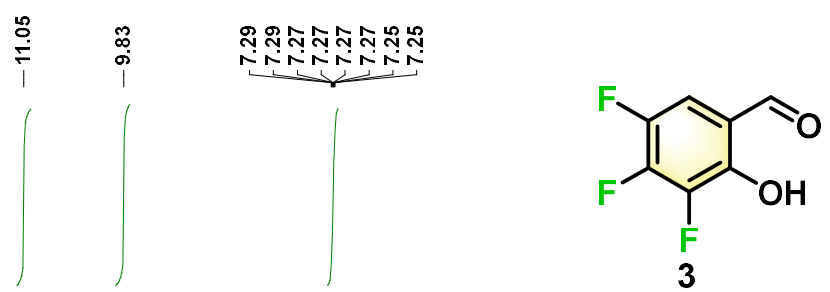

${ }^{1} \mathrm{H} \mathrm{NMR}\left(400.0 \mathrm{MHz}, \mathrm{CDCl}_{3}\right)$
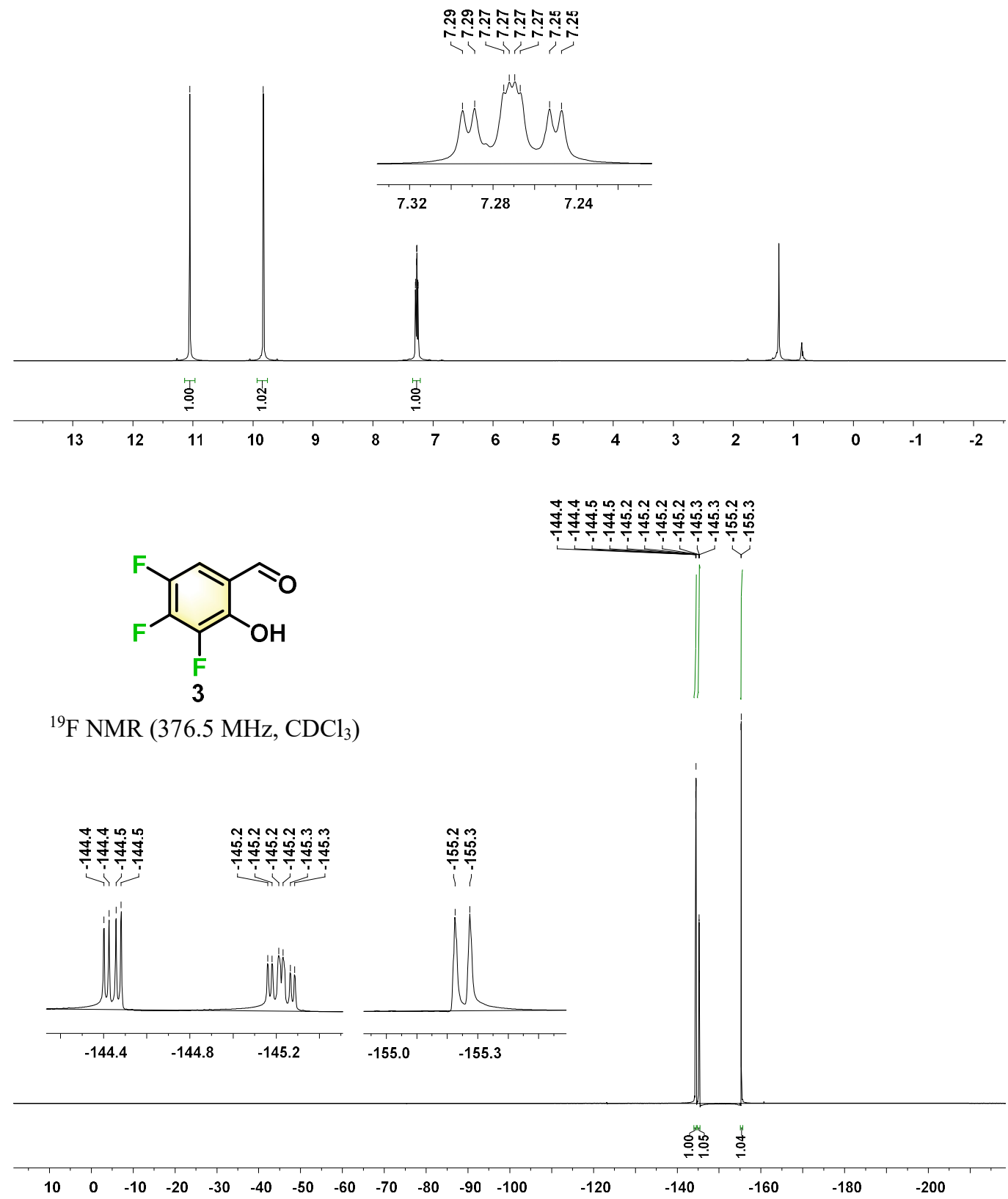

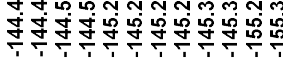




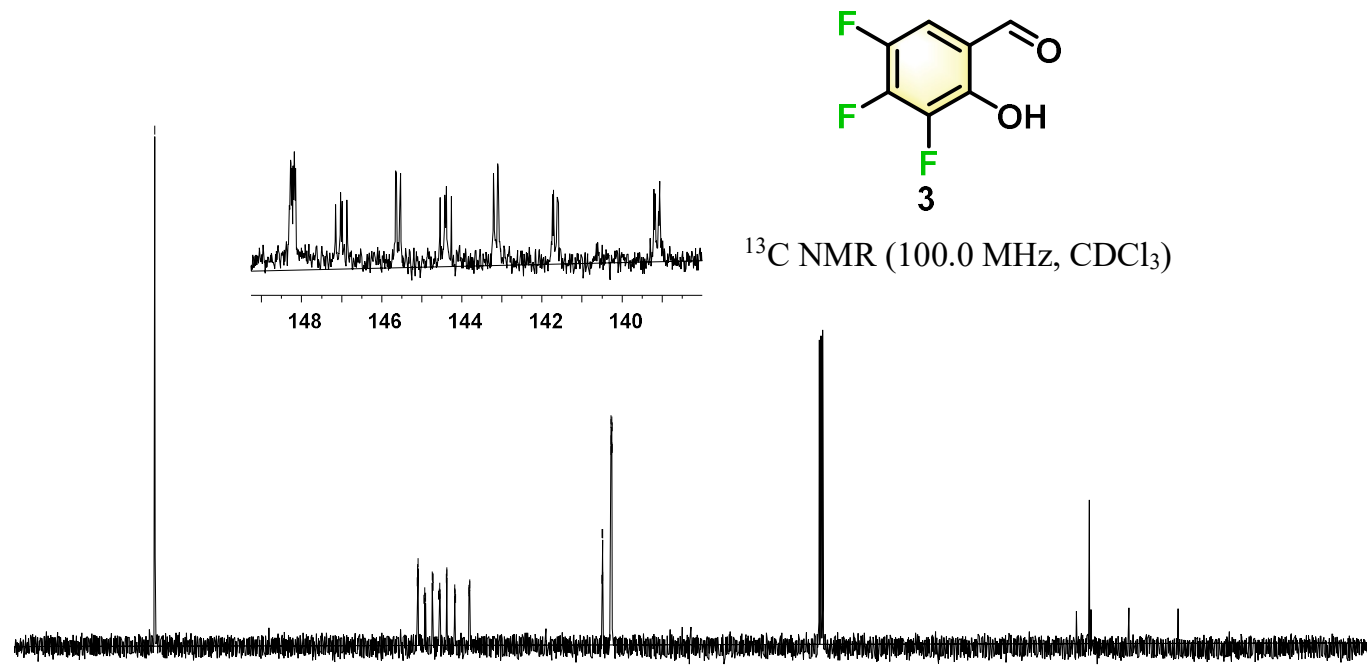

$\begin{array}{lllllllllllllllllllllll}210 & 200 & 190 & 180 & 170 & 160 & 150 & 140 & 130 & 120 & 110 & 100 & 90 & 80 & 70 & 60 & 50 & 40 & 30 & 20 & 10 & 0 & -10\end{array}$ 

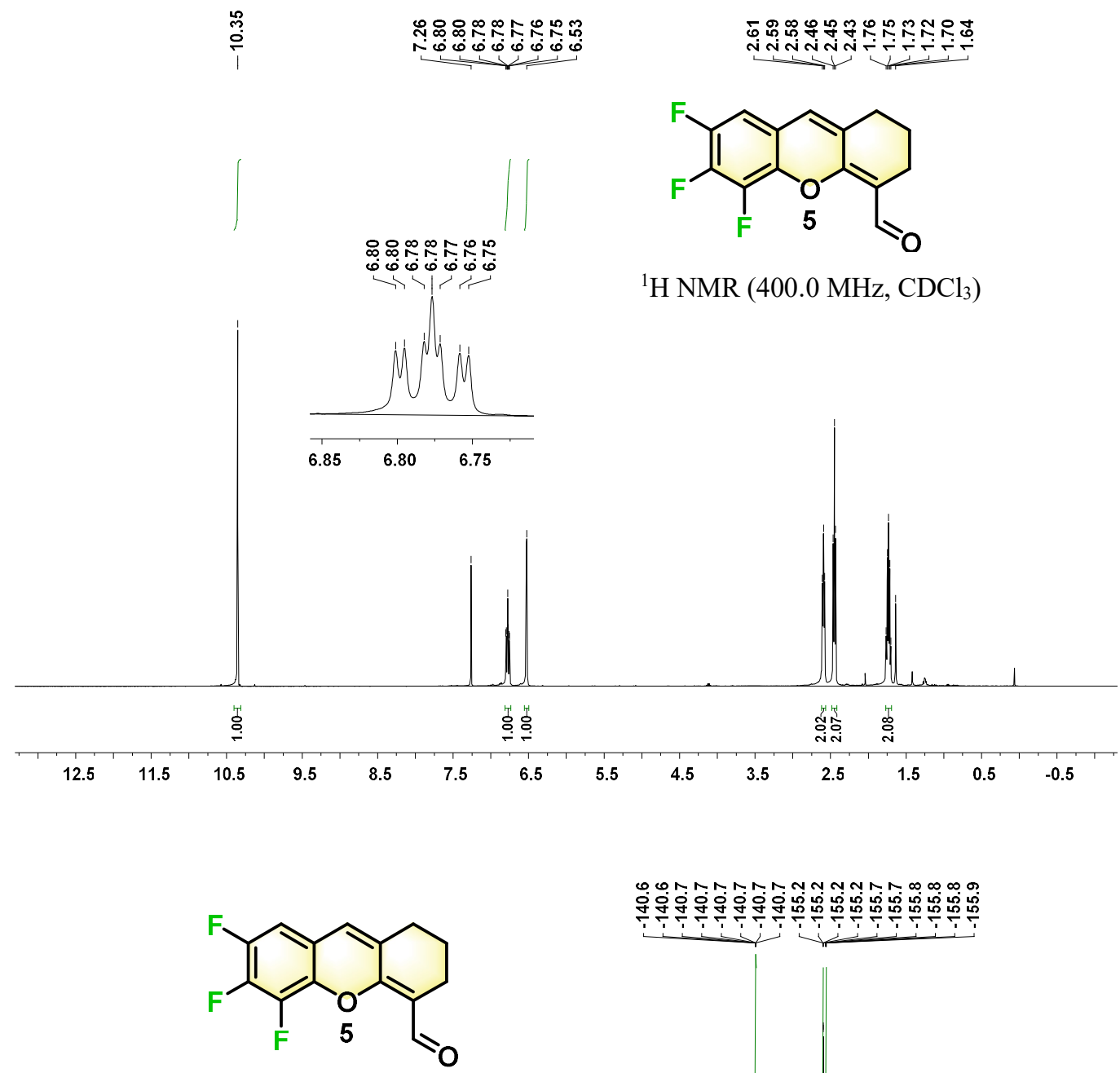

${ }^{19} \mathrm{~F} \mathrm{NMR}\left(376.5 \mathrm{MHz}, \mathrm{CDCl}_{3}\right)$

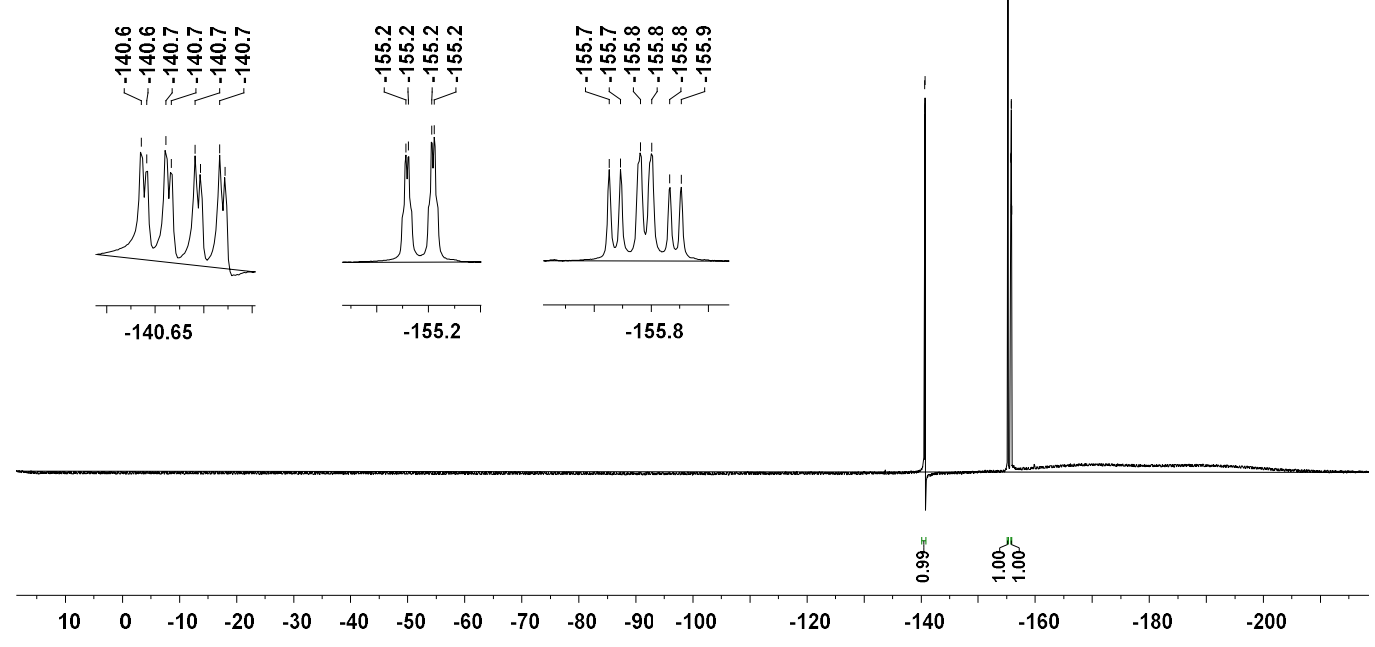




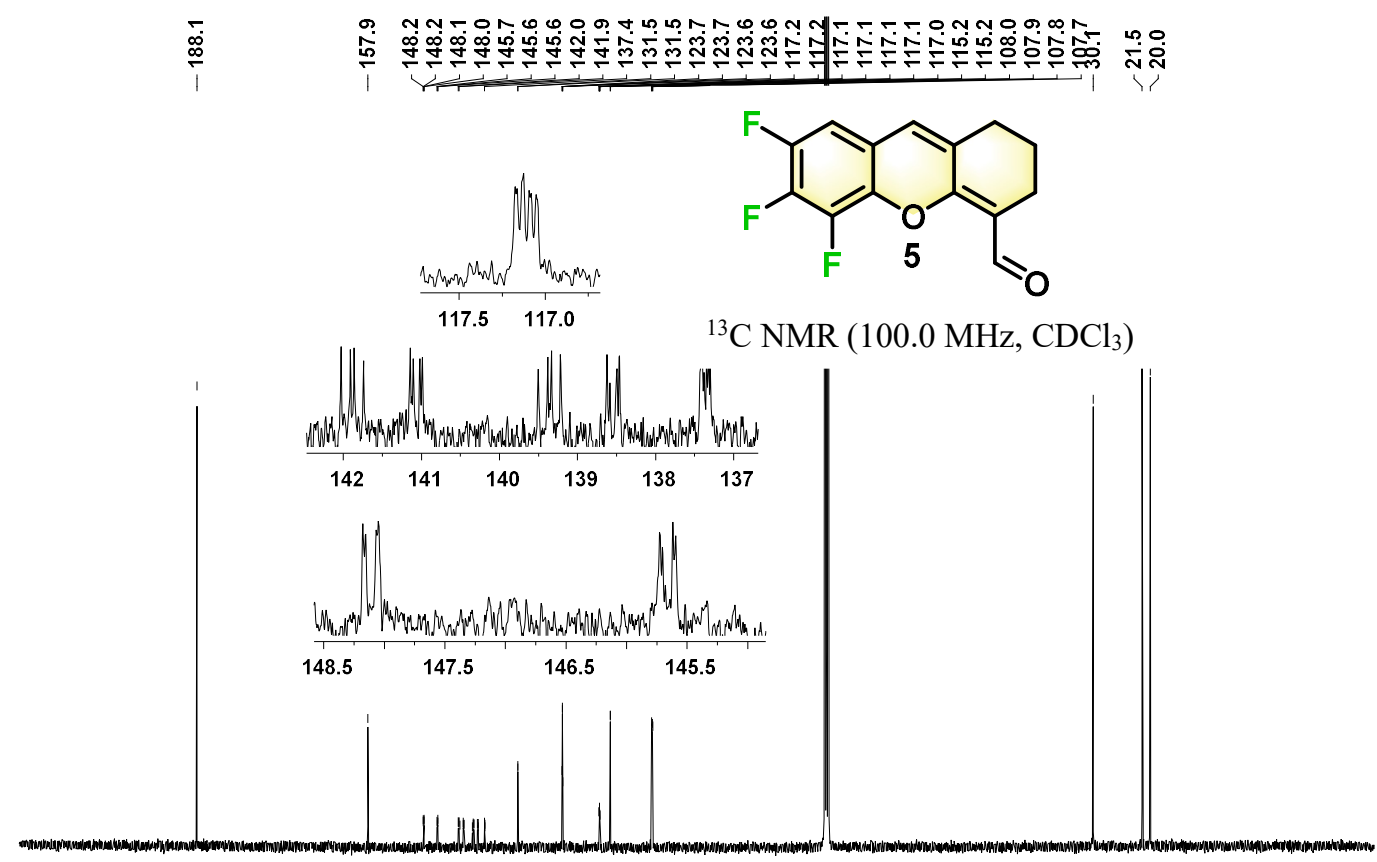

$\begin{array}{lllllllllllllllllllllll}210 & 200 & 190 & 180 & 170 & 160 & 150 & 140 & 130 & 120 & 110 & 100 & 90 & 80 & 70 & 60 & 50 & 40 & 30 & 20 & 10 & 0 & -10\end{array}$

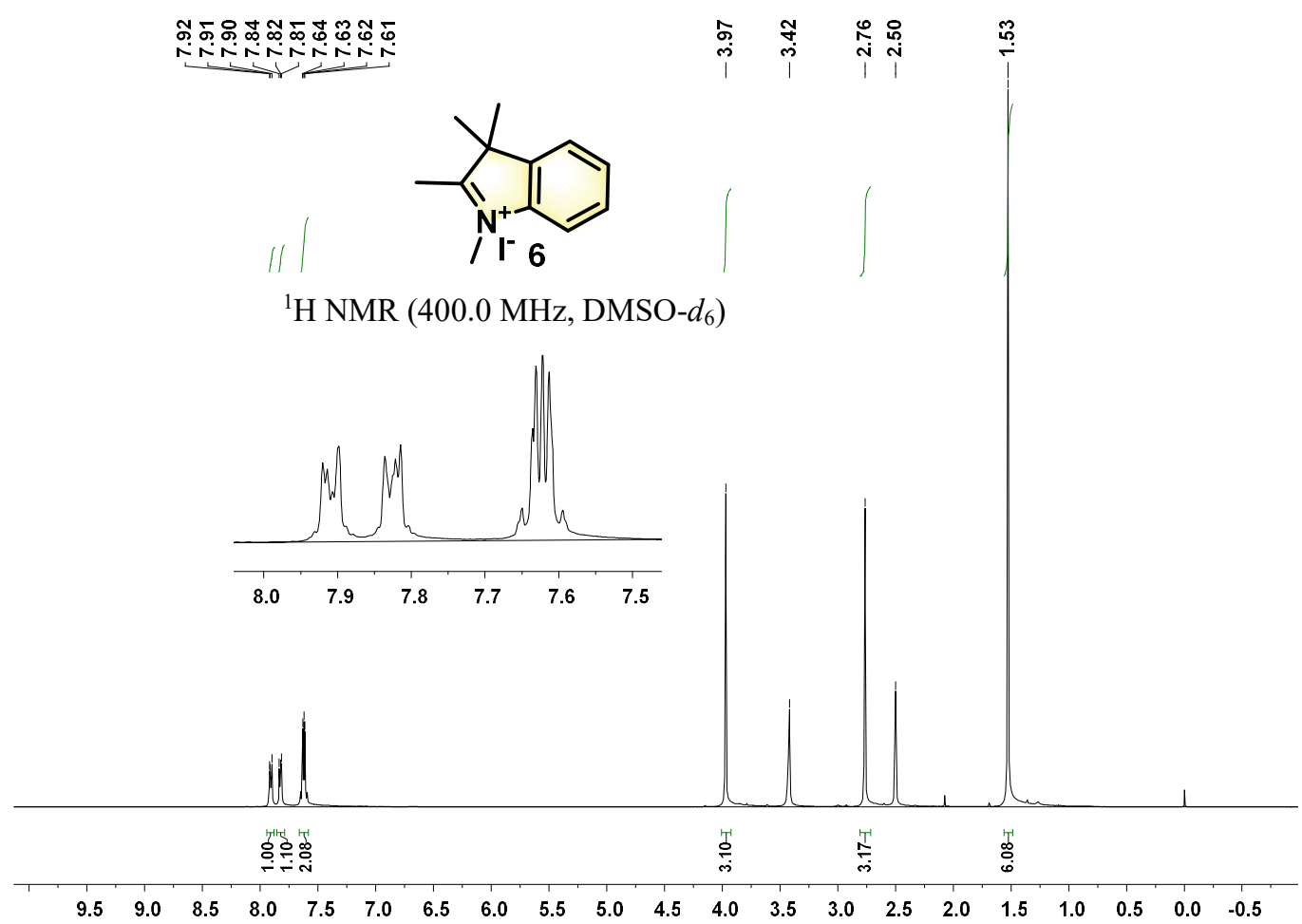


<smiles>CC1=[N+](C)c2ccccc2C1(C)C</smiles>

${ }^{13} \mathrm{C}$ NMR (100.0 MHz, DMSO- $\left.d_{6}\right)$

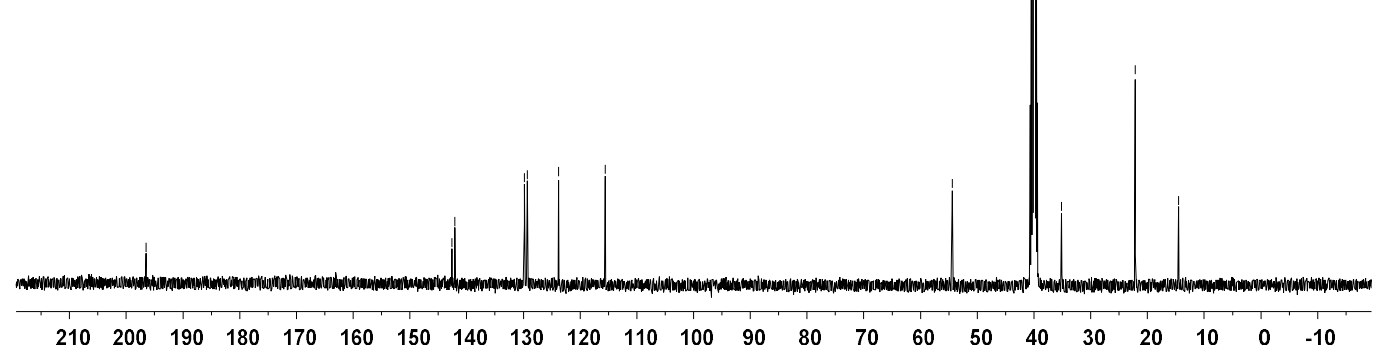

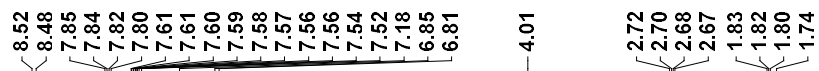<smiles></smiles>

${ }^{1} \mathrm{H}$ NMR (400.0 MHz, DMSO- $d_{6}$ )

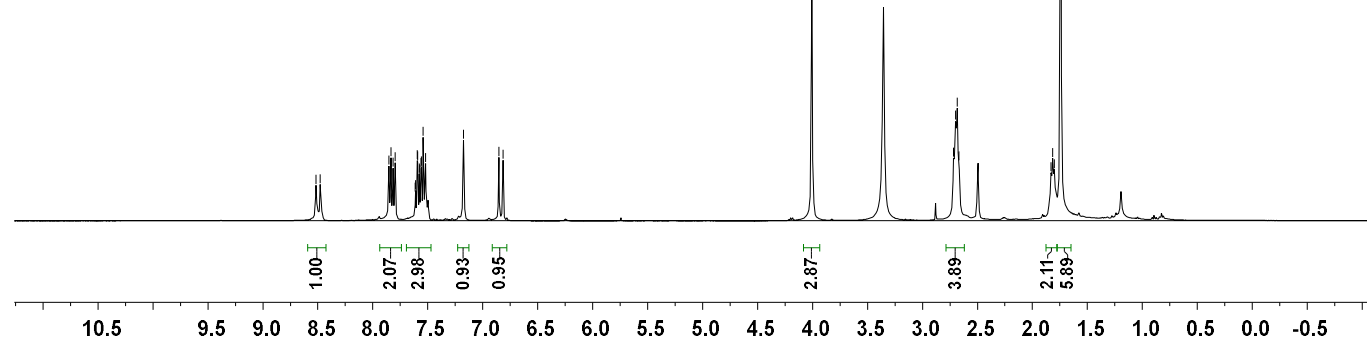



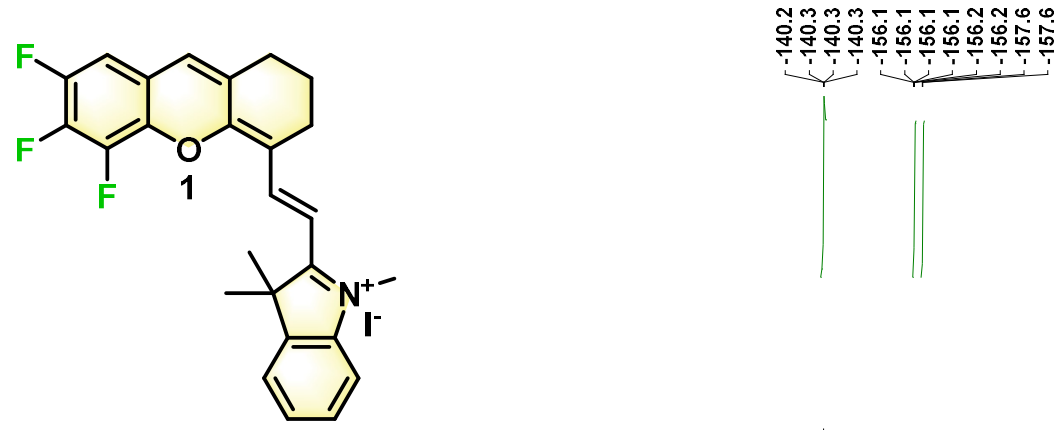

${ }^{19}$ F NMR (376.5 MHz, DMSO- $\left.d_{6}\right)$

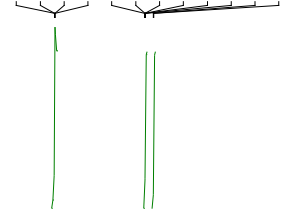

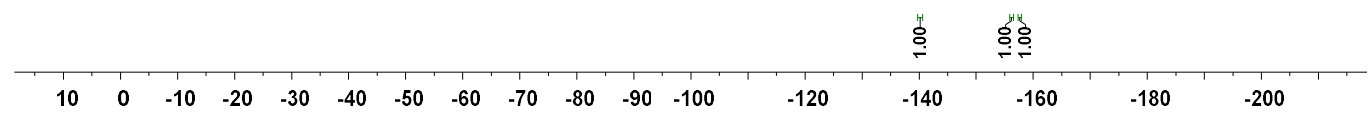
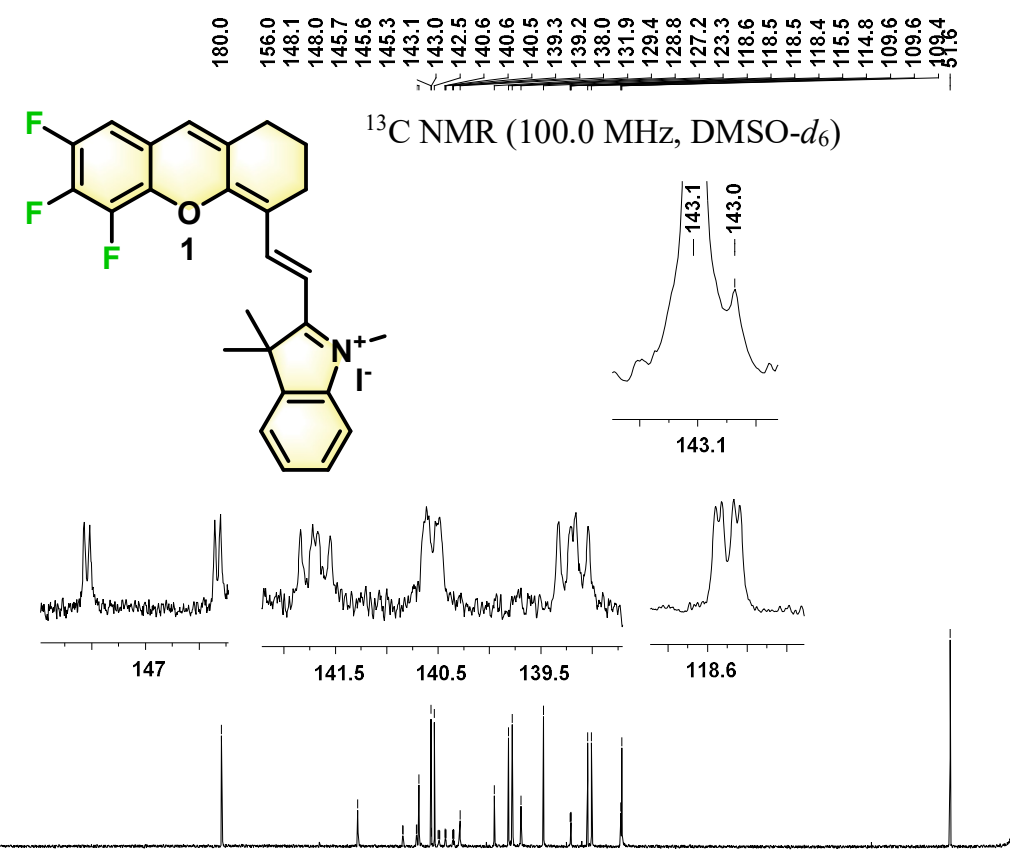

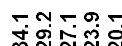

। 1 ।

$\begin{array}{lllllllllllllllllllllll}210 & 200 & 190 & 180 & 170 & 160 & 150 & 140 & 130 & 120 & 110 & 100 & 90 & 80 & 70 & 60 & 50 & 40 & 30 & 20 & 10 & 0 & -10\end{array}$ 

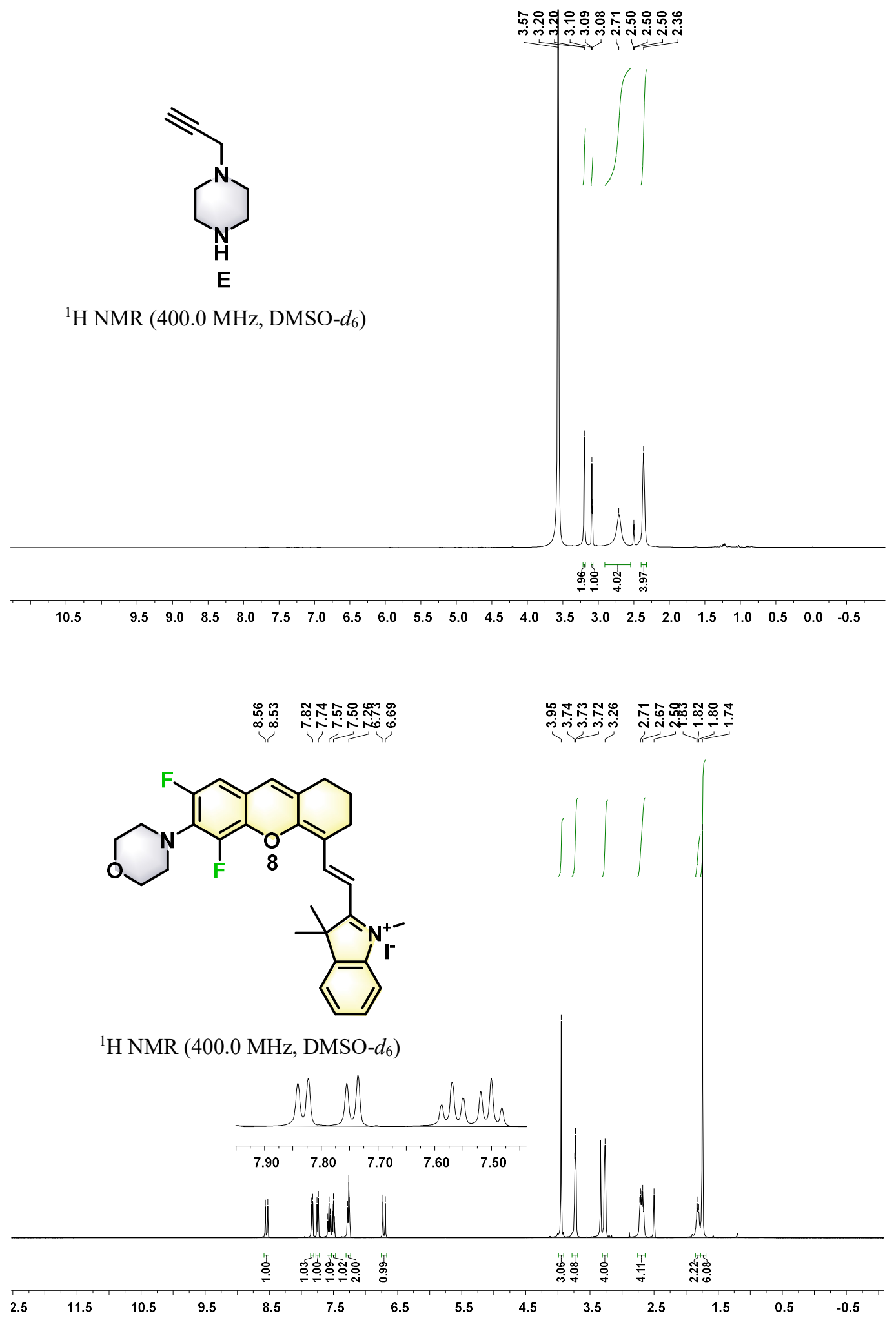

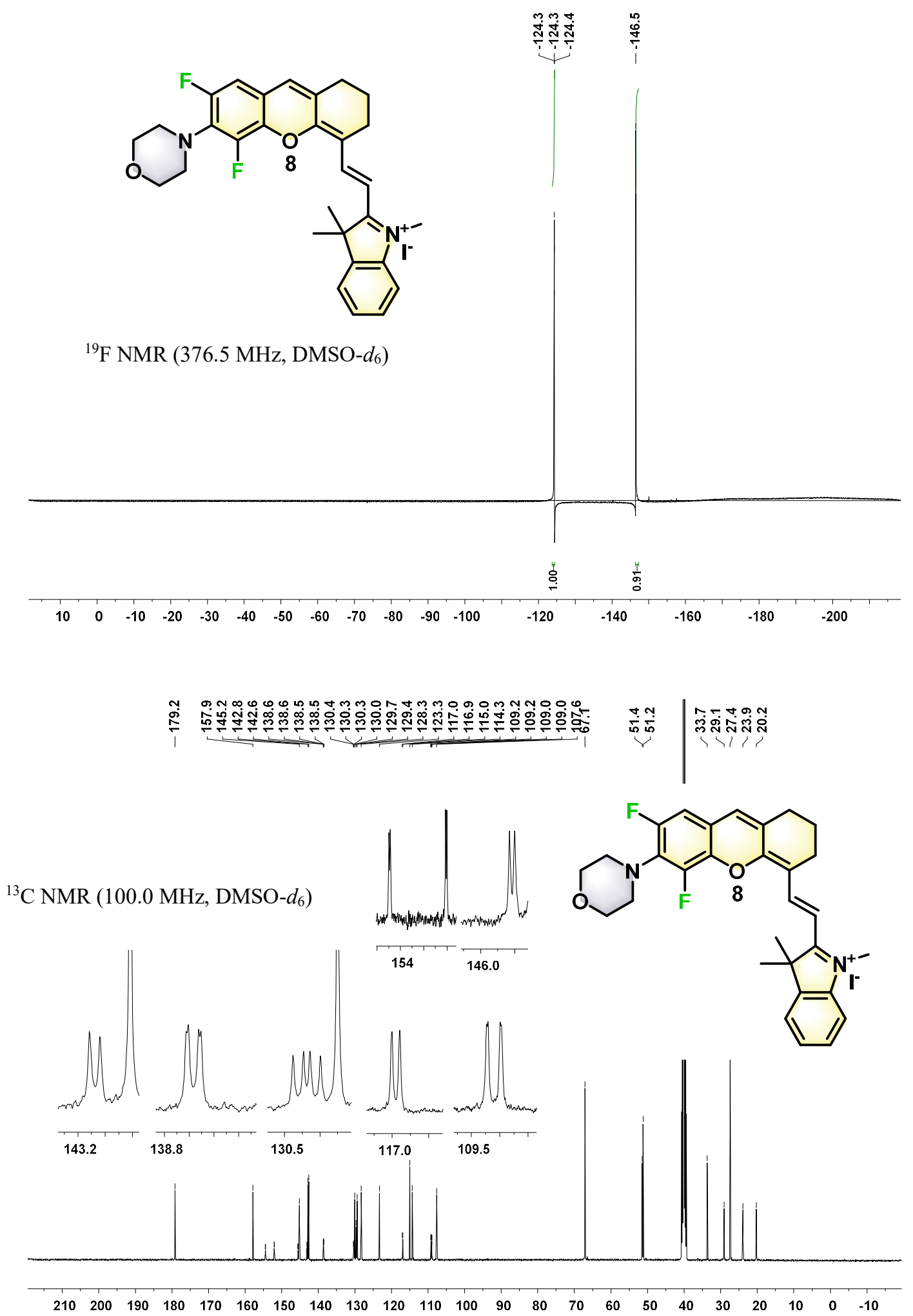

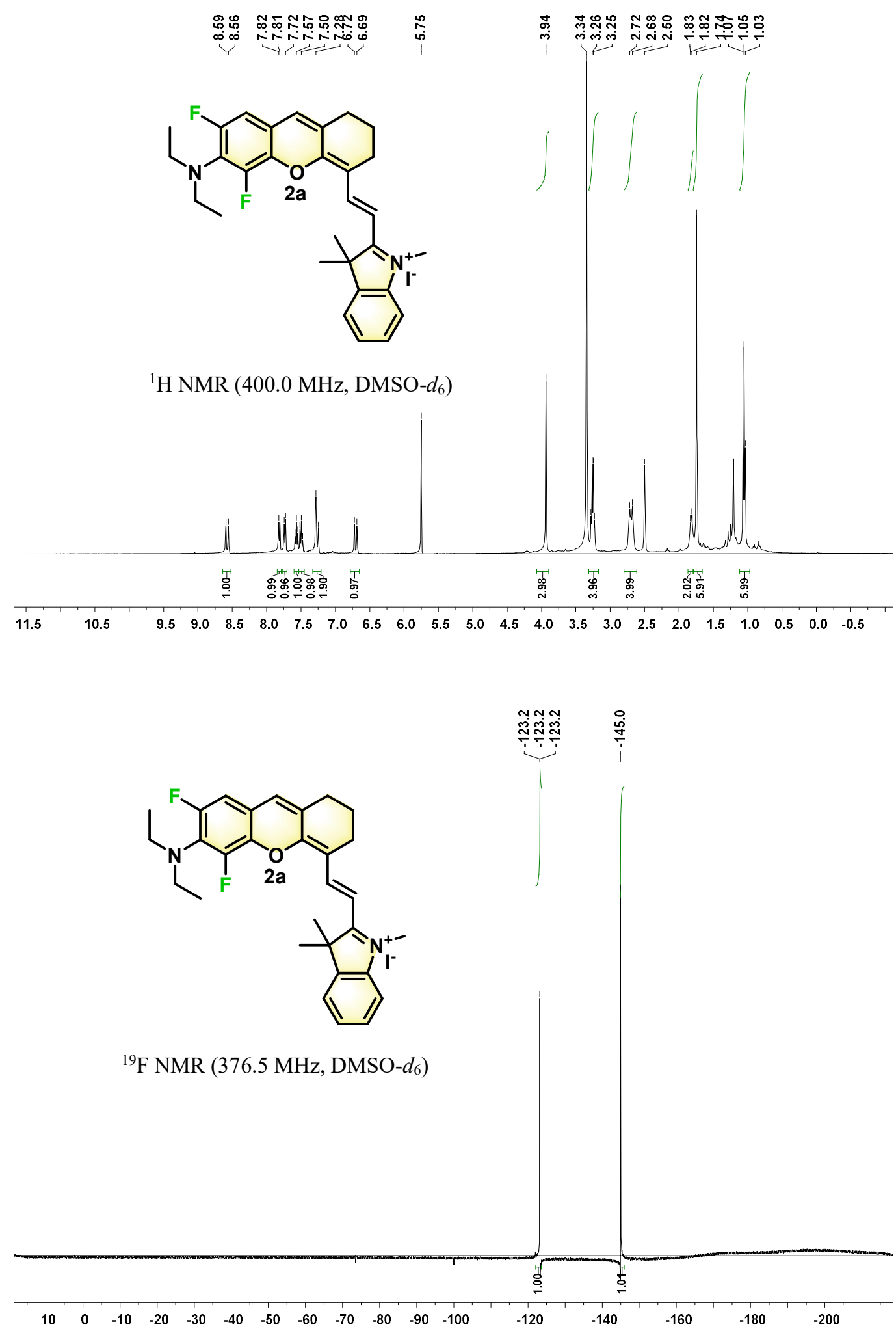


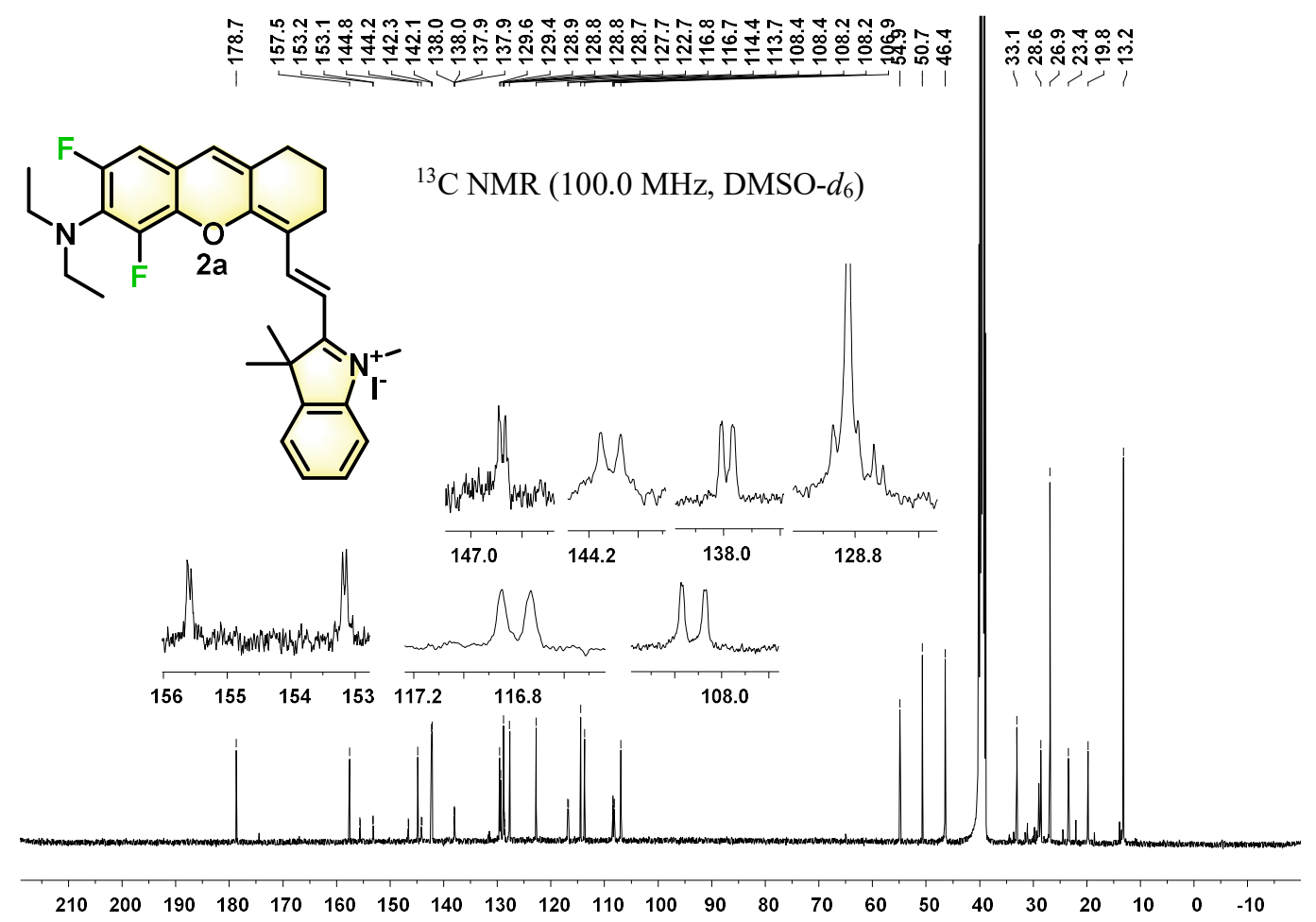




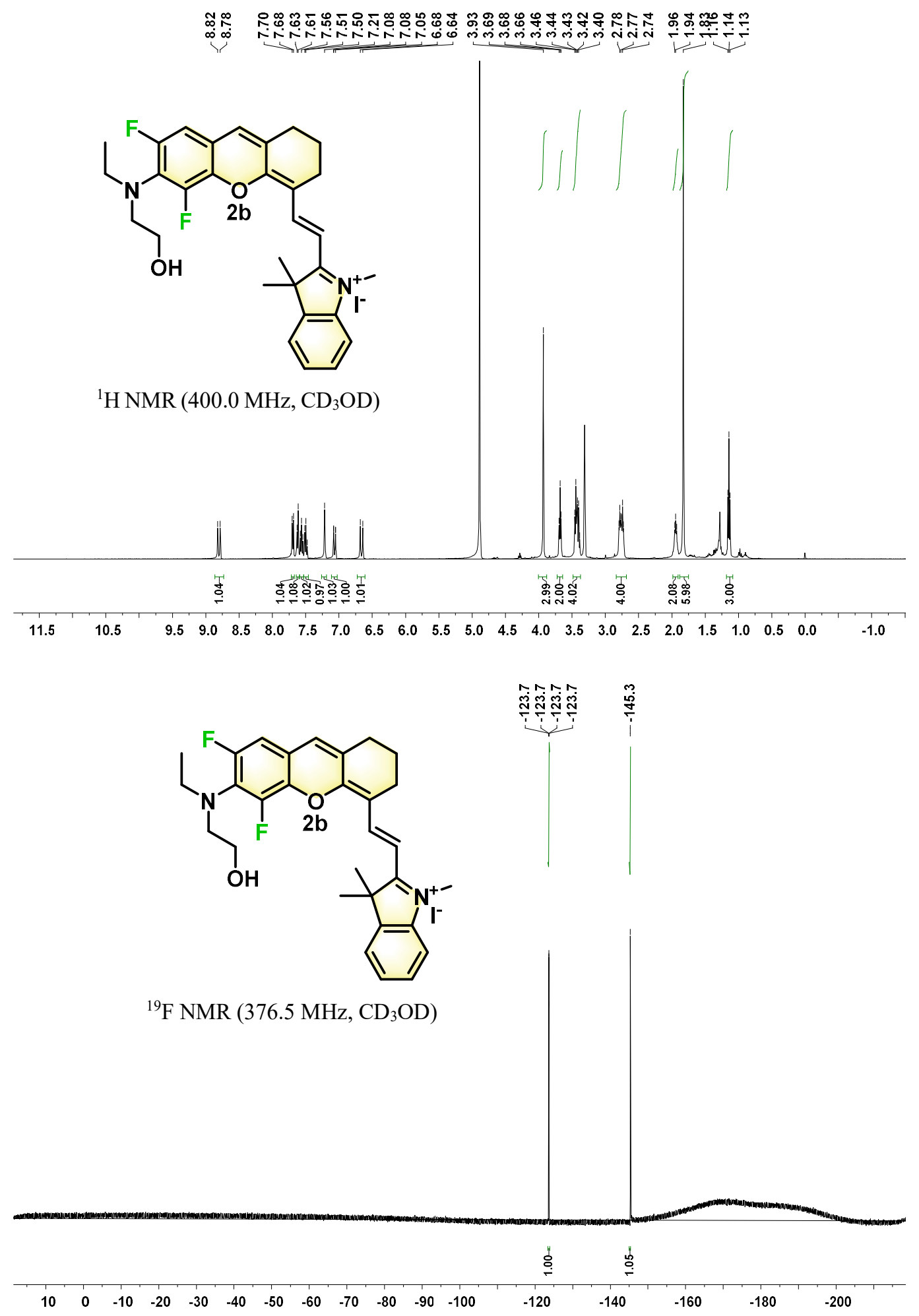




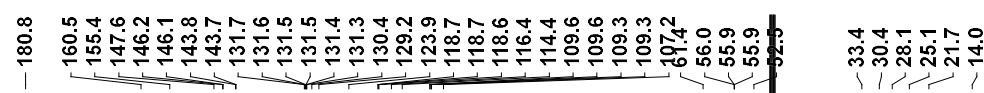

${ }^{13} \mathrm{C}$ NMR (100.0 MHz, $\left.\mathrm{CD}_{3} \mathrm{OD}\right)$

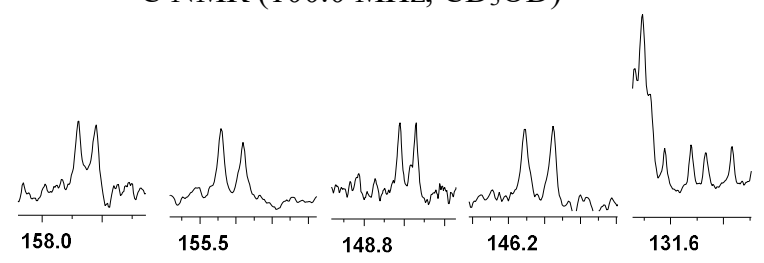

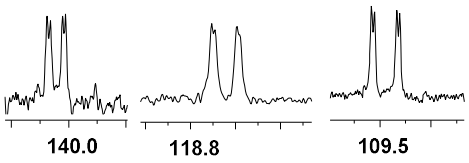

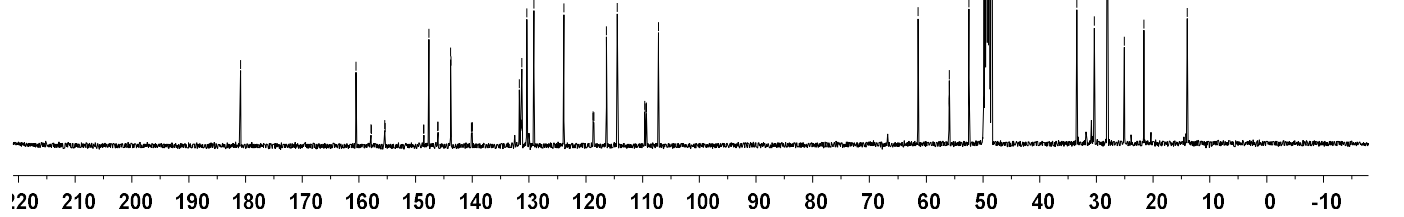

$\begin{array}{llllllllllllllllllllllll}: 20 & 210 & 200 & 190 & 180 & 170 & 160 & 150 & 140 & 130 & 120 & 110 & 100 & 90 & 80 & 70 & 60 & 50 & 40 & 30 & 20 & 10 & 0 & -10\end{array}$

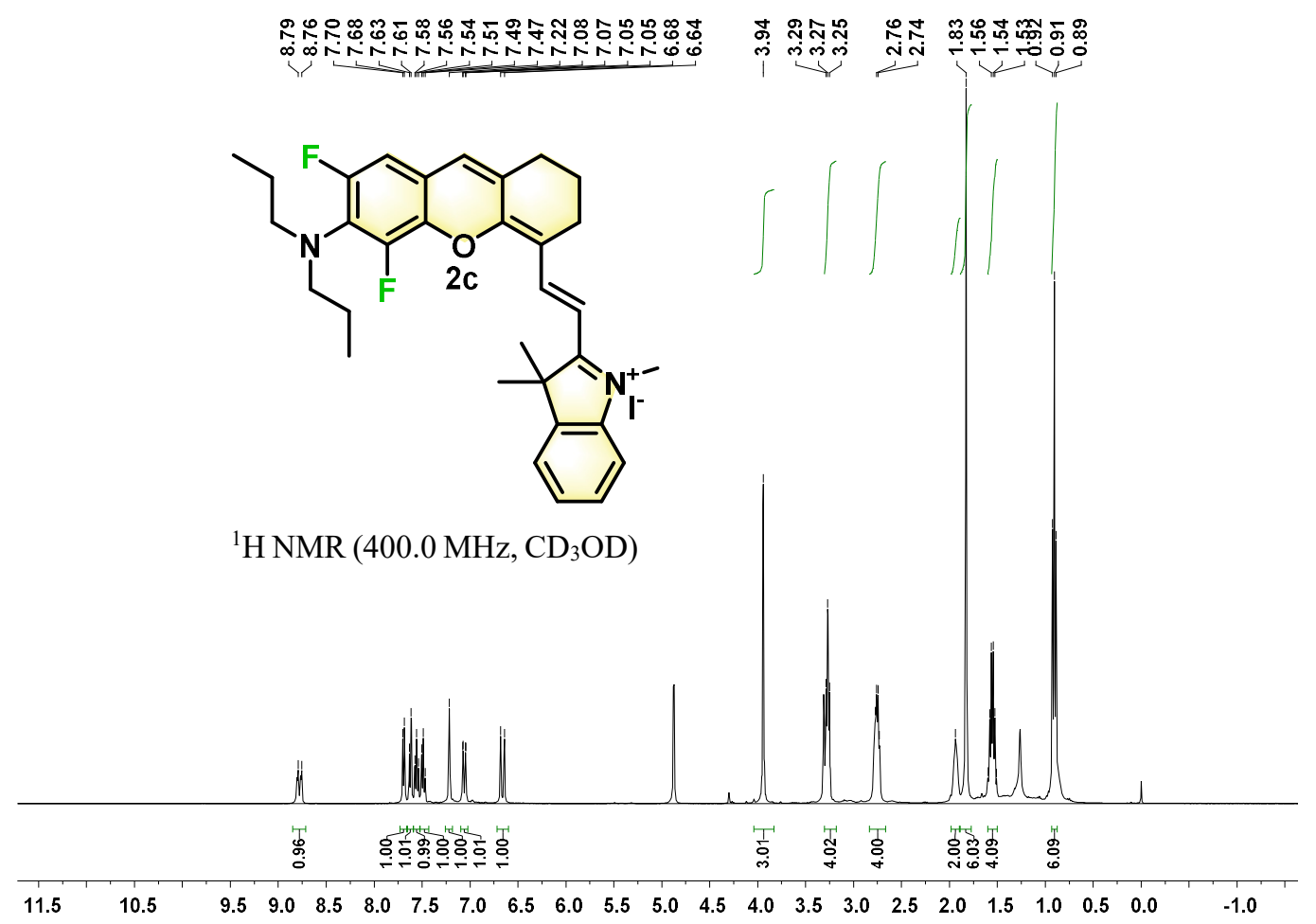




\section{穷}

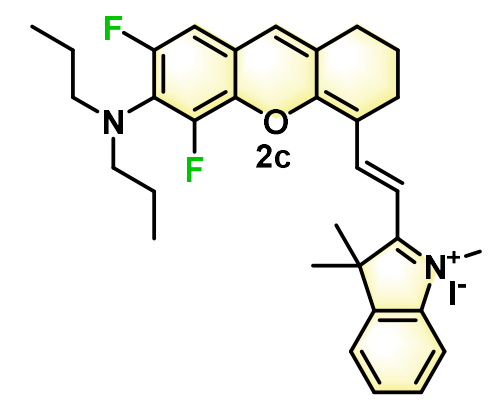

${ }^{19} \mathrm{~F}$ NMR (376.5 MHz, $\left.\mathrm{CD}_{3} \mathrm{OD}\right)$

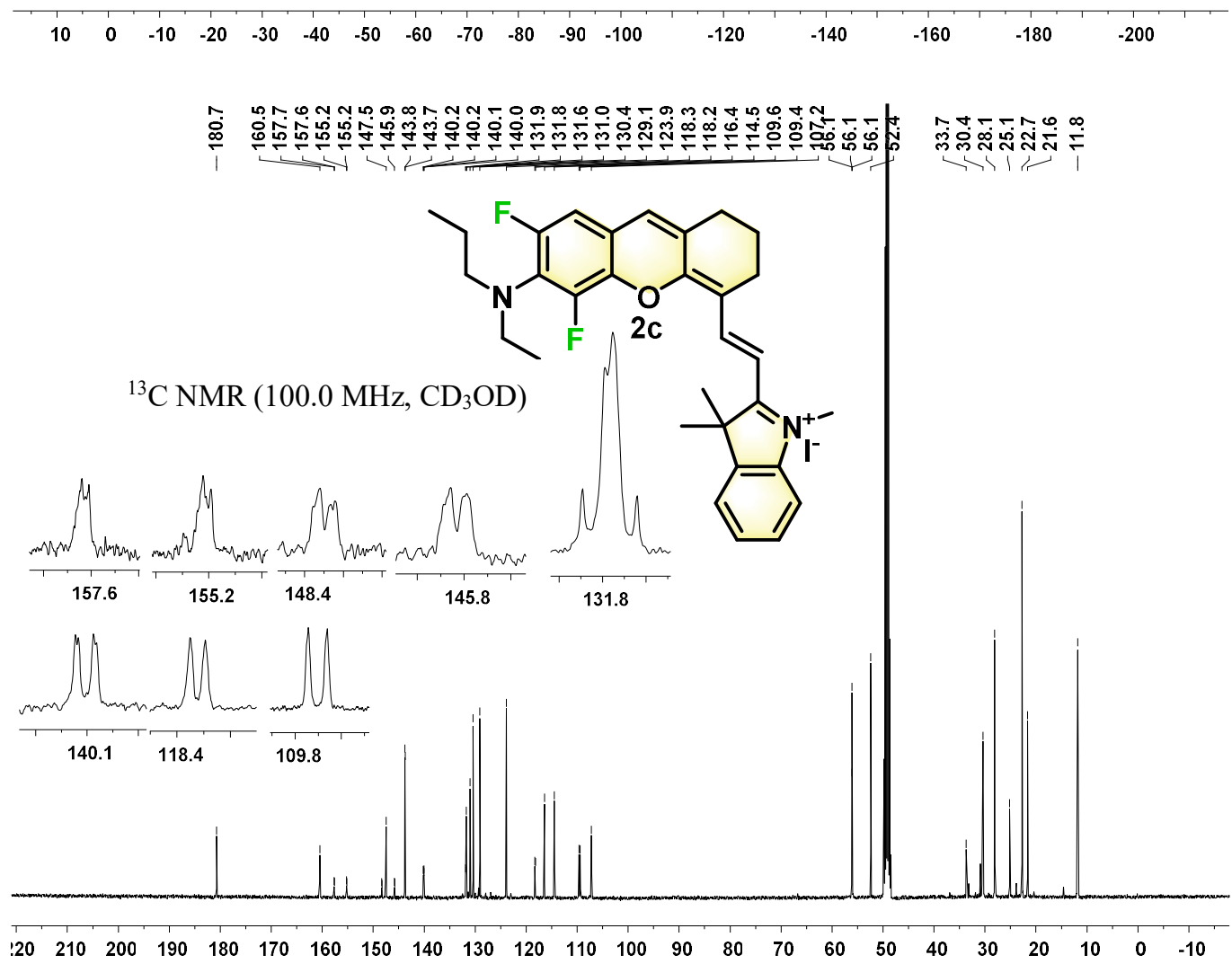



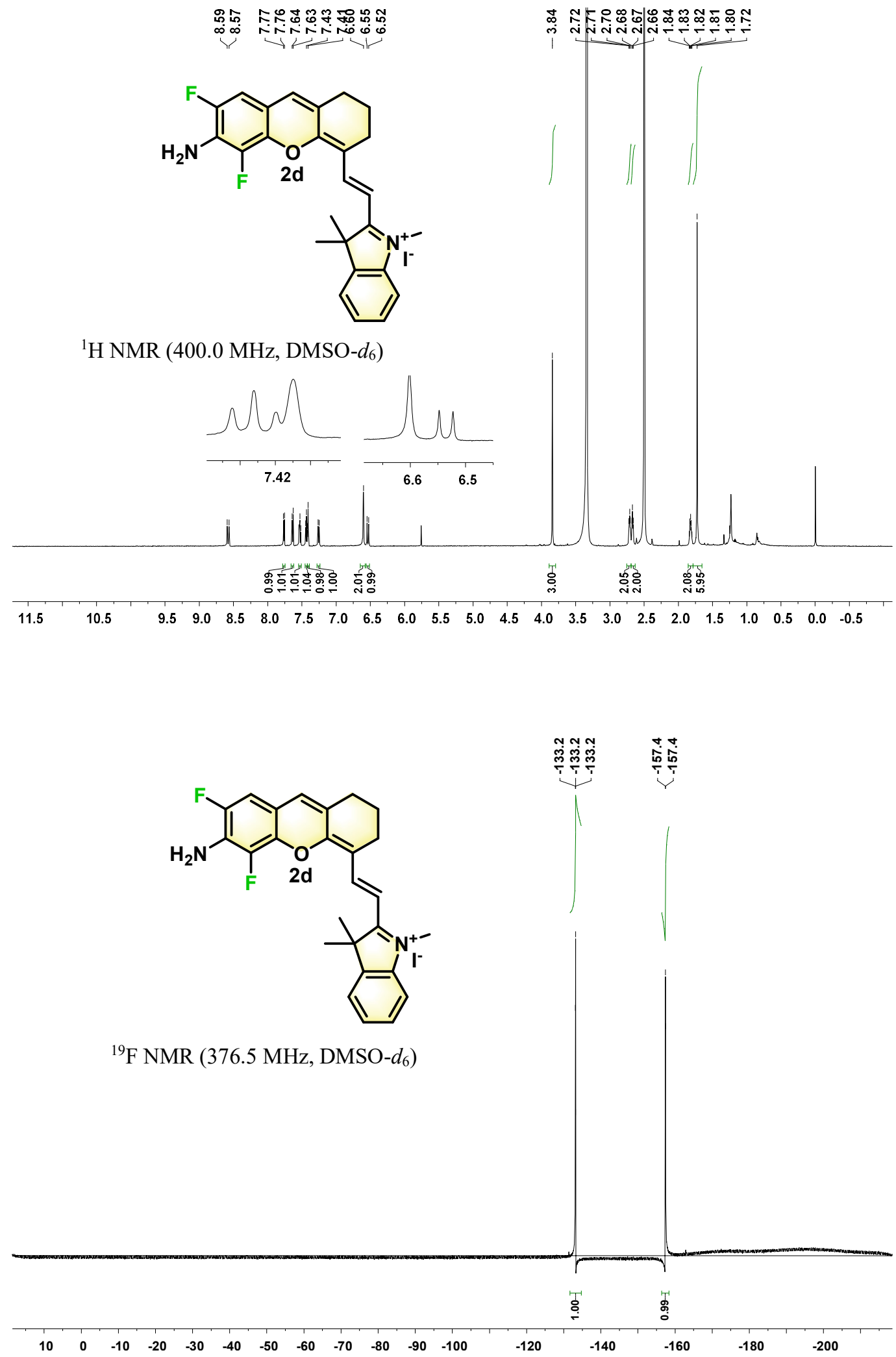


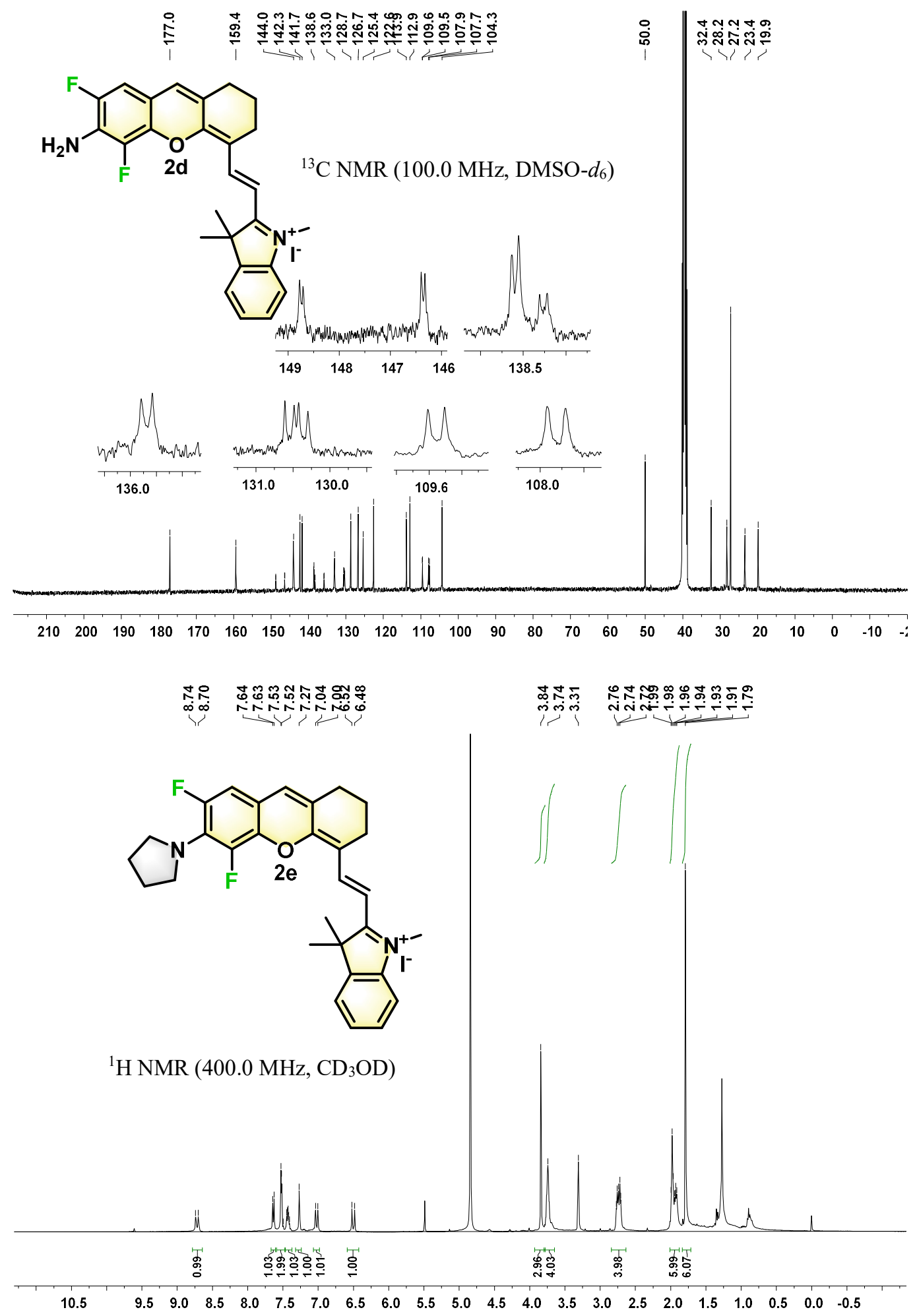




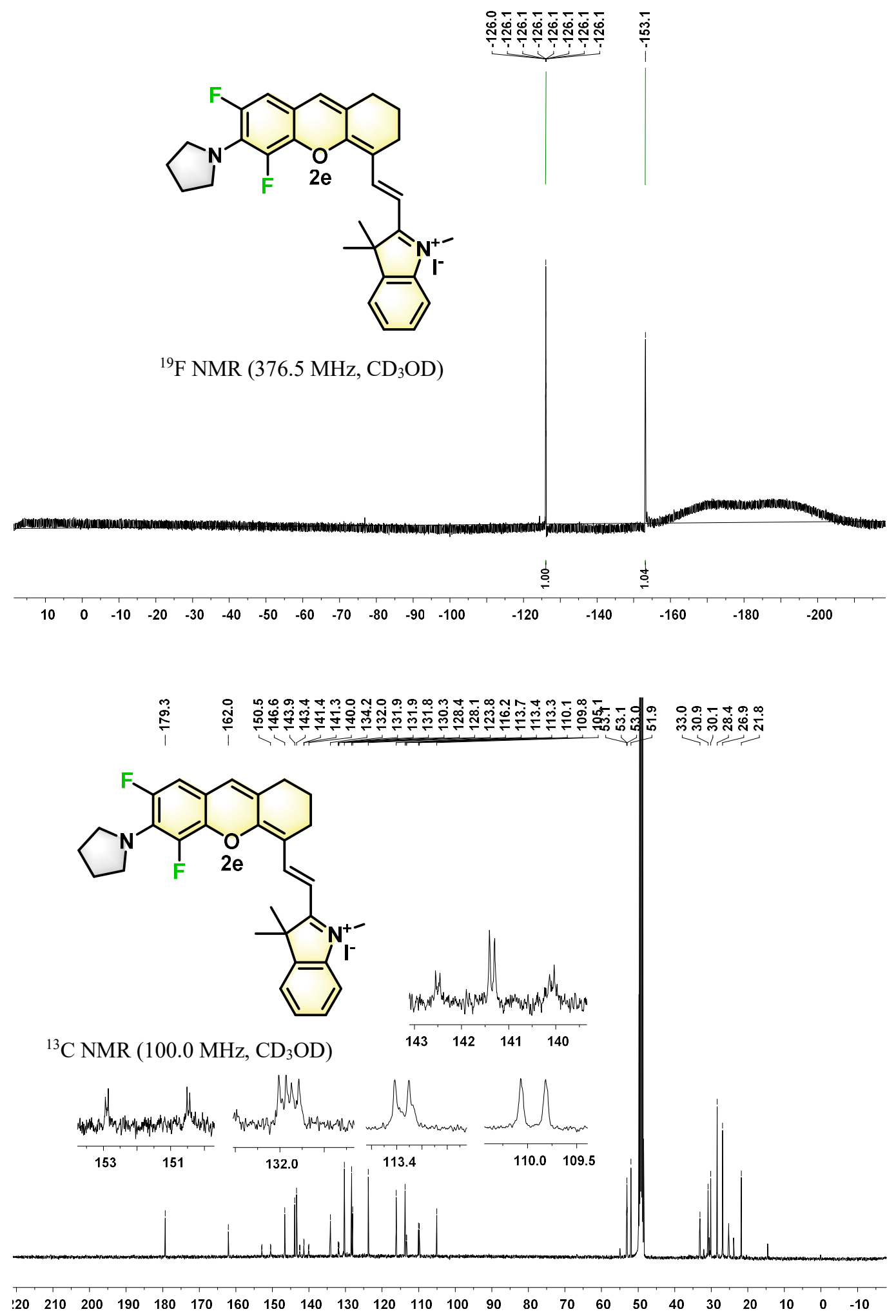




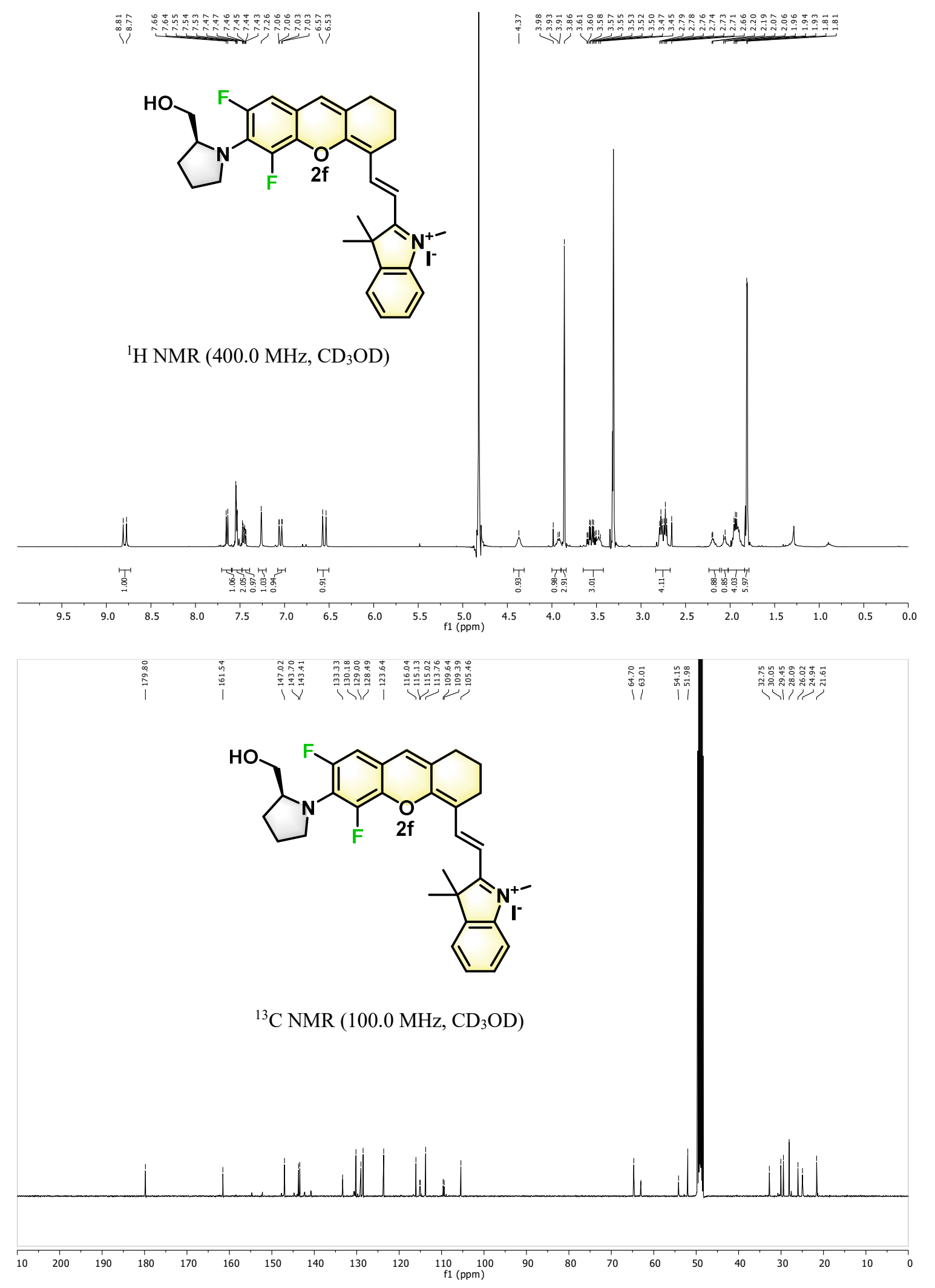




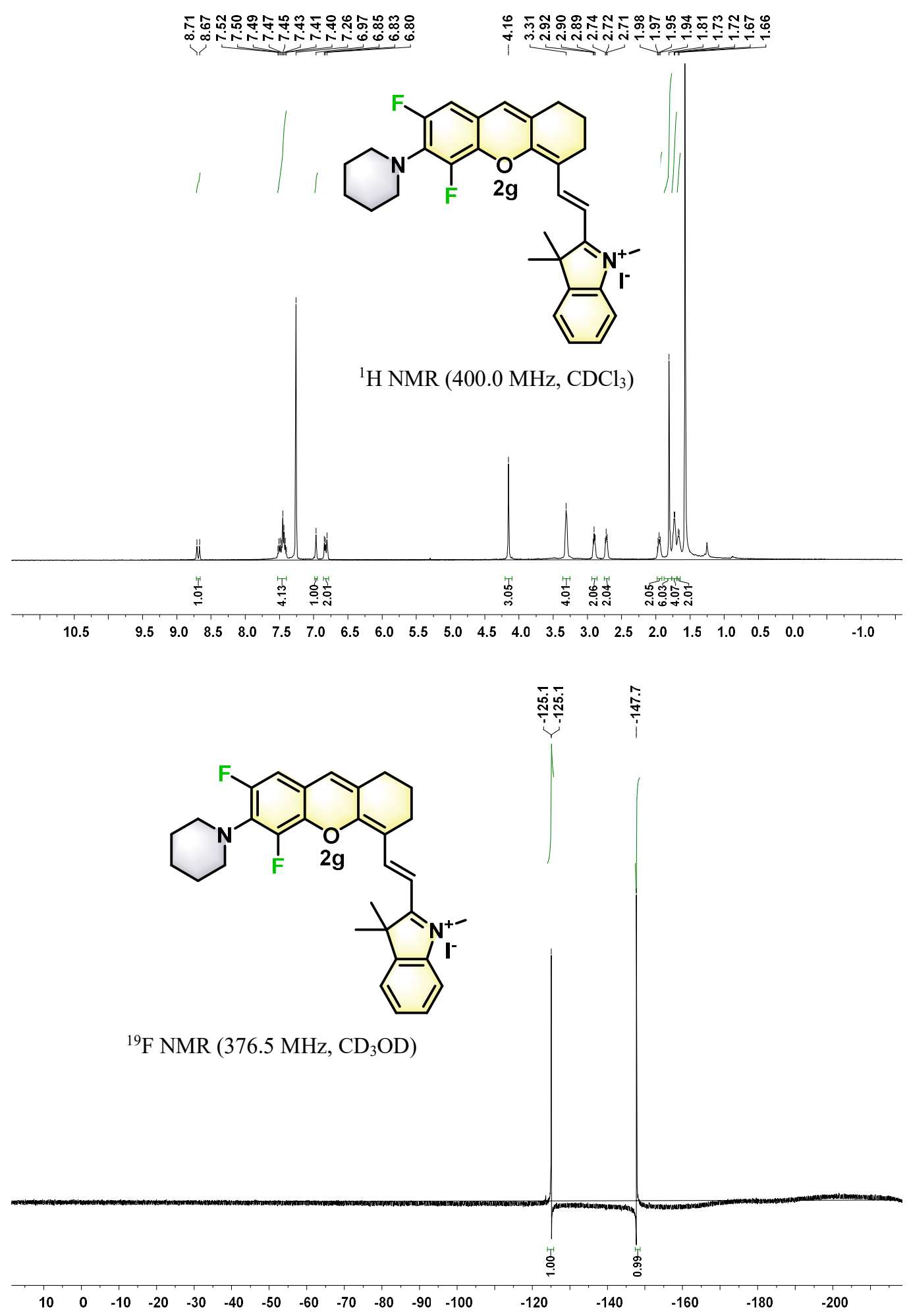



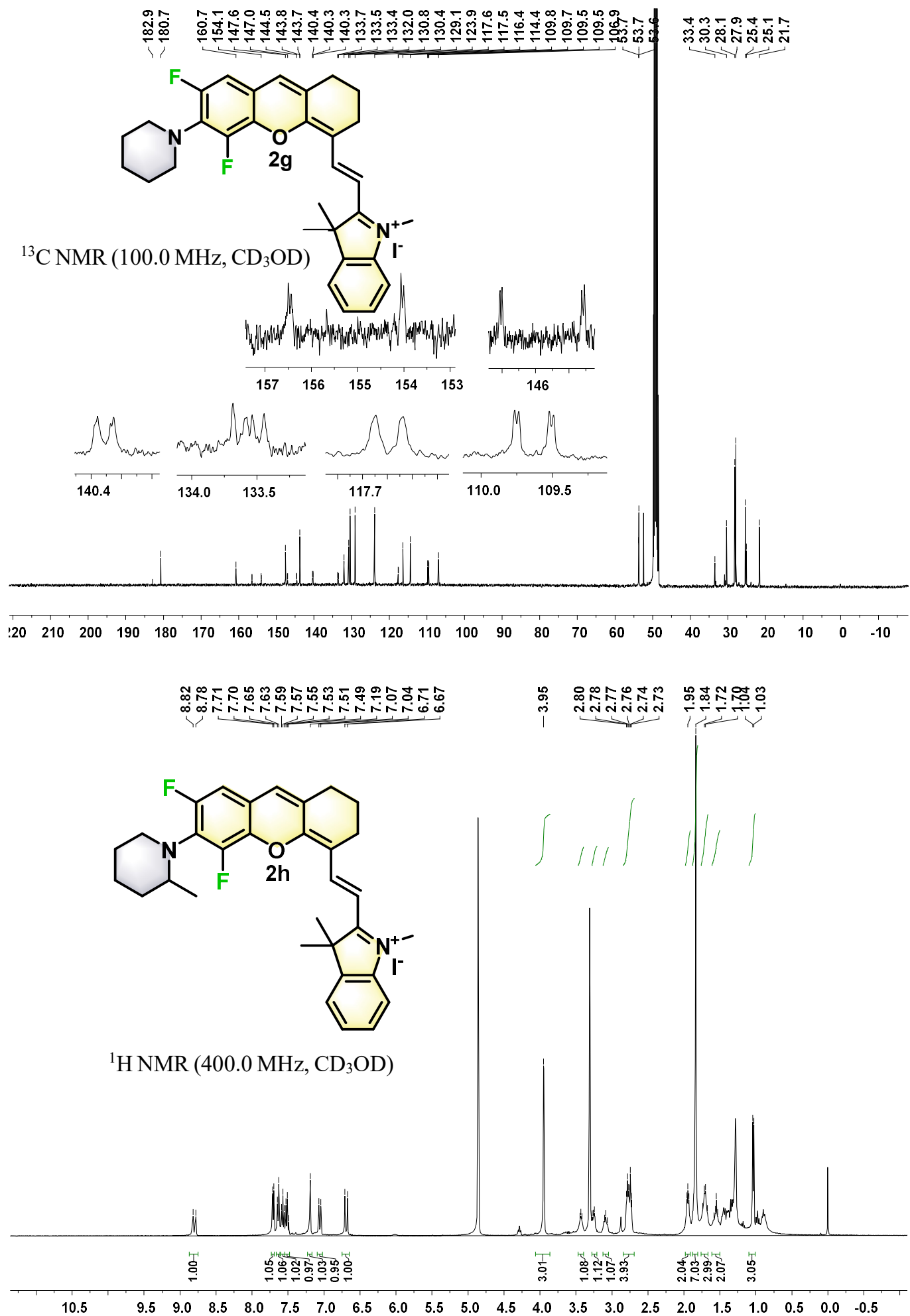

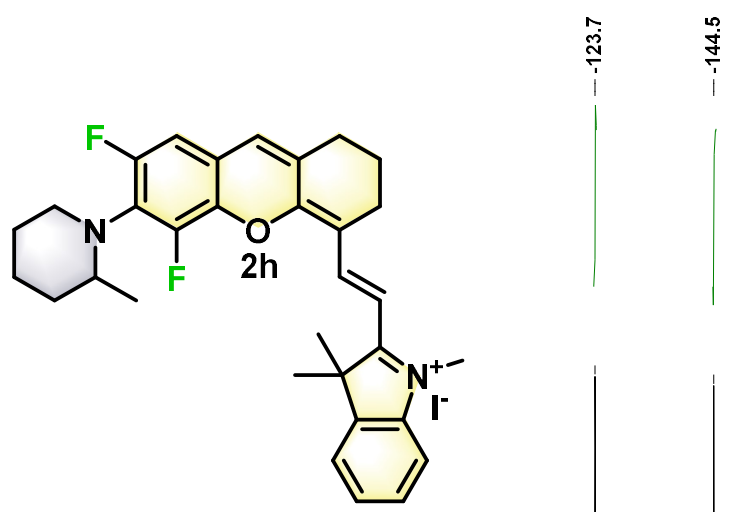

${ }^{19}$ F NMR (376.5 MHz, $\left.\mathrm{CD}_{3} \mathrm{OD}\right)$

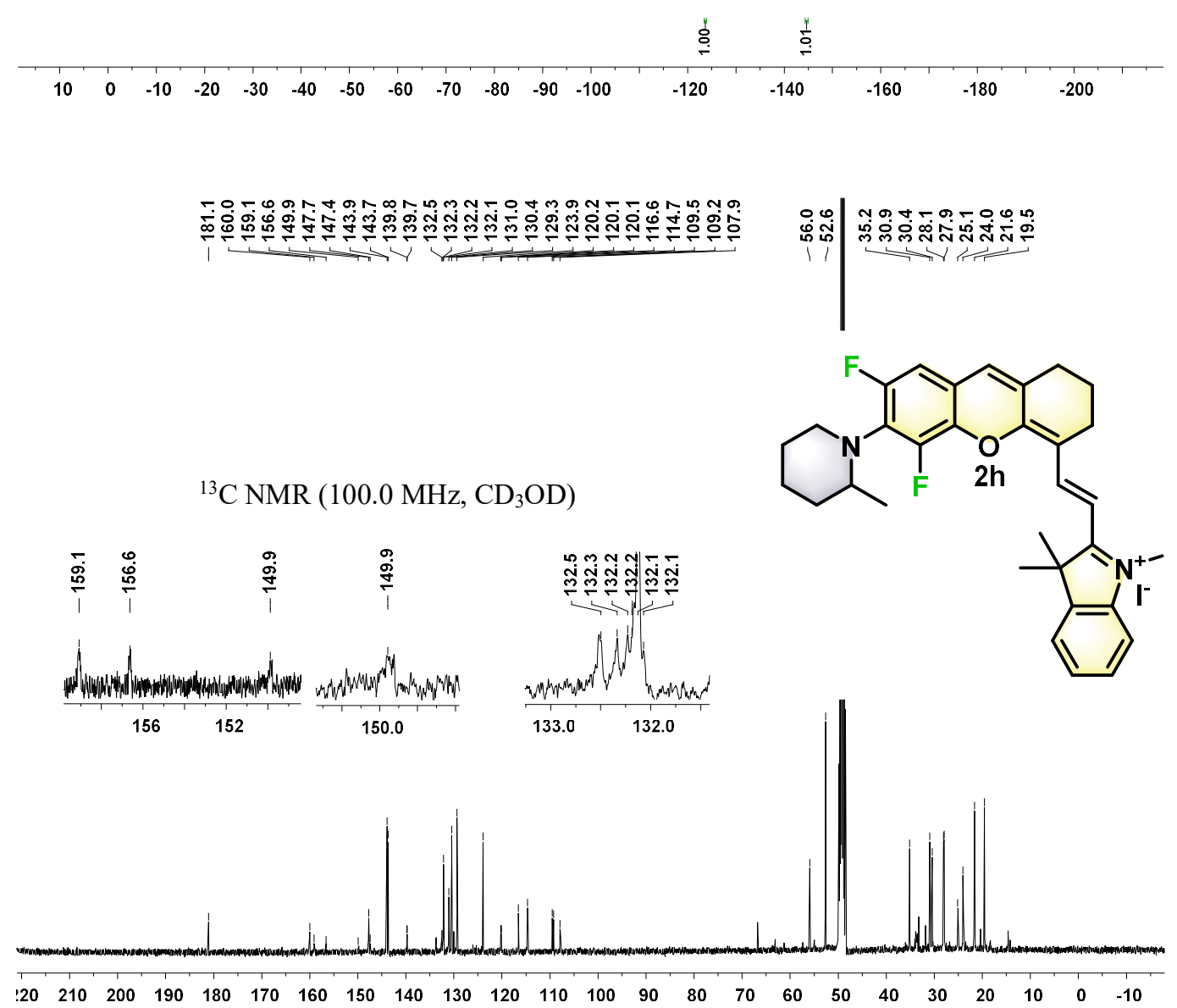




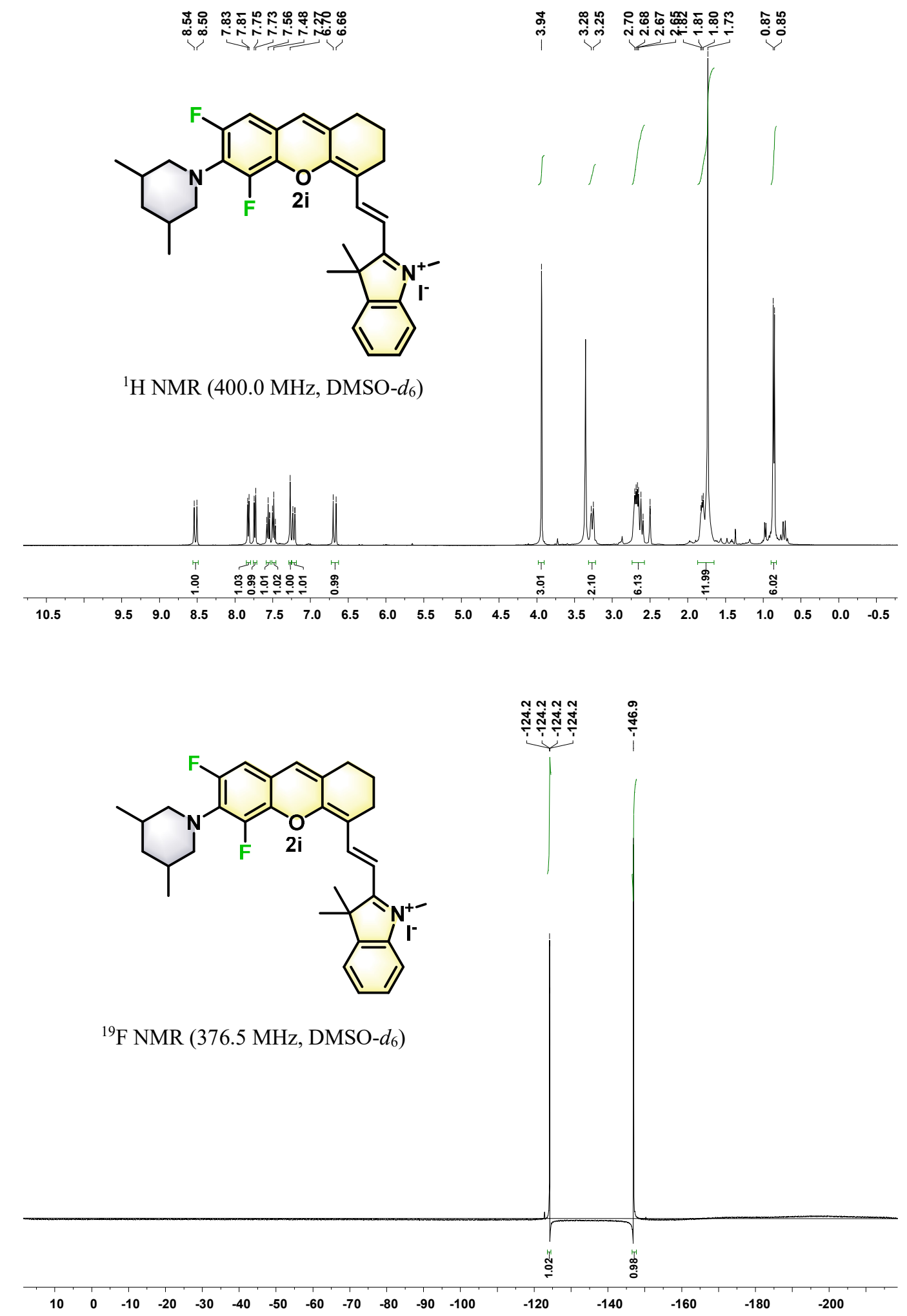



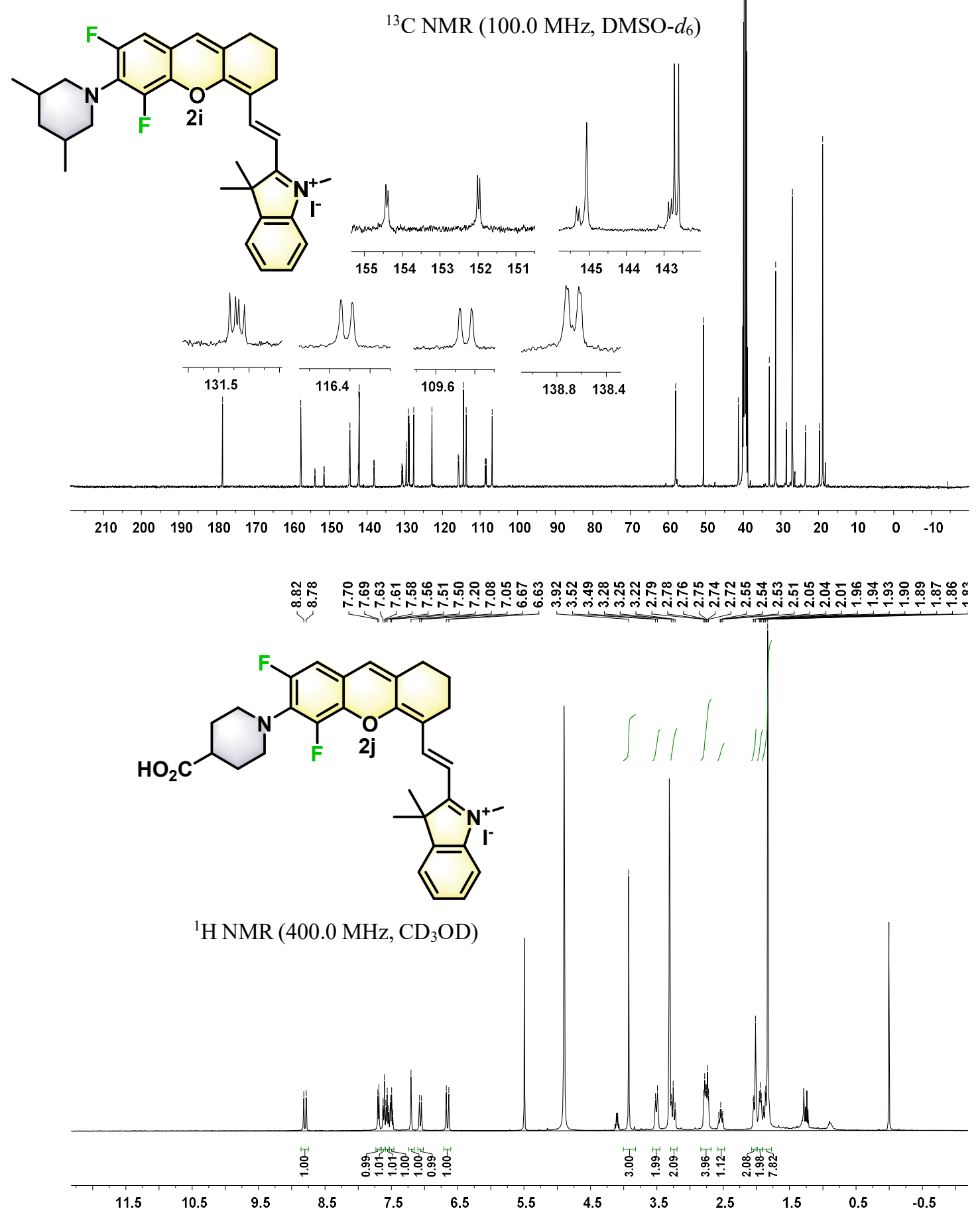


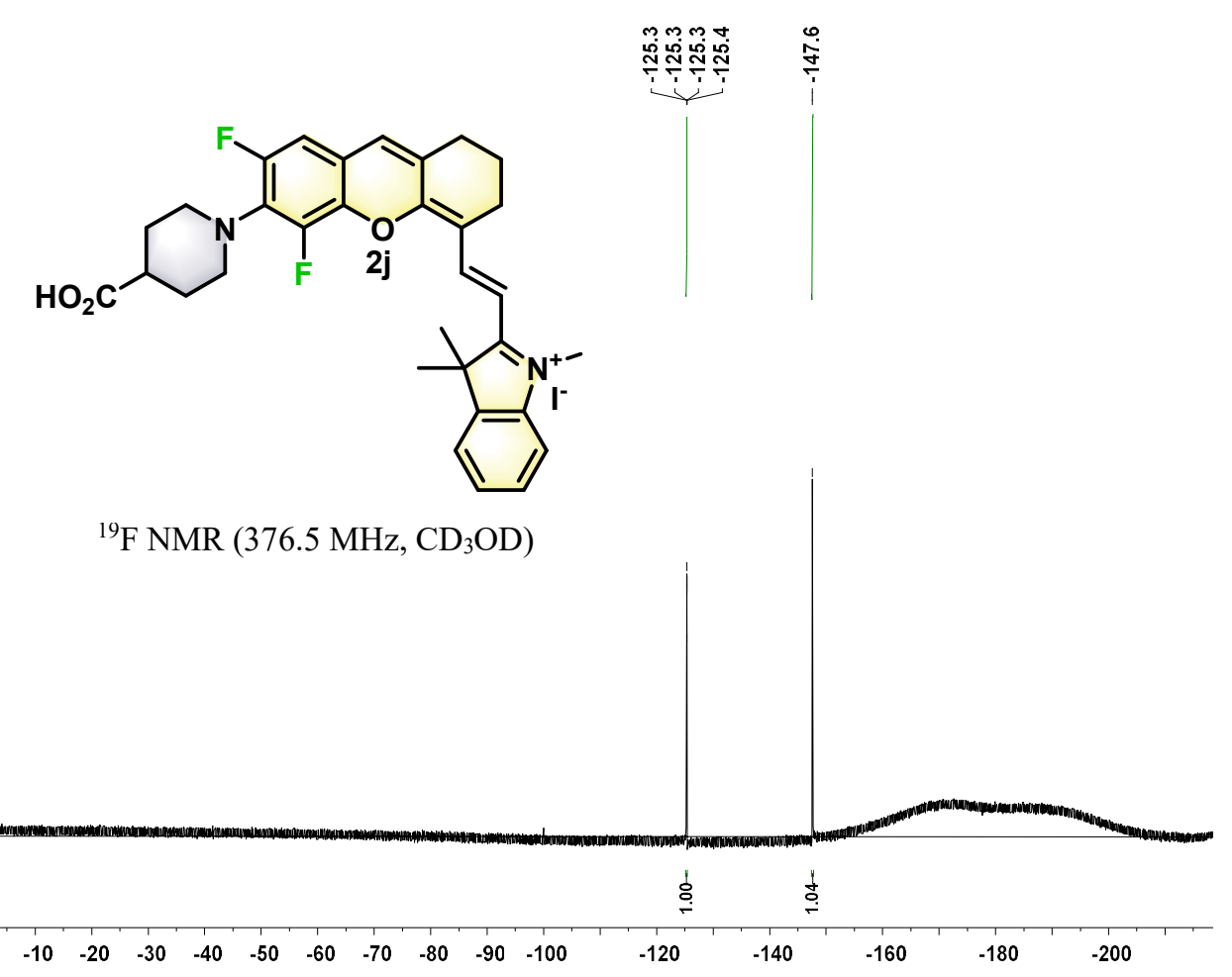

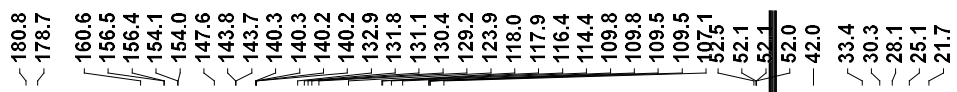<smiles>C[N+]1=C(/C=C/C2=C3Oc4c(cc(F)c(N5CCC(C(=O)O)CC5)c4F)C=C3CCC2)C(C)(C)c2ccccc21</smiles>

${ }^{13} \mathrm{C}$ NMR (100.0 MHz, $\left.\mathrm{CD}_{3} \mathrm{OD}\right)$
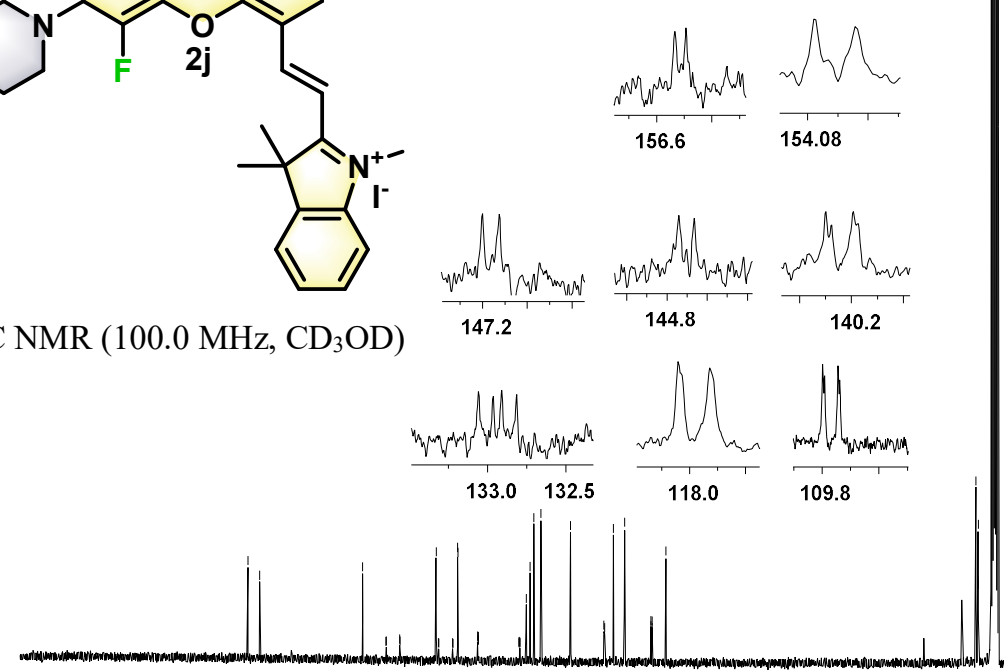

$\begin{array}{lll}147.2 & 144.8 & 140.2\end{array}$

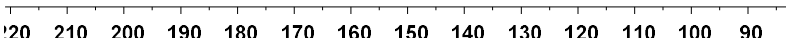

$\begin{array}{llll}80 & 70 \quad 60 & 50\end{array}$

$\begin{array}{llllll}40 & 30 & 20 & 10 & 0 & -10\end{array}$ 


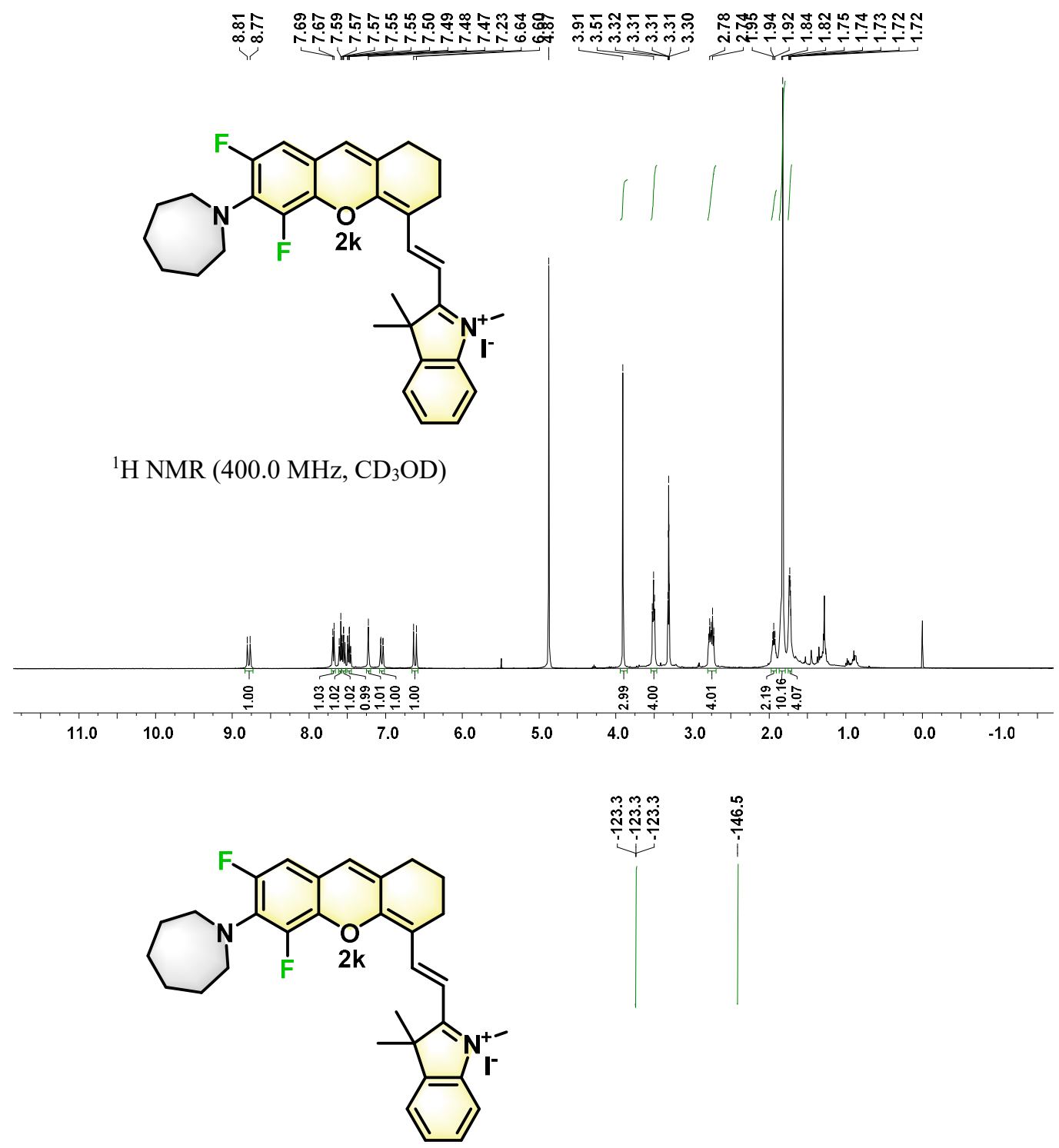

${ }^{19} \mathrm{~F}$ NMR (376.5 MHz, $\left.\mathrm{CD}_{3} \mathrm{OD}\right)$

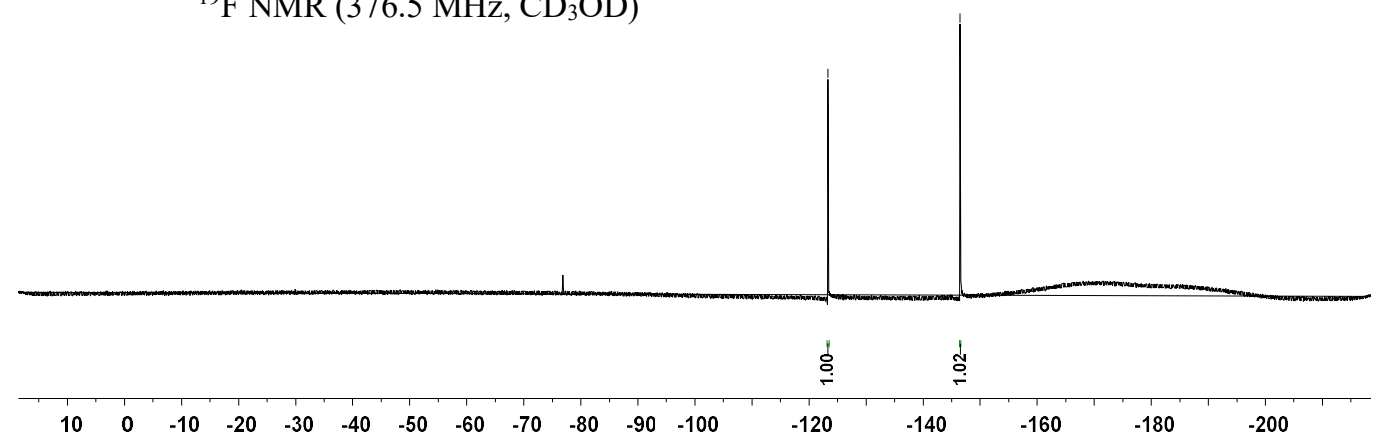



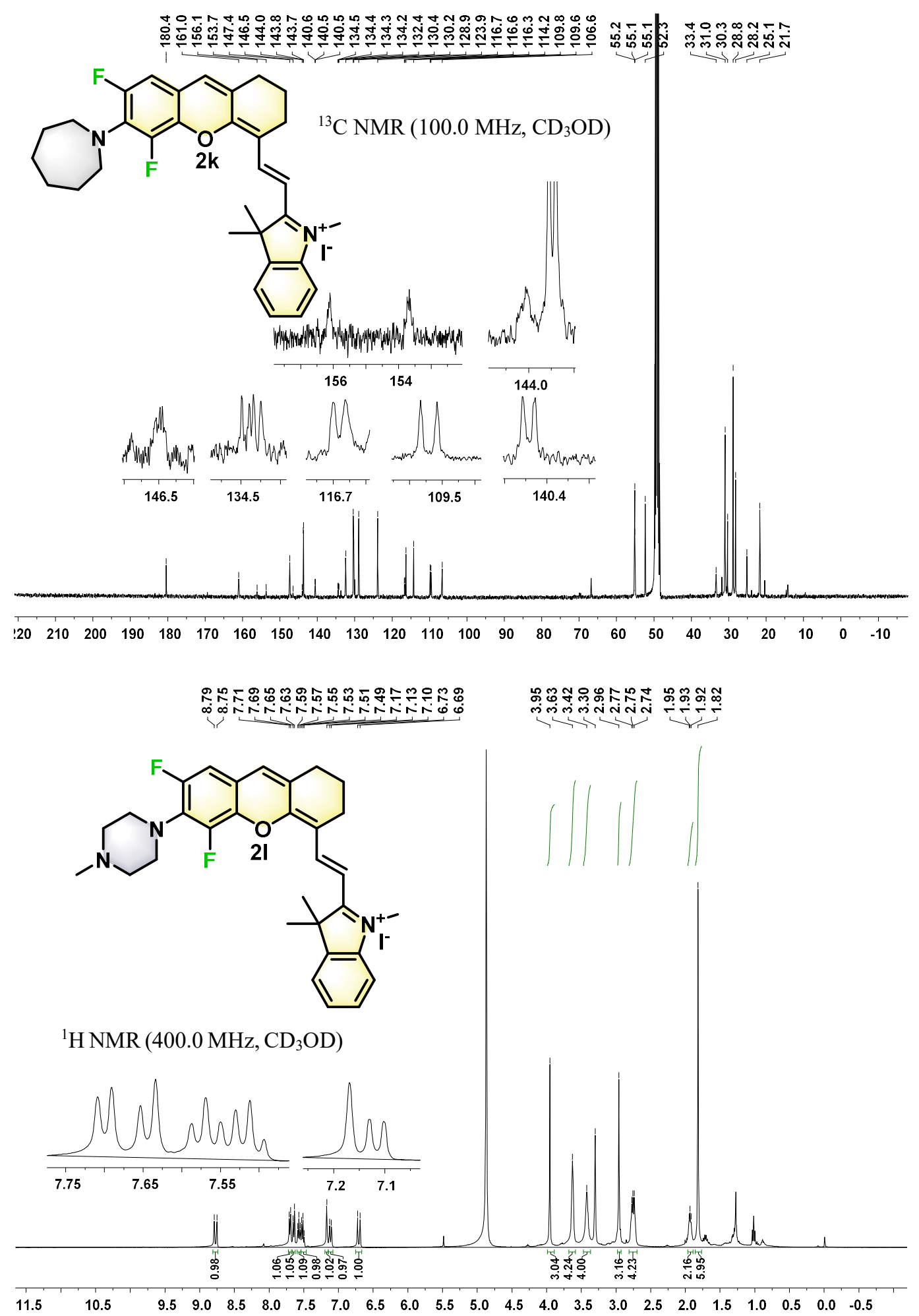


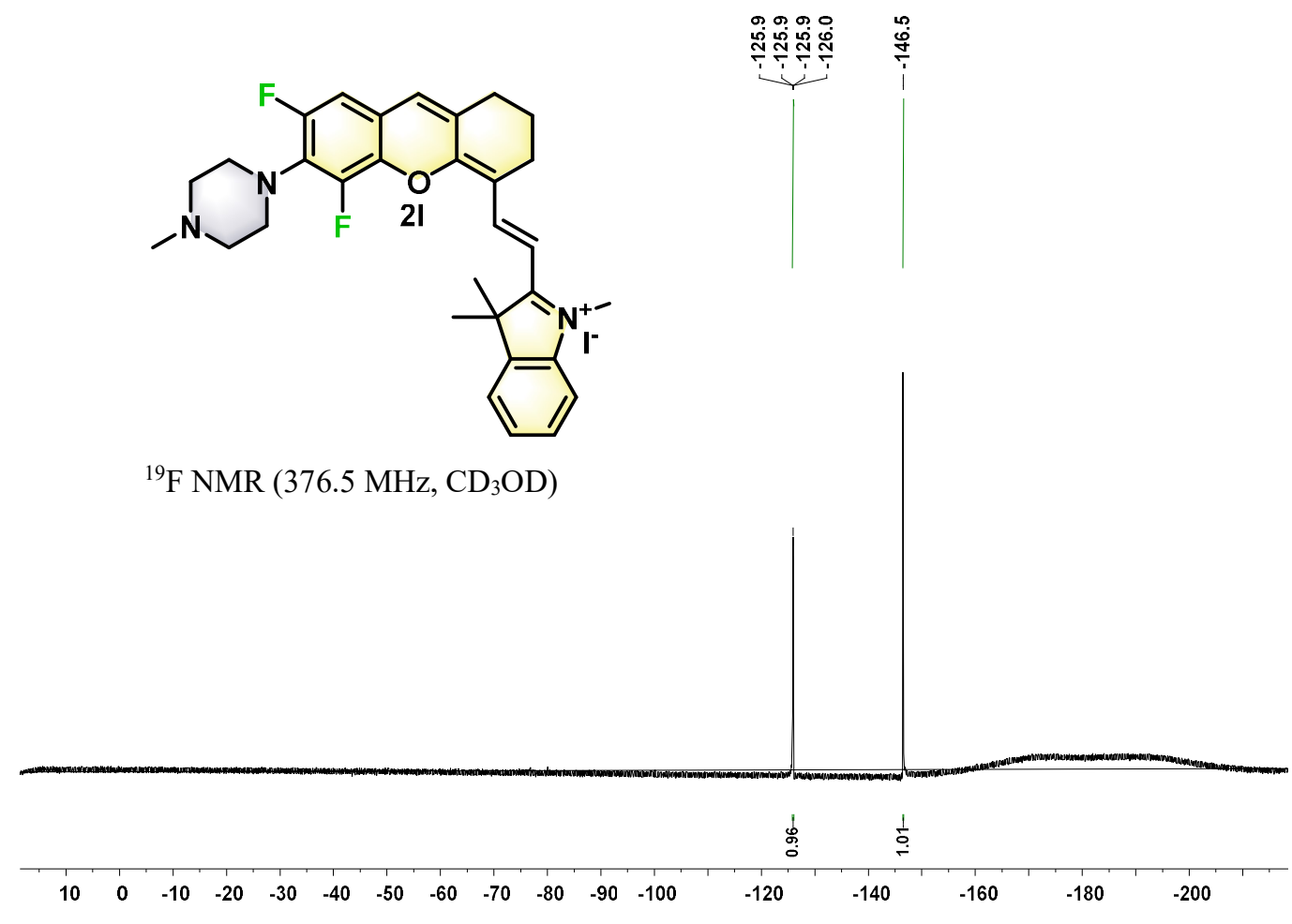

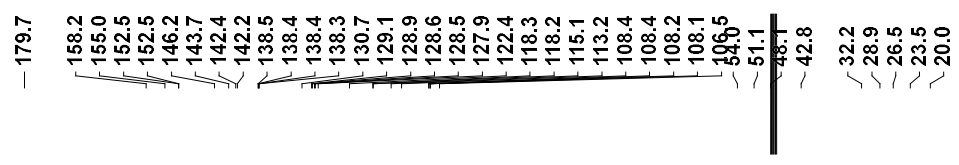

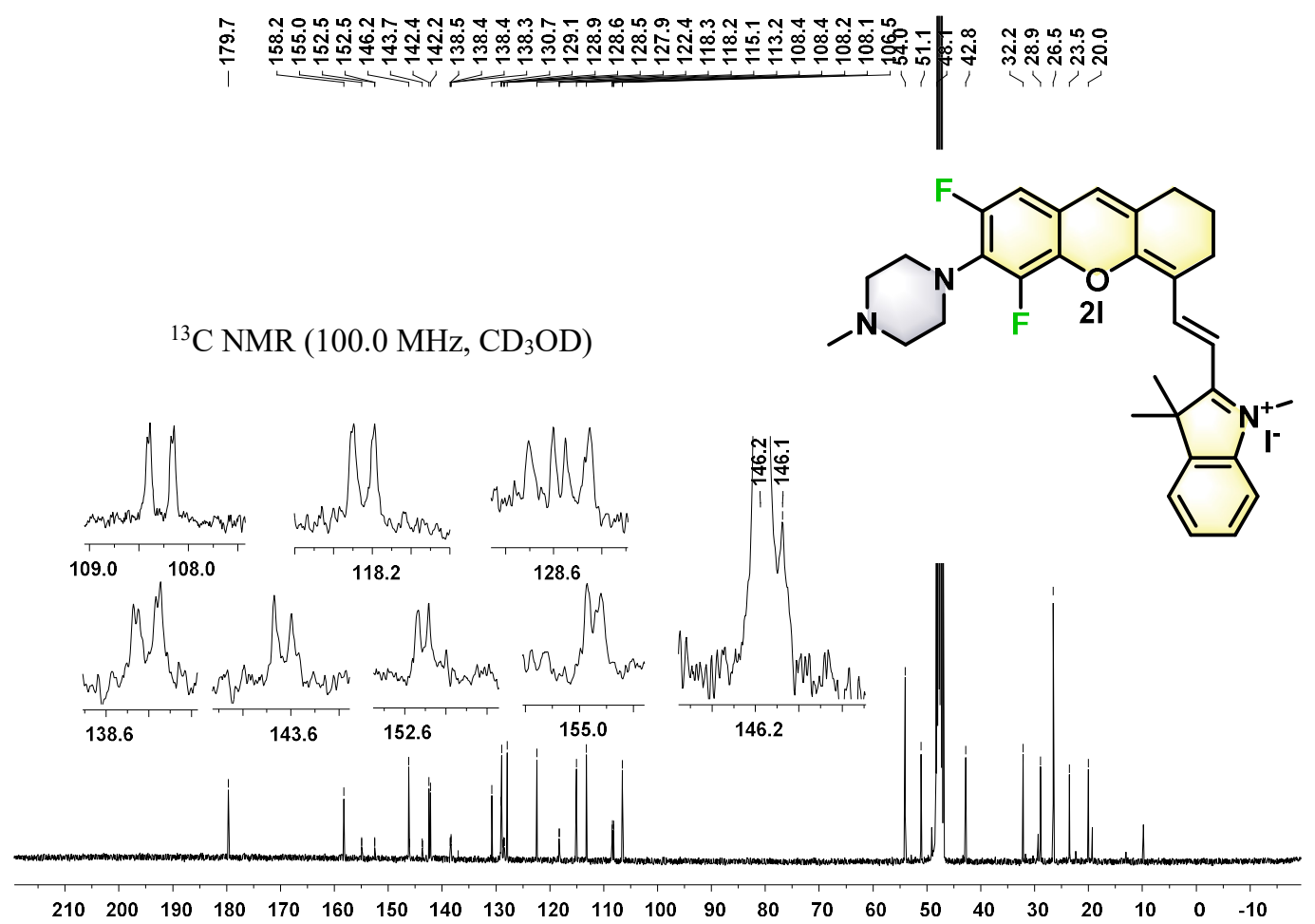



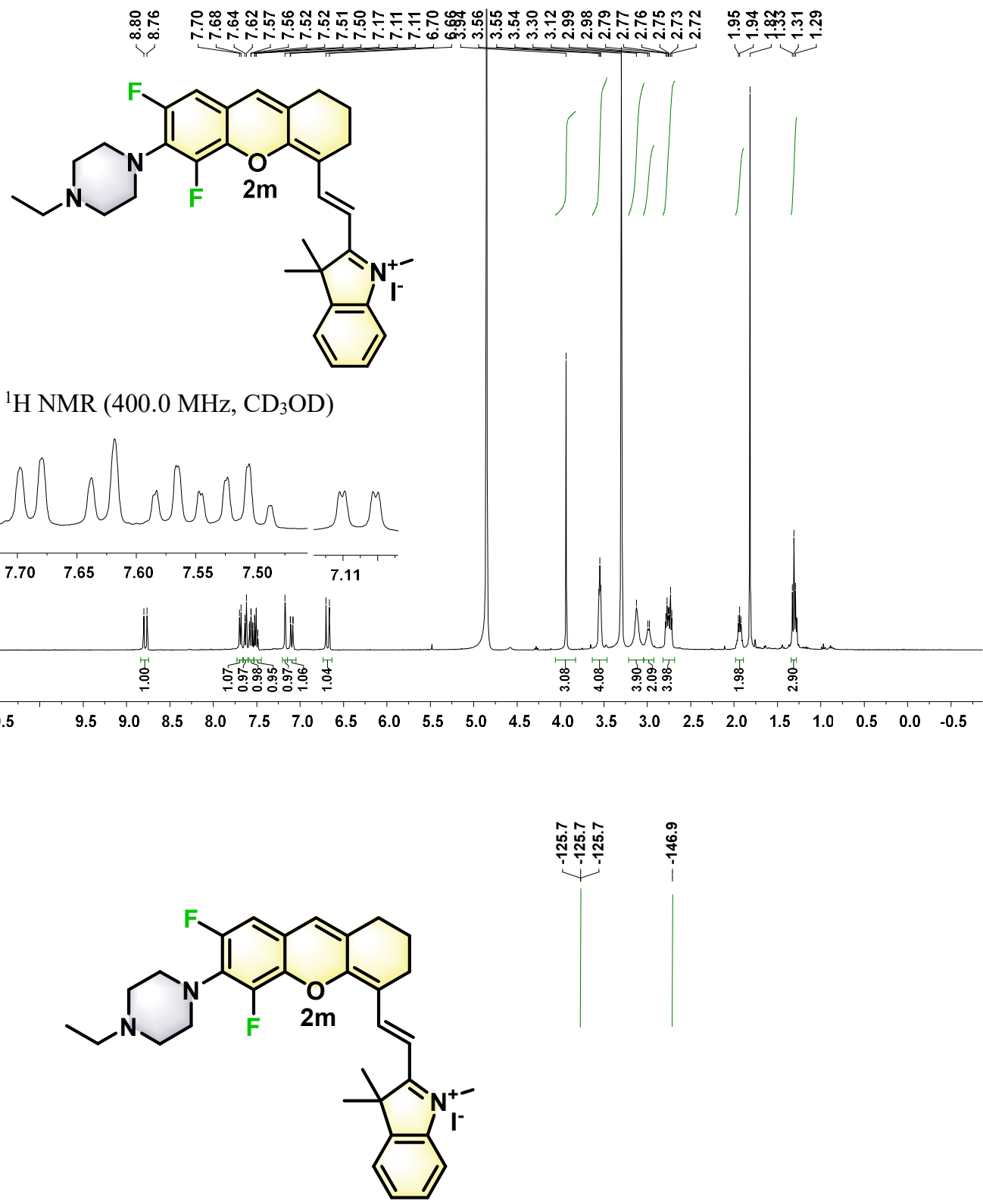

${ }^{19} \mathrm{~F}$ NMR (376.5 MHz, $\left.\mathrm{CD}_{3} \mathrm{OD}\right)$

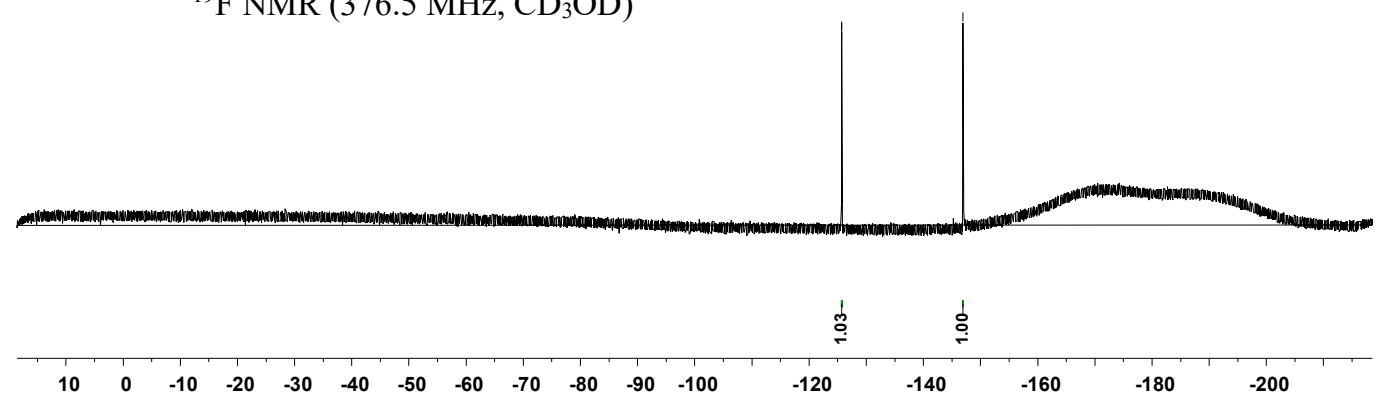



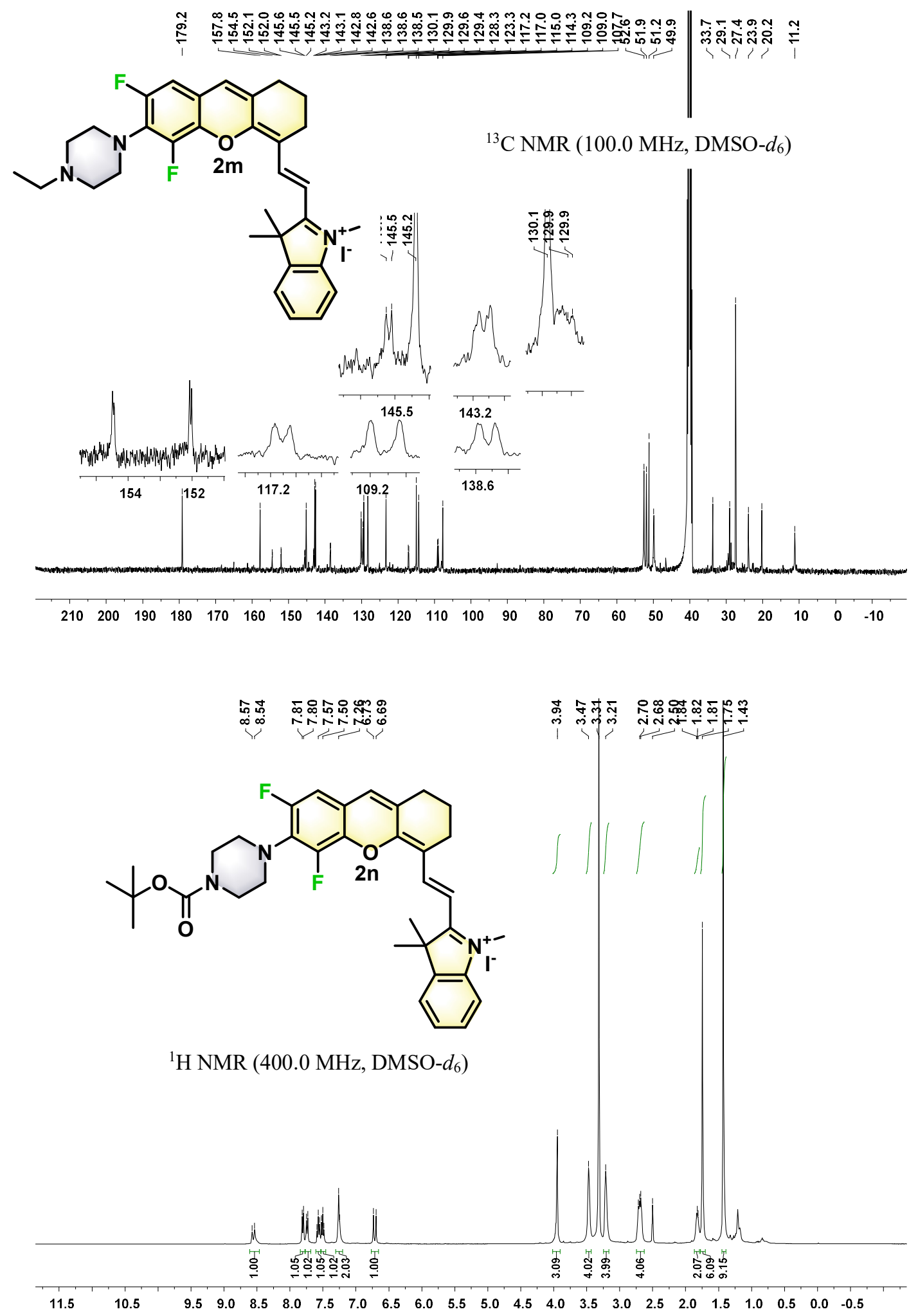


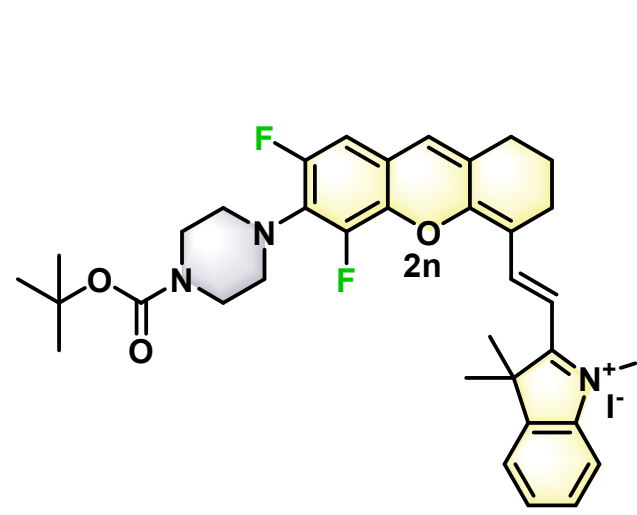

${ }^{19} \mathrm{~F}$ NMR (376.5 MHz, DMSO- $d_{6}$ )
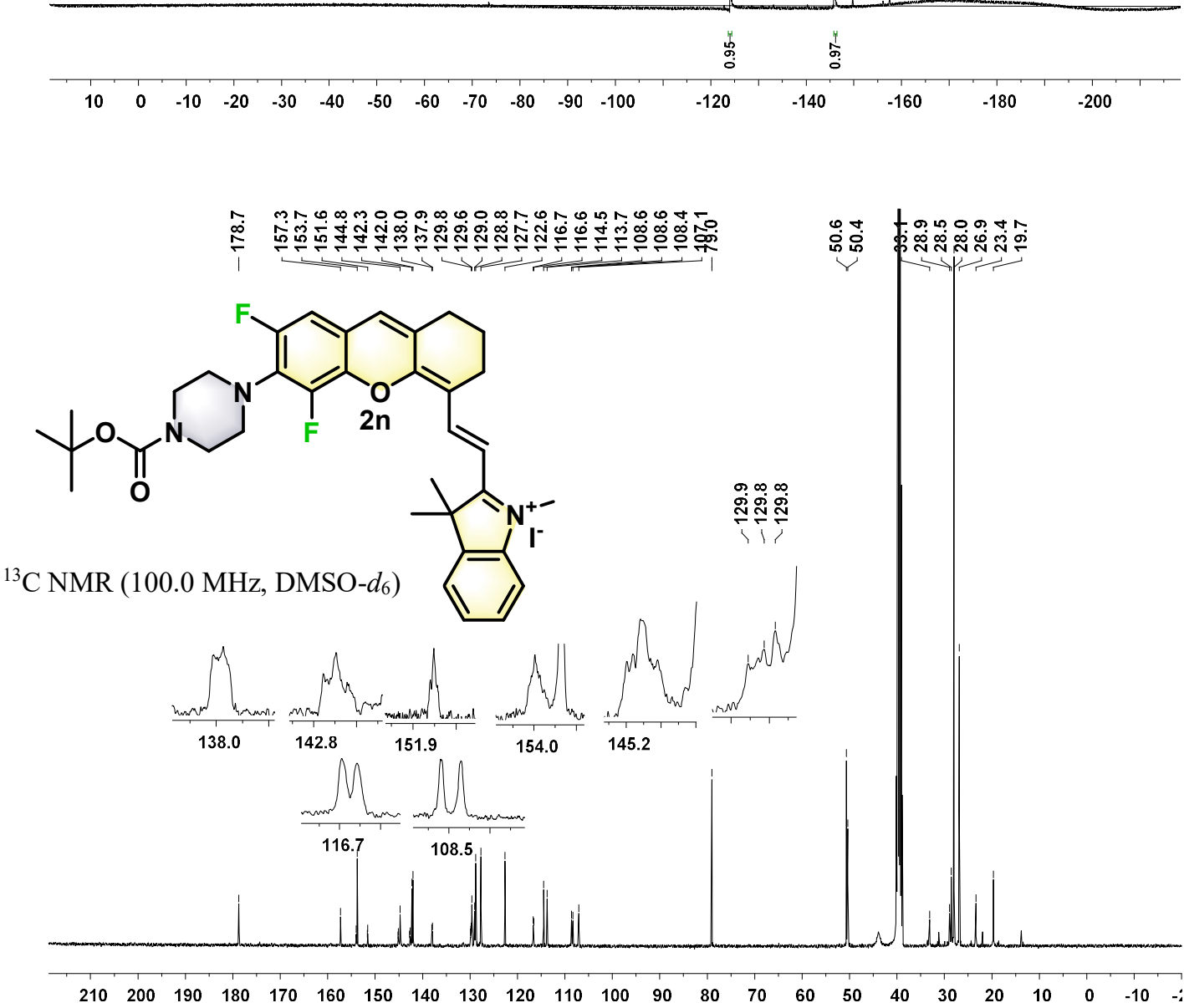


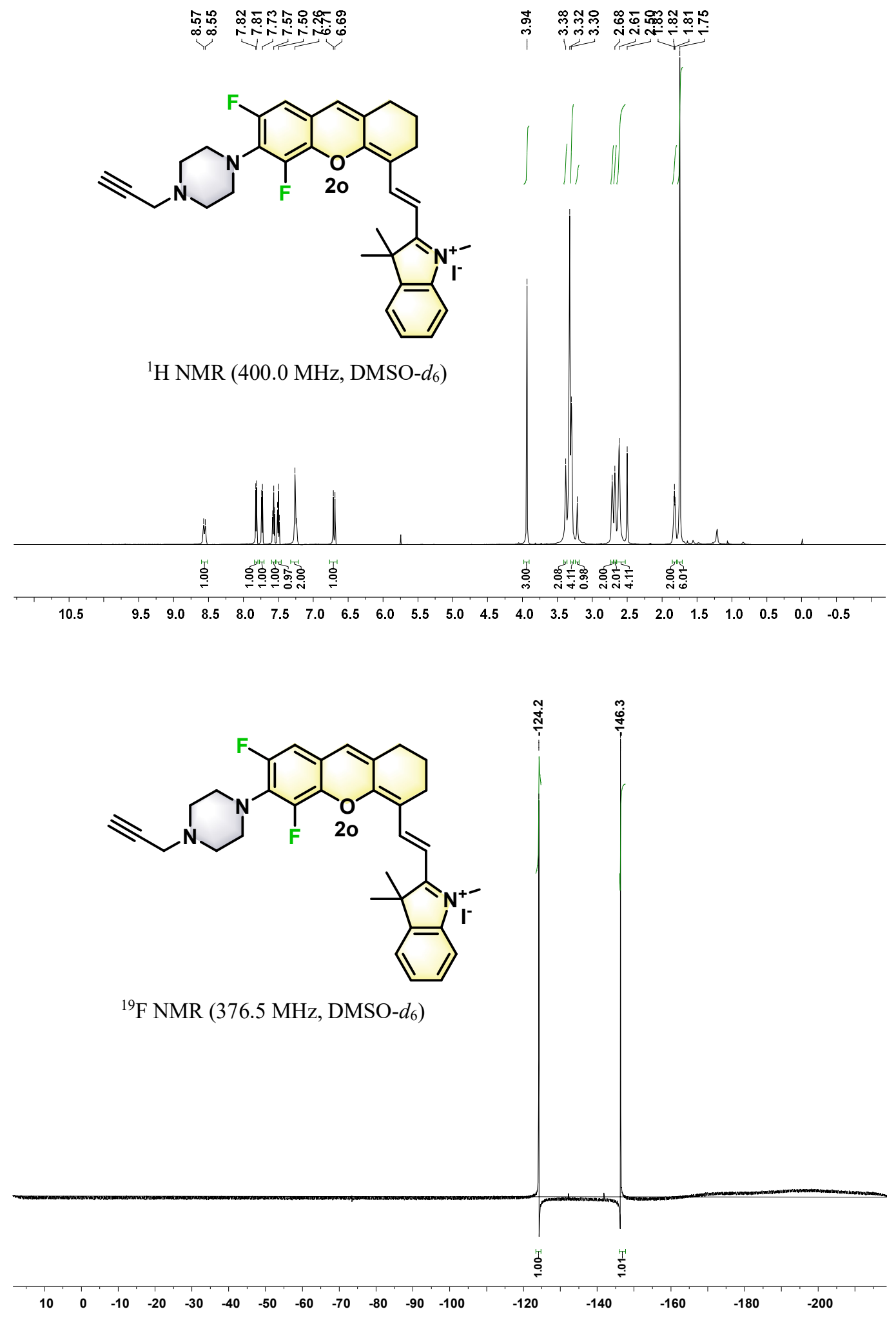



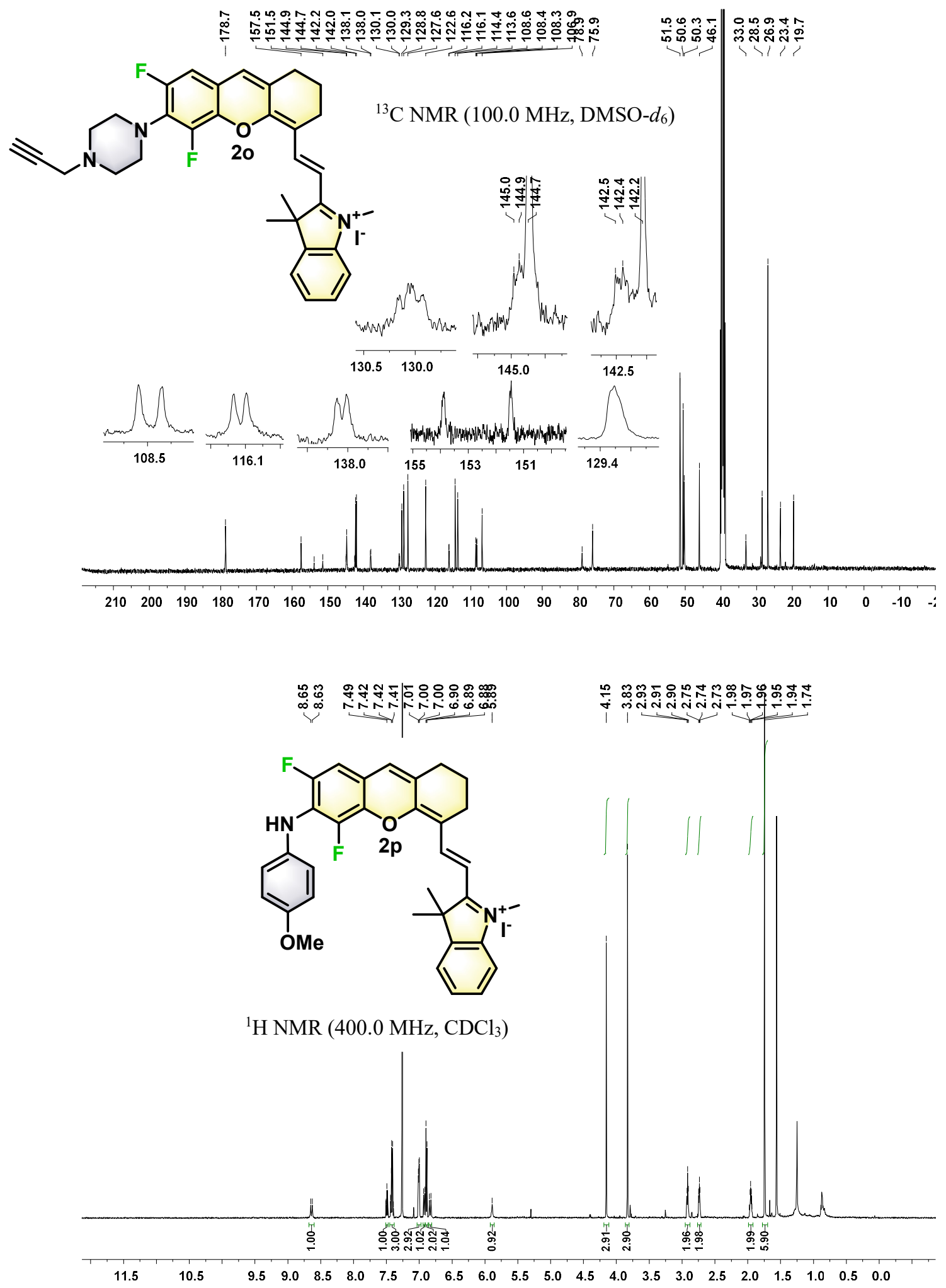


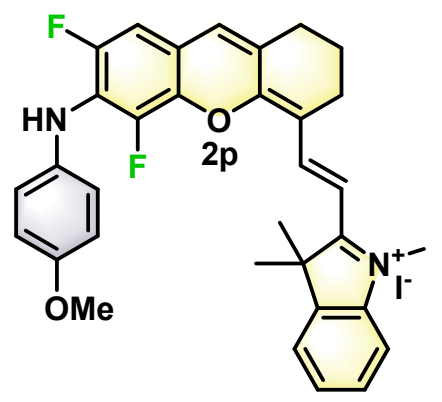

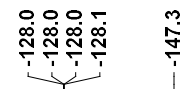

${ }^{19}$ F NMR (376.5 MHz, $\left.\mathrm{CD}_{3} \mathrm{OD}\right)$
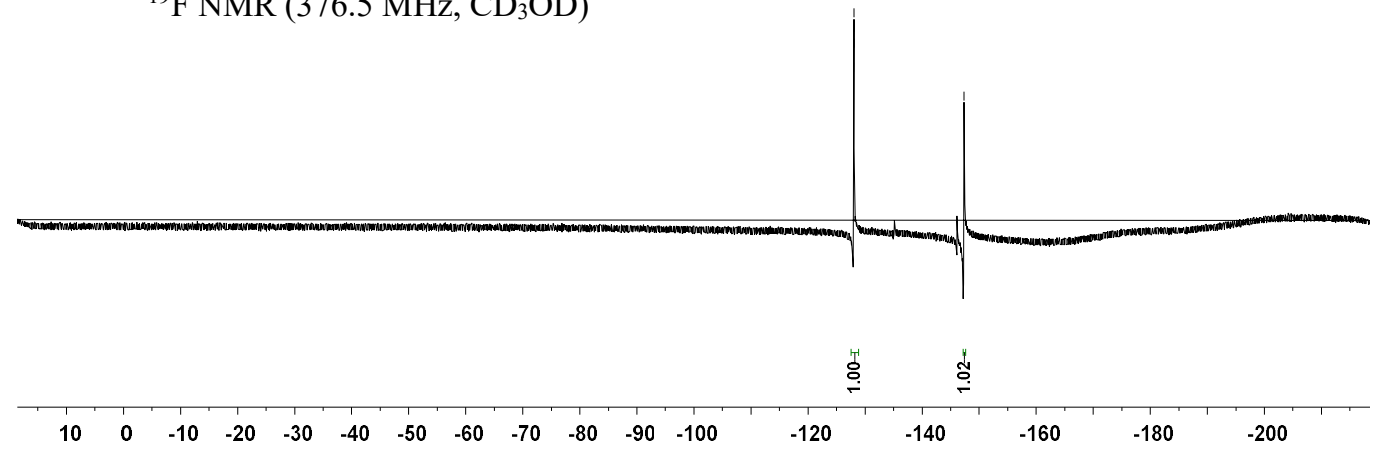

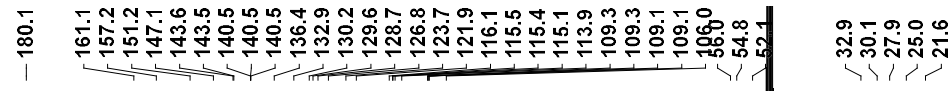
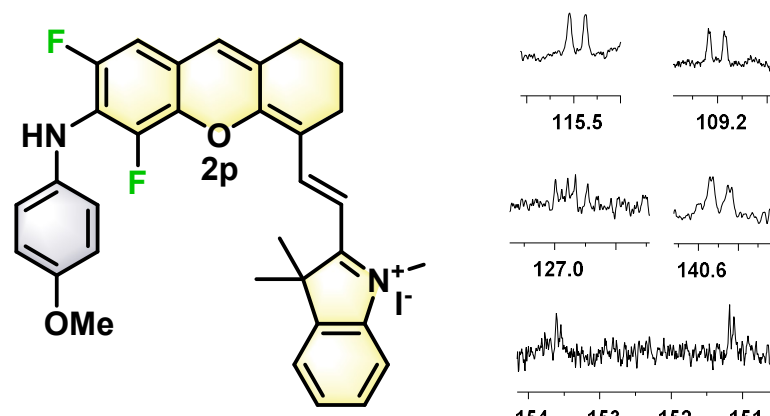

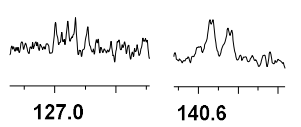

${ }^{13} \mathrm{C}$ NMR (100.0 MHz, $\left.\mathrm{CD}_{3} \mathrm{OD}\right)$
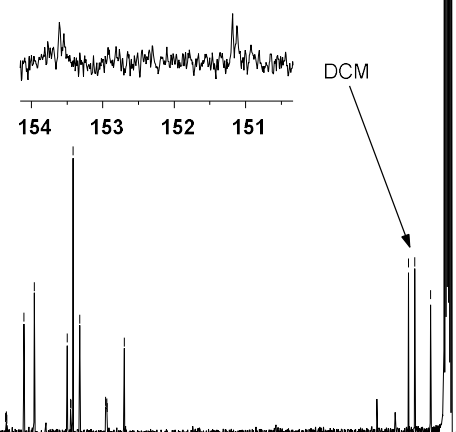

$\begin{array}{llllllllllll}210 & 200 & 190 & 180 & 170 & 160 & 150 & 140 & 130 & 120 & 110 & 100\end{array}$

8070 


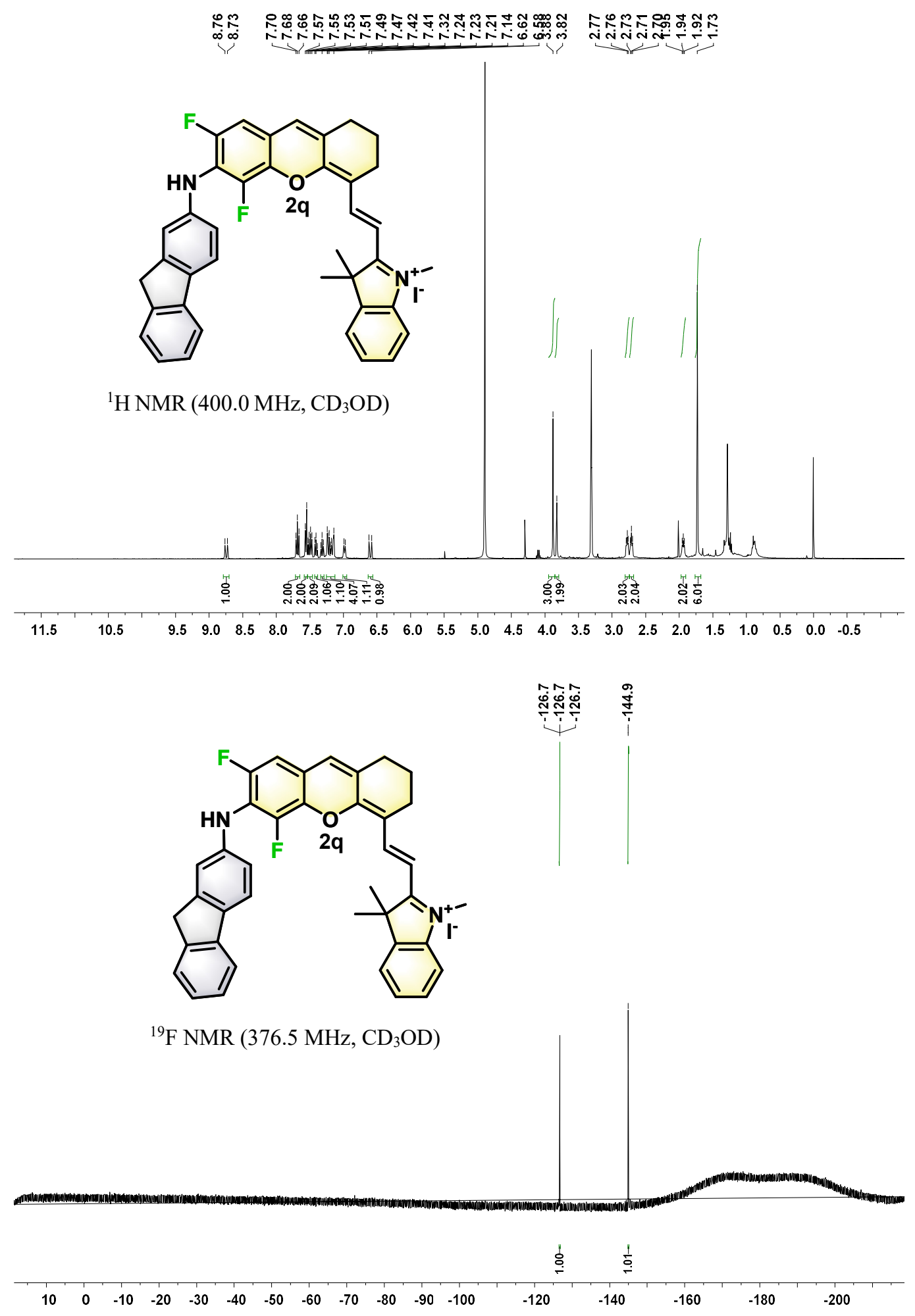



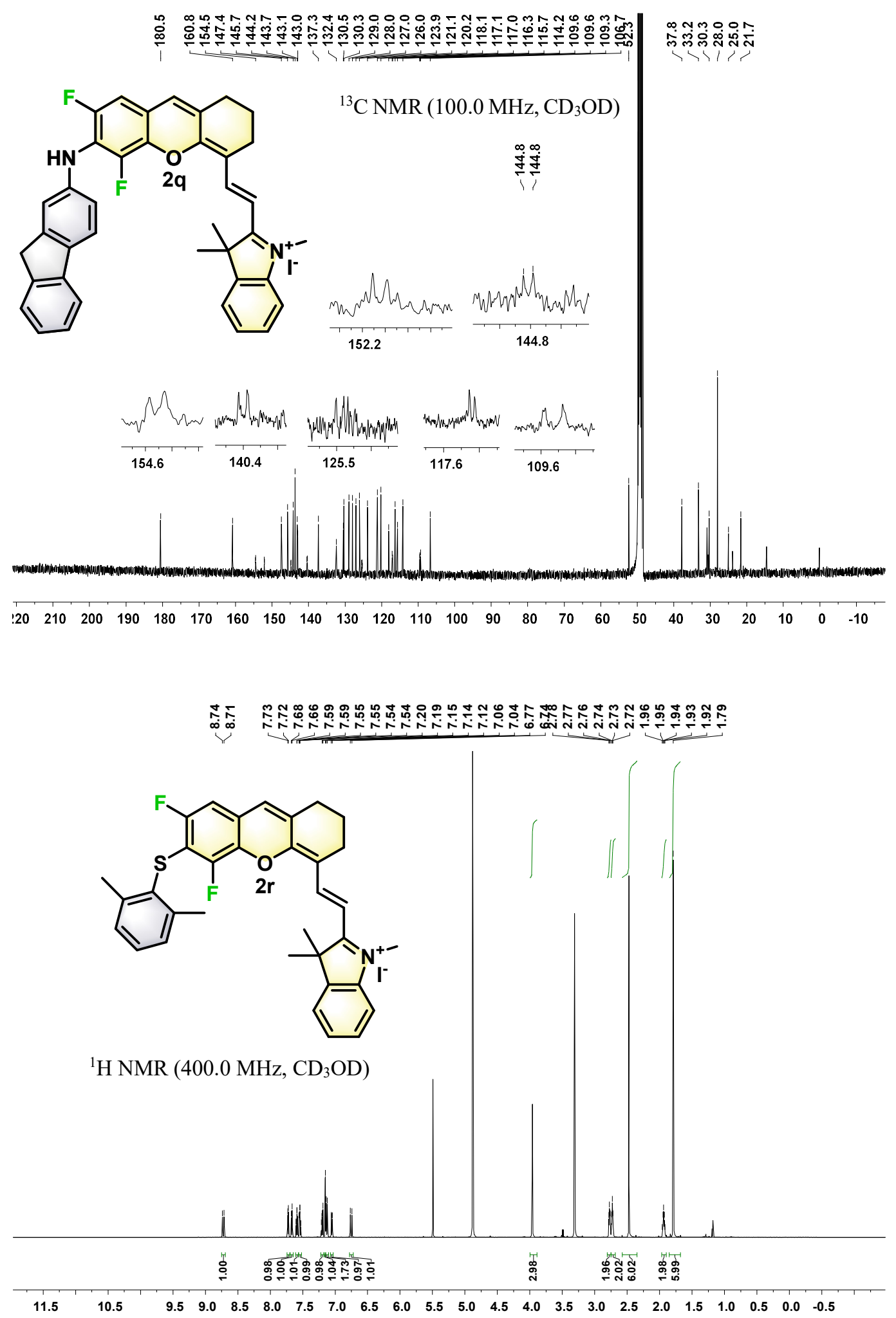


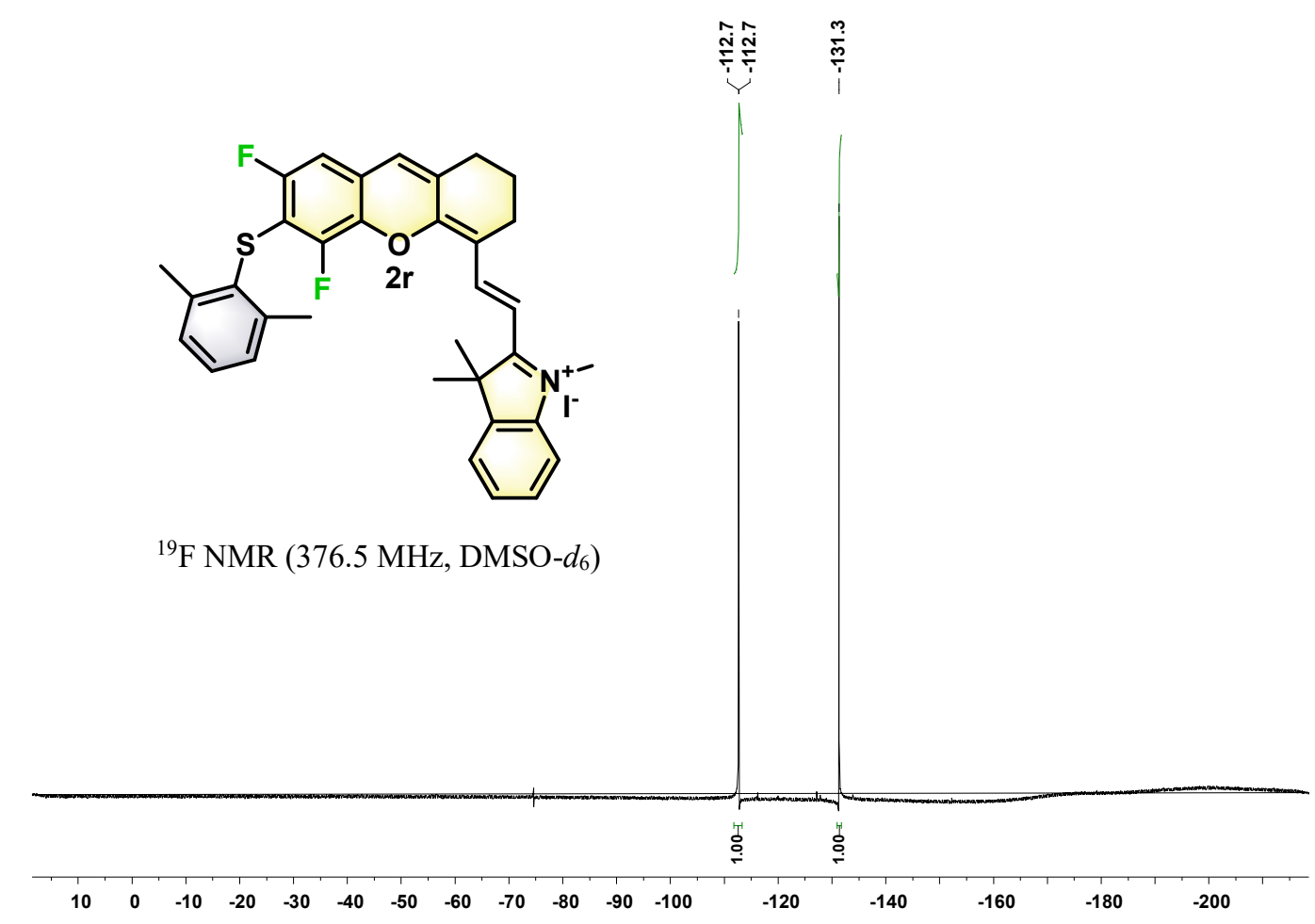

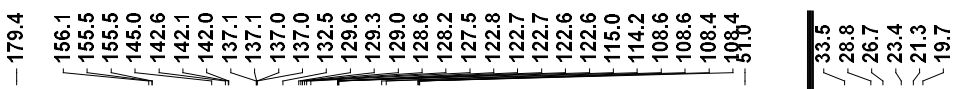

${ }^{13} \mathrm{C}$ NMR $\left(100.0 \mathrm{MHz}\right.$, DMSO- $\left.d_{6}\right)$

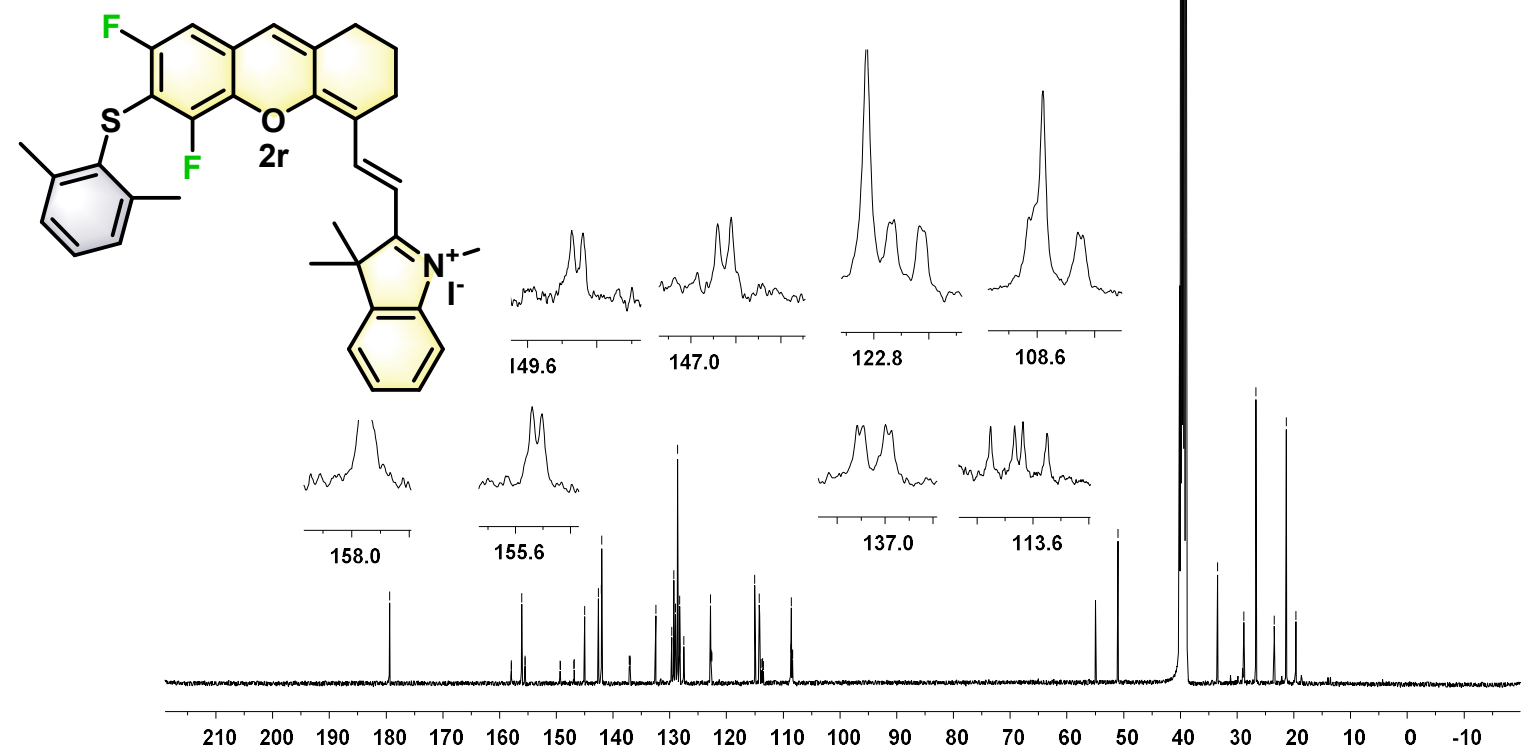




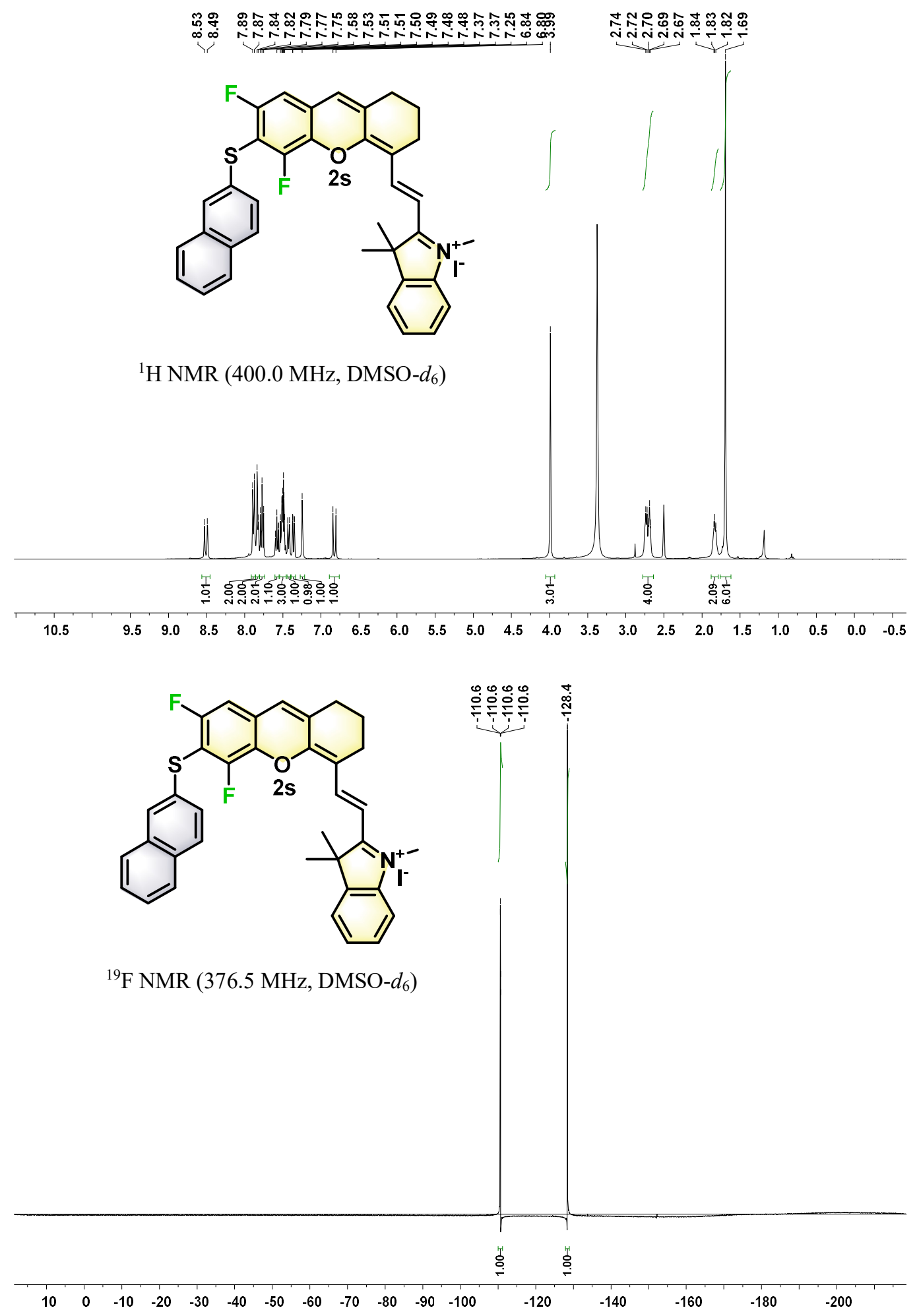




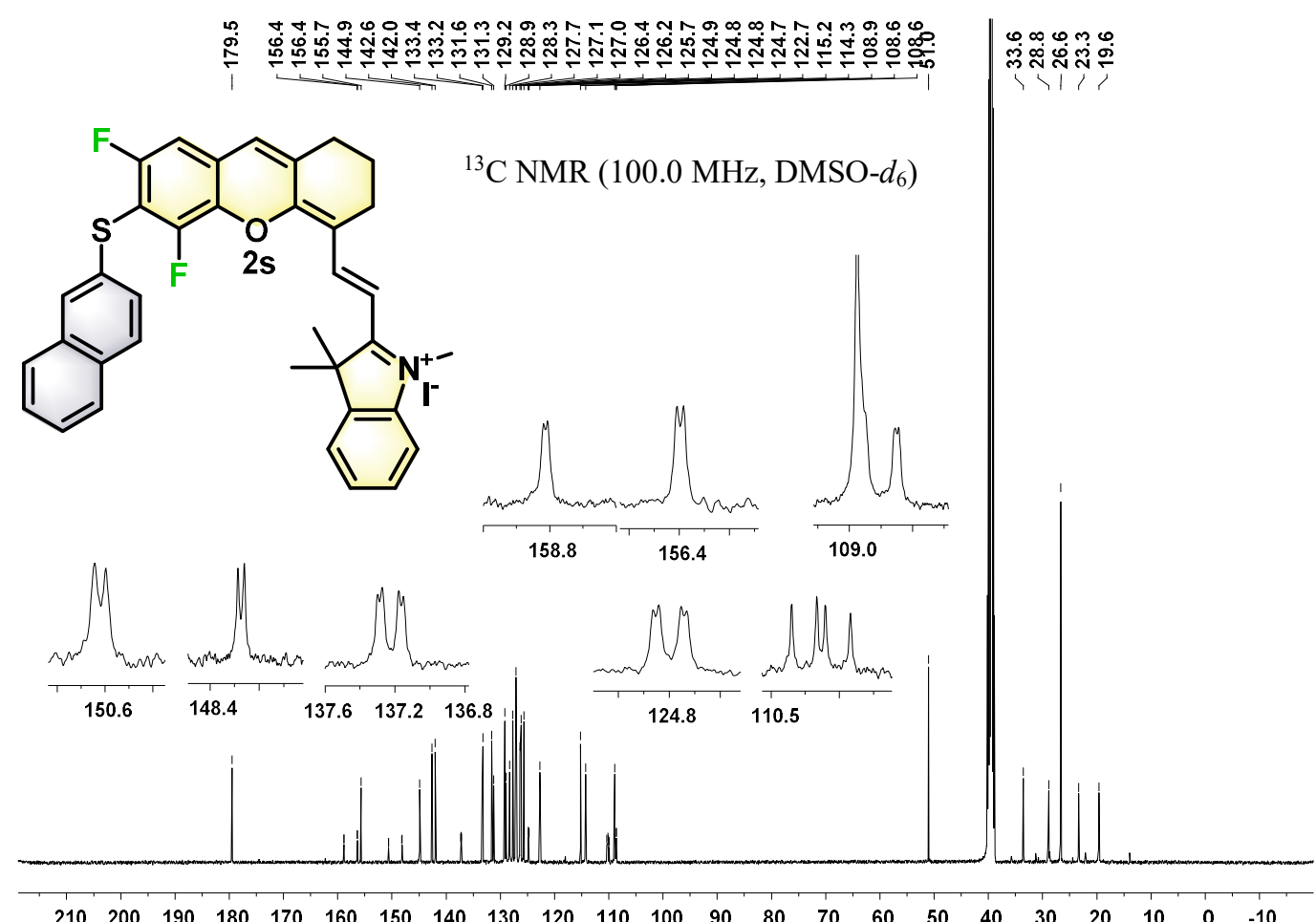

$\begin{array}{lllllllllllllllllllllll}210 & 200 & 190 & 180 & 170 & 160 & 150 & 140 & 130 & 120 & 110 & 100 & 90 & 80 & 70 & 60 & 50 & 40 & 30 & 20 & 10 & 0 & -10\end{array}$
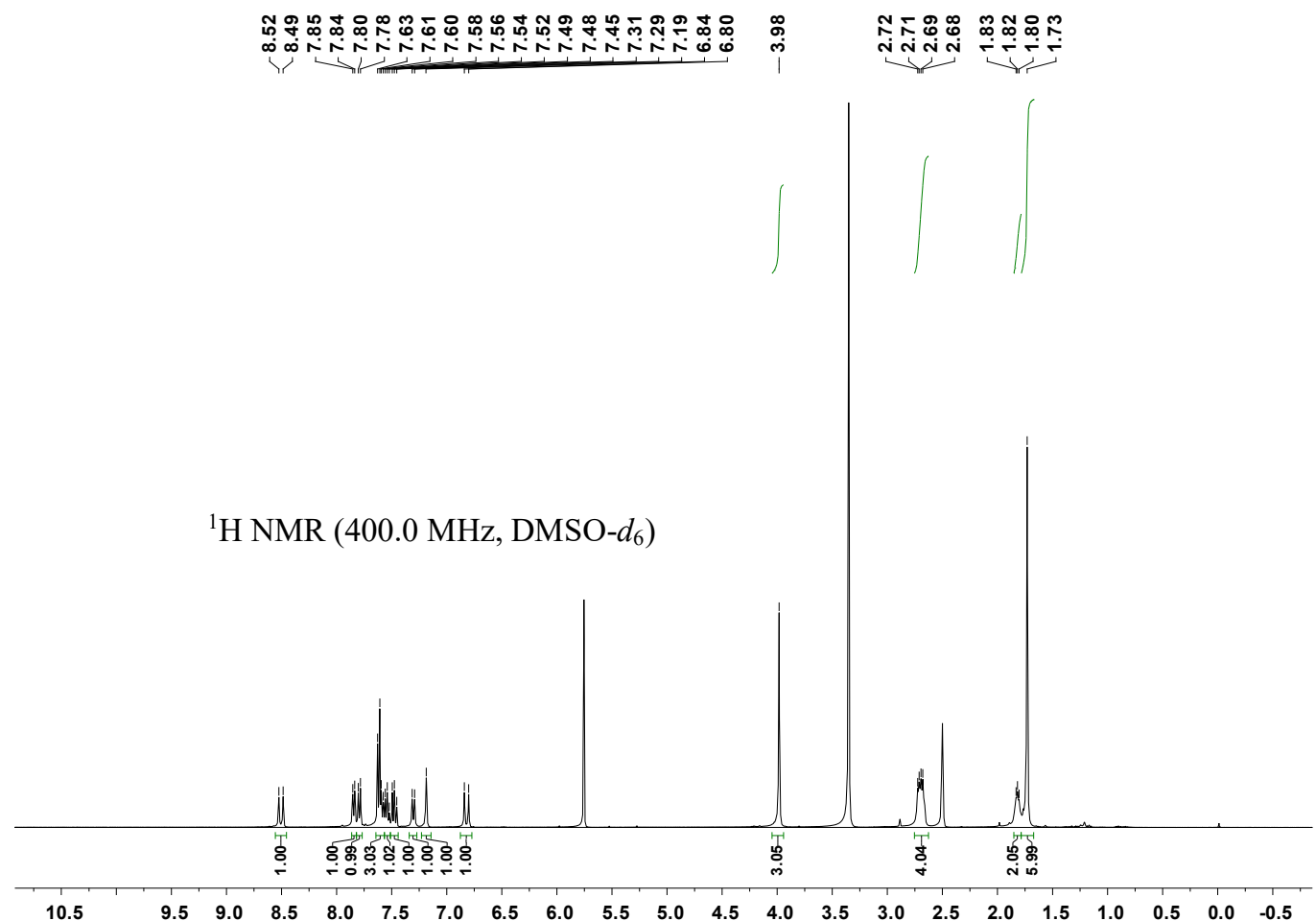


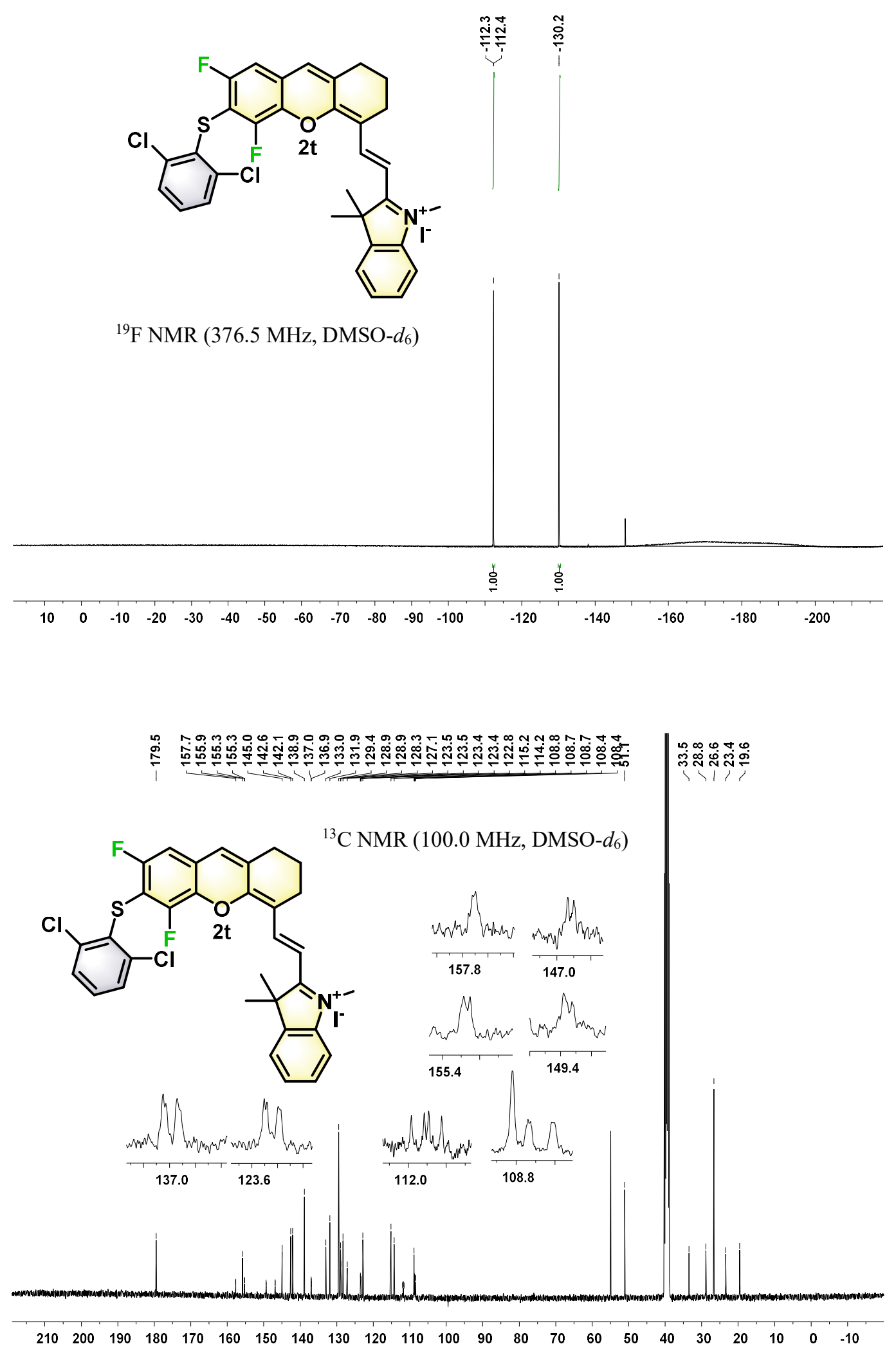



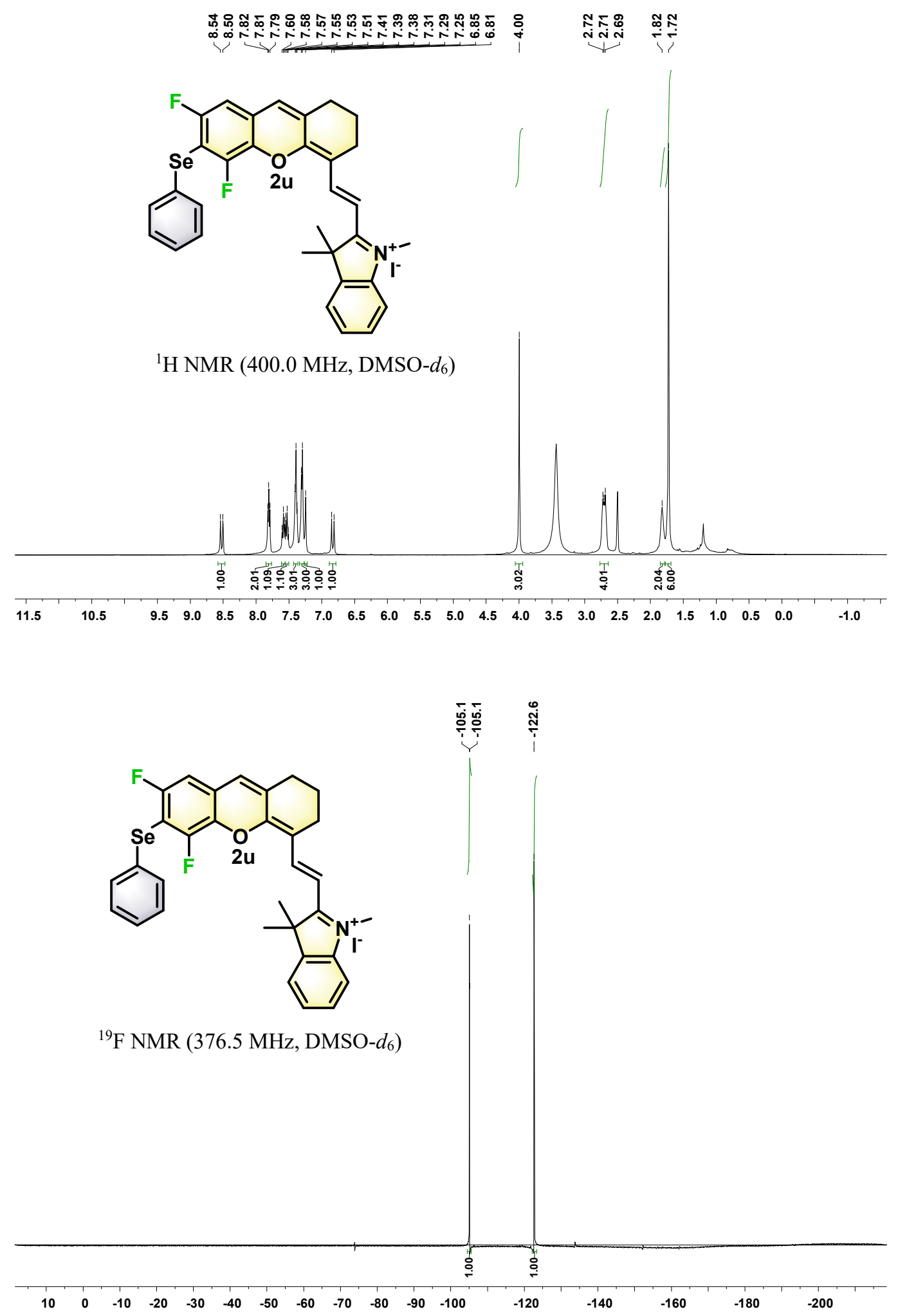

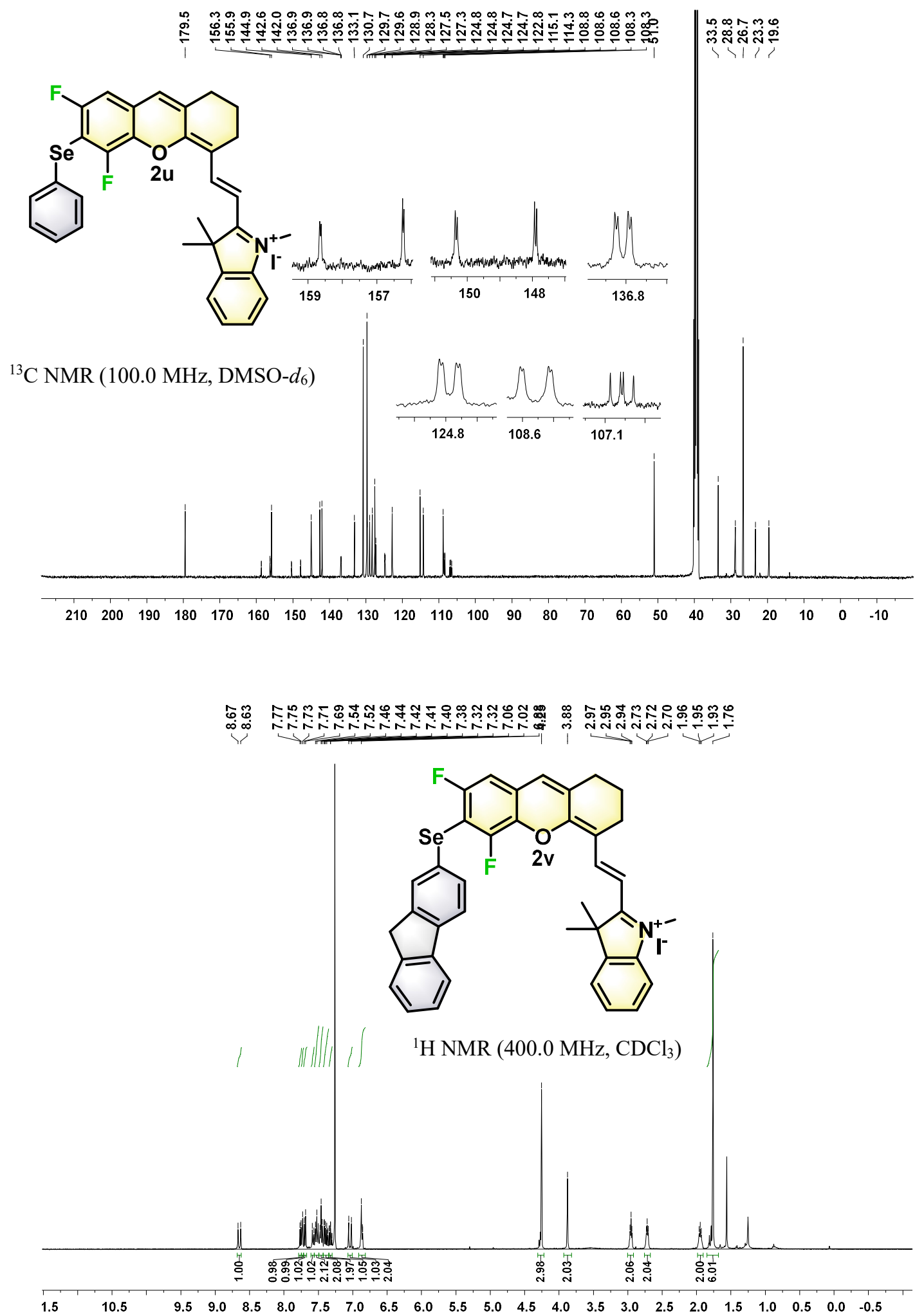


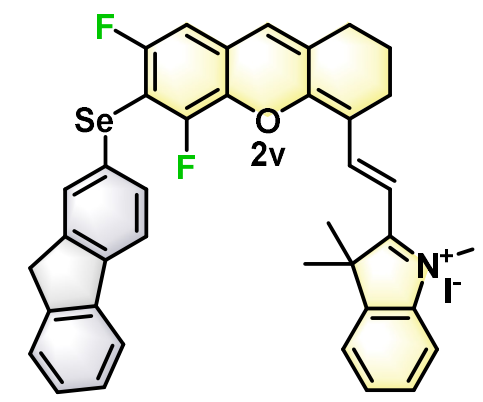

${ }^{19}$ F NMR (376.5 MHz, DMSO- $\left.d_{6}\right)$

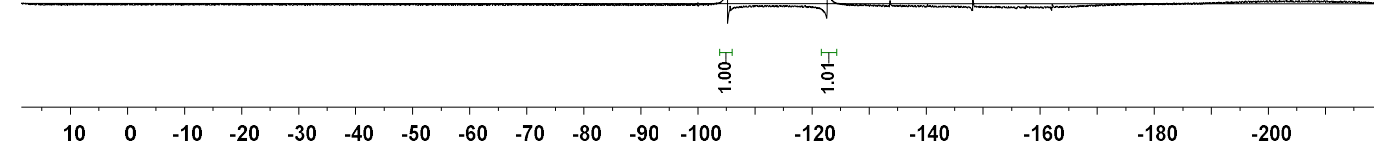

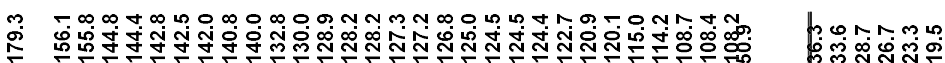

1 年

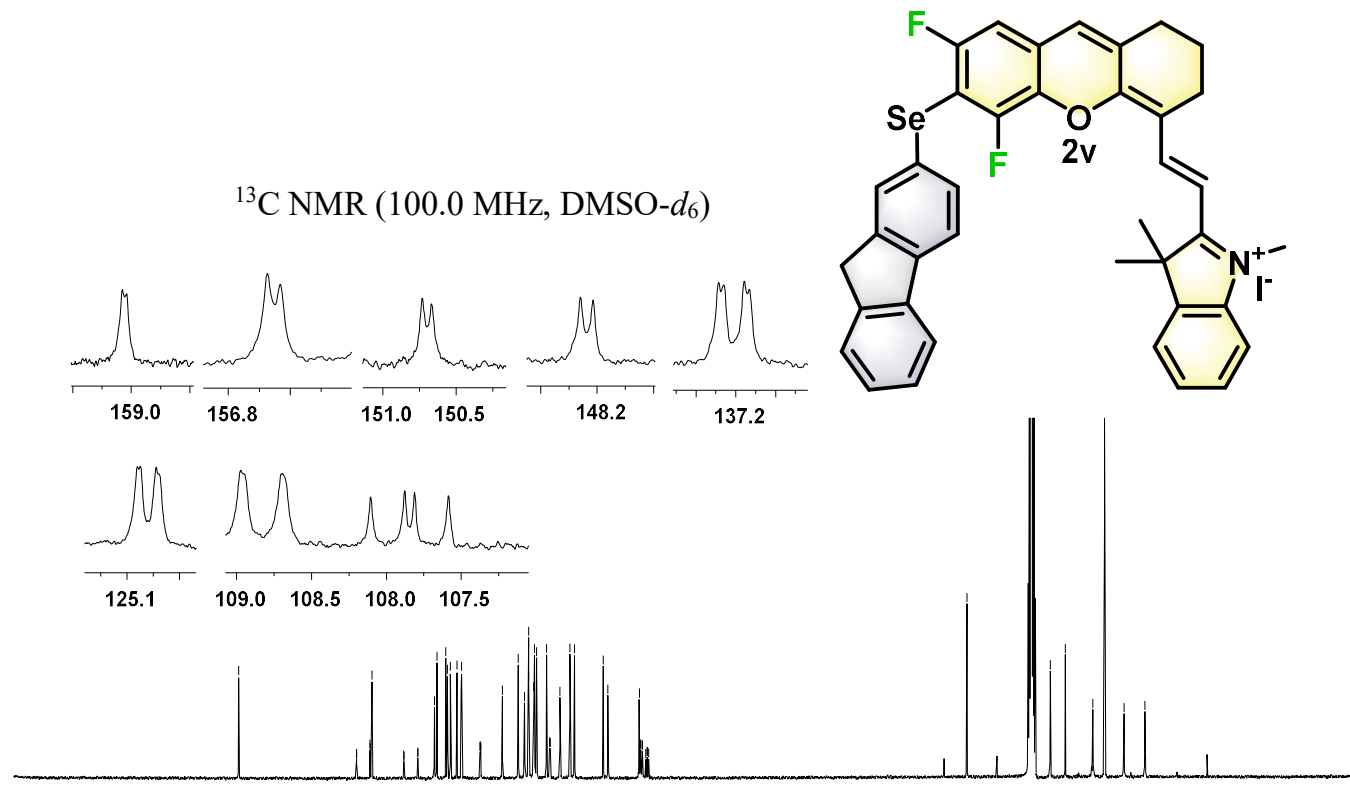

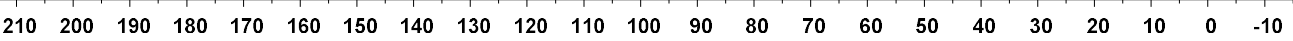




\section{High-performance liquid chromatography (HPLC) separations}

The following chromatographic system was used for the analytical experiments: System A: RP-HPLC (Phenomenex Kinetex $\mathrm{C}_{18}$ column, $\left.2.6 \mu \mathrm{m}, 2.1 \times 50 \mathrm{~mm}\right)$ with $\mathrm{CH}_{3} \mathrm{CN}(+0.1 \% \mathrm{FA})$ and $0.1 \%$ aq. formic acid (aq. FA, $\left.\mathrm{pH} 2.5\right)$ as eluents $\left[5 \% \mathrm{CH}_{3} \mathrm{CN}(0.1 \mathrm{~min})\right.$ followed by linear gradient from $5 \%$ to $100 \% \mathrm{CH}_{3} \mathrm{CN}(5 \mathrm{~min})$, then $\left.100 \% \mathrm{CH}_{3} \mathrm{CN}(4 \mathrm{~min})\right]$ at a flow rate of $0.5 \mathrm{~mL} / \mathrm{min}$. UV-visible detection was achieved at 220, 260, 600 and $700 \mathrm{~nm}$ (+ diode array detection in the range 220-800 nm). Low resolution ESI-MS detection in the positive mode (full scan, 100-1500 a.m.u., data type: centroid, needle voltage: $3.0 \mathrm{kV}$, probe temperature: $350^{\circ} \mathrm{C}$, cone voltage: $75 \mathrm{~V}$ and scan time: $1 \mathrm{~s}$ ).

RP-HPLC elution profile of 1 (system A, detection at $600 \mathrm{~nm}$ )

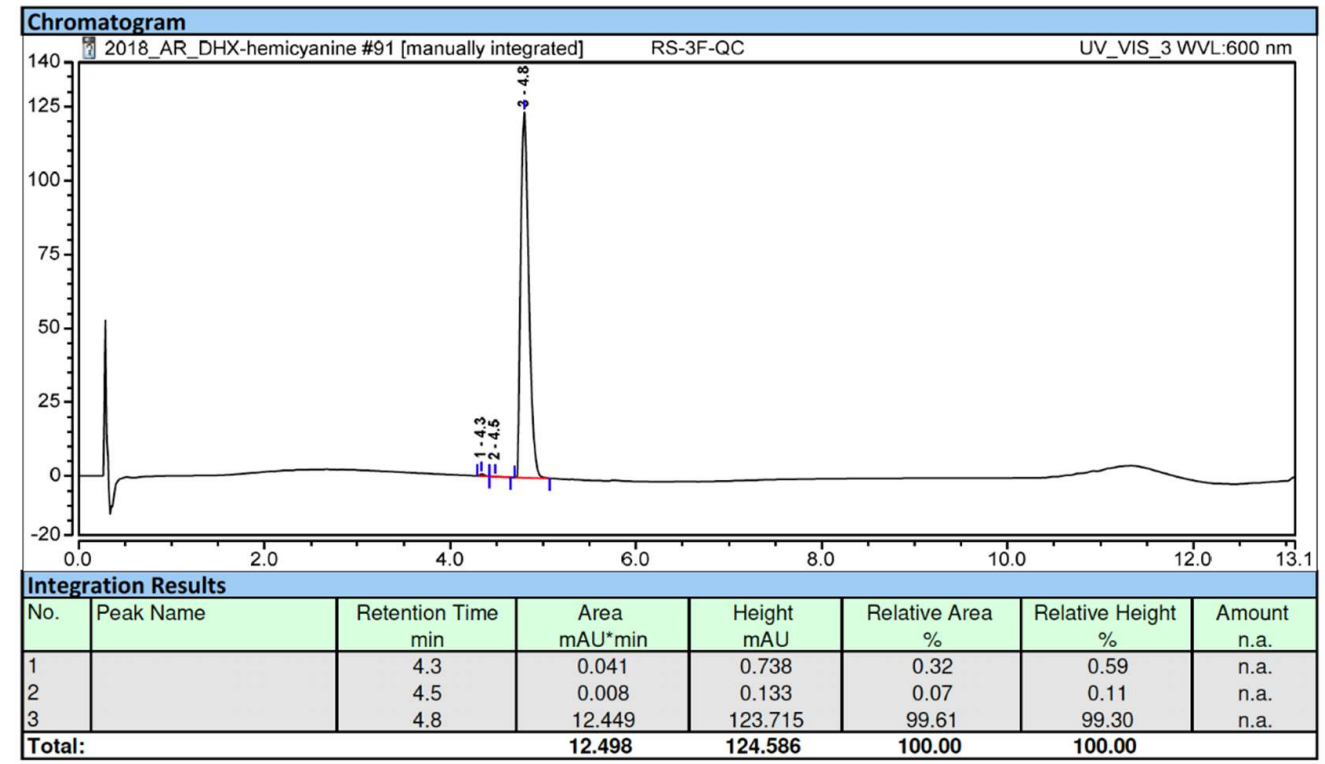


RP-HPLC elution profile of $\mathbf{2 l}$ (system A, detection at $600 \mathrm{~nm}$ )

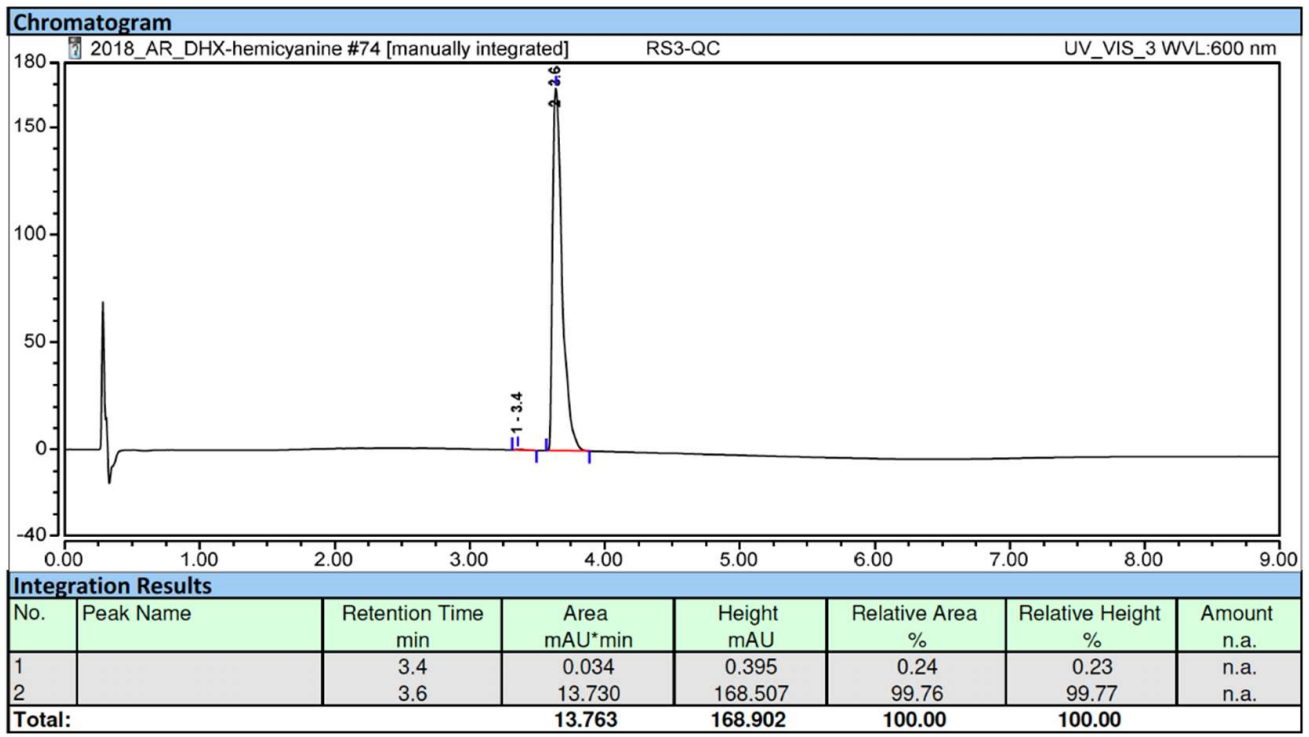

RP-HPLC elution profile of $\mathbf{2 m}$ (system A, detection at $600 \mathrm{~nm}$ )

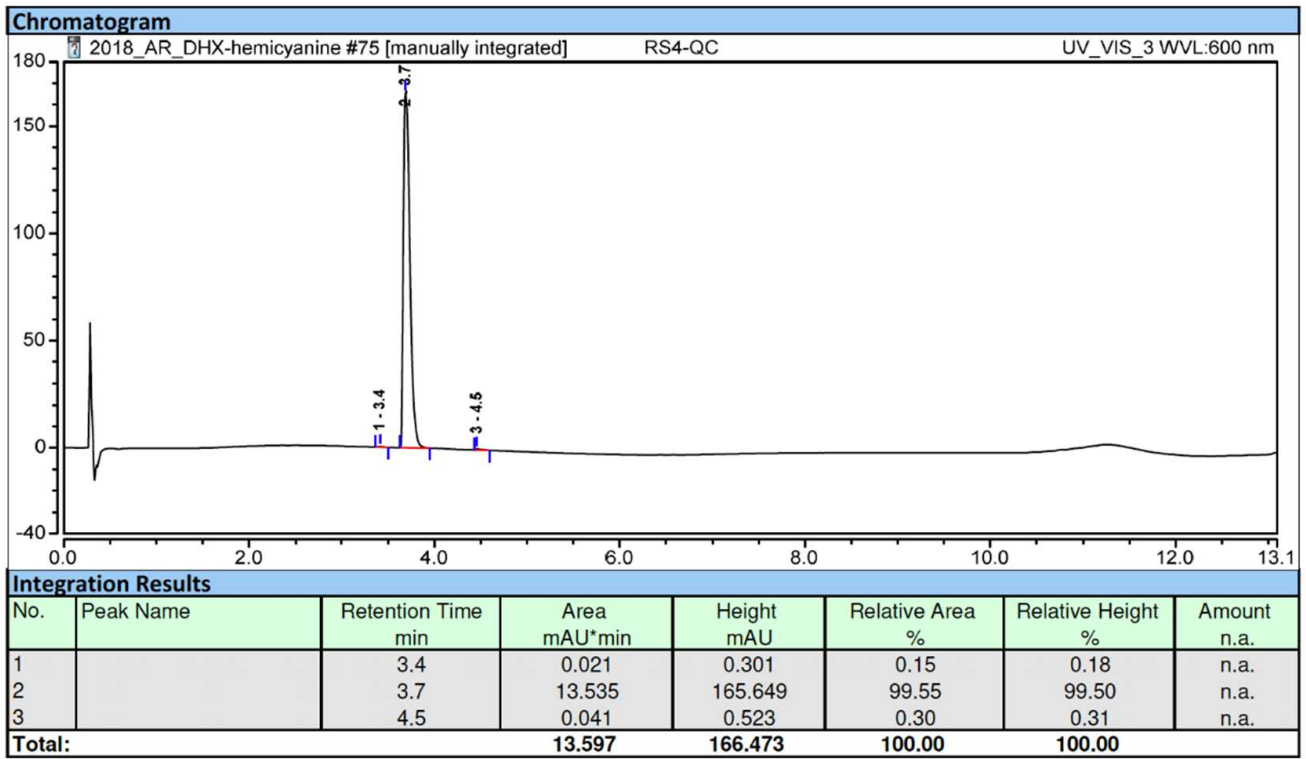


RP-HPLC elution profile of $\boldsymbol{8}$ (system A, detection at $600 \mathrm{~nm}$ )

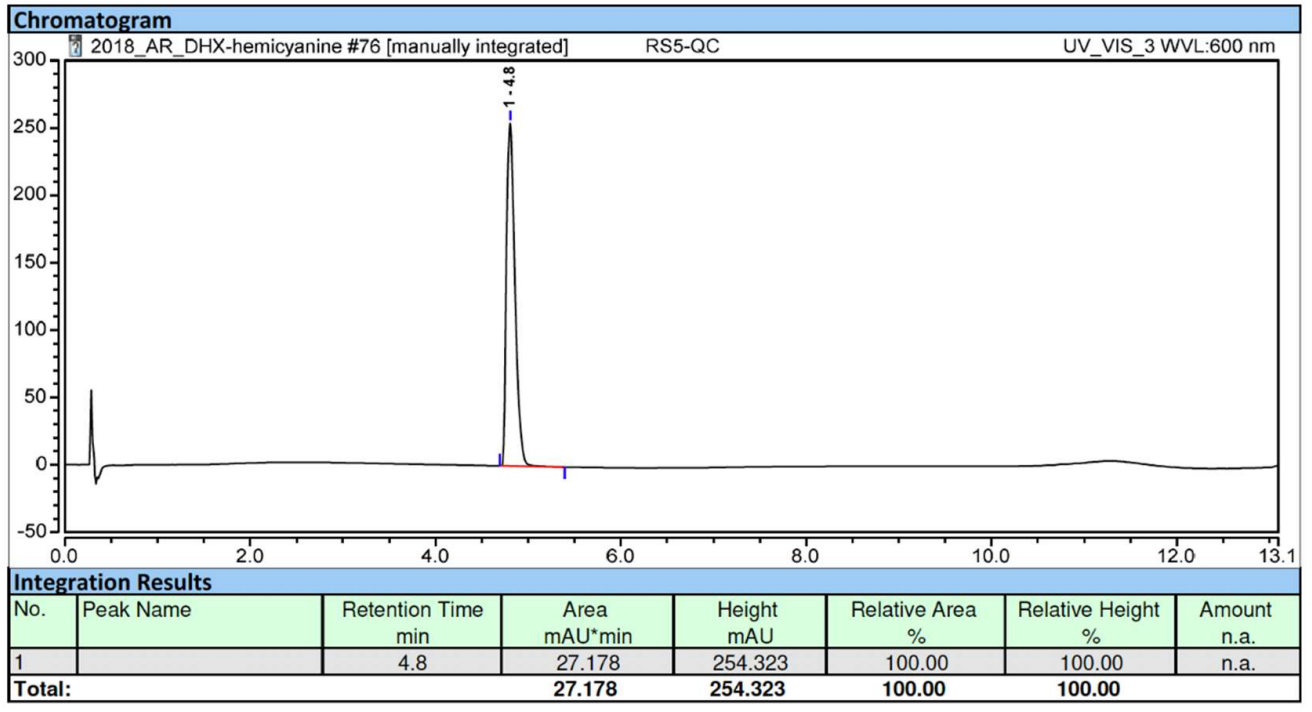

RP-HPLC elution profile of $\mathbf{2 i}($ system A, detection at $600 \mathrm{~nm})$

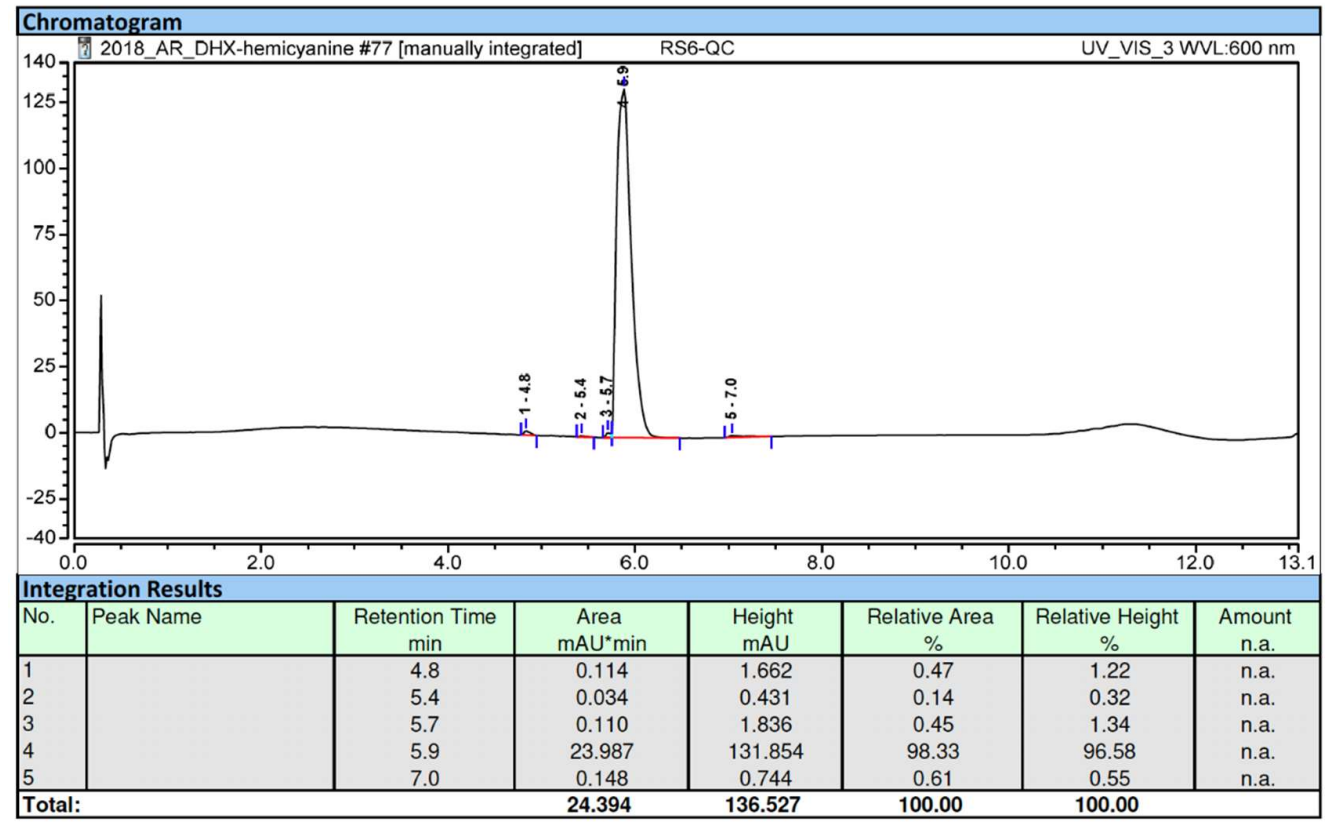




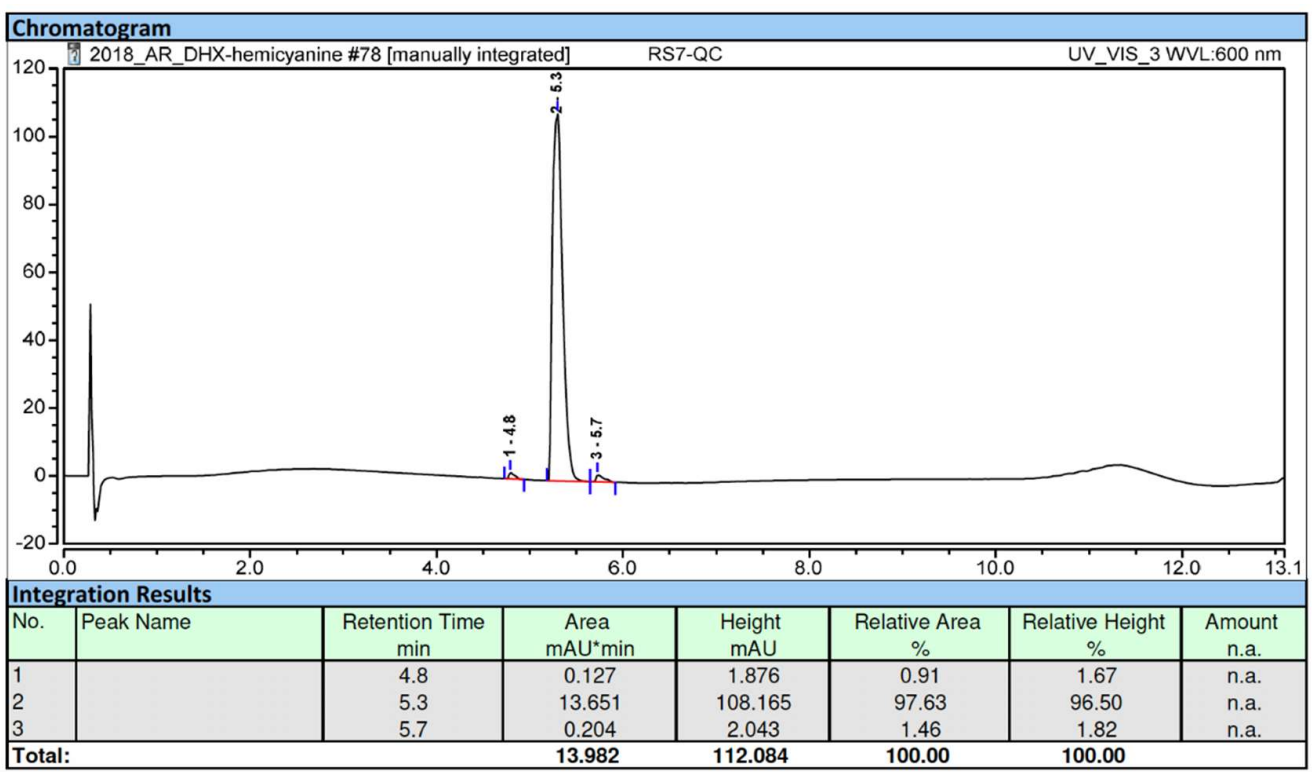

$R P-H P L C$ elution profile of $\mathbf{2 n}$ (system A, detection at $600 \mathrm{~nm}$ )

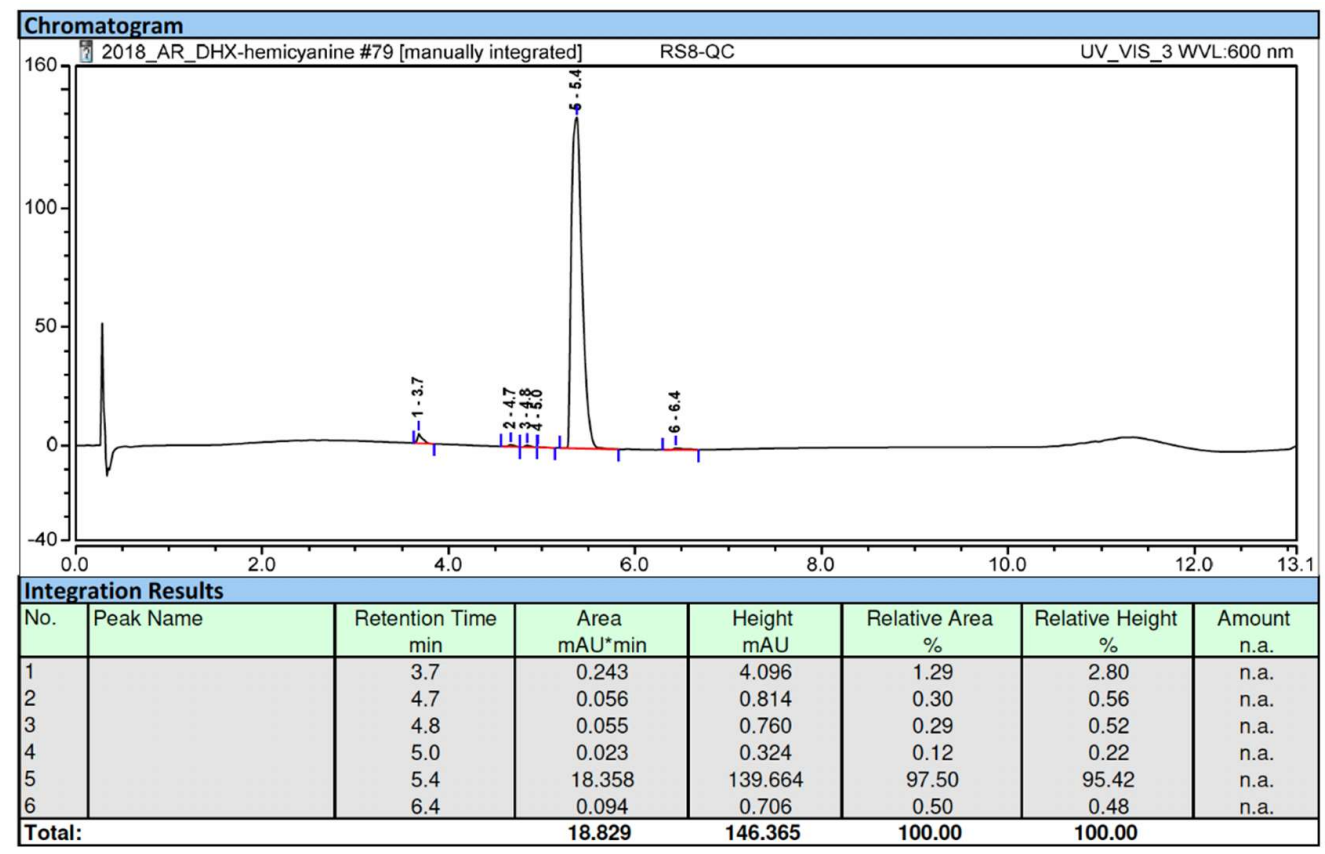


RP-HPLC elution profile of $\mathbf{2 o}$ (system A, detection at $600 \mathrm{~nm}$ )

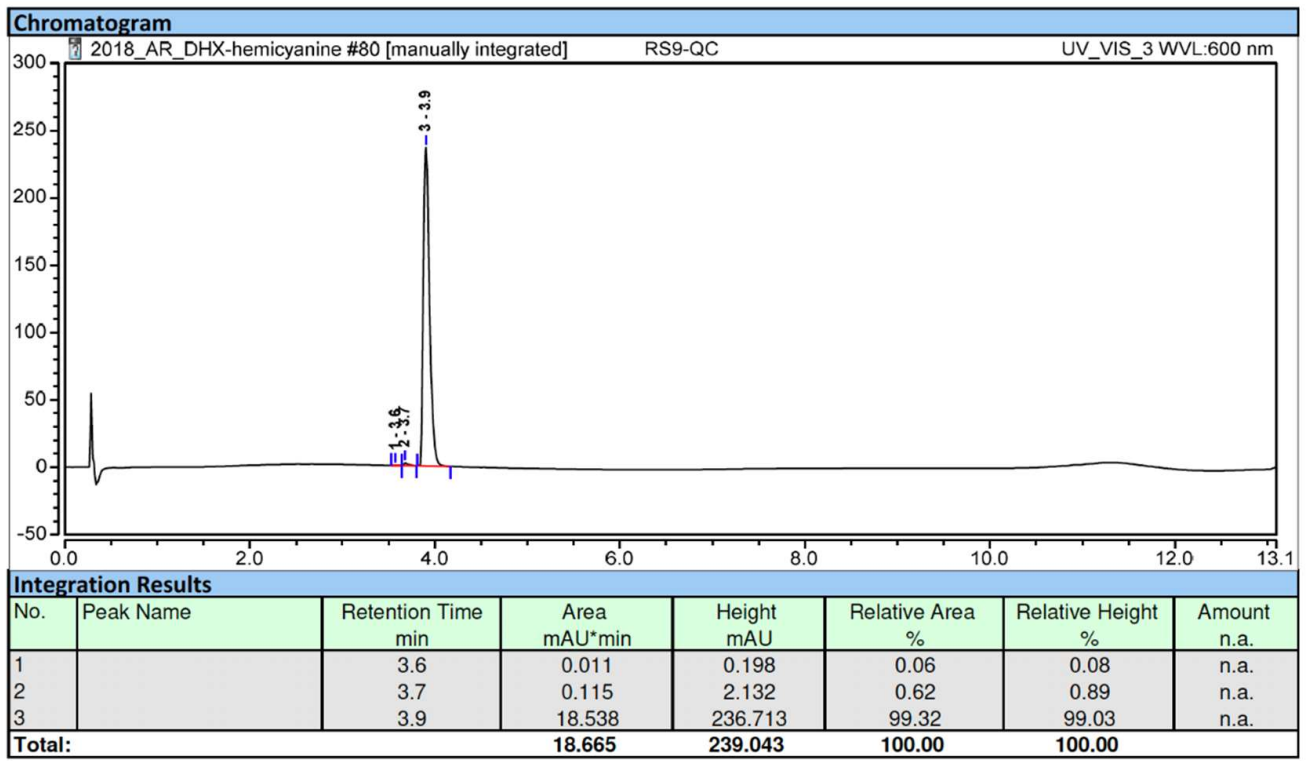

$R P-H P L C$ elution profile of $\mathbf{2 d}$ (system A, detection at $600 \mathrm{~nm}$ )

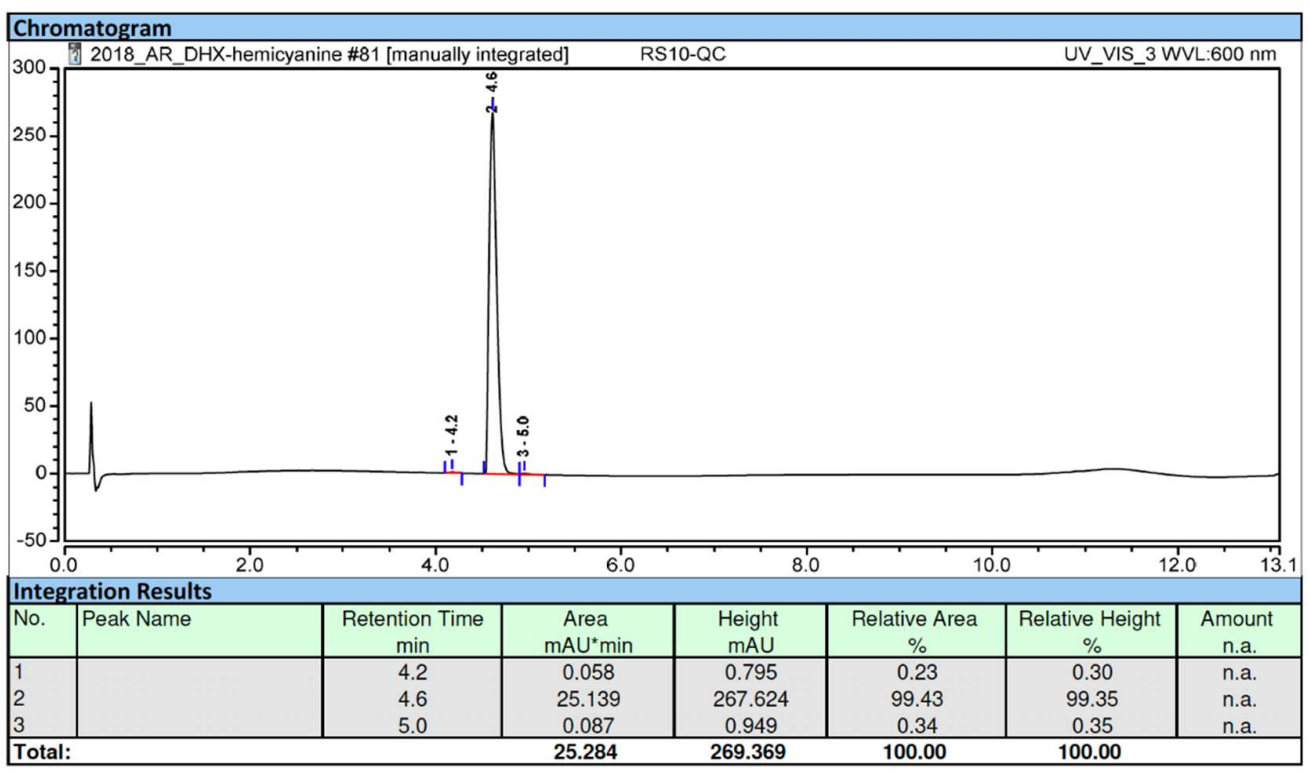


$R P-H P L C$ elution profile of $2 \boldsymbol{e}$ (system A, detection at $600 \mathrm{~nm}$ )

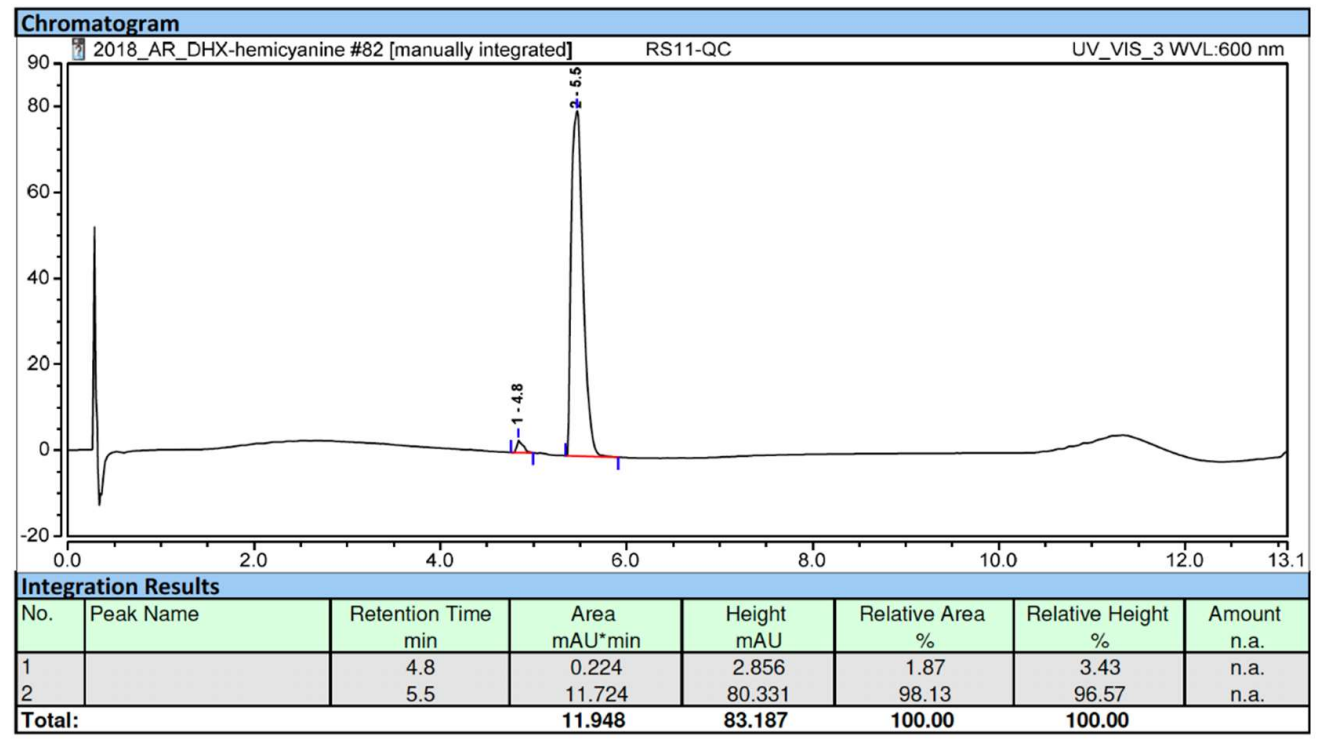

RP-HPLC elution profile of $\mathbf{2 g}$ (system A, detection at $600 \mathrm{~nm}$ )

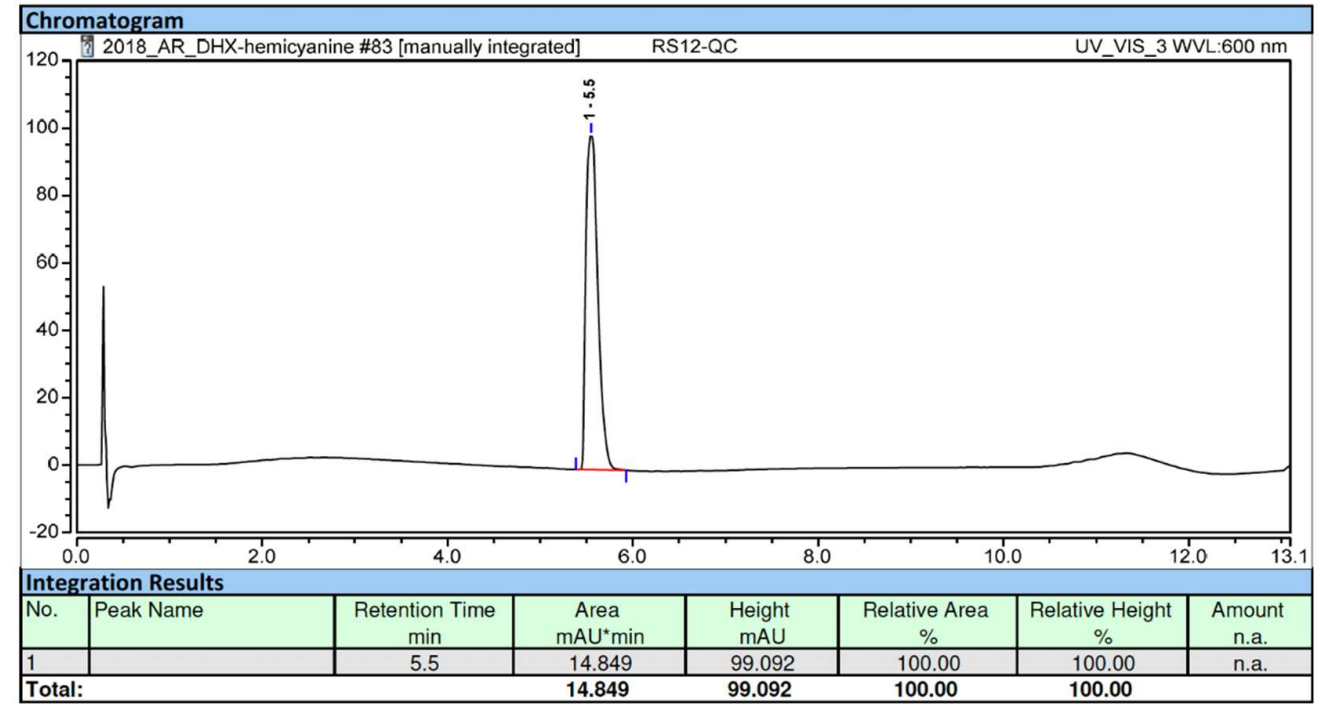


RP-HPLC elution profile of $\mathbf{2 a}($ system A, detection at $600 \mathrm{~nm})$

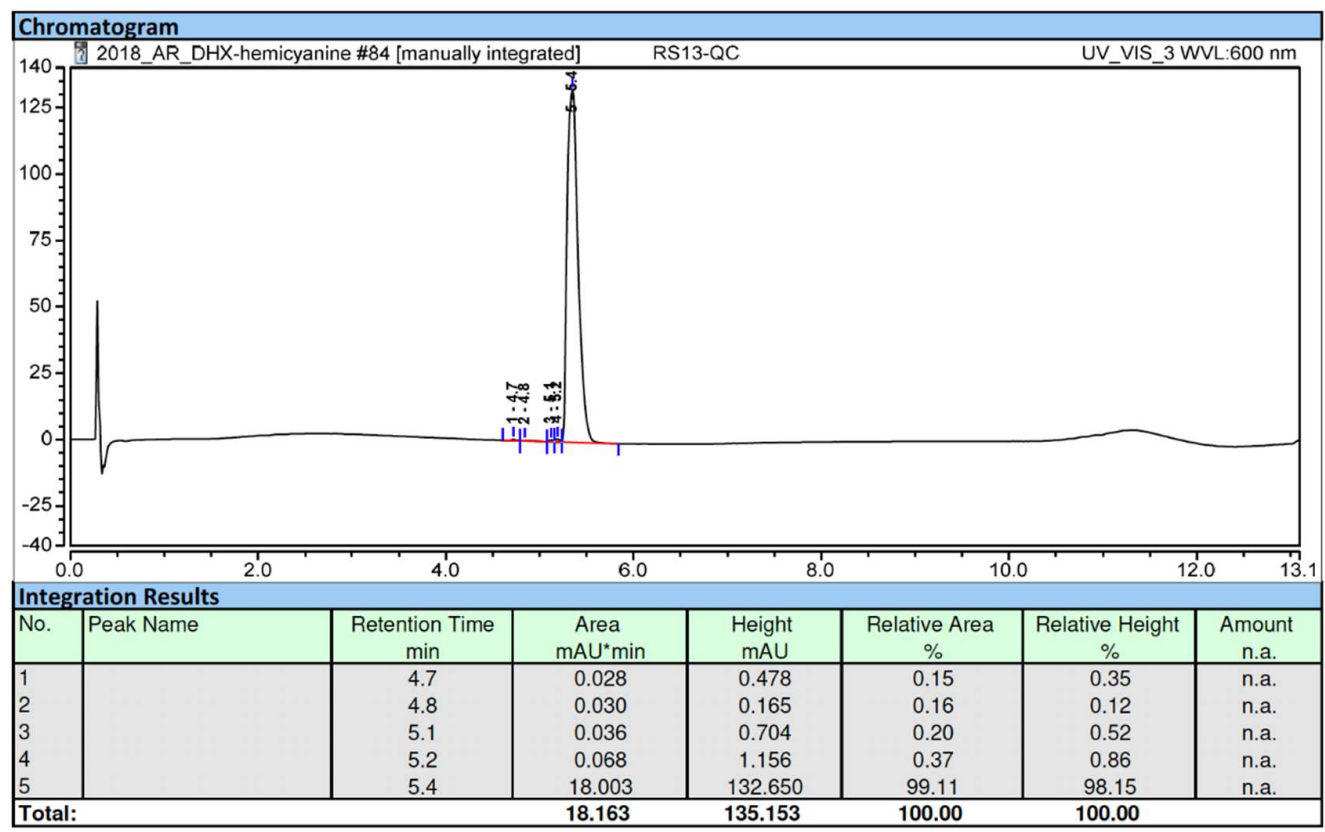

RP-HPLC elution profile of $\mathbf{2} \boldsymbol{k}$ (system A, detection at $600 \mathrm{~nm}$ )

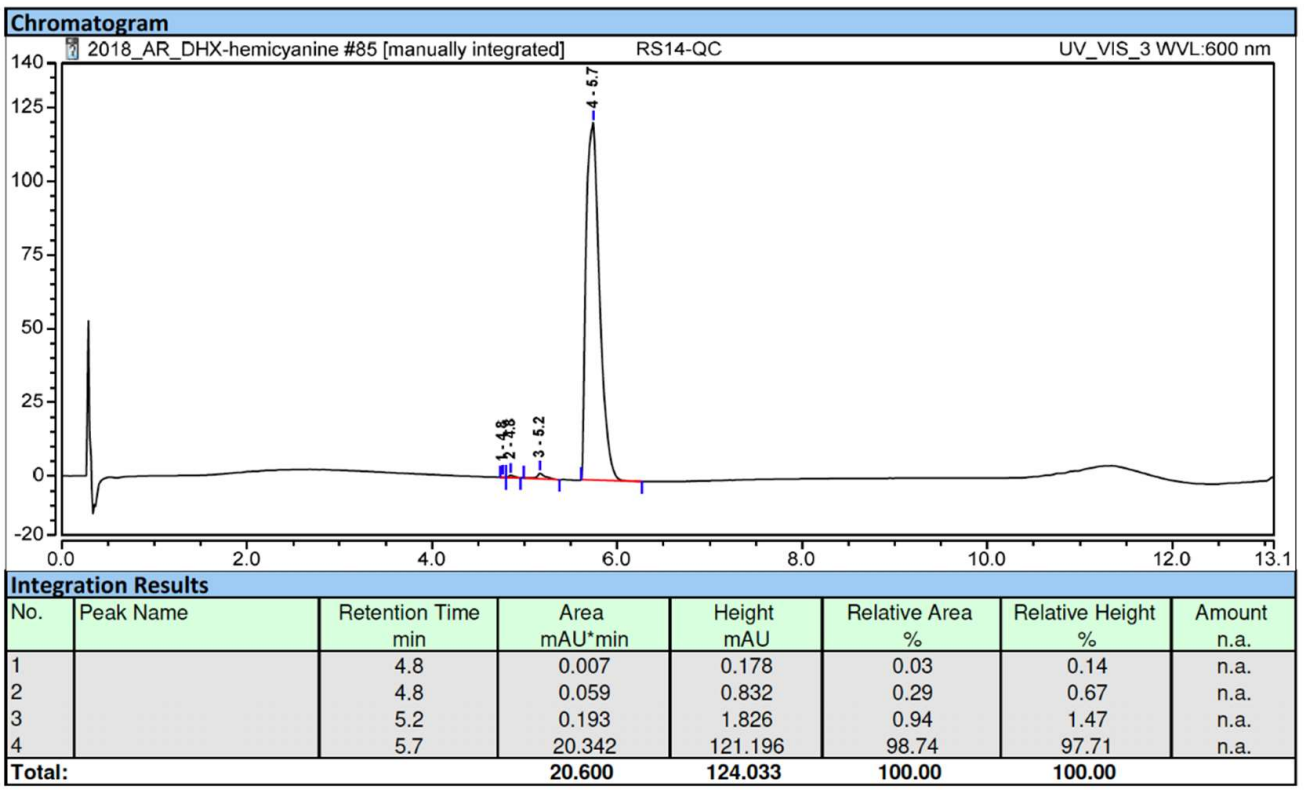


$R P-H P L C$ elution profile of $\mathbf{2 b}$ (system A, detection at $600 \mathrm{~nm}$ )

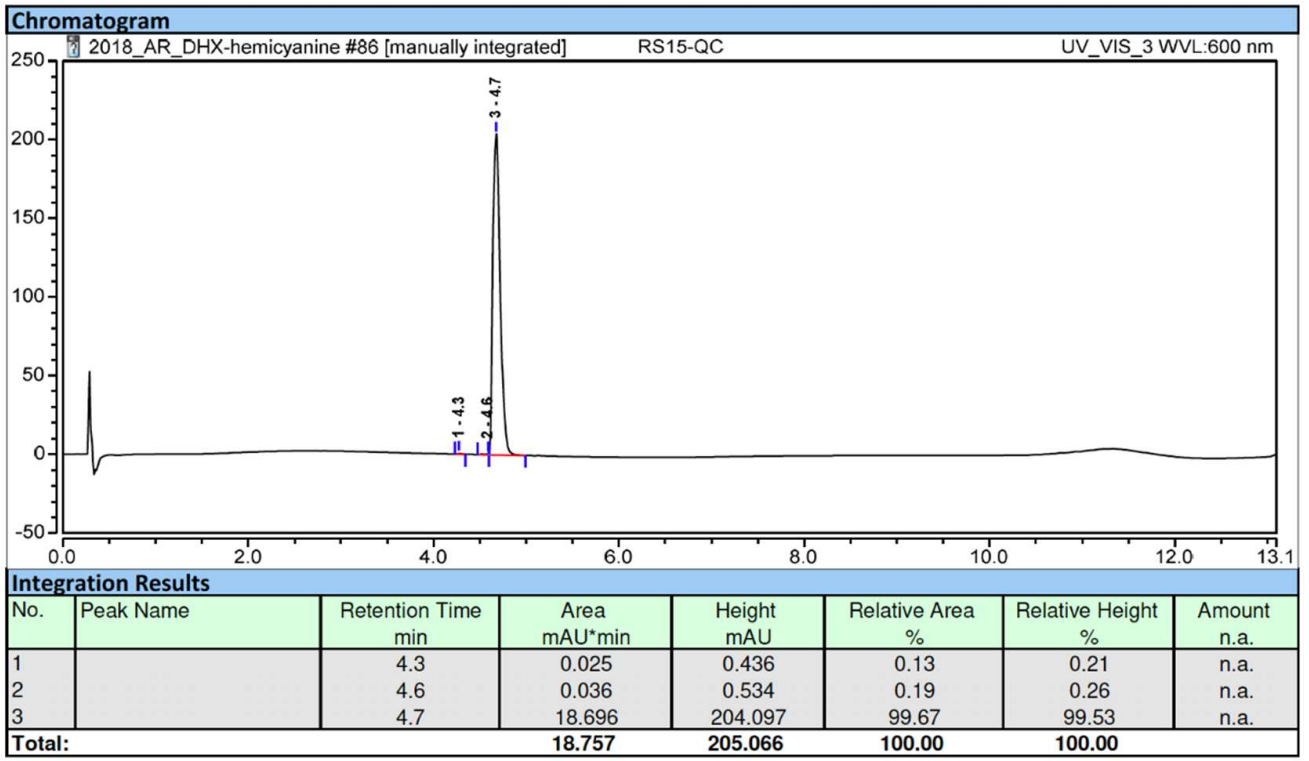

RP-HPLC elution profile of $2 \boldsymbol{p}$ (system A, detection at $600 \mathrm{~nm}$ )

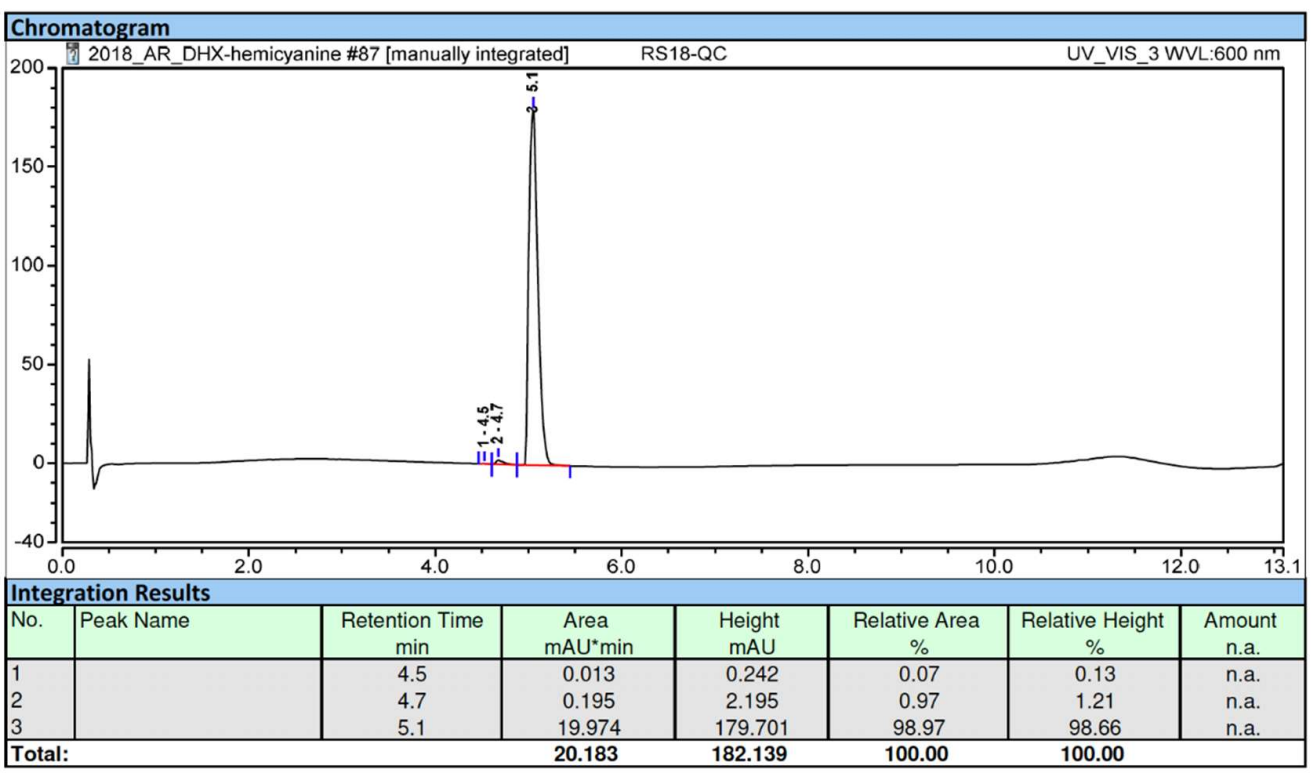


$R P-H P L C$ elution profile of $\mathbf{2 h}$ (system A, detection at $600 \mathrm{~nm}$ )

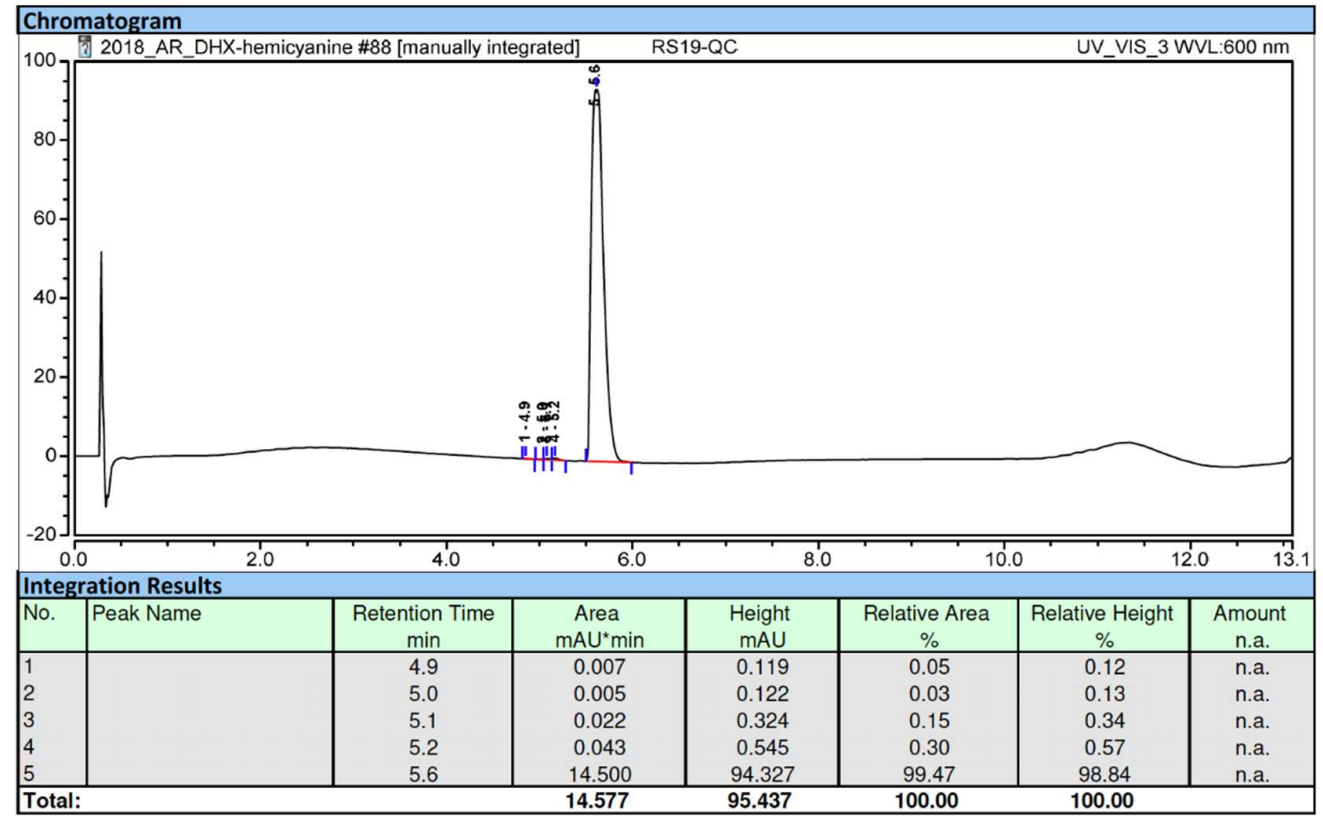

RP-HPLC elution profile of $2 \mathrm{c}$ (system A, detection at $600 \mathrm{~nm}$ )

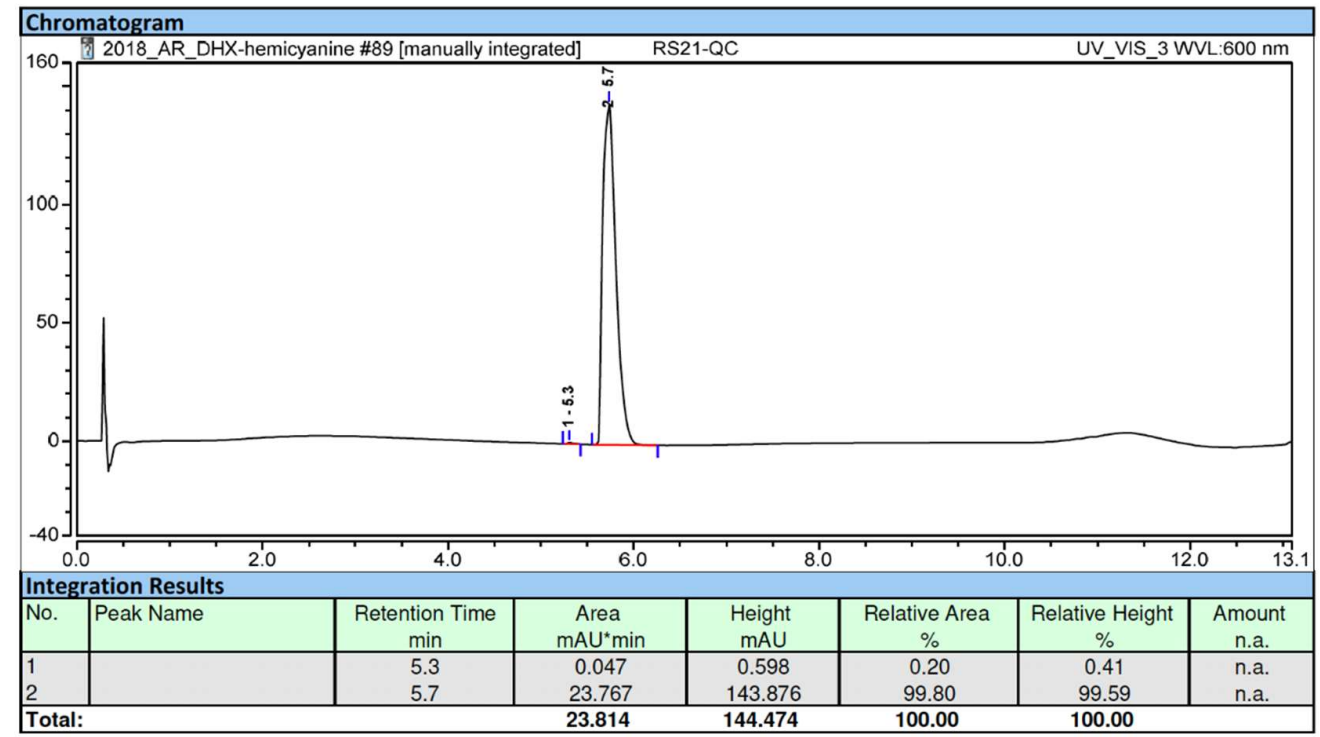


$R P-H P L C$ elution profile of $2 \boldsymbol{j}$ (system A, detection at $600 \mathrm{~nm}$ )

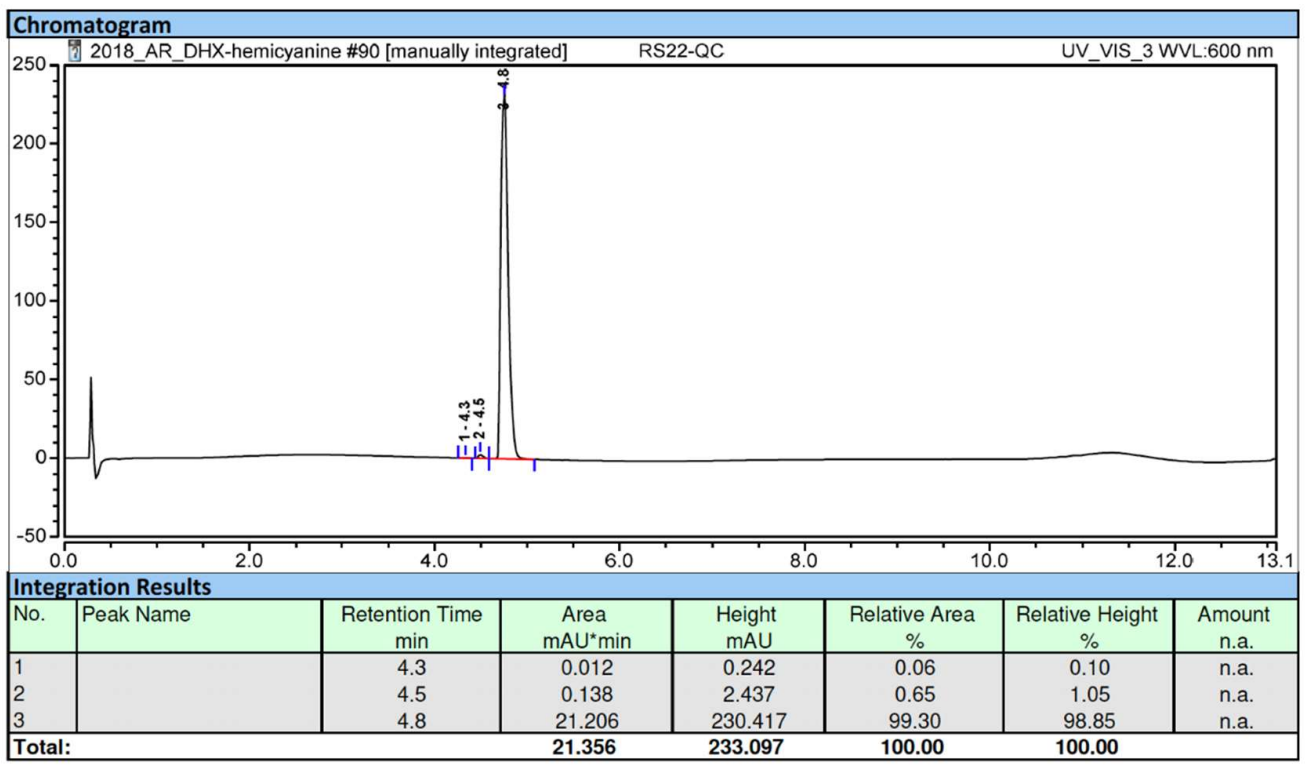

$R P$-HPLC elution profile of $2 \boldsymbol{f}$ (system A, detection at $600 \mathrm{~nm}$ )

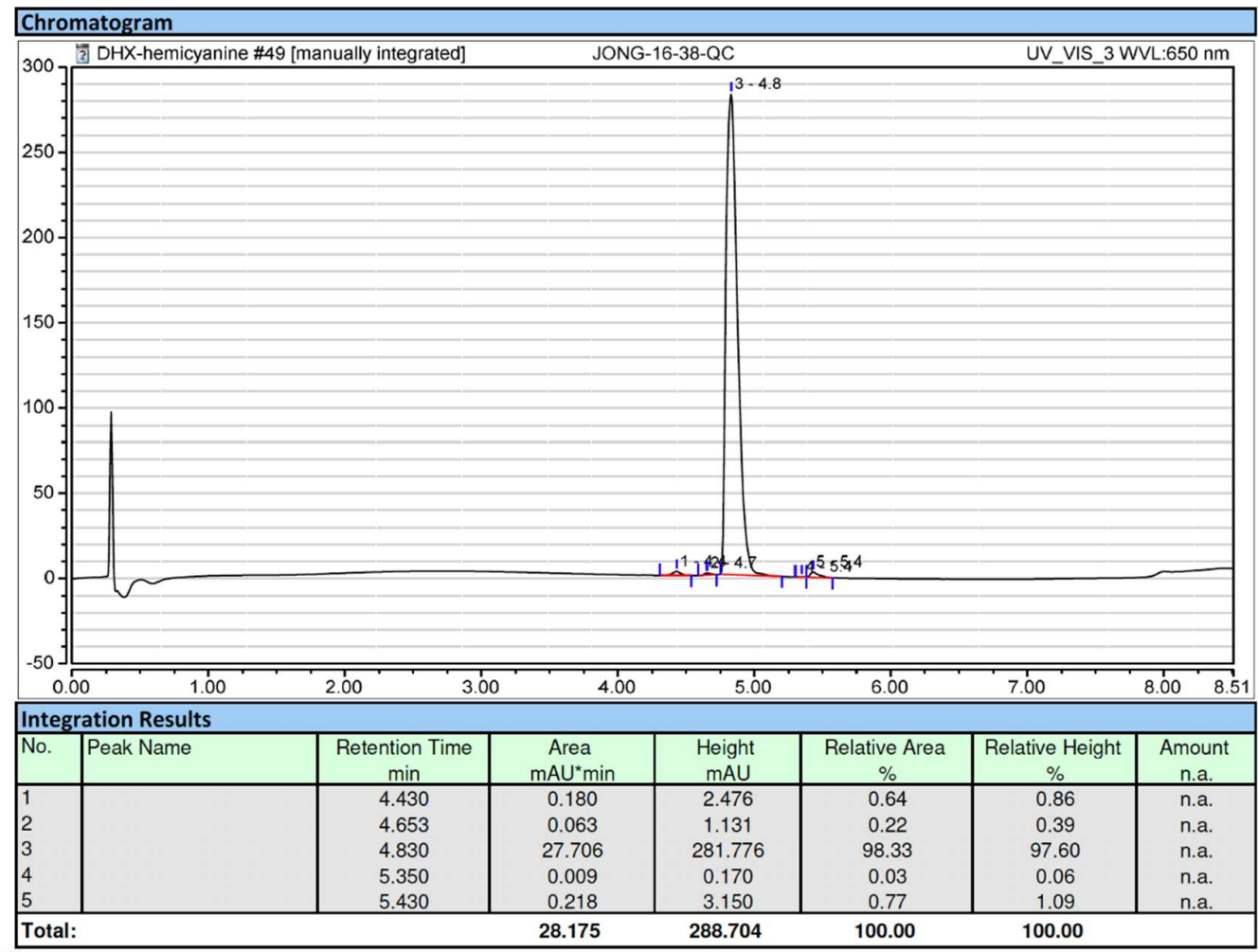




\section{UV-visible absorption spectra of compound 1 and non-fluorescent $5^{\prime}, 7^{\prime}$ - difluoro-DHX-hemicyanine fused dyes}

Compound 1

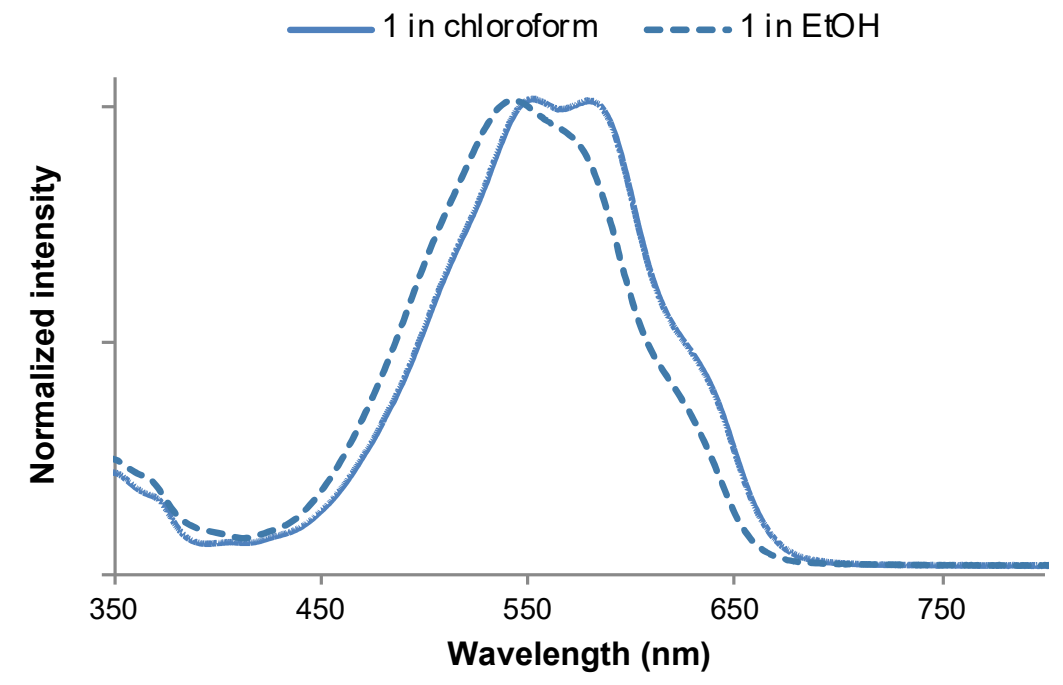

Compound $2 q$

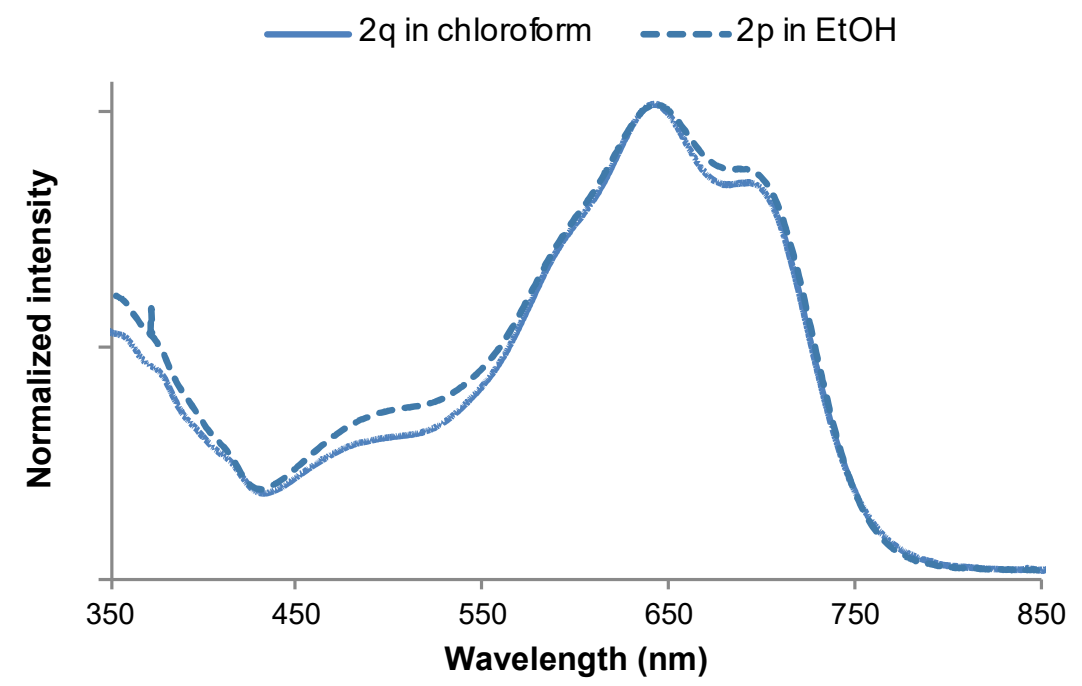


Compound $2 \mathrm{~s}$

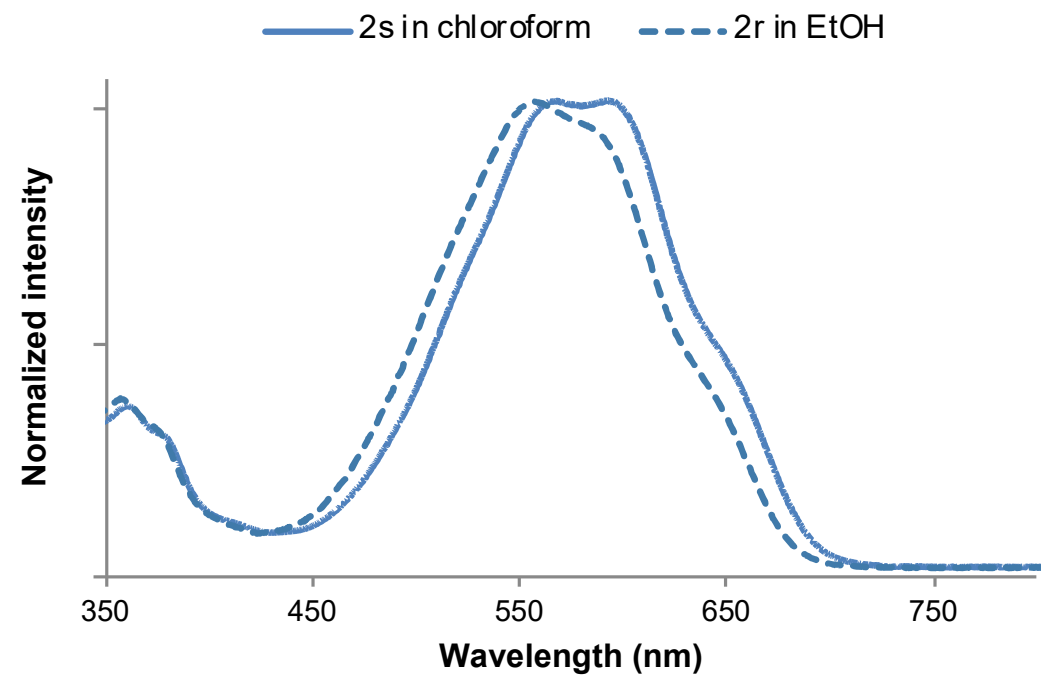

Compound $2 v$

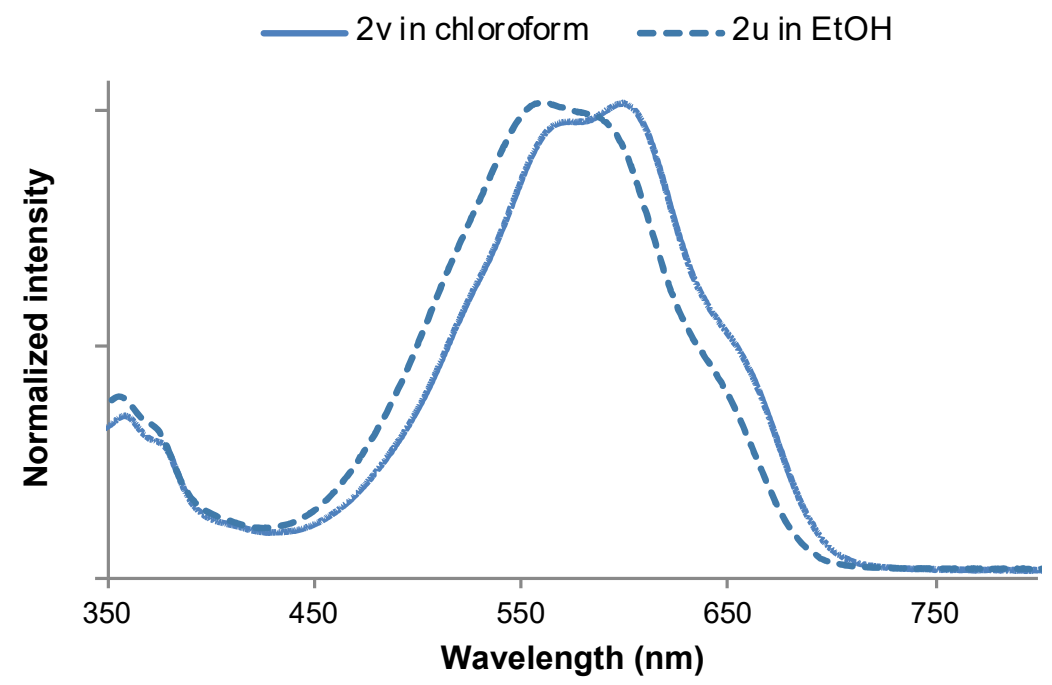

\section{Absorption, excitation and emission spectra of selected 5',7'-difluoro-6'- amino-DHX-hemicyanine fused dyes}

Please note: All emission spectra are corrected until $850 \mathrm{~nm}$, which explains the artefact observed at this wavelength. $U V$-vis spectra recorded in the range $10^{-6}-10^{-5} \mathrm{M}$ for determination of molar extinction coefficients. Fluorescence Ex/Em spectra recorded in the range $10^{-7}-10^{-6} \mathrm{M}$ for $Q Y$ determinations. 


\section{Compound $2 a$}

EtOH, $25^{\circ} \mathrm{C}$, excitation at $615 \mathrm{~nm}$, emission at $760 \mathrm{~nm}$.

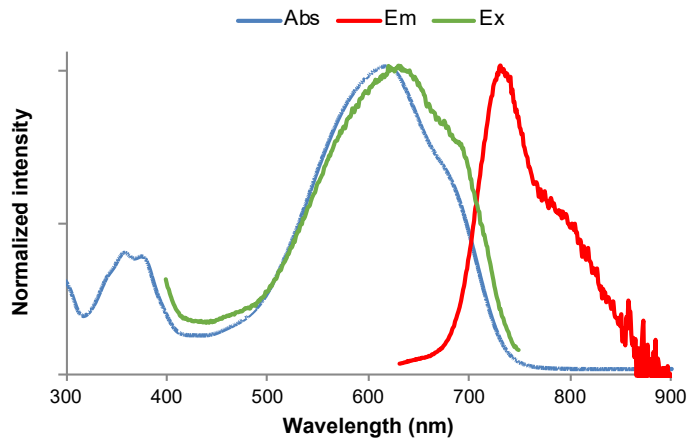

\section{Compound $2 \boldsymbol{d}$}

$\mathrm{CHCl}_{3}$ (left) \& $\mathrm{EtOH}$ (right), $25^{\circ} \mathrm{C}$, excitation at $615 \mathrm{~nm}$, emission at $760 \mathrm{~nm}$.
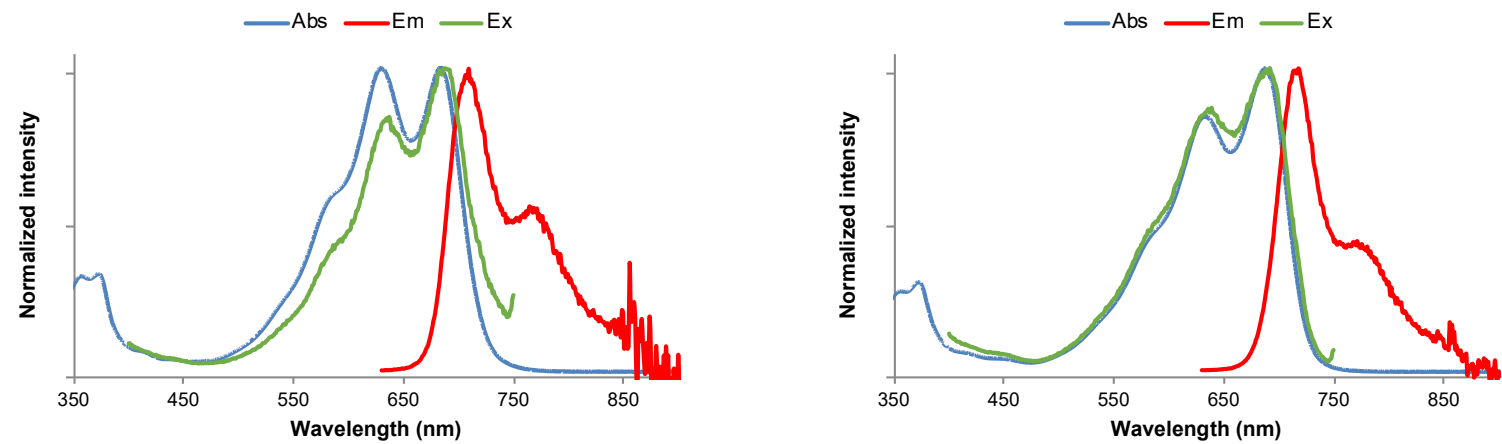

\section{Compound $2 \boldsymbol{e}$}

EtOH, $25^{\circ} \mathrm{C}$, excitation at $615 \mathrm{~nm}$, emission at $760 \mathrm{~nm}$.

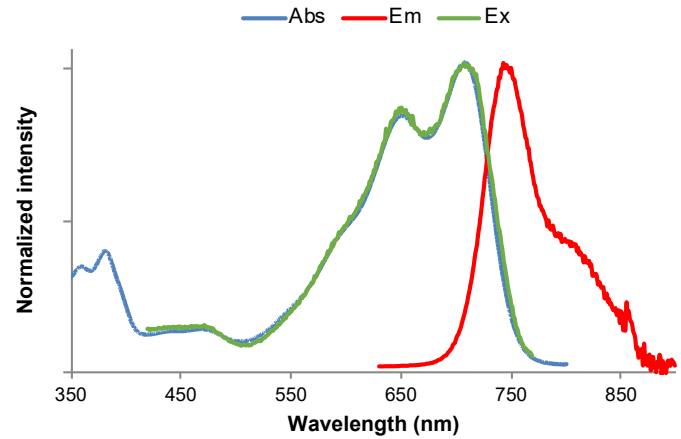




\section{Compound $2 g$}

$\mathrm{CHCl}_{3}$ (top left), $\mathrm{EtOH}$ (top right) \& $\mathrm{PBS}+5 \% \mathrm{BSA}$ (bottom), $25^{\circ} \mathrm{C}$, excitation at $615 \mathrm{~nm}$, emission at $800 \mathrm{~nm}$ (for Ex spectra in $\mathrm{CHCl}_{3} \& \mathrm{EtOH}$ ), emission at $760 \mathrm{~nm}$ (for Ex spectrum in $\mathrm{PBS}+5 \% \mathrm{BSA}$ ).
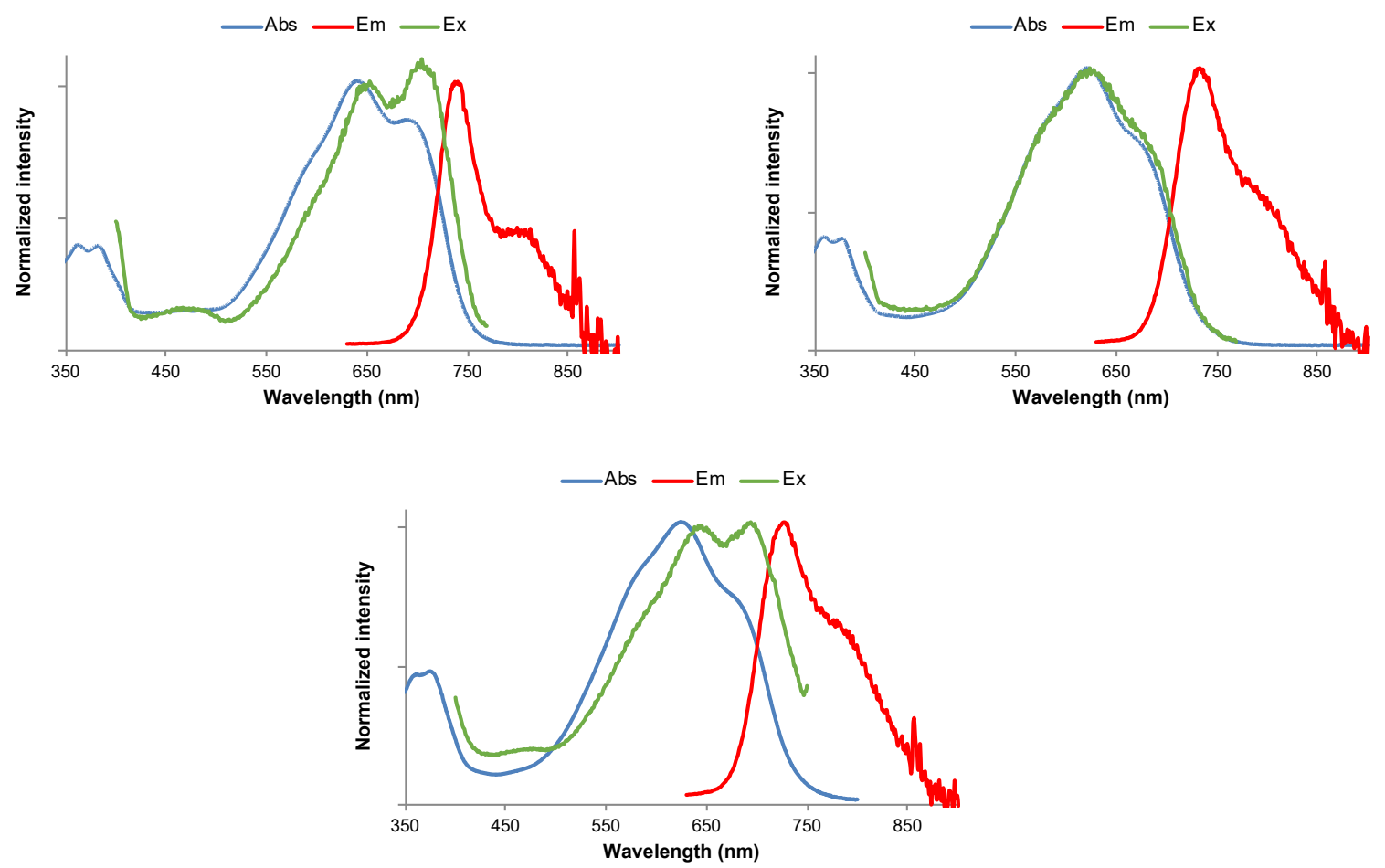

\section{Compound $2 \boldsymbol{k}$}

$\mathrm{CHCl}_{3}$ (top left), $\mathrm{EtOH}$ (top right) \& $\mathrm{PBS}+5 \% \mathrm{BSA}$ (bottom), $25^{\circ} \mathrm{C}$, excitation at $615 \mathrm{~nm}$, emission at $800 \mathrm{~nm}$ (for Ex spectra in $\mathrm{CHCl}_{3} \& \mathrm{EtOH}$ ), emission at $760 \mathrm{~nm}$ (for Ex spectrum in $\mathrm{PBS}+5 \% \mathrm{BSA}$ ).
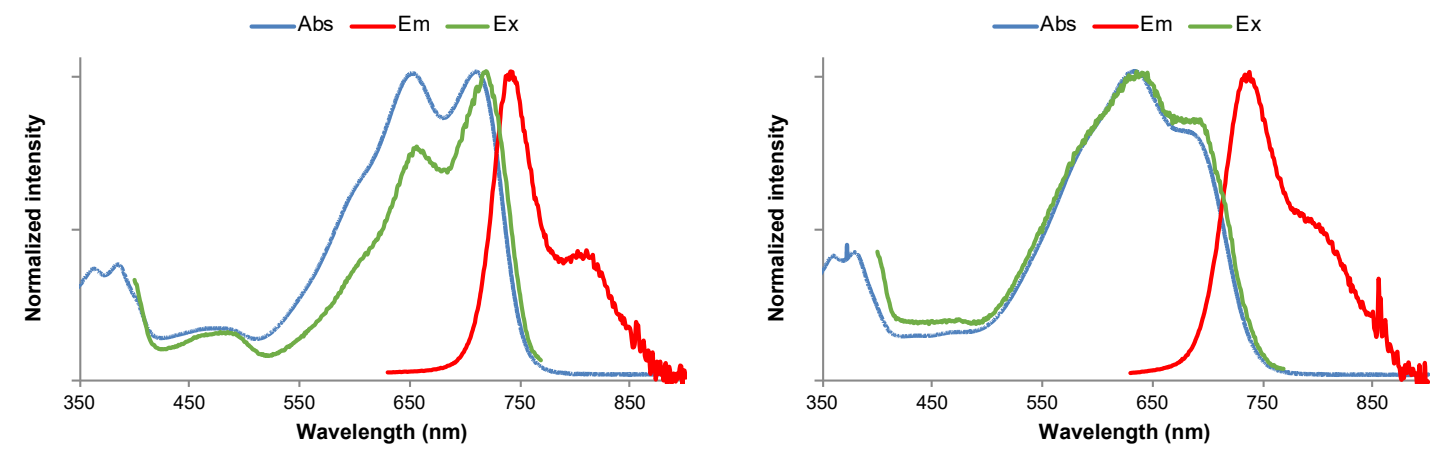


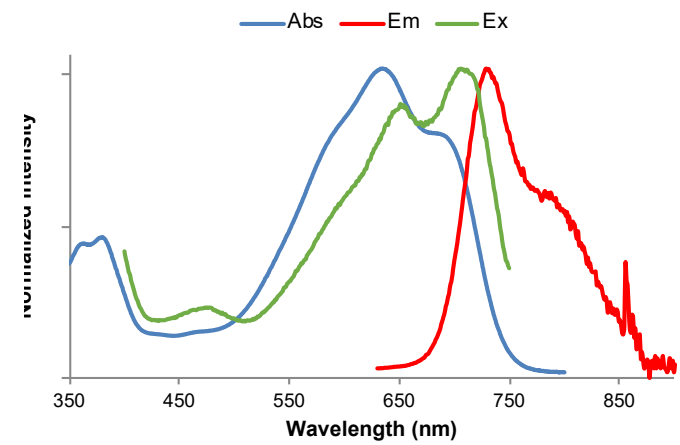

\section{Compound 8}

$\mathrm{CHCl}_{3}$ (left) \& $\mathrm{EtOH}$ (right), $25{ }^{\circ} \mathrm{C}$, excitation at $615 \mathrm{~nm}$, emission at $760 \mathrm{~nm}$.
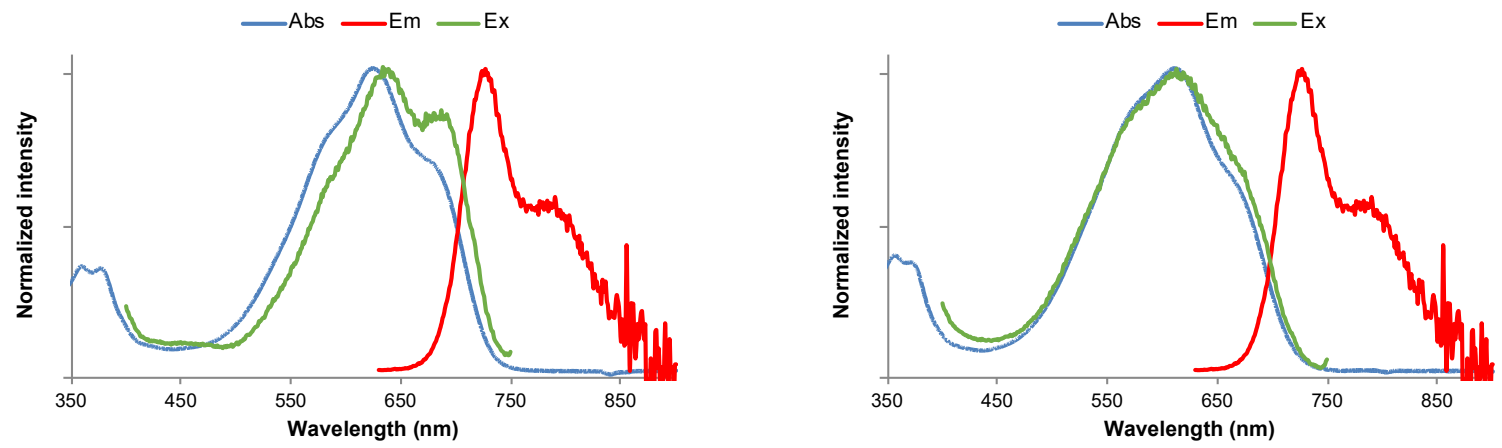